\title{
Größenselektive Untersuchungen zur Kristallisation und Struktur von Wasserclustern
}

\author{
Dissertation \\ zur Erlangung des mathematisch-naturwissenschaftlichen Doktorgrades \\ "Doctor rerum naturalium" \\ an der Georg-August-Universität zu Göttingen \\ im Promotionsprogramm Chemie \\ der Georg-August-University School of Science (GAUSS)
}

vorgelegt von

Christoph Czeslaw Pradzynski

aus Wejherowo / Polen

Göttingen, 2014 
Betreuungsausschuss:

PD Dr. Thomas Zeuch, Institut für Physikalische Chemie, Georg-August-Universität Göttingen

Prof. Dr. Martin A. Suhm, Institut für Physikalische Chemie,

Georg-August-Universität Göttingen

Mitglieder der Prüfungskommission:

Referent: PD Dr. Thomas Zeuch, Institut für Physikalische Chemie,

Georg-August-Universität Göttingen

Korreferent: Prof. Dr. Martin A. Suhm, Institut für Physikalische Chemie,

Georg-August-Universität Göttingen

weitere Mitglieder der Prüfungskommission:

Jun.-Prof. Dr. Ricardo Mata, Institut für Physikalische Chemie,

Georg-August-Universität Göttingen

Prof. Dr. Burkhard Geil, Institut für Physikalische Chemie,

Georg-August-Universität Göttingen

Prof. Dr. Karlheinz Hoyermann, Institut für Physikalische Chemie,

Georg-August-Universität Göttingen

Prof. Dr. Jörg Schröder, Institut für Physikalische Chemie,

Georg-August-Universität Göttingen

Tag der mündlichen Prüfung: 06.02.2015 
Während der Anfertigung dieser Arbeit erhielt ich sehr viel Unterstützung, für die ich mich bedanken möchte. Mein besonderer Dank gilt PD Dr. Thomas Zeuch für die Betreuung der Arbeit, die Diskussion der Ergebnisse und seinen Enthusiasmus bei der Bewältigung der auftretenen Schwierigkeiten. Bei Prof. Dr. Martin Suhm möchte ich mich herzlich für die Aufnahme in die Arbeitsgruppe, die Übernahme des Korreferats sowie die fortwährende Unterstützung unserer Untergruppe bedanken. Dank gilt Prof. Dr. Udo Buck, ohne den es diese Apparatur nicht gäbe und der uns mit neuen Denkanstößen hilft, unseren Beitrag zu aktuellen Forschungsfragen zu leisten.

Bei der Bewältigung des Messalltags erhielt ich Unterstützung durch Richard Forck, Sabine Wolff, Luisa Huchting, Jozef Lengyel, Florian Zurheide und Christoph Dierking. Mein Dank gilt auch den übrigen Mitgliedern der Suhmgruppe für ihre Hilfsbereitschaft und die gute Arbeitsatmosphäre.

Leider blieb mir ein Umzug meiner Apparatur nicht erspart, den ich ohne die Unterstützung der Mitarbeitern der Elektronik- und Mechanikwerkstätten unter der Leitung der Herren Knorr und Meyer nicht ohne weiteres bewältigt hätte. In diesem Zusammenhang gilt ganz großer Dank Herrn Noack, ohne den unsere Apparatur weder über Kühlwasser noch Strom verfügen würde. Der Glaswerkstatt unter der Leitung von Frau von Roden sowie der Pumpenwerkstatt von Herrn Wichmann danke ich für ihre Beratung und ihren Service. Ohne Herrn Bürsing, der sich die Zeit genommen hat, unseren Excimer-Lasers bis zum Schluss am Leben zu erhalten, wäre diese Arbeit nur halb so lang und ohne die Lektoren Florian Zurheide, Niklas Grünwaldt und Christoph Dierking auch nur halb so verständlich.

Finanziell erhielt ich Unterstützung durch die Deutsche Forschungsgemeinschaft (GRK 782, grant ZE 890-1-1/2) sowie durch die Georg-August-Universität Göttingen, die uns ermöglichte ein neues UV/Vis-Lasersystem anzuschaffen.

Für ihre Aufmunterung und Geduld danke ich meiner Frau Amelie und meinem Sohn Joshua. 



\section{Inhaltsverzeichnis}

$\begin{array}{ll}\text { 1. Einleitung } & 1\end{array}$

2. Grundlagen $\mathbf{5}$

2.1. Erzeugung eines Clusterstrahls . . . . . . . . . . . . . . 5

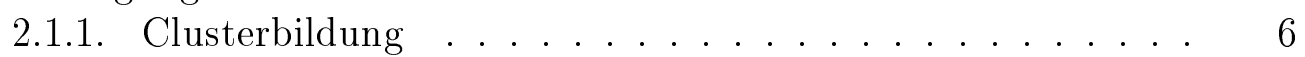

2.2. Wechselwirkungen von Molekülen . . . . . . . . . . . . 9

2.2.1. Strukturen neutraler Wassercluster . . . . . . . . . 10

2.2.1.1. Aggregatzustände. . . . . . . . . . . . . . . 13

2.3. Massenspektrometrie .................... 15

2.3.1. Ionisation . . . . . . . . . . . . . . . 16

2.3.1.1. Photoionisation .............. 18

2.3.1.2. IR-anregungsmodulierte, durch chemische Vorionisation verstärkte Photoionisationsspektroskopie 19

2.3.2. Flugzeitmassentrennung . . . . . . . . . . . . . . . 22

2.4. Quantenchemische Rechnungen .............. 24

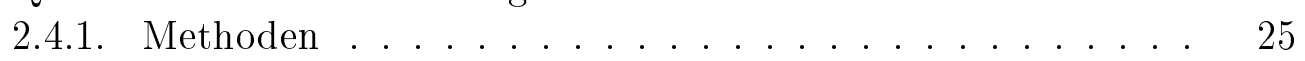

2.4.2. Basissätze . . . . . . . . . . . . . 26

2.4.3. Modellpotentiale ................ 26

\begin{tabular}{|l} 
3. Versuchsaufbau und experimentelle Methoden 29
\end{tabular}

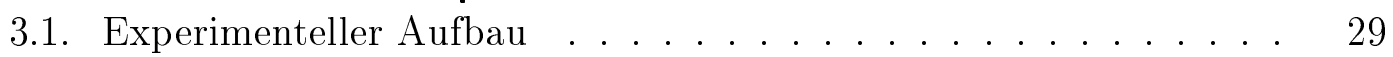

3.1.1. Expansionskammer . . . . . . . . . . . . . . . . . . . 31

3.1.2. Zwischenkammer . . . . . . . . . . . . . . . . . . . . 32

3.1.3. Detektionskammer .................. 35

3.1.3.1. Ionenquelle mit zweistufigem Beschleunigungsfeld 35

3.1.3.2. Reflektron . . . . . . . . . . . . . . 38

3.1.3.3. Detektor. . . . . . . . . . . . . 39

3.1.4. Vakuumsysteme . . . . . . . . . . . . . . . . 41

3.2. Lasersysteme . . . . . . . . . . . . . . . . . . . 42

3.2.1. $\mathrm{UV} /$ Vis-Lasersystem . . . . . . . . . . . . . . 43

3.2.1.1. Excimerlaser gepumptes UV/Vis-Lasersystem . . 43

3.2.1.2. Nd:YAG-Pumplaser Powerlite 9010 . . . . . . . 45

3.2.1.3. Panther EX OPO . . . . . . . . . . . . 50

3.2.1.4. Farbstofflaser Sirah Cobra-Stretch . . . . . . 53 
3.2.2. IR-Lasersystem . . . . . . . . . . . . . 57

3.2.2.1. Nd:YAG-Pumplaser Powerlite 8000 . . . . . . 57

3.2.2.2. LaserVision OPO/OPA . . . . . . . . . . . . 57

3.3. Erzeugung von Messdaten . . . . . . . . . . . . 60

3.3.1. Synchronisation des Massenspektrometers . . . . . . . . 61

3.3.2. Synchronisation der Lasersysteme . . . . . . . . . . . 61

3.3.3. Datenverarbeitung . . . . . . . . . . . . 63

\begin{tabular}{|l|l}
\hline 4. Vergleich mit weiteren experimentellen Methoden & 67
\end{tabular}

4.1. Phenoldotierte Wassercluster. . . . . . . . . . . . . . 67

4.1.1. Experimentelle Methode . . . . . . . . . . . . . 68

4.1.2. Vergleich der IR-Spektren von phenol- und natriumdotierten Clustern im Größenbereich $n=20-50$. . . . . . . . . 69

4.2. Ionische Wassercluster . . . . . . . . . . . . . . 74

4.2.1. Experimentelle Methode . . . . . . . . . . . . 76

4.2.2. Vergleich der IR-Spektren von kationischen und neutralen natriumdotierten Wasserclustern der Größe $n=250 \quad \ldots \quad . \quad 77$

4.3. Direkte Absorption großer Partikel . . . . . . . . . . . . . . 79

4.3.1. $\quad$ FTIR-Spektroskopie an Hüllstromzellen. . . . . . . . . . . 79

4.3.2. $\quad$ Vergleich der IR-Spektren natriumdotierter Cluster mit IRAbsorptionsspektren von Wasseraggregaten. . . . . . . 80

$\begin{array}{ll}\text { 5. Kristallisation von Wasserclustern } & \mathbf{8 7}\end{array}$

5.1. Simulation größenselektiver IR-Spektren . . . . . . . . . . . . . 88

5.2. Experimentelle Spektren . . . . . . . . . . . . . . . . . 91

5.2.1. Kristallisation bei Helium als Trägergas. . . . . . . . . . . 92

$5.2 .2 . \quad$ Kristallisation bei Neon als Trägergas . . . . . . . . . . . . . 97

5.2.3. Vergleich der unterschiedlichen Expansionen . . . . . . . . 100

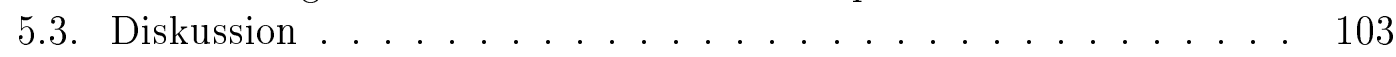

6. Strukturuntersuchung einzelner Clustergrößen 109

6.1. Clustergrößse $n=20$. . . . . . . . . . . . . . . . . 110

6.1.1. Quantenchemische Rechnungen ... . . . . . . . . 110

6.1.2. $\quad$ Experimentelle Daten. . . . . . . . . . . . . . . . . . 113

6.1.3. Vergleich der quantenchemischen und experimentellen Daten 115

6.2. Clustergröß $n=25 \ldots \ldots \ldots \ldots \ldots \ldots$. . . . . . . . . . . . . . . . . . . . . . . . . 120

6.3. Clustergröß $n=32$. . . . . . . . . . . . . . . . . . . . . . . 124

6.4. Zusammenfassung und Zwischenfazit . . . . . . . . . . . . 126

\begin{tabular}{ll}
\hline 7. Zusammenfassung und Ausblick & 131
\end{tabular}

7.1. Zusammenfassung . . . . . . . . . . . . . . 131

7.2. Ausblick . . . . . . . . . . . . . . . . . . 133 
A. Anhang I

A.1. Verwendete Chemikalien ................ III

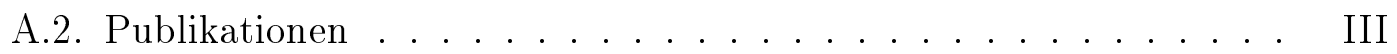

A.3. Auflistung der Expansionsparameter der verwendeten Messreihen. VI

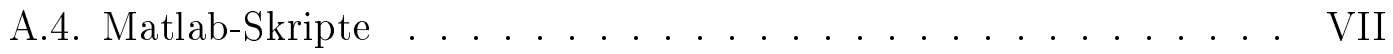

A.4.1. Nach Clustergrößen aufgelöste Auswertung . . . . . . . . . VII

A.4.2. Auswertung nach Größenbereichen .......... . XI

\begin{tabular}{ll}
\hline Literaturverzeichnis & XVII
\end{tabular} 



\section{Einleitung}

Wasser ist eine der am weitesten verbreiteten Substanzen auf der Erde. Ohne den Wasserkreislauf bestehend aus Verdunstung, Kondensation und Niederschlag wäre das Leben auf der Erde nicht möglich. Diese Prozesse sind stark abhängig von der Nukleation und dem Wachstum wasserhaltiger Partikel in der Erdatmosphäre, sowie von deren Oberflächenreaktionen $[1-4]$.

Die besonderen physikalischen Eigenschaften des Wassers, wie zum Beispiel die Dichteanomalie, die schon vor über 300 Jahren dokumentiert wurde [5], sind auf unterschiedliche Wasserstoffbrückentopologien in flüssiger und fester Phase zurückzuführen. Die Wassermoleküle in Eis, wie es unter atmosphärischen Bedingungen vorliegt, bilden ein symmetrisches, dreidimensionales Netzwerk aus sechsgliedrigen Ringen aus. Jedes Molekül ist hierbei vierfach koordiniert. In flüssigem Wasser hingegen treten fünfgliedrige Ringe sowie teilweise dreifach koordinierte Moleküle auf. Das gesamte Netzwerk ist dabei nicht starr, sondern seine Struktur fluktuiert innerhalb von Picosekunden [6,7].

Um diese makroskopischen Effekte besser zu verstehen, hat sich die Clusterspektroskopie als Methode zur mikroskopischen Strukturaufklärung bewährt [8]. Da die Eigenschaften der Cluster stark von ihrer Größe abhängig sind, ist eine vollständige Größenselektivität für ihre Erforschung vonnöten. Zusätzlich wird ein Verfahren benötigt, das sensitiv auf die Struktur der wasserstoffbrückengebundenen Cluster ist. Die IR-Spektroskopie, insbesondere der OH-Streckschwingung, stellt ein solches Verfahren dar, da die Ausbildung der intermolekularen Wasserstoffbrückenbindung zu einer Schwächung der intramolekularen Bindung des Donormoleküls führt und damit einen direkten Effekt auf die Absorption des betreffenden OH-Oszillators hat [9,10]. Es kann dabei eine Rotverschiebung von mehr als $700 \mathrm{~cm}^{-1}$ im Vergleich zum ungebundenen Oszillator stattfinden [11. Bei kleinen Clustern mit bis zu fünf Wassermolekülen kann die Absorption der einzelnen gebundenen Oszillatoren mit Hilfe von FTIR-Spektroskopie gemessen werden, da 
diese in unterschiedlichen Wellenlängenbereichen absorbieren 12 14. Vor Kurzem wurde das Raman-Streu-Spektrum von isolierten Wasserclustern in diesem Größenbereich publiziert [15]. Die Kombination von Raman und IR-Spektroskopie ermöglicht einen Einblick in die Kopplung der gebundenen OH-Oszillatoren, die für den schnellen Energietransfer in Wasser verantwortlich sind, und stellt eine Möglichkeit der Verbesserung der quantenchemischen Modellpotentiale dar 15. Eine weitere Methode zur Untersuchung von isolierten Teilchen in der Gasphase ist die Mikrowellenspektroskopie, welche die reine Rotationsanregung der Moleküle detektiert. Mit einer Weiterentwicklung dieser Methode, bezeichnet als chirped pulse Fourier transform mikrowave (CP-FTMW) Spektroskopie [16], konnte die Isomerenzusammensetzung des Wasserhexamers [17] und -heptamers 18 untersucht werden. Eine Strukturaufklärung ist durch den Vergleich mit quantenchemischen Rechnungen möglich. Die Größenselektion wird mit Hilfe der unterschiedlichen Absorptionseigenschaften der Wasseraggregate gewährleistet.

Eine vollständige Größenselektion für neutrale Spezies, die nicht auf den unterschiedlichen Absorptionseigenschaften der Cluster beruht, kann mit der Streumethode aus der Gruppe von Buck erreicht werden, bei welcher der Clusterstrahl im $90^{\circ}$-Winkel auf einen aus Edelgasatomen bestehenden Atomstrahl trifft. Sind der Streuwinkel und die resultierende Geschwindigkeit bekannt, können unabhängig von der Detektionsmethode Rückschlüsse auf die Clustergröße gezogen werden [19|. Die Erzeugung des IR-Spektrums erfolgt durch IR-Photoneninduzierte Dissoziation (Infrared (multi)photon dissociation, IR(M)PD) [20-22]. Diese indirekte Form der Erzeugung von Schwingungsspektren wird als Wirkungsspektroskopie (action spectroscopy) bezeichnet. Die Streumethode ermöglichte bisher die Strukturaufklärung von Aggregaten mit bis zu 10 Wassermolekülen [9,23, 24,.

Bei der Erforschung größerer Cluster werden aufgrund der kleineren relativen Massenunterschiede an Massenspektrometer gekoppelte Verfahren angewendet. Da eine Separierung der Cluster durch elektrische oder magnetische Felder für ionische Spezies möglich ist, liegt der Fokus dieser Studien meist auf kationischen 25-28] oder anionischen 26, 29, 31 Wasserclustern. In den meisten Arbeiten 25, 27, 31 wird die Clustergröße mit Hilfe eines Massenfilters ausgewählt und dann mittels IR(M)PD untersucht. Jedoch hat hier das für die Gesamtladung der Cluster verantwortliche Ion oder Elektron, abhängig von der verwendeten Präparation, einen großen Einfluss auf die Struktur der Spezies, weshalb nur geringe Aussagen über 
die neutrale Spezies getroffen werden können.

Eine wirkungsspektroskopische Untersuchung neutraler Cluster mittels direkter Ionisation ist nicht möglich, da bereits Elektronenstoßionisation (EI) oder direkte Photoionisation mit kurzwelligem UV-Licht (VUV) im Bereich der Ionisationsenergie von Wasser zu einer massiven Fragmentierung der Cluster führen [32 34]. Eine Möglichkeit die Fragmentierung zu reduzieren stellt die Dotierung mit einem Chromophor wie Phenol dar, die eine Ionisation über resonanzverstärkte Zweiphotonenionisation (resonance enhanced multiphotonen ionisation, REMPI) mit gängigen Lasersystemen ermöglicht $35-37$. Für die Erzeugung eines OHStreckschwingungsspektrums wird der Grundzustand der phenoldotierten Cluster mit Hilfe eines IR-Lasers $50 \mathrm{~ns}$ vor der Photoionisation depopuliert, was zu einer Abnahme des Signals der zugehörigen kationischen Spezies im Massenspektrum führt (resonant ion-dip infrared spectroscopy, RIDIR). Die auftretende Fragmentierung durch den Photoionisationsprozess ermöglicht die Zuordnung von IRSpektren zu den Phenol- $\left(\mathrm{H}_{2} \mathrm{O}\right)_{n+\Delta n}$-Clustern mit einer Unsicherheit von $\Delta n \leq 6$. In dieser Arbeit soll gezeigt werden, dass die Dotierung mit Natrium als Chromophor für die größenselektive Untersuchung der Strukturen von Wasserclustern bis zu einer Clustergröße von $n=600$ verwendet werden kann und dass das angelagerte Natriumatom ab einer Clustergröße von ca. $n=19$ nur noch geringen Einfluss auf das Wasserstoffbrückennetzwerk hat. Der für diese Untersuchungen genutzte Effekt der Signalverstärkung durch IR-Absorption bei schwellennaher Ionisation wurde von Steinbach [38 und Buck [39 beschrieben und bisher für kleine bis mittelgroße $(n<50)$ Wasser- $[9,38,41]$ und Methanolcluster $[40-\sqrt{42}$ angewendet. Die Weiterentwicklung dieser Methode soll ermöglichen, in einer Messung (einem IR-Laserscan) über einen weiten Größenbereich (ca. $n=15-600$ ) sowohl die Strukturinformationen einzelner Clustergrößen zu liefern, als auch die Kristallisation von Wasserclustern größenselektiv zu untersuchen. Bisher ist es auf auf Basis von Schwingungsspektren lediglich möglich, die Absorption einer Größenverteilung, die Eispartikel mit einem mittleren Durchmesser von 4-12 nm enthält, zu charakterisieren 43,44. Das Einsetzen der Kristallisation von Wasserclustern wurde in Elektronenbeugungsexperimenten von Torchet und Mitarbeitern auf einen Größenbereich von $n=200-1000$ abgeschätzt [45], was im messbaren Bereich der hier verwendeten Methode liegt.

Neben der erwähnten Erweiterung der bestehenden Methode auf die Clustergrößen 
$n=15-600$ ist das Ziel dieser Arbeit, die Auswirkung der Expansionsbedingungen durch Variation der Trägergase Helium, Neon und Argon sowie der Stagnationsdrücke auf die Kristallisation der Wassercluster und Isomerenzusammensetzung einzelner Clustergrößen zu untersuchen.

Die vorliegende Arbeit ist wie folgt strukturiert: Kapitel 2 beinhaltet eine Einführung in die Erzeugung der Cluster sowie die größenselektive Detektion. Es wird zusätzlich auf zwischenmolekulare Wechselwirkungen der Wassermoleküle eingegangen, ein Einblick in die Aggregatzustände von Wasser und eine kurze Einführung in quantenmechanische Methoden wird gegeben. In Kapitel 3 soll das bereits in mehreren Publikationen vorgestellte 40,42, 46, 47], vorliegende Experiment einschließlich eines neuen UV/Vis-Lasersystems beschrieben und die IRanregungsmodulierte Photoionisationsmethode (IR-CIPI) vorgestellt werden. In Kapitel 4 wird die Vielseitigkeit dieser Methode durch einen Vergleich mit anderen Experimenten wie der Dotierung mit Phenol [37], der Erzeugung ionischer Cluster [26, 28] oder auch direkter Absorption [43, 44, dargelegt. Die größenselektive Untersuchung der Kristallisation von Wasserclustern ist Bestandteil von Kapitel 5. Das Potential der Methode wird anhand der vollständig größenselektiven Strukturuntersuchung der Cluster $\left(\mathrm{H}_{2} \mathrm{O}\right)_{20},\left(\mathrm{H}_{2} \mathrm{O}\right)_{25},\left(\mathrm{H}_{2} \mathrm{O}\right)_{32}$ auch unter Berücksichtigung mehrerer Isomere in Kapitel 6 aufgezeigt. Außerdem werden erste experimentelle Hinweise auf auffällige Clustergrößen dargelegt. Kapitel 7 beinhaltet eine Zusammenfassung der erhaltenen Ergebnisse sowie einen kurzen Ausblick auf mögliche ergänzende Untersuchungen. 


\section{Grundlagen}

In diesem Kapitel wird eine kurze Einführung in die für das Verständnis der Arbeit nötigen Grundlagen gegeben. Es wird den Fragen nachgegangen, wie Molekülaggregate (Cluster) erzeugt werden, welche Wechselwirkungen zwischen einzelnen Molekülen auftreten, wie sie größenselektiv analysiert werden können und welche Phasenumwandlungen in makroskopischem Wasser und Wasserpartikeln bekannt sind.

\subsection{Erzeugung eines Clusterstrahls}

Die Erzeugung eines Clusterstrahls erfolgt mittels adiabatischer Überschallexpansion. Bei dieser Methode wird eine Substanz in der Gasphase von einem Bereich hoher Teilchendichte durch eine kleine Öffnung in einen Bereich geringer Teilchendichte überführt. Es werden häufig Edelgase (Helium, Neon oder Argon) als Trägergase verwendet, da diese durch ihre fehlenden Vibrations- und Rotationsfreiheitsgerade über Stöße für eine effektivere Temperierung der Cluster sorgen. Ist die Substanz bei Raumtemperatur flüssig, so kann sie erhitzt werden, um eine möglichst hohe Teilchendichte zu erzeugen. Weitere etablierte Möglichkeiten der Erzeugung sind die Gasaggregationsquelle, die Oberflächenquelle sowie die Nanospray-Ionisation. Für weitere Informationen zu den physikalischen Grundlagen der Methoden sei hier auf die Bücher von Haberland und Scoles 48 51 verwiesen.

Charakterisiert werden kann die Expansion über die Knudsenzahl $K$ :

$$
K=\frac{\lambda_{M}}{d}
$$


Hierbei ist $d$ der Durchmesser der Öffnung und $\lambda_{M}$ die mittlere freie Weglänge,für die der folgende Zusammenhang gilt:

$$
\lambda_{M}=\frac{k_{\mathrm{B}} \cdot T}{\sqrt{2} \cdot p \cdot \sigma}
$$

Diese Gleichung enthält die Bolzmannkonstante $k_{\mathrm{B}}$, die Temperatur $T$, den Druck $p$ und den Stoßquerschnitt der Teilchen $\sigma$. Es werden zwei Grenzfälle unterschieden, bei $K>1$ ist die mittlere freie Weglänge größer als der Düsendurchmesser. Es liegt ein effusiver Strahl vor, bei dem die Moleküle eine hohe Winkeldivergenz aufweisen und deren Geschwindigkeitsverteilung der Maxwell-Boltzmann-Statistik entspricht.

Eine Überschallexpansion liegt vor, wenn $K<<1$ ist. Bei dieser Form der Expansion sind die Atome/Moleküle stark gerichtet und es resultiert eine hohe Teilchendichte im Strahl. Die Moleküle werden stark, bei Verwendung eines Trägergases wenige Kelvin, abgekühlt. Die Erzeugung eines Molekularstrahls aus dem Düsenstrahl erfolgt mit Hilfe eines Skimmers, der die Winkeldivergenz des Strahls weiter minimiert. Bei Verwendung einer Düsenplatte mit konischer Öffnung erhält man Cluster mit einer Temperatur von $50-150 \mathrm{~K}$ [11.

\subsubsection{Clusterbildung}

Die Beschreibung der Clusterbildung erfolgt meist über die klassische Nukleationstheorie [48,52, 53]. Die Bildung der Cluster erfolgt dadurch, dass durch die Temperaturabsenkung bei der Expansion der lokale Druck größer wird als der Dampfdruck in der Gasphase, sodass ein Kondensationsprozess einsetzt. Das dazugehörige $p$-T-Diagramm ist in Abbildung 2.1 dargestellt. Das Verhältnis zwischen dem Sättigungsdampfdruck über einer Flüssigkeit $p_{\infty}$ und dem tatsächlichen Dampfdruck $p_{k}$ ist gegeben über $\Phi_{k}=\frac{p_{k}}{p_{\infty}}$. Der Fall $\Phi_{k}=1$ entspricht der Sättigungsdampfdruckkurve beim Phasenübergang flüssig-gasförmig. Die Temperaturund Druckänderungen bei der Expansion verlaufen zunächst entlang der Adiabate. Sei der Anfangspunkt gegeben durch den Druck $p_{0}$ und die Temperatur $T_{0}$ am oberen Ende der Adiabate, dann entsteht durch die Abkühlung während der Expansion ab Punkt A, dem Schnittpunkt zwischen Adiabate und Dampfdruckkurve, eine Übersättigung des Gases. Im Punkt B setzt die Clusterbildung über 


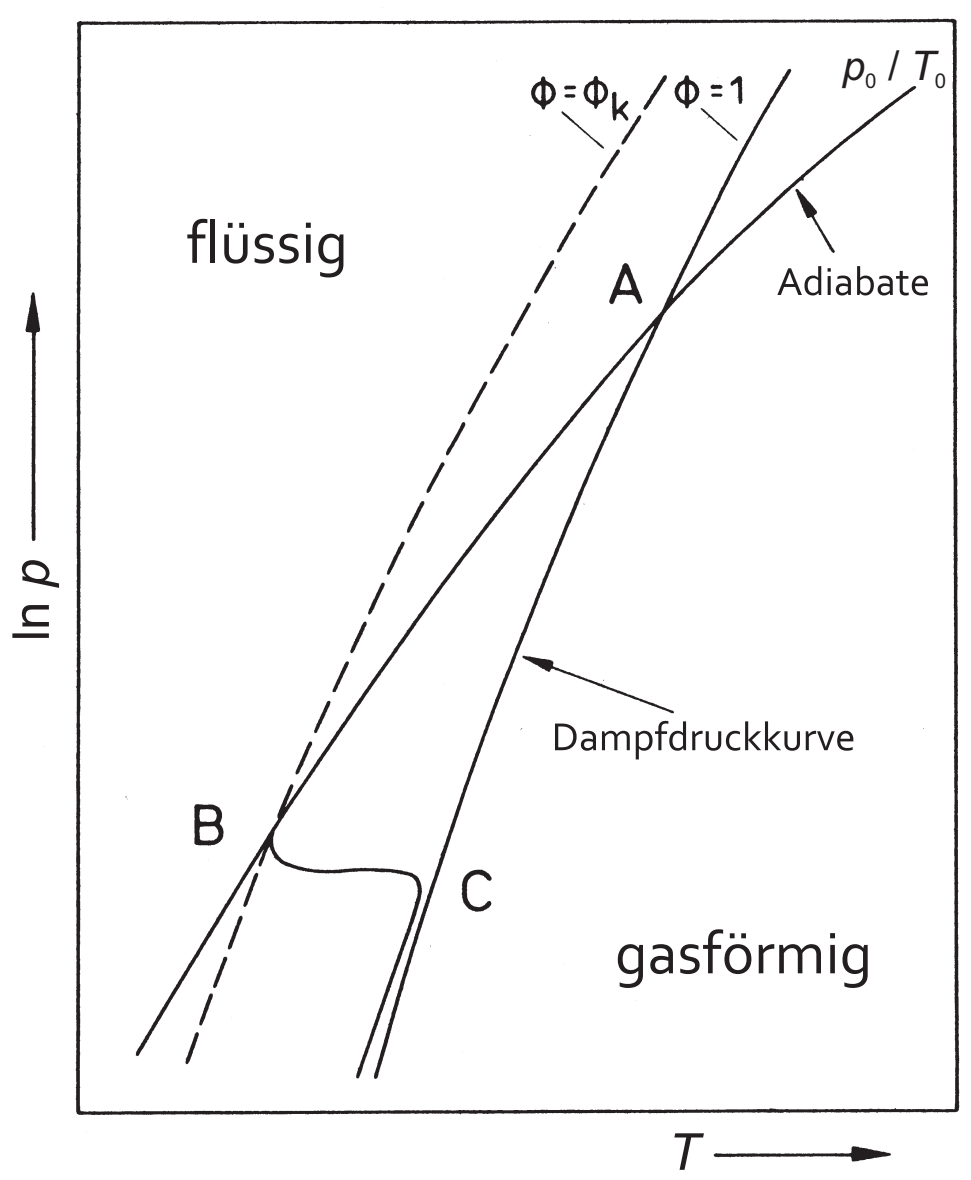

Abbildung 2.1.: Expansions- und Dampfdruckkurve des expandierenden Gases mit dem Ausgangszustand $p_{0}$ und $T_{0}$, dem Übersättigungspunkt $\mathrm{A}$ und dem Kondensationspunkt B, entnommen aus [48].

einen Kondensationsprozess ein. Dieser führt zur Erwärmung der Substanz, sodass die Druck-und Temperaturänderungen nicht mehr dem Kurvenverlauf der Adiabate folgen, sondern im Punkt $\mathrm{C}$ in Richtung der Dampfdruckkurve abknicken. Wenn davon ausgegangen wird, dass am Anfang der Expansion nur Monomere vorliegen, so entstehen Dimere aus Stößen von drei Monomeren, wobei das dritte Teilchen die überschüssige Energie abführt. Dieser Kondensationskeim stößt mit weiteren Monomeren zusammen und bewirkt so ein Wachstum der Cluster. Erst ab größeren Clustergrößen führen Cluster-Cluster-Stöße zu weiterem Wachstum. Dieser Prozess wird als Koagulation bezeichnet. Es ist dabei möglich, Cluster mit mehreren tausend Konstituenten zu erzeugen [54-56]. 
Die durch die Koagulation auftretende Größenverteilung $f(n)$ bei einer Expansion lässt sich über eine logarithmische Normalverteilung modellieren |54,57|:

$$
f(n)=\frac{1}{\sqrt{2 \pi} \cdot \sigma \cdot n} \cdot \exp \left[-\frac{(\ln (n)-\mu)^{2}}{2 \sigma^{2}}\right]
$$

Dabei bezeichnet $n$ die Clustergröße, $\mu$ den Logarithmus des Mittelwerts und $\sigma$ den Logarithmus der geometrischen Standardabweichung. Entstehen Cluster ausschließlich über die Kondensation, so entsteht eine mit der Clustergröße exponentiell abnehmende Größenverteilung. Diese ist abhängig von der Druckdifferenz aus Stagnations- und Hintergrunddruck. In bestimmten Bereichen treten, wie von Bobbert et al. mit der in dieser Arbeit verwendeten Methode der Natriumdotierung und Photoionisation gezeigt, bimodale Verteilungen auf, deren beide Maxima sich jeweils durch eine logarithmische Normalverteilung beschreiben lassen [32. Steigt der Druck, verschwindet das Maximum bei kleinen Clustergrößen wieder. Die Bildung größerer Cluster kann mit Hilfe einer Düse mit konischem Austrittskanal weiter verstärkt werden. Diese sorgt für eine größere Anzahl an Stößen als eine Lochdüse vergleichbaren Durchmessers. Zudem wird, ähnlich wie beim Skimmer, die Winkeldivergenz verringert und damit die Strahlintensität erhöht.

Die mittlere Clustergröße $\bar{N}$ lässt sich für eine Log-Normalverteilung über folgende empirische Formel bestimmen [32]:

$$
\bar{N}=D \cdot\left(\frac{\Gamma^{*}}{1000}\right)^{a}
$$

Die Parameter $D=11,6$ und $a=1,886$ wurden experimentell in der Arbeitsgruppe von Buck bestimmt. Der dimensionslose Parameter $\Gamma^{*}$ wurde von Hagena et al. eingeführt [53], um verschiedene Bedingungen zu klassifizieren. Dieser ist definiert über:

$$
\Gamma^{*}=\frac{\Gamma}{K_{c}}=\frac{n_{0} \cdot d_{e}^{q} \cdot T_{0}^{q-3}}{K_{c}}
$$

Die Konstante $K_{c}=\left(r_{c} \cdot T_{c}\right)^{q-3}$ setzt sich zusammen aus der Länge $r_{c}=$ $(m / \rho)^{1 / 3}$, wobei $m$ die Masse und $\rho$ die Dichte ist, und der Clustertemperatur $T_{c}=\Delta H_{\mathrm{sub}} / k_{B}$ bestehend aus der Boltzmann-Konstante $k_{\mathrm{B}}$ und der Sublimationsenthalpie $\Delta H_{\text {sub }}$. Die Werte, die Bobbert et al. für Wasser ermittelten, sind $T_{c}=5684 \mathrm{~K}, r_{c}=3,19 \AA$ und $q=0,63432$. Der Durchmesser der Düse $d_{e}$ ist 
gegeben durch $d_{e}=d / \tan (\alpha / 2)$, wobei $\alpha$ der Öffnungswinkel der konischen Düse ist. Die Teilchenzahl $n_{0}$ entspricht $n_{0}=p_{0} /\left(k_{B} \cdot T_{0}\right)$ mit den oben beschriebenen Anfangsbedingungen $p_{0}$ und $T_{0}$ bei einer Überschallexpansion.

\subsection{Wechselwirkungen von Molekülen}

In dem untersuchten System „Natrium-Wasser“ treten zwei Arten von Wechselwirkungen auf: Einerseits die zwischen den einzelnen Wassermolekülen, die Wasserstoffbrückenbindungen ausbilden, andererseits die (Teil-)Solvatation des Natriumatoms.

Eine Wasserstoffbrückenbindung $\mathrm{X}-\mathrm{H} \cdots \mathrm{Y}$ ist eine attraktive Wechselwirkung zwischen $\mathrm{X}-\mathrm{H}$ und $\mathrm{Y}$, bei der $\mathrm{X}$ eine größere Elektronegativität als das Wasserstoffatom aufweist [58]. Elektrostatische, induktive, dispersive und repulsive Kräfte haben Einfluss auf die Wasserstoffbrückenbindungen. Der jeweilige Anteil ist stark von der chemischen Umgebung und dem Abstand der Bindungspartner abhängig [59]. Bei größeren Abständen spielen die elektrostatischen Wechselwirkungen eine übergeordnete Rolle. Zu ihnen zählen sowohl die Anziehung zwischen Ionen als auch die Wechselwirkung verschiedener permanenter Multipole (Dipol-Dipol, Dipol-Quadrupol, Quadrupol-Quadrupol, etc.). Verringert sich der Abstand, kommen die induktiven und dispersiven Anteile hinzu. Beim induktiven Fall wird bei einem polarisierbaren Molekül ein Multipol durch die Annäherung eines Moleküls mit permanenten Multipol induziert. Im dispersiven Fall bilden zwei polarisierbare Moleküle spontan Multipole aus und ziehen sich so an. Bei geringen Abständen überwiegen die repulsiven Kräfte. Diese treten auf, wenn sich die Teilchen soweit angenähert haben, dass eine Überlappung der Orbitale stattfinden kann.

Die für diese Arbeit relevanten Wechselwirkungen umfassen auch die Beeinflussung eines H-Brückennetzwerkes durch ein Natriumatom. Ab einer Größe von ungefähr 15 Wassermonomereinheiten scheinen sich diese $\mathrm{Na}-\left(\mathrm{H}_{2} \mathrm{O}\right)_{n}$ Wechselwirkungen nicht mehr zu ändern. Eine eingehende Untersuchung dieses Phänomens hat gezeigt, dass eine grobe Einteilung in zwei Isomerklassen erfolgen kann [60. Diese unterscheiden sich im Abstand vom Elektron und dem Kontaktion Natrium, dem Grad der Solvatation und weisen unterschiedliche Ionisationsenergien (IE) auf. 
Die Bestimmung der IE erfolgt z.B. mit Hilfe einer Auftragung der Abhängigkeit der Ionenausbeute gegen die Photonenerergie, wie sie für die Clustergrößenscheibe $\mathrm{Na}\left(\mathrm{H}_{2} \mathrm{O}\right)_{45-450}$ in Abbildung 2.2 abgebildet ist. Die IE kann in dieser Auftragung mit der Methode des steilsten Gradienten bestimmt werden 61. Somit ergeben sich für Isomerklasse I, die als Kontaktionenpaar bezeichnet werden kann, 3,20 eV (ungefähr $387 \mathrm{~nm}$ ) 62,63 und für Isomerklasse II, die als solvenssepariertes Ionenpaar bezeichnet werden kann, $2,80 \mathrm{eV}[60$ als IE. Da die Ionenausbeute ab $3,3 \mathrm{eV}$ stetig steigt und bei $4 \mathrm{eV}$ um den Faktor 5 höher ${ }^{1}$ ist als bei $3,3 \mathrm{eV}$, ist anzunehmen, dass eine dritte Isomerklasse existiert, bei der das Natrium mit seinem 3s-Elektron, gebunden über induktive und dispersive Wechselwirkungen, auf der Oberfläche des Wasserclusters sitzt. Solche Strukturen mit Ionisationsenergien zwischen 3,4 und $4 \mathrm{eV}$ wurden bereits in kleinen natriumdotierten Wasser- und Methanolclustern nachgewiesen $40-42,64$. Der hohe Anteil der dritten Isomerklasse im Molekularstrahl ermöglicht eine signifikante Verstärkung des Photoionisationssignals durch vorgeschaltete IR-Anregung. Diese Methode wird in Abschnitt 2.3.1.2 beschrieben. Die genaue Bestimmung des Photoionisationssignals mit abnehmender UV-Vis Wellenlänge ist mit dem im Abschnitt 3.2.1 beschriebenen Lasersystem nun möglich und wird Bestandteil folgender Doktorarbeiten 65, 66 sein.

\subsubsection{Strukturen neutraler Wassercluster}

Für die Strukturaufklärung von Wasserclustern wurden bisher unterschiedliche experimentelle Methoden, wie zum Beispiel Elektronenbeugung [45], Infrarot[12, 14], Mikrowellen- 17] und Ramanspektroskopie [15 oder eine Verknüpfung von Streuexperimenten mit der in Abschnitt 2.3.1.1 beschriebenen IR(M)PDSpektroskopie unter Zuhilfenahme von quantenchemischen Rechnungen angewendet. Die Cluster bilden bis zu einer Größe von $n=10$ spezifische Minimumsstrukturen aus 11. Die Untersuchung der OH-Streckschwingungen bietet sich für die Strukturaufklärung an, da der OH-Oszillator je nach Bindungsmotiv und Umgebung in einem Cluster eine Verschiebung von mehr als $700 \mathrm{~cm}^{-1} \mathrm{im}$ Vergleich zum ungebundenen Oszillator erreichen kann 24.

Vom Monomer ist bekannt, dass es eine $C_{2 v}$-Symmetrie aufweist. Der

\footnotetext{
${ }^{1}$ Aus Skalierungsgründen nicht gezeigt.
} 


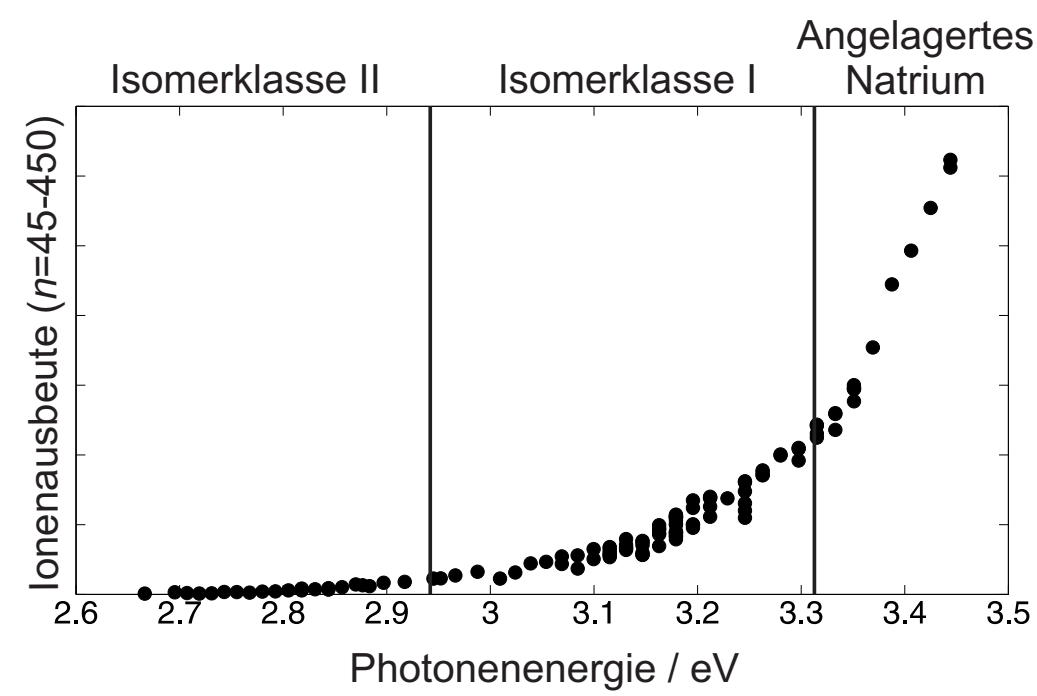

Abbildung 2.2.: Abhängigkeit der Ionenausbeute von der Photonenerergie für $\mathrm{Na}\left(\mathrm{H}_{2} \mathrm{O}\right)_{45-450}$-Cluster, verändert nach [41].

Gleichgewichts-HOH-Winkel beträgt $104,5^{\circ}$ und das Dipolmonent $\mu$ entlang der zweizähligen Drehachse 1,85 D [67]. Trimer bis Pentamer weisen zweidimensionale Ringstrukturen auf [15]. Das Hexamer besitzt eine dreidimensionale Käfigstruktur. Bei dieser Clustergröße treten je nach experimentellen Bedingungen drei unterschiedliche Strukturisomere auf $|17,68-70|$. Heptamer bis Decamer sind Abwandlungen der je nach Wasserstoffbrückentopologie $D_{2 d}$ oder $S_{4}$ symmetrischen Oktamerstruktur [24,71]. Bei größeren Clustern kommen andere, Massenspektrometrie-gebundene Methoden zur Strukturbestimmung zum Einsatz, um eine bessere Größenselektivität zu gewährleisten. Hilfreich für die Ionisation ist hierbei die Verwendung eines Chromophors wie Phenol (bis $n=49$ ) 36, 37 oder das in dieser Arbeit verwendete Natrium (Anwendungen bisher für $n \leq 56$ ) [38, 39|. Die Strukturzuordnung gestaltetet sich dabei allerdings sehr schwierig, da es in diesem Größenbereich eine Vielzahl isoenergetischer Isomere gibt [11.

Von den bisher publizierten Minimumsstrukturen ist bekannt, dass Vier- und Fünfringe bevorzugt ausgebildet werden 72,74 . Eine bis heute offene Fragestellung ist, ab welcher Größe ein vierfach koordiniertes Wassermolekül im Inneren eines Clusters auftritt. Hartke und Mitarbeiter konnten mit Hilfe des empirischen TTM2-F-Potentials den Clustergrößenbereich um $n=17-21$ 73 für das Auftreten dieses Strukturmerkmals identifizieren. Lagutschenkov et al. untersuchten mit 
Hilfe von harmonischen ab initio Rechnungen die Strukturen und Schwingungsspektren der oben genannten Clustergrößen [74|. Strukturvorschläge für größere Cluster bis hin zu $n=55$ wurden von Kazachenko et al. unter Zuhilfenahme verschiedener TIP4P Potentiale (TIP4P, TIP4P-Ew, TIP4P/2005), sowie TTM2.1-F und AMOEBA veröffentlicht [75]. Hartke und Mitarbeiter postulierten Strukturvorschläge bis $n=100$ auf Basis des TIP4P Potentials [72|. Simulierte IR-Spektren für große Wassercluster sind von Bowman und Mitarbeitern für einen amorphen und kristallinen $\left(\mathrm{H}_{2} \mathrm{O}\right)_{192}$-Cluster $|76|$, sowie von Buch et al. für Aggregate mit bis zu 931 Wassermolekülen veröffentlicht 44,77.

Ein Wassermolekül kann in einem Cluster bis zu vier Wasserstoffbrückenbindungen ausbilden, zwei als Donor (D) und zwei als Akzeptor (A). Die Koordinationsmöglichkeiten sind in Abb. 2.3 dargestellt. Diese Bindungsmotive haben für

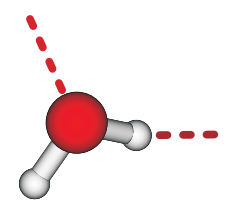

DA

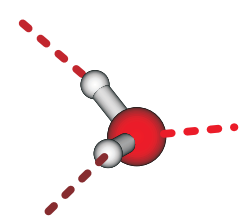

DDA

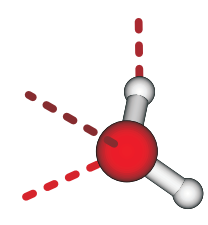

DAA

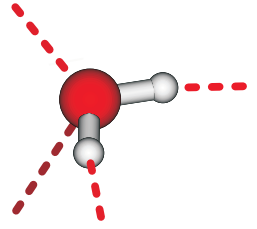

DDAA

Abbildung 2.3.: Koordinationsmöglichkeiten eines Wassermoleküls, verändert nach $78 \mid$.

Tabelle 2.1.: Position der Bandenmaxima der OH-Streckschwingungen bestimmter Bindungsmotive in Wasserclustern; Tabelle entnommen aus den Referenzen 41, 79, 80. Die Werte stammen aus den Arbeiten der Gruppen von Suhm, Buch, Buck und Fujii $99,15,24,37,39,44$ und gelten im Fall der zweifach und dreifach gebundenen Oszillatoren für Wassercluster bis $n=20$. Die Wellenzahlen des DA-Motivs stammen aus Gasphasenmessungen der Clustergrößen $n=2,3,4$ und 5 [15. Mit steigender Clustergröße findet eine zunehmende Überlappung der Banden statt [37].

\begin{tabular}{c||c|c|c|c|c}
\hline Motiv & DAA & DA $|\overline{15}|$ & DDAA & DDA & freie OH \\
\hline \hline & & 3602 & $3100-3500$ & & ca. 3720 \\
$\tilde{\nu} /$ & \multirow{3}{*}{$\leq 3200$} & 3533 & $3220($ Eis-Innen) & & (DA-Motiv) \\
$\mathrm{cm}^{-1}$ & & 3401 & $3400($ amorph/Eis-Außen) & $\geq 3450$ & ca. 3700 \\
& & 3355 & $3350($ Wert aus $|37|)$ & & (DAA-Motiv) \\
\hline
\end{tabular}


die bisher untersuchten Clustergrößen bis $n=20$ spezifische Positionen der Bandenmaxima, welche in Tabelle 2.1 aufgelistet sind [9, 24, 37, 39, 44. Bei größeren Clustergrößen findet eine zunehmende Überschneidung der Bereiche statt [37. Für die Beschreibung der Interaktion von Natrium und den Wasserclustern muss die Nomenklatur erweitert werden. Das Natrium wird über die freien Elektronenpaare eines Sauerstoffatoms koordiniert. Dieses Motiv wird mit „C“ bezeichnet. Oszilliert ein Wasserstoffatom des Wasserclusters in die delokalisierte Elektronenwolke des Na-3s-Elektrons, wird dieses Motiv mit „e“ deklariert [39].

\subsubsection{Aggregatzustände}

Makroskopisches Wasser tritt in unserer Alltagswelt in den drei Aggregatzuständen fest, flüssig und gasförmig auf. Bei Temperaturen über $374,12^{\circ} \mathrm{C}$ und einem Druck höher als 221,2 bar nimmt Wasser einen überkritischen Zustand ein. Die Phasenübergänge sind mit einer Umordnung der Wasserstoffbrückentopologie verbunden. Es sind bisher 17 verschiedene kristalline Formen von Eis experimentell nachgewiesen [81, 82. Die beiden relevanten Formen im Druckbereich bis 1 bar sind kubisches $\mathrm{I}_{\mathrm{c}}$ und hexagonales $\mathrm{I}_{\mathrm{h}}$ Eis. Sie sind durch IR-Spektroskopie kaum unterscheidbar [83], aber zum Beispiel durch Elektronenbeugung [45]. Die anderen Eis-Formen (II-XVI) enstehen erst bei Drücken von über 2 bar. Daneben gibt es drei Kategorien von amorphen, thermodynamisch metastabilen Eisstrukturen ohne Kristallgitteranordnung [84], die aufgrund ihrer Dichte im Verhältnis zum flüssigem Wasser in low, high und very high density amorphous (LDA, HDA und VHDA) eingeteilt werden. Die Bildung der einzelnen amorphen Spezies ist unterschiedlich. Während HDA und VHDA über die Komprimierung von LDA oder $\mathrm{I}_{\mathrm{h}}$ entstehen, wird LDA über die massive Abkühlung flüssiger Nanotröpfchen erzeugt 85. Die Abkühlrate liegt bei $10^{7}$ Kelvin pro Sekunde und ist damit (eher um eine halbe Größenordnung) kleiner als bei Überschallexpansionen mit Trägergasen [50]. In einer Studie von Bullock und Molinero wurde mit Hilfe von MD-Simulationen unter Verwendung des mW Modells, welches das Wassermolekül als Element zwischen Kohlenstoff und Silizium beschreibt [86], das Verhalten bei Abkühlung einer wässrigen Lösung mit 5\%,10\% und $15 \%$ gelösten Ionen untersucht. Bei der 10\% Lösung führte eine rapide Abkühlung von 10 Kelvin pro Nanosekunde zu Strukturen, die LDA ähneln. Erfolgte die Kühlung mit 1 Kel- 
vin pro Nanosekunde werden Strukturen mit 55\% Anteil an Eis im Verhältnis von $I_{c}$ zu $I_{h}$ von $2 \mathrm{zu} 1$ gebildet [87|. Die Formen LDA und HDA werden auch als glasartiges Wasser bezeichnet und stellen den Tieftemperaturbereich des Phasendiagramms von nicht kristallinem Wasser dar [5, 84. Dieses Phasendiagramm

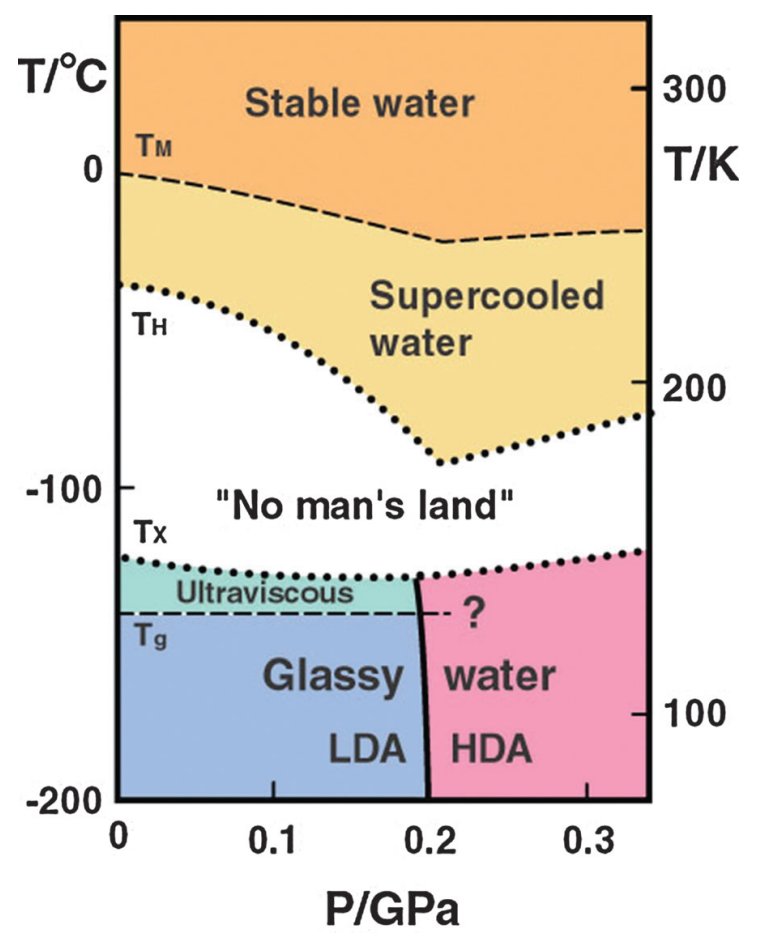

Abbildung 2.4.: Ausschnitt aus dem Phasendiagramm von nichtkristallinem Wasser. $\mathrm{T}_{\mathrm{M}}$ : Schmelztemperatur, $\mathrm{T}_{\mathrm{H}}$ : homogene Nukleationstemperatur, $\mathrm{T}_{\mathrm{X}}$ : Kristallisationstemperatur, $\mathrm{T}_{\mathrm{g}}$ : Übergangstemperatur von LDA zu ultraviscous water. Entnommen aus 84 .

ist in Abbildung 2.4 dargestellt. LDA vollzieht oberhalb von $T_{g}$ einen glasübergangsähnlichen Phasenwechsel zu ultraviscous water [88. Zwischen der homogenen Nukleationstemperatur $T_{H}$ und der Kristallisationstemperatur $T_{X}$, die den Übergang von glasartigem zu kristallinem Wasser beschreibt, sind aufgrund der rapiden Kristallisation nur Messungen von kristallinem Wasser möglich. Dieses Gebiet wird deshalb im Phasendiagramm von amorphem/flüssigem Wasser als "No man's land"“ bezeichnet [5,84,89. $\mathrm{T}_{\mathrm{M}}$ stellt die Schmelztemperatur dar.

Die voll größenselektive Bestimmung von Phasenübergängen bei Wasserclustern ist bisher nur für ionische Spezies möglich, da die verwendeten kalorimetrischen Techniken den Einfluss der Variation der verwendeten Energien und Temperatu- 
ren auf die Fragmentierung in einem Massenspektrometer untersuchen [90]. Bisher wurden Phasenübergänge für anionische Wassercluster der Größe $n=48$ bei $93 \pm 3 \mathrm{~K}$ und $n=118$ bei $118 \pm 3 \mathrm{~K}$ [91], sowie für kationische Wassercluster der Größen $n=60-79$ bei $133 \pm 6 \mathrm{~K} 90$ publiziert. Ob es sich bei diesen Übergängen um Schmelz- oder Glasübergänge handelt, lässt sich schwer interpretieren. Eine Zunahme der Übergangstemperatur mit steigender Clustergröße würde aufgrund der zunehmenden Schmelzwärme auf ein Schmelzen der Cluster hindeuten. Bei gleichbleibender Temperatur würde die Wärmekapazität pro Molekül abfallen, was zur These des Glasübergangs passt 90.

\subsection{Massenspektrometrie}

Eine Möglichkeit der Detektion von in einer Überschallexpansion erzeugten Clustern stellt die Massenspektrometrie dar. Im Wesentlichen besteht ein Massenspektrometer aus drei Bauteilen: Einer Ionenquelle, einem Analysator und einem Detektor. Für einen umfassenden Überblick in das Themengebiet sei hier auf die Literatur verwiesen 92,93 .

Die Ionisation kann mit verschiedenen Methoden erreicht werden. Grundsätzlich wird zwischen harten und weichen Ionisationstechniken unterschieden. Im ersten Fall wird dem System wesentlich mehr Energie zugeführt, als zur Ionisation benötigt wird. Diese Überschussenergie führt zur teilweisen Fragmentierung, welche molekül-/clusterspezifisch auftritt. Bei sanften Ionisationsmethoden findet diese Fragmentierung in der Regel nicht statt.

Die geladenen Teilchen werden mit Hilfe eines elektrischen oder magnetischen Feldes in Richtung Detektor beschleunigt. Die Trennung erfolgt aufgrund des unterschiedlichen Masse-zu-Ladung-Verhältnisses $(m / z)$. Meist werden drei Arten von Analysatoren verwendet. In einem Sektorfeldanalysator werden die Teilchen mit Hilfe eines Magnetfeldes auf eine Kreisbahn gelenkt, deren Radius abhängig ist vom $m / z$-Verhältnis. Die Rasterung der unterschiedlichen Verhältnisse wird mit Hilfe einer zunehmenden Feldstärke oder der Variation der Detektorposition gewährleistet. Eine Abwandlung dieser Technik stellt der Ionen Cyclotron Resonanz (ICR) Analysator dar. Dieser misst das $m / z$-Verhältnis in Abhängigkeit zur Cyclotronfrequenz in Gegenwart eines magnetischen Feldes. Die Frequenz kann mittels 
Fourier-Transformation in das $m / z$-Verhältnis umgewandelt werden [94]. In einem Multipolmassenfilter können die Teilchen mit Hilfe eines elektrischen Feldes separiert werden. Hierbei durchqueren die Teilchen ein Volumen, welches mit vier bzw. acht Stabelektroden ummantelt ist. Diese Filter können zu Tandem- oder zu Triple-Massenspektrometern zusammengefasst werden, in denen zuerst Separierung und anschließend spektroskopische Untersuchungen stattfinden [41. In einem Flugzeittrenner werden die Teilchen zunächst mit einem elektrischen Feld beschleunigt und durchqueren anschließend eine feldfreie Driftstrecke. Die Flugzeit ist dabei proportional zur Wurzel des $m / z$-Verhältnisses. Dieser Analysator bietet den Vorteil einer hohen Transmission und der gleichzeitigen Messung eines großen $m / z$-Bereichs.

Als Detektoren werden in den meisten Fällen Mikrokanalplatten (micro channal plates MCP) eingesetzt, auf die in Kapitel 3.1.3.3 näher eingegangen wird. Im Folgenden sollen verschiedene Ionisationsmethoden beschrieben sowie die Flugzeitmassentrennung näher erläutert werden.

\subsubsection{Ionisation}

Die Ionisation einer Spezies bei Energiezufuhr kann dann auftreten, wenn die aufgenommene Energie gleich groß oder größer ist als die Energiedifferenz zwischen dem ungeladenen und einfach positiv geladenen Teilchen. Die Ionisationsenergie (IE) (bzw. früher oft Ionisationspotential (IP)) bezeichnet dabei die Minimalenergie zwischen dem vibronischen Grundzustand des Atoms oder Moleküls und dem Vibrations-Grundzustand des erhaltenen Ions [92. Es gibt unterschiedliche Methoden, diese Energiebarriere zu überwinden. Liegt das Teilchen bereits in der Gasphase vor, stellen Stoßionisationsmethoden eine Möglichkeit dar. Eine harte Ionisationsform ist dabei die Elektronenstoßionisation (EI), bei der ein Elektron aus dem Atom/Molekül ein weiteres Elektron herausschlägt. Diese Methode wurde in der vorhandenen Apparatur angewendet und wird in Kapitel 3.1.3.1 erläutert. Anstelle eines Elektrons kann auch ein positiv geladenes Kation verwendet werden, das zuvor mit Hilfe von EI oder anderen Methoden erzeugt wurde. Diese sanfte Ionisationsform wird als chemische Ionisation (CI) bezeichnet.

Anionische, mit einem Elektron dotierte Cluster (z.B der Form $\left.\left(\mathrm{H}_{2} \mathrm{O}\right)_{n}^{-}\right)$, können mittels gepulster Gasentladung aus einem neutralen Clusterstrahl erzeugt wer- 
den $[95$. . Der in dieser Arbeit verwendete Ansatz der Natriumdotierung führt bei der Aufnahme des Natriums durch den Cluster zu einer Ionenpaarbildung $\mathrm{Na}^{+}$ und $\mathrm{e}^{-}$), die durch die Kombination mit der später beschriebenen Photoionisation ein der CI ähnelndes Verfahren der sanften Ionisation darstellt.

Liegt die Substanz in Lösung vor, so stellt die Elektrospray-Ionisation (ESI) eine Möglichkeit der Erzeugung von Ionen dar. Eine Anwendung für ESI ist die Analyse großer Biomoleküle [96, 97]. Die zu untersuchende Lösung wird durch eine Metallkapillare geleitet, an deren Spitze eine Spannung von $3-5 \mathrm{kV}$ angelegt ist. Aufgrund der Spannungsdifferenz zu einer Gegenelektrode expandieren geladene Aerosolpartikel über die Bildung eines Taylor-Kegels in Richtung Massenspektrometer. Dabei dampfen (aufgrund des hohen elektrischen Feldes) Lösungsmittelmoleküle ab. Durch die Verwendung eines gekreuzten Stickstoffgastroms kann das Abdampfen verstärkt werden. Es können sowohl positiv als auch negativ geladenen Ionen erzeugt werden. Diese Methode gilt als sanfte Ionisationsform und wird unter Atmosphärendruck durchgeführt. Über verschiedene Pumpstufen wird der Analyt mit Hilfe elektrisches Felder beschleunigt, fokussiert und in den Analysator geleitet.

Eine weitere Möglichkeit der Ionisation von schwer flüchtigen Substanzen stellt die Matrix-unterstütze Laser-Desorption/Ionisations-Methode (MALDI) dar 98, 99. Der Analyt wird in einer Matrix, meist aromatische Carbonsäuren, gelöst, wobei das Matrixmolekül in einem 100-bis 100000-fachen Überschuss vorliegt. Dieses Gemisch wird auf einer Oberfläche kristallisiert. Die Ionisation erfolgt mit Hilfe von stark fokussierten, nanosekunden-langen Laserpulsen. Das Matrixmolekül wird anhand des Absorptionsmaximums des Lasers ausgewählt. Meist finden frequenzverdreifachte und -vervierfachte Nd:YAG-Laser Verwendung. Nach der Absorption der Photonen dampfen Analyt und Matrixmoleküle von der Oberfläche ab. Dieser Prozess ist nicht selektiv. Es sind sowohl neutrale als auch ionisierte Partikel vorhanden, deren Größe von einzelnen Molekülen bis hin zu Partikeln schwankt. Die Matrixmoleküle protonieren die Analytmoleküle, die Trennung erfolgt über die oben angegebenen Analysatoren.

Eine direkte Photoionisation (PI) ist ebenfalls möglich und verschiedene Formen sollen im Folgenden beschrieben werden, um schließlich die in der Arbeit verwendete Infrarot-anregungsmodulierte, durch chemische Vorionisation verstärkte Photoionisation vorzustellen. 


\subsubsection{Photoionisation}

Die Energie $E$ eines Photons lässt sich über das Plancksche Wirkungsquantum $h$ und die Frequenz $\nu$ berechnen:

$$
E=h \cdot \nu
$$

Es wird unterschieden, ob die für die Ionisation benötige Energie über ein einzelnes Photon (SPI, single photon ionization) oder mehrere Photonen (MPI, multi photon ionization) bereitgestellt wird. SPI ist dabei unselektiv, da alle Stoffe, deren IE unterhalb der Photonenenergie liegt, ionisiert werden. Da die IE von Atomen zwischen 5 und $20 \mathrm{eV}(\lambda \leq 248 \mathrm{~nm})$ und von organischen Verbindungen zwischen 8 und $13 \mathrm{eV}(\lambda \leq 155 \mathrm{~nm})$ liegen [100], gibt es nur wenige geeignete Lichtquellen mit ausreichend hoher Photonendichte. Man bedient sich daher anderer Photoionisationsmethoden, um die IE zu überwinden. Eine stellt dabei die Resonanzverstärkte Mehrphotonenionisation (REMPI) dar. Der Unterschied zur nichtresonanten MPI besteht darin, dass reelle Zwischenzustände ausgenutzt werden, wodurch Ionisationswahrscheinlichkeit und Selektivität erhöht werden. Die gängige Notation für die unterschiedlichen REMPI-Prozesse beinhaltet zum einen die Anzahl $m$ der aufgenommenen Photonen bis zum Erreichen des Zwischenzustands und die Anzahl $n$ der Photonen von diesem Zustand bis zur Ionisation. Weicht die Wellenlänge von Anregung und Ionisation ab, wird die Anzahl mit $n^{\prime}$ bezeichnet. Eine Vielzahl von Substanzen von Stickstoffmonoxid 101 über aromatische Verbindungen 102 bis hin zu phenoldotierten Wasserclustern 36 kann anhand von REMPI in Massentrennern untersucht werden. Die Abbildung 2.5 stellt verschiedene REMPI-Prozesse nach obiger Notation schematisch dar. Der Fall 2.5 (d) entspricht einem IR/UV Doppelresonanzexperiment, bei dem der IR-Laser zeitlich ( $50 \mu \mathrm{s}$ bis $50 \mathrm{~ns}$ ) vor dem UV-Laser eingekoppelt wird. Dieses Experiment dient der Strukturaufklärung der neutralen Spezies X. Wird eine IR aktive Mode stimuliert, führt dies zu einer Entvölkerung des Grundzustands. Es folgt ein Signalabfall, da weniger Ionen mit Hilfe des R2PI Prozesses gebildet und detektiert werden. Diese Methode wurde von Zwier et al. als RIDIR (resonant iondip infrared spectroscopy) bezeichnet [103]. Die Nutzung der durch IR-Photonen induzierten Fragmentierung, meist von ionischen Spezies zur Generierung von Infrarotspektren, geht auf die Arbeiten von Lee und Mitarbeitern zurück und wird als IR(M)PD (Infrared (multi)photon dissociation) bezeichnet 20-22]. 


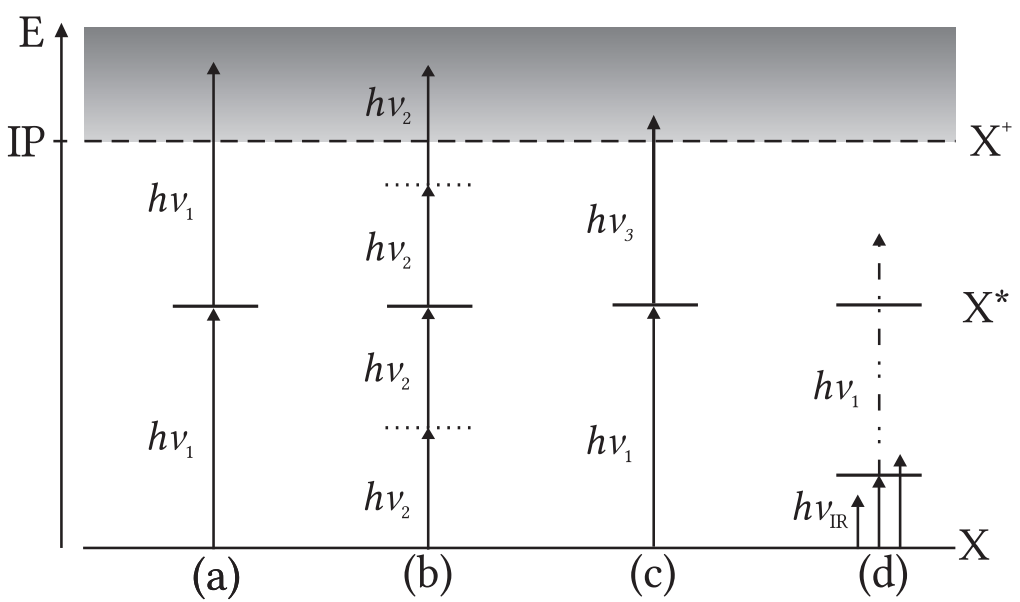

Abbildung 2.5.: Schematisches Energiediagramm für verschiedene REMPIProzesse mit reellen (-) und virtuellen $(\cdots)$ Zwischenzuständen; (a) $(1+1) R 2 P I$, (b) $(2+2) R 4 P I,(c)\left(1+1^{\prime}\right) R 2 P I$. Das Energieschema (d) zeigt eine IR-Anregung vor UV-Anregung. Weitere Erläuterungen im Text. Entnommen und modifiziert nach [80].

\subsubsection{IR-anregungsmodulierte, durch chemische Vorionisation verstärkte Photoionisationsspektroskopie}

Die in der Arbeit verwendete Ionisationsmethode, die im Folgenden als IRCIPI abgekürzt wird, stellt in gewisser Weise eine Mischung aus chemischer Vorionisation und Photoionisation dar und wurde bereits in der Diplomarbeit von Florian Zurheide [79], der Masterarbeit von Christoph Dierking [80|, sowie den Publikationen [11, 47] vorgestellt und diskutiert. Die Erzeugung der OHStreckschwingungsspektren ähnelt der RIDIR-Methode mit dem Unterschied, dass das IR-Signal aus einer Zunahme der ionisierten Spezies im Massenspektrum resultiert.

In Abbildung 2.6 ist die Ionisationsmethode IR-CIPI mit einem vereinfachten experimentellen Aufbau, der im nächsten Kapitel beschrieben wird, dargestellt. Das Zeitfenster für die chemische Vorionisation der Cluster beträgt bei einem Molekularstrahl mit Helium als Trägergas ungefähr $240 \mu$ s [47]. Die Reaktion kann allerdings auch instantan bei der Aufnahme des Natriums erfolgen. Angenommen wird für das Zeitfenster eine mittlere Strahlgeschwindigkeit von $1500 \mathrm{~m} / \mathrm{s}$. Dieser, eine chemische Reaktion des Natriums in Lösungsmittel einleitende Prozess, wird in der Literatur auch als Ionisation des Natriums bezeichnet 104,105] und 


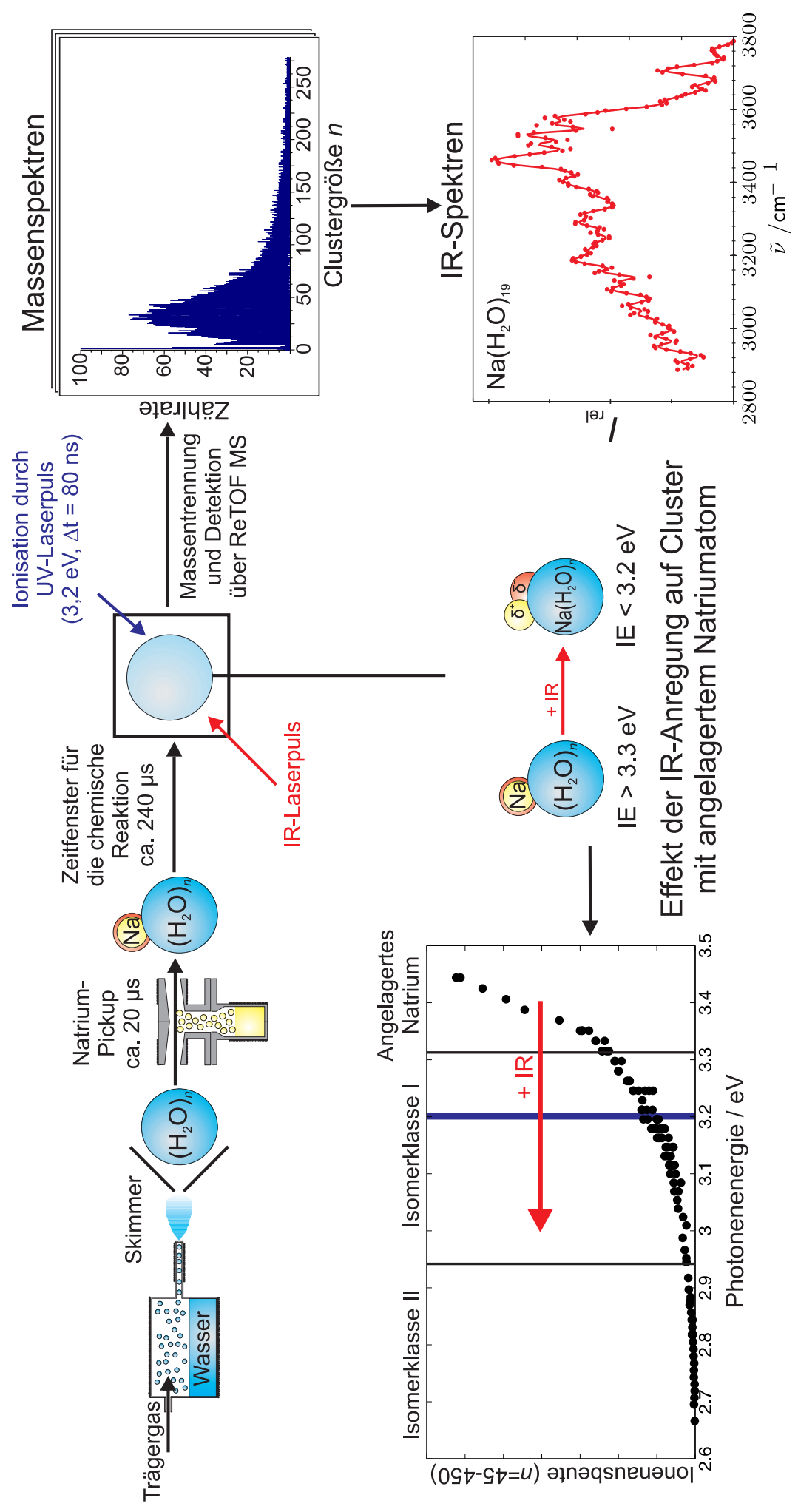

Abbildung 2.6.: Schema der IR-CIPI Methode. Erläuterungen im Text. Verändert nach 79. 
führt, wie bereits in Abschnitt 2.2 erwähnt, zu einer Absenkung der IE im Falle von Wasserclustern auf ca. 3,0-3,3 eV (ungefähr 413-376 nm) für Isomerklasse I (ab $n=4) 62,63$ und ca. 2,6-3,0 eV (ungefähr 477-413 nm) für Isomerklasse II $($ ab $n=15)[60$, sodass eine direkte Photoionisation mit gängigen UV/VisLasersystemen möglich ist. Um die strukturellen Informationen der Wassercluster zu erhalten, deren Wasserstoffbrückentopologie den geringsten Störungen durch das Natrium unterliegt, wird die UV/Vis-Wellenlänge im oberen Frequenzbereich der Isomerklasse I eingestellt und der IR-Laser $80 \mathrm{~ns}$ vor dem Photoionisationslaser eingekoppelt. Die Annahme ist, dass die IE umso höher liegt, je geringer die Wechselwirkung des Natrium mit den Wasserclustern ausfällt. Dieses Verhalten wurde in quantenchemischen Rechnungen für Na-Wassercluster am Beispiel der Größe $n=15$ eingehend untersucht [60]. Wird eine IR-Bande stimuliert führt dies zu einer Umwandlung der Cluster mit außen angelagertem Natrium. Die durch IR-Photonen aufgeheizten Cluster haben dann eine geringere IE und erhöhen so die Ionenausbeute im Vergleich zum gewöhnlichen Photoionisationsexperiment. Die Plausibilität dieses Mechanismus ist durch die Reaktion von Natrium mit Eisoberflächen begründet. Diese setzt über die Bildung der Ionenpaare $\mathrm{Na}^{+}$und $\mathrm{e}^{-}$ ab einer Temperatur von ca. $95 \mathrm{~K}$ ein [106, 107. Die IR-Anregung erzeugt auch für große Cluster einen signifikanten Temperaturanstieg in diesem Bereich.

Die Effizienz dieses Mechanismus wird begünstigt durch ein hohe Teilchendichte an Clustern mit angelagertem Natrium im Molekularstrahl, welche die positiv signalwirksame Absorption der IR-Photonen maximieren. Das niedrige Referenzsignal erhöht die erreichbare Signalzunahme. Außerdem unterdrückt die schwellennahe Photoionisation die Fragmentierung der Cluster 11. Diese Methode wurde bisher auf die Systeme Natrium Wasser/Methanol angewendet, ist aber für andere wasserstoffbrückengebundene Systeme und Alkalimetalle denkbar 41, 42. Durch die Kopplung mit einem Flugzeitmassentrenner kann durch einen Scan der IR-Wellenlängen das Absorptionsspektrum aller im Molekularstrahl befindlichen Clustergrößen aufgenommen werden. Ein Zeitfenster von $80 \mathrm{~ns}$ zwischen IR- und UV/Vis-Puls wurde gewählt, um die IR-Photonen effektiv zu nutzen und ergibt sich aus den Pulsdauern der verwendeten Lasersysteme ( $\leq 8 \mathrm{~ns}$ für IR bzw. $\leq 30 \mathrm{~ns}$ für UV/Vis), sowie der Fluktuation des Excimerlaser-Pulses (vgl. Kapitel 3.2.1). 


\subsubsection{Flugzeitmassentrennung}

Der Aufbau eines einfachen, linearen Massenspektrometers ist schematisch in Abb. 2.7 gezeigt. Nach der Ionisation der Spezies erfolgt eine Beschleunigung der Teilchen mit Hilfe von elektrischen Feldern. Die elektrische Energie $E_{\text {el }}$, welche

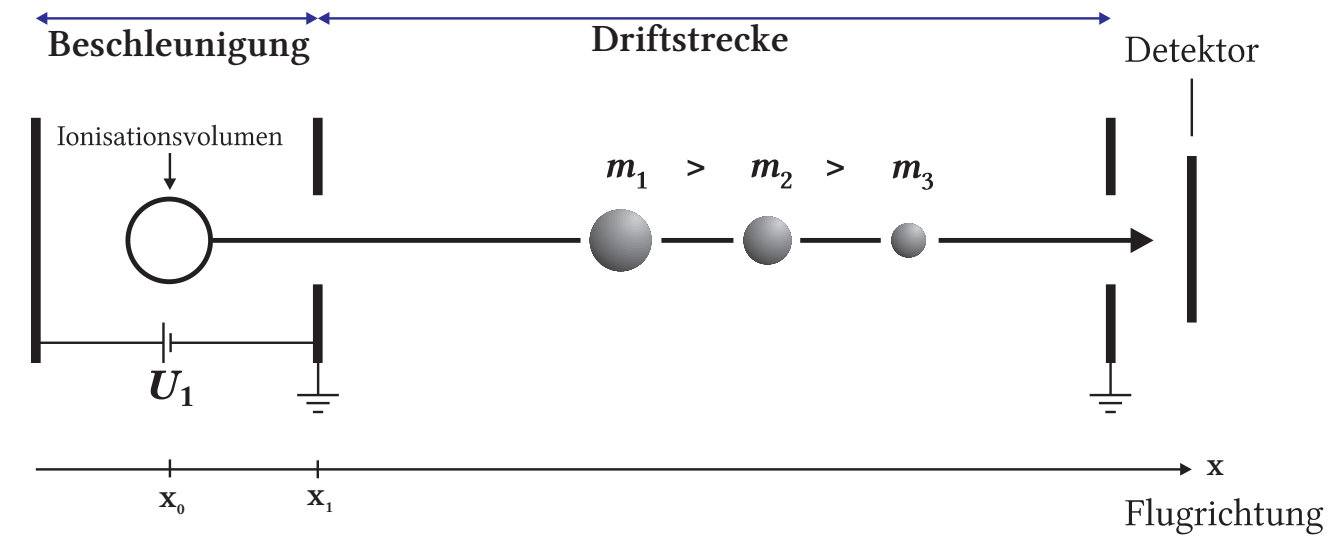

Abbildung 2.7.: Schematischer Aufbau eines linearen Flugzeit-Massenspektrometers, entnommen aus |108|.

die Teilchen in dieser Beschleunigung erfahren, ist abhängig von ihrer Ladungszahl $z$, der Elementarladung $e$ und der Spannungsdifferenz $\Delta U_{x_{0}}$ zwischen dem Entstehungsort und der Beschleunigungselektrode.

$$
E_{\mathrm{el}}=z \cdot e \cdot \Delta U_{x_{0}}
$$

Unter der Annahme, dass diese Energie vollständig in kinetische Energie umgewandelt wird, ergibt sich:

$$
z \cdot e \cdot \Delta U_{x_{0}}=1 / 2 \cdot m \cdot v^{2}
$$

Für die Geschwindigkeit $v$ gilt unter der Annahme, dass zu Beginn der Ionisation alle Teilchen die Anfangsgeschwindigkeit $v_{0}=0$ haben:

$$
v=\sqrt{\frac{2 \cdot e \cdot \Delta U_{x_{0}}}{m / z}}
$$


Aufgrund des unterschiedlichen $m / z$-Verhältnisses ergeben sich somit unterschiedliche Geschwindigkeiten. Es ergibt sich für die Flugzeit $t_{\text {Drift }}$ in der feldfreien Driftstrecke $L$ :

$$
t_{\text {Drift }}=\frac{L}{\sqrt{2 \cdot e \cdot \Delta U_{x_{0}}}} \cdot \sqrt{\frac{m}{z}}
$$

Im Falle der Ionisation durch IR-CIPI kann angenommen werden, dass $z=1$ ist. Beim Durchqueren der feldfreien Driftstrecke erfolgt dann eine Trennung der Teilchen. Spezies mit geringerer Masse erreichen den Detektor früher als jene mit größerer Masse. Die Charakterisierung eines Massenspektrometers erfolgt über die Halbwertsbreiten $\Delta m_{\mathrm{FWHM}}$, die das Aufösungsvermögen $A|92|$ definieren:

$$
A:=\frac{m}{2 \Delta m_{\mathrm{FWHM}}}
$$

Verschiedene Effekte begrenzen das Auflösungsvermögen. Erfolgt die Ionisation mit einem Laserpuls, so hat dieser eine gewisse Dauer und Ortsauflösung, was Auswirkungen auf die Beschleunigungsstrecke hat und zu einer Signalverbreiterung führt. Gleiches gilt, aufgrund der baubedingten Anstiegszeiten, für die verwendeten Detektoren. Außerdem weisen die zu ionisierenden Teilchen eine Geschwindigkeitsverteilung auf. Durch Modifizierung des Aufbaus aus Abb. 2.7 kann eine Verbesserung des begrenzten Auflösungsvermögens erfolgen.

Für die Minimierung der oben genannten Fehler sind die Ortsfokusbedingungen relevant. Der Ortsfokus n-ter Ordnung ist definiert über:

$$
\frac{\partial^{n} t}{\partial x_{0}^{n}}=0
$$

Den Ortsfokus erster Ordnung erreichen die Teilchen gleicher Masse mit unterschiedlichen Anfangsgeschwindigkeiten zur gleichen Zeit. Dieser ist beim Aufbau in Abb. 2.7 bereits nach der doppelten Beschleunigungsstrecke erreicht. Eine sinnvolle Massenauflösung ist dann nicht gewährleistet. Durch die Verwendung eines zweiten Beschleunigungsfeldes, wie von Wiley und McLaren 1955 postuliert [109], kann der Ortsfokus durch Variation der beiden Beschleunigungsfelder entlang der Driftstrecke verschoben werden. Die Felder können auch dafür genutzt werden einen Ortsfokus zweiter Ordnung zu generieren. Dieser ist massenabhängig und minimiert den Geschwindigkeitsfehler, jedoch ist er ebenfalls nach einer gerin- 
gen Flugstrecke erreicht. Die Verwendung eines Reflektrons, wie es in Abbildung

Ionisations-

volumen

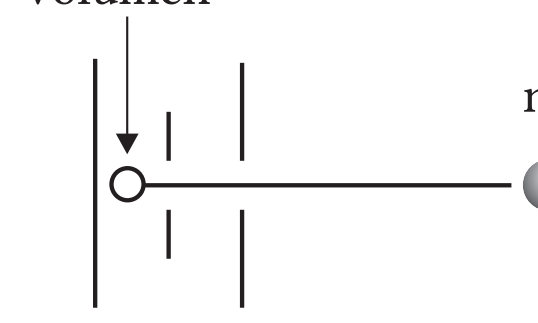

Beschleunigung

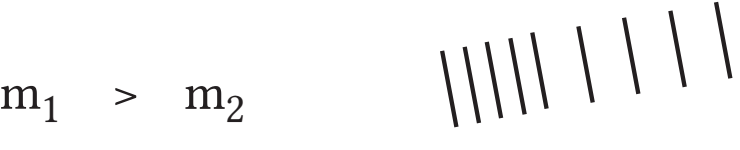

kl. Ekin

gr. $\mathrm{E}_{\mathrm{kin}}$

Reflektor

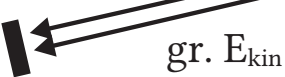

Detektor

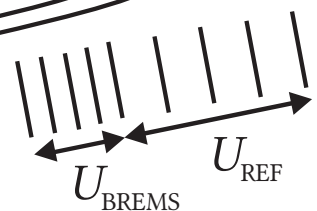

Abbildung 2.8.: Schematischer Aufbau eines Reflektron FlugzeitMassenspektrometers, verändert nach 108.

2.8 schematisch dargestellt ist, verlängert die Flugstrecke. Die Entwicklung dieses Bauteils erfolgte durch Mamyrin und Mitarbeiter Ende der 70er Jahren 110,111. Das Reflektron besteht aus mindestens einem elektrischen Feld, dessen Aufbau der Ionenquelle nachempfunden ist. Die Ionenpakete werden zunächst über die Bremsspannung $U_{\text {BREMS }}$ abgebremst und anschließend mit Hilfe von $U_{\text {REF }}$ reflektiert. Schnelle Ionen tauchen so weiter ins elektrische Feld ein als langsame und verbringen mehr Zeit im Reflektron. Über die Variation der elektrischen Felder ist es möglich, den zweiten Ortsfokus in die Detektorebene zu legen. Die Simulation der elektrischen Felder beim Bau oder bei Modifikation eines ReTOF-MS kann mit Programmen wie z.B. Simion durchgeführt werden [112].

\subsection{Quantenchemische Rechnungen}

Eine Möglichkeit der Validierung experimenteller Ergebnisse stellen quantenchemische Rechnungen dar. Eine detaillierte Beschreibung der Grundlagen der Quantenchemie kann der Referenz [113] entnommen werden. Bei dieser Form der Rechnungen müssen zunächst vorgegebene Strukturen optimiert und deren Energie bestimmt werden. Ein in dieser Hinsicht besonders effizientes und benutzerfreundliches Programmpaket stellt Gaussian09 [114] dar, das über Suchalgorithmen die lokalen Minima auf einer Potentialhyperfläche bestimmt. Eine vorgegebene Struk- 
tur wird dabei (bei optionalem Erhalt der Symmetrie) so lange optimiert, bis Energie und Energiegradient den festgelegten Konvergenzkriterien entsprechen. Aufgrund der Komplexität der Potentialhyperfläche von Clustern sind die bestimmten Minima als lokale Minima anzusehen. Durch die Variation der Strukturen und Vergleich der erhaltenen Energien können globale Minima bestimmt werden. Da das Programmpaket während der Optimierung nicht zwischen lokalen Minima und Sattelpunkten unterscheidet, kann eine harmonische oder anharmonische Kraftfeldrechnung durchgeführt werden. Liegen imaginäre Anregungsfrequenzen vor, so kann davon ausgegangen werden, dass sich die Struktur in einem Sattelpunkt befindet oder der Gradient nicht verschwindet. Aus den Frequenzen der IR-Absorption werden die Nullpunktsenergien bestimmt. Die von Gaussian erhaltenen Ergebnisse sind Näherungslösungen der Schrödingergleichung, welche bei Mehrelektronensystemen aufgrund der Elektronenkorrelation nicht mehr analytisch gelöst wird:

$$
\hat{H} \cdot \Psi=E \cdot \Psi
$$

bei der $E$ die von Gaussian berechnete Energie, $\Psi$ eine Wellenfunktion und $\hat{H}$ der Hamiltonoperator, bestehend aus dem Operator der kinetischen Energie $\hat{T}$ und potentiellen Energie $\hat{V}$, ist. Für die Lösung dieser Gleichung stehen dem Programmpaket Gaussian unterschiedliche Rechenmethoden und Basissätze zur Verfügung, auf die in den folgenden Abschnitten eingegangen wird.

\subsubsection{Methoden}

Die einfachste $a b$ initio Rechenmethode stellt die Hartree-Fock-Methode (HF) dar. Diese betrachtet die Wechselwirkung eines Elektrons mit der mittleren Elektronendichte der verbleibenden. Eine Erweiterung ist der störungstheoretische Ansatz 2. Ordnung nach Møller und Plesset (MP2), der zusätzlich die Elektronenkorrelation näherungsweise berücksichtigt. Sie ist aufgrund der langsamen Konvergenz bezogen auf die Rechenleistung aufwendig. Die Becke 3-Parameter-Lee-YangParr-Methode (B3LYP) erweitert HF durch ein empirisches Funktional zur Beschreibung der Elektronenkorrelation und Austauschwechselwirkung. Sie stellt ein Hybridfunktional aus der Dichtefunktionaltheorie nach Becke mit 3 Parametern (B3) und dem Austauschfunktional nach Lee, Ynag und Parr (LYP) dar. 


\subsubsection{Basissätze}

Durch den Basissatz werden die mathematischen Funktionen für eine Rechenmethode definiert. In den meisten Fällen werden die Orbitale dabei als Gaussfunktionen angenähert, diese Funktionen werden als Gaussian Type Orbital (GTO) bezeichnet. Der Vorteil zu den genaueren Slater Type Orbitals (STO) ist eine einfachere Berechnung der Integrale und Kombinationen mehrerer Funktionen.

Für die quantenchemischen Rechnungen dieser Arbeit wurden die Pople-Basissätze der Form 6-31+G(d,p) bzw. 6-31+G** verwendet. Dabei steht die „6“ für die Anzahl der Gaussfunktionen, durch welche die Rumpforbitale beschrieben werden. Die Ziffern „31“ stehen für die Anzahl der Gaussfunktionskomponenten (3 bzw. 1), die für die Basisfunktionen der Valenzelektronen zusammengefasst werden. Durch "++ werden diffuse Funktionen der schweren Atome ( $s$ - und $p$-Funktionen) eingeführt. Die Parameter „,(d,p)“ bzw. , "**“ bewirken, dass beim Wasserstoff zusätzlich ein p-Orbital und bei schwereren Atomen ein d-Orbital eingesetzt werden.

Ein vergleichbarer Dunning-Basissatz, wie er häufig für Wassercluster in Verbindung mit MP2-Rechenmethoden verwendet wird, wäre aug-cc-pVDZ 74, 115]. Das „pVDZ“ steht für einen polarisierbaren Valence-Double-Basissatz. Mit „cc“ wird gekennzeichnet, dass es sich um einen korrelationskonsistenten Basissatz handelt, der zusätzlich um eine diffuse Funktion pro Drehimpulsquantenzahl (als „aug“ bezeichnet) erweitert wird. Der Vorteil dieser Form der Basissätze ist, dass die berechneten Eigenschaften systematisch mit der Erweiterung der Basisfunktionen konvergieren (pVDZ $<$ pVTZ $<$ pVQZ $<$ pV5Z $<$ pV6Z $<$ CBS).

\subsubsection{Modellpotentiale}

Bei großen Wasserclustern ab ungefähr $n=20$ bedient man sich aufgrund des hohen Rechenaufwands Modellpotentialen für die Strukturoptimierung, die man der TIP- [116] und TTM-Klasse [117-120 zuordnen kann. Die gängigsten sind TIP4P ( Transferable Intermolecular Potential-4 Point) [116] und TTM3-F (Thole-type model, v.3.0, flexible) [120]. Die Potentiale haben, begründet durch die unterschiedlichen Problemstellungen, an die sie angepasst sind, andere Parametrisierungen. Während TIP4P für die Beschreibung der Struktur und Thermodynamik von Lösungen entwickelt wurde, ist TTM-2F, der Vorgänger von TTM-3F, an die $a b$ initio Daten von Wasserclustern (hautsächlich Dimere) angepasst. TTM3-F 
stellt eine Erweiterung für die Beschreibung der flüssigen Phase dar [120]. TIP4P und die TTM-Potentiale beschreiben das Monomer des Wassers mit Hilfe von vier Zentren, wobei drei durch die Lage der Atome definiert sind und das vierte, ein masseloses M-Zentrum, längs der Winkelhalbierenden des HOH-Bindungswinkels angeordnet ist. Bis auf den Sauerstoff wird jedem der Zentren eine Partialladung zugeordnet. Das TIP4P-Potential betrachtet dabei die Moleküle als starre Dipole mit festem OH-Bindungsabstand und HOH-Winkel. Mit zusätzlichen induzierten Dipolen und Massen an den Sauerstoff- und Wasserstoffatomen reproduziert das immer noch starre TTM2-R ( rigid=starr) besser als TIP4P das Dipol- und Quadrupolmoment des Monomers 117. Die Weiterentwicklung TTM-2F modelliert das Wassermonomer in Bezug auf OH-Bindungsabstand und HOH-Winkel flexibel und ergänzt die intramolekulare Potentialhyperfläche um eine nichtlineare Dipolmomentfläche [118]. TTM-3F reduziert die Polarisierbarkeit des Monomers auf das massenlose M-Zentrum und fügt zusätzlich dem Monomer eine phänomenologische Dipolmomentfläche hinzu, was zu einer besseren Vorhersage der IR-Spektren von Gasphase und Flüssigkeit führt 120]. Die Erweiterung der Parametrisierung von starren, wechselwirkenden Punktladungen zu flexiblen, polarisierbaren Teilchen geht mit einer Erhöhung der Rechenzeit einher. Die Optimierung eines Clusters der Größe $n=20$ dauert unter Verwendung des TTM-2F Potentials 20 mal länger als mit TIP4P 73 . 


\section{Versuchsaufbau und experimentelle Methoden}

Dieses Kapitel beschreibt den Aufbau der in der vorliegenden Arbeit verwendeten Molekularstrahlapparatur. Die Konzeption der Apparatur erfolgte Anfang der 1990er Jahre während der Doktorarbeit von Dr. Martin Hobein [121] in der Arbeitsgruppe von Prof. Dr. Udo Buck am Max-Planck-Institut für Strömungsforschung in Göttingen. Dr. Stefan Schütte führte während seiner Doktorarbeit die ersten erfolgreichen Messungen Mitte der 90er Jahre an Edelgasclustern [122] durch. Der Umzug in das Institut für Physikalische Chemie der Universität Göttingen erfolgte Anfang 2005.

Da eine detaillierte Beschreibung der Apparatur bereits in vorherigen Arbeiten erfolgte [46, 121 124], ist hier nur eine kurze Zusammenfassung der einzelnen Bestandteile gegeben. Auf Modifikationen, wie z.B die Implementierung des neuen durchstimmbaren UV/Vis-Lasersystems, wird näher eingegangen.

\subsection{Experimenteller Aufbau}

Die Molekularstrahlapparatur besteht aus drei mit Ventilen voneinander getrennten Vakuumkammern, die differentiell gepumpt werden. Eine schematische Darstellung ist in Abbildung 3.1 enthalten. In der Expansionskammer wird der Molekularstrahl mittels adiabatischer Überschallexpansion erzeugt. Über einen Skimmer mit $600 \mu \mathrm{m}$ Durchmesser gelangt er in die Zwischenkammer, in der sich die Pickup-Zelle befindet, und wird dort mit Natrium dotiert. Anschließend dringt der Strahl durch ein UHV-Ventil in die Detektionskammer ein. In dieser befindet sich ein Flugzeitmassenspektrometer mit Reflektron. 


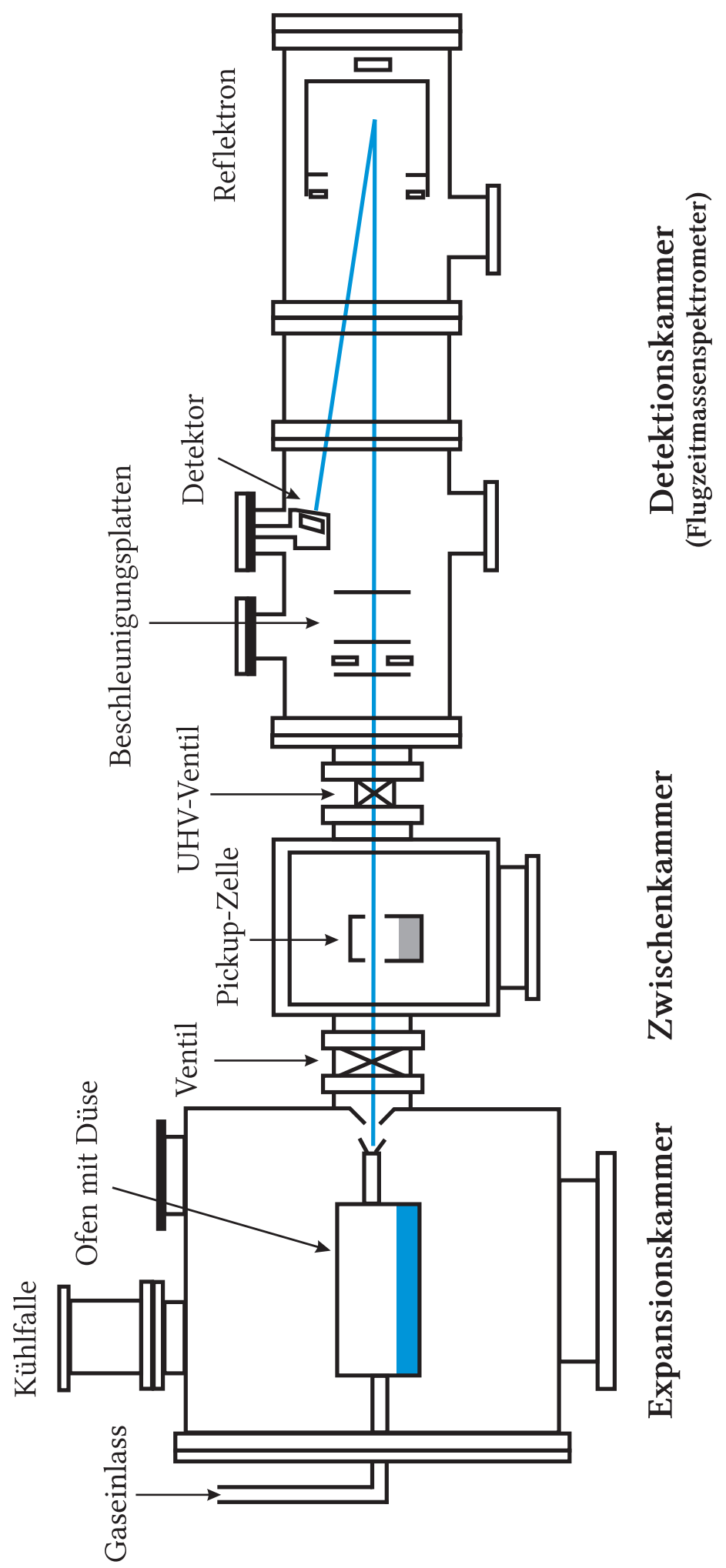

Abbildung 3.1.: Schematischer Aufbau der Molekularstrahlapperatur. 


\subsubsection{Expansionskammer}

Die Expansionskammer enthält die Molekularstrahlquelle. Diese besteht aus einem Edelstahlzylinder (im Weiteren als Ofen bezeichnet) mit aufgeschraubter Düsenhalterung samt Düsenplatte. Die Befüllung ist über einen Gaseinlass von außen möglich. Dieser ermöglicht auch die Verwendung von bei Raumtemperatur gasförmigen Substanzen. Ofen und Düse sind separat über einen Widerstandsdraht in einem Temperaturbereich von $40{ }^{\circ} \mathrm{C}$ bis $200{ }^{\circ} \mathrm{C}$ beheizbar. Die Begrenzung des Temperaturbereichs ist einerseits durch die Wärmeabgabe der Öldiffusionspumpe (vgl. Abschnitt 3.1 .4 und andererseits durch die thermische Stabilität der verwendeten FKM (Viton) Dichtringe gegeben (vgl. [41]). Die Temperatur wird über Thermoelemente erfasst, welche über PID-Regler, die proportionales $(\mathrm{P})$, integrales (I) und differentielles (D) Regelverhalten für die Heizströme kombinieren (vgl. [46]), die Gleichspannungsstromquellen $(<30 \mathrm{~V})$ steuern. Die Temperatur der Düse wird stets etwas höher gewählt, um eine Kondensation der Substanz in der Düse zu verhindern. Bereits kleinste Verunreinigungen in der Düse führen zu einer veränderten Größenverteilung. Als Indiz für das allmähliche Zusetzen der Düse kann die Abnahme des Hintergrunddrucks in der Expansionskammer verwendet werden. Dieser wird mit Hilfe einer Penning-Röhre ermittelt.

Es stehen zwei Ofenkonstruktionen zur Verfügung, die sich durch ihre Innenvolumina unterscheiden $\left(V \approx 150 \mathrm{~cm}^{3}\right.$ und $V \approx 250 \mathrm{~cm}^{3}$ ), sowie zwei Düsenhalterungen mit Längen von $40 \mathrm{~mm}$ und $73 \mathrm{~mm}$. Beide Öfen können über einen abnehmbaren Flansch geöffnet werden, was das Reinigen vereinfacht. Für die in dieser Arbeit vorgestellten Messungen wurde eine konische Düsenplatte mit einem Öffnungsdurchmesser von $65 \mu \mathrm{m}$ und einem Öffnungswinkel von $40^{\circ}$ verwendet.

Die Position der Molekularstrahlquelle lässt sich von außen über Stellräder mit Schrittzählern in xyz-Richtung verstellen, wobei die z-Richtung der Flugrichtung des Molekularstrahls entspricht. Eine Neuausrichtung des Ofens muss nur nach Ausbau oder Wechsel erfolgen. Bei der Reinigung der Düse bleibt die Position erhalten. Die Grobausrichtung des Ofens in xy-Richtung erfolgt mittels Messung des Druckanstiegs am Ionisationsvakuumeter in der Zwischenkammer. Hierfür muss die Molekularstrahlquelle in z-Richtung dicht am Skimmer platziert werden. Die Feinjustage erfolgt iterativ über die gemessene Größenverteilung. 


\subsubsection{Zwischenkammer}

Die Zwischenkammer beinhaltet die Pickup-Zelle und dient ebenfalls als differentielle Pumpstufe, um einen zu starken Druckanstieg in der Detektionskammer zu verhindern. Die Zelle besteht aus zwei Teilen, dem Reservoir, welches mit dem Dopanden befüllt werden kann (in den Messungen zu dieser Arbeit wurde Natrium verwendet) und einem Kopf, durch den der Molekularstrahl geleitet wird. Ein schematischer Aufbau ist in Abbildung 3.2 zu finden. Es stehen zwei Pickup-ZellenAnordnungen zur Verfügung (s. Abbildung 3.3). Sie unterscheiden sich durch die Position in der Kammer bezogen auf die Molekularstrahlachse und durch die Länge des Kopfes (43 mm und $54 \mathrm{~mm}$ ) 41]. Kopf und Reservoir können, ähnlich wie die Temperierung der Molekularstrahlquelle, separat über eine Widerstandsheizung geheizt werden. Die kleinen Zellen wurden für einen Betrieb mit zwei unterschiedlichen Dopanden konstruiert [46]. Der Molekularstrahl könnte dann zuerst mit einer schwerflüchtigen, organischen Substanz (z.B. große Alkohole) und anschließend mit dem Chromophor Natrium dotiert werden. Erste Tests der DoppelpickupZellen-Anordnung wurden während der Masterarbeit von Christoph Pradzynski durchgeführt 78. Hierbei wurden Wassercluster zuerst mit Natrium und anschließend mit 1-Butyl-3-Methyl-Imidazol-Trifluormethansulfonat, einer ionischen Flüssigkeit, dotiert. Es konnte jedoch lediglich eine Zunahme des Signals des Kations der ionischen Flüssigkeit mit steigender Reservoirtemperatur festgestellt werden. Die Messungen der hier vorgestellten IR-Spektren natriumdotierter Wassercluster erfolgten mit der großen Pickup-Zelle, da bereits in vorhergehenden Arbeiten festgestellt wurde, dass diese konstantere Bedingungen liefert 41, 125.

Von außen lässt sich die Zelle in vertikaler und horizontaler Ebene zum Molekularstrahl über Stellräder mit Schrittzählern verstellen. Für die Justage nach Ausbau und Reinigung gibt es zwei Möglichkeiten. Eine Grobjustage kann im Vakuum bei geöffneten Ventilen zwischen Expansionskammer, Zwischenkammer und Detektionskammer erfolgen. Es kann ein leichter Druckanstieg/-abfall in der Detektionskammer beobachtet werden, je nachdem ob der Strahl frei die PickupZelle passieren kann oder nicht. Eine Feinjustage erfolgt dann über das gemessene Massenspektrum. Da jedoch die Druckschwankungen sehr gering sind, empfiehlt sich die zweite Methode. Bei dieser wird die Expansionskammer belüftet und ein Laserpointer durch den Skimmer geschickt, sodass er auf die Öffnung des UHV- 


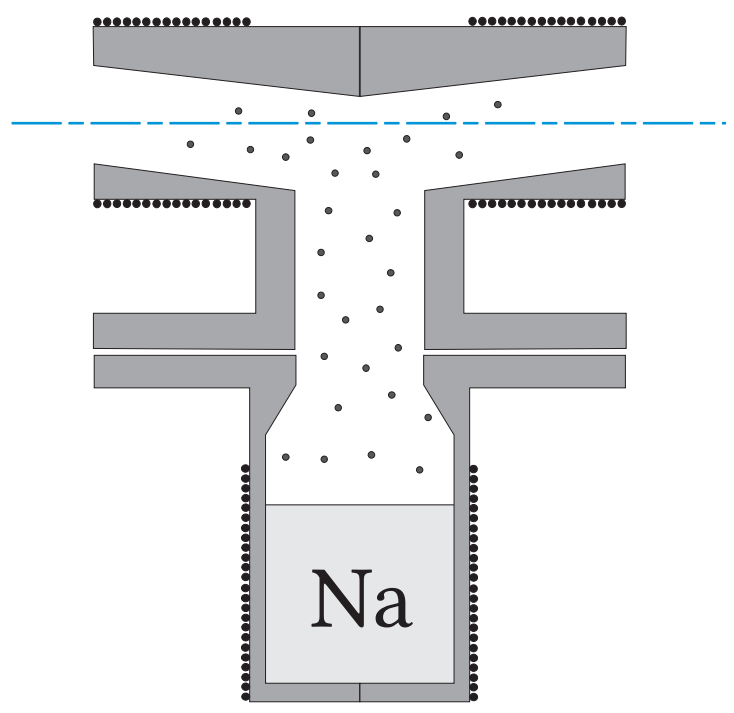

Abbildung 3.2.: Schematischer Aufbau der Pickup-Zelle, entnommen aus [108].

Ventils gerichtet ist. Bei Wiedereinbau der Pickup-Zelle kann nun mit Hilfe eines Spiegels der Durchgang des Laserpointers überprüft und falls nötig die Position der Zelle korrigiert werden. Die Feinjustage erfolgt wieder iterativ über die Aufnahme von Massenspektren.

Die Anzahl $\langle k\rangle$ der in der Pickup-Zelle aufgenommenen Teilchen lässt sich über den Erwartungswert der Poisson-Verteilung [126] abschätzen:

$$
\langle k\rangle=\sigma_{e} n_{g} L
$$

Hierbei ist $n_{g}$ die Teilchendichte in der Gasphase und $\sigma_{e}$ der Einfangsquerschnitt. Dieser unterscheidet sich stark vom geometrischen Querschnitt und ist im Falle der Aufnahme eines Wassermoleküls durch einen Wassercluster bestehend aus 260 Moleküleinheiten um den Faktor 2,5 höher 127. Über den Zusammenhang $n_{g}=p / k_{B} T$ erhält man eine von Temperatur $T$ und Druck $p$ abhängige Gleichung:

$$
\langle k\rangle=\sigma_{e} \frac{p}{k_{B} T} L
$$

Die Wahrscheinlichkeit für die Aufnahme von $\langle k\rangle$ Teilchen ist dabei $P_{k}(p)$ :

$$
P_{k}(p)=\frac{1}{k !}\left(\sigma_{e} n_{g} L\right)^{k} \exp \left(-\sigma_{e} n_{g} L\right)=\frac{1}{k !}\left(\sigma_{e} \frac{p}{k_{B} T} L\right)^{k} \exp \left(-\sigma_{e} \frac{p}{k_{B} T} L\right)
$$




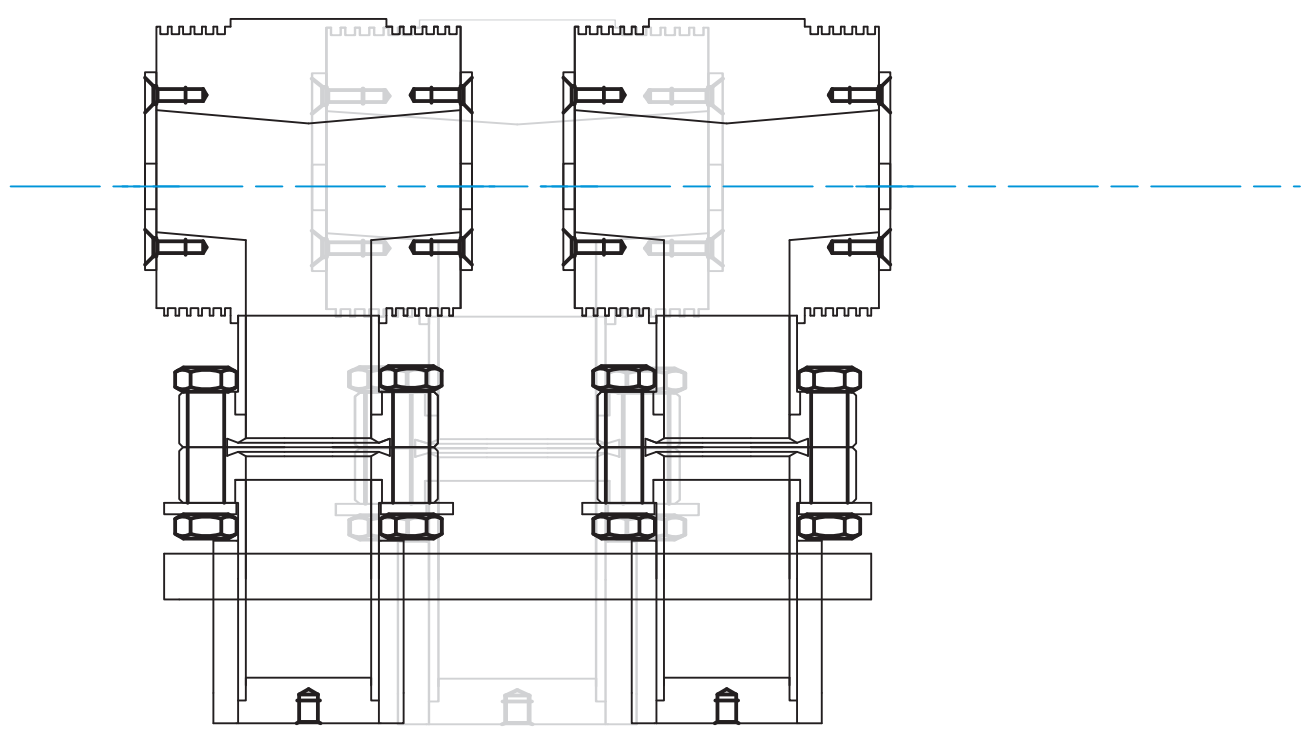

Anordnung mit bis zu zwei kleinen Pickup-Zellen.

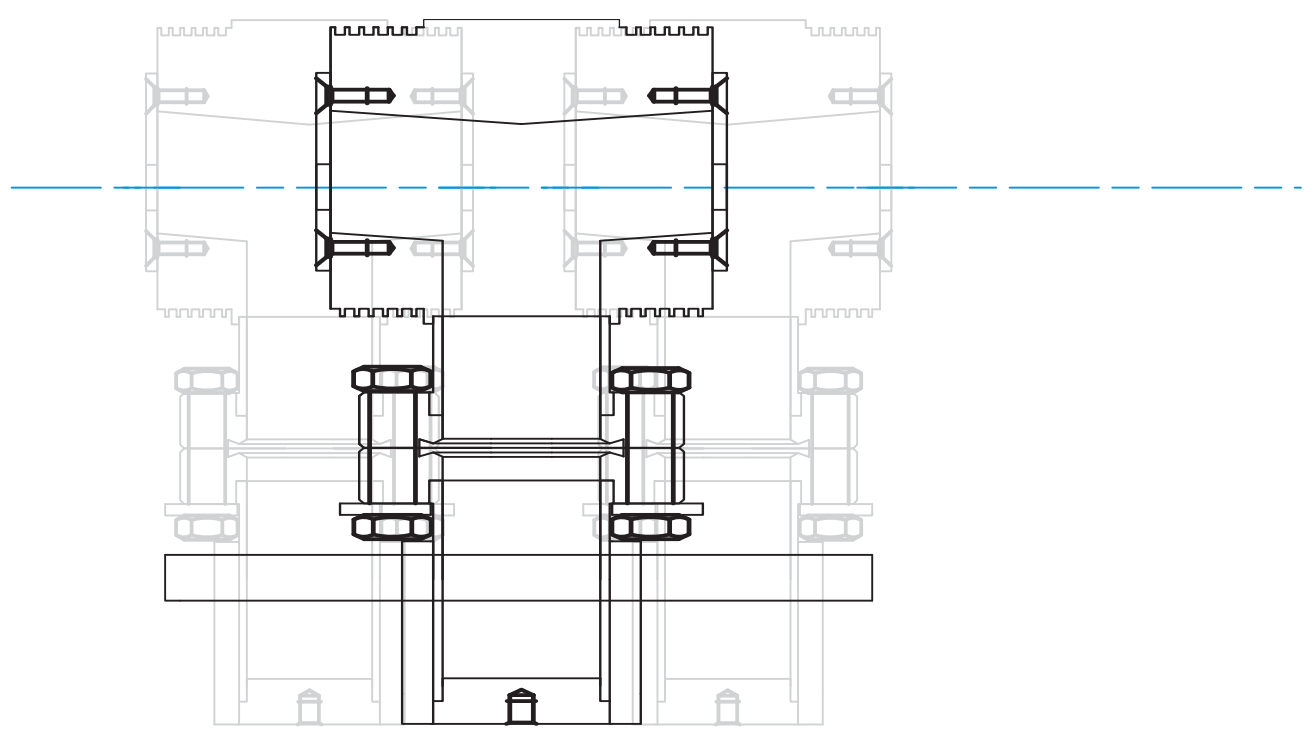

Anordnung mit großer Pickup-Zelle.

Abbildung 3.3.: Unterschiedliche Pickup-Zellenanordnungen, verändert nach den Konstruktionszeichnungen der Doppelpickup-Zelle aus |108. 
Der Dampfdruck des Natriums $p_{\mathrm{Na}}$ kann in einem Temperaturbereich von $454,9 \mathrm{~K}$ bis $1163 \mathrm{~K}$ über die von Rodebush und DeVires 1925 postulierte Antoine Gleichung [128 bestimmt werden:

$$
\log _{10} \frac{p_{\mathrm{Na}}}{\text { Torr }}=12,9605-\left(\frac{5922}{T / \mathrm{K}}\right)-\left(1,6184 \cdot \log _{10} T / \mathrm{K}\right)+8,5874949 \cdot 10^{-5} \mathrm{~T} / \mathrm{K}
$$

Für den Pickup-Prozess werden üblicherweise Drücke unter $10^{-3}$ Torr 129 verwendet. Da die Parameter der Gleichung 3.4 in diesem Bereich mit einer etwas ungenauen Methode bestimmt wurden, haben Buck und Pauly [130 für den Temperaturbereich von $393,5 \mathrm{~K}$ bis $467,9 \mathrm{~K}$ eine exaktere Gleichung entwickelt:

$$
\log _{10} \frac{p_{\mathrm{Na}}}{\text { Torr }}=8,08-\left(\frac{5479}{T / \mathrm{K}}\right)
$$

Für die in dieser Arbeit durchgeführten Messungen wurden das Reservoir der Pickup-Zelle auf $220^{\circ} \mathrm{C}$ und der obere Teil auf $180^{\circ} \mathrm{C}$ erwärmt. Der errechnete Dampfdruck von ungefähr $9,32 \cdot 10^{-4}$ Torr $\left(1,24 \cdot 10^{-6}\right.$ bar $)$ stellt eine obere Grenze für den tatsächlich vorherrschenden Dampfdruck in der Pickup-Zelle dar.

\subsubsection{Detektionskammer}

Die Detektionskammer beinhaltet das Flugzeitmassenspektrometer, das über ein Reflektron verfügt. Ein schematischer Aufbau der Kammer ist in Abbildung 3.4 $\mathrm{zu}$ finden und die zugehörigen Abmessungen sind in Tabelle 3.1 aufgelistet. Die Ionisation erfolgt mit Hilfe von Photonen in dem violett markierten Volumen in der Ionenquelle mit zweistufigem Beschleunigungsfeld. Die Erzeugung der benötigten Photonen wird in Abschnitt 3.2.1 erläutert. Die Massentrennung wird über die Flugzeit realisiert. Eine Multikanalplatte registriert die auftreffenden Ionen. Im Folgenden wird auf die einzelnen Punkte näher eingegangen.

\subsubsection{Ionenquelle mit zweistufigem Beschleunigungsfeld}

Über ein UHV-Ventil gelangen die natriumdotierten Wassercluster in die Detektionskammer. Dort passieren sie eine Stahl-Lochblende, die mit einem Nickelnetz mit einer Transmission von $90 \%$ bespannt ist und an dem die Spannung $U_{\mathrm{a}}$ anliegt. Im violett markierten Volumen in Abbildung 3.4 findet die Ionisation mit 


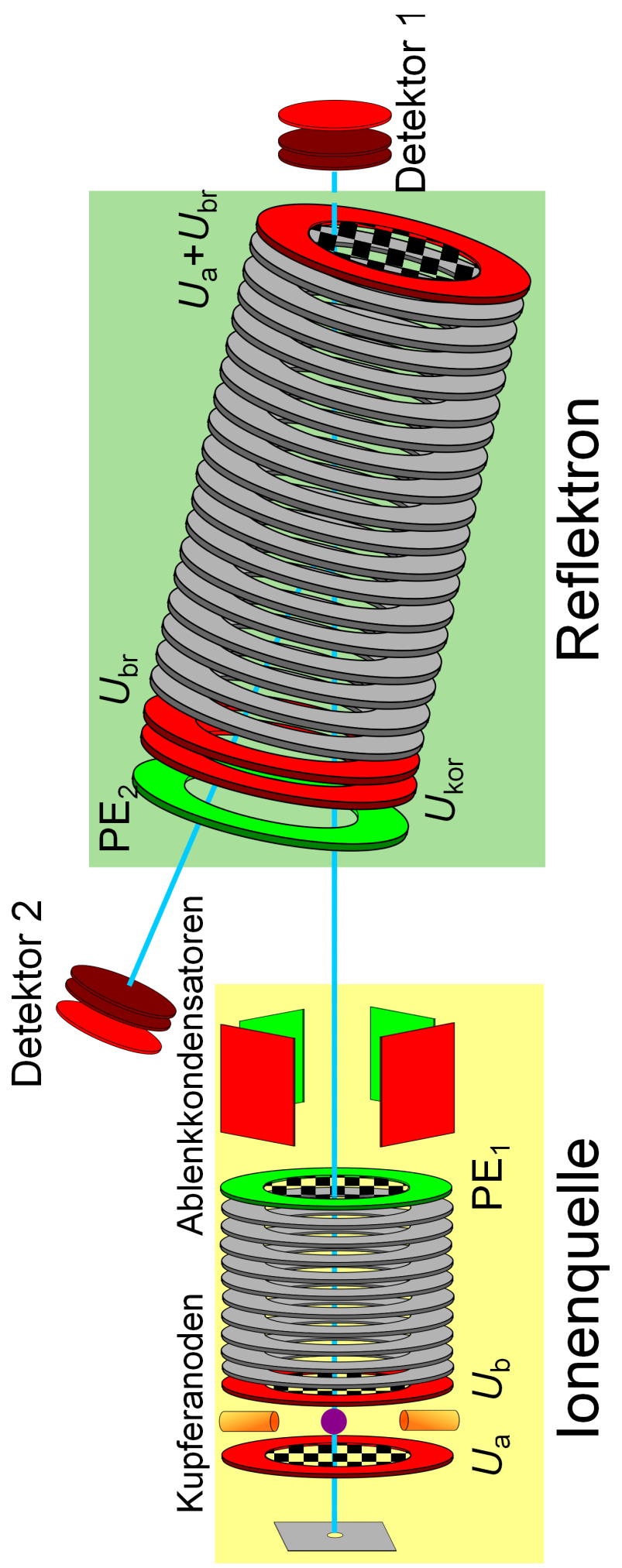

Abbildung 3.4.: Schematischer Aufbau der Detektionskammer. An den roten Platten liegt eine Gleichspannung an, die grünen sind auf Erdpotential. Der violette Kreis repräsentiert das Ionisationsvolumen. Verändert nach $|80|$. 
Tabelle 3.1.: Abmessungen des Reflektron-Flugzeitmassenspektrometers.

\begin{tabular}{l|l|l}
\hline Ionenquelle & Abzugsfeld & $10 \mathrm{~mm}$ \\
& Beschleunigungsfeld & $44 \mathrm{~mm}$ \\
\hline Reflektron & Reflektionsfeld & $216 \mathrm{~mm}$ \\
& Bremsfeld & $6 \mathrm{~mm}$ \\
& Korrekturfeld & $8 \mathrm{~mm}$ \\
\hline Driftstrecke & linear (ohne Reflektron) & $1330 \mathrm{~mm}$ \\
& mit Reflektron & $1820 \mathrm{~mm}$ \\
\hline
\end{tabular}

Photonen aus dem in Abschnitt 3.2.1 beschriebenen Lasersystem statt. Die Elektronen werden über die oben und unten angeordneten, im Durchmesser $3 \mathrm{~mm}$ großen Kupferanoden, an welchen die gleiche Spannung angelegt wird, abgeführt. Da eine Potentialdifferenz zwischen den Lochblenden $U_{\mathrm{a}}$ und $U_{\mathrm{b}}$ anliegt, erfolgt eine Beschleunigung der Kationen in Richtung Reflektron. Eine weitere Beschleunigung erfahren die Cluster durch die Potentialdifferenz zwischen $U_{\mathrm{b}}$ und $\mathrm{PE}_{1}$. Das hierfür benötigte homogene elektrische Feld wird über die zehn grauen Platten erzeugt, die über $1 \mathrm{M} \Omega$ Widerstände mit $U_{\mathrm{b}}$ und $\mathrm{PE}_{1}$ verbunden sind. Die in der Ionenquelle verbauten Platten haben einen Außendurchmesser von $60 \mathrm{~mm}$ und einen Innendurchmesser von $15 \mathrm{~mm}$. Die Platte $U_{\mathrm{a}}$ hat einen geringeren Innendurchmesser von $6 \mathrm{~mm}$ und ist wie die Platten $U_{\mathrm{b}}$ und $\mathrm{PE}_{1}$ mit einem Nickelnetz bespannt. Die Richtung der beschleunigten kationischen Cluster kann über Ablenkkondensatoren ( $40 \mathrm{~mm}$ lang und $25 \mathrm{~mm}$ breit) hinter der Blende $\mathrm{PE}_{1}$ optimiert werden.

Es ist ebenfalls möglich, die Cluster mit Hilfe von Elektronenstoßionisation zu ionisieren 122. Dabei wird ein Laserstrahl auf die untere Kupferanode gerichtet. Die verschiedenen elektrischen Felder werden in Abhängigkeit der Repetitionsrate des Lasers gepulst. Die entstehende kinetische Energie der Elektronen ist abhängig von der Differenz der anfänglich anliegenden Spannungen zwischen oberer und unterer Kupferanode bei Laserbeschuss. Ist an der unteren Anode eine Spannung von $1500 \mathrm{kV}$ und an der oberen $1769 \mathrm{kV}$ angelegt, so entspricht dies einer kinetischen Energie der Elektronen von $50 \pm 8 \mathrm{eV}$ am Ort der Ionisation. Mit einer Anstiegszeit von ungefähr 27 ns relativ zum Laserpuls wird die Spannung der unteren Anode der oberen angeglichen. Die Elektronen treffen im Ionisationsvolumen auf die Cluster. Die Beschleunigungsspannungen $U_{\mathrm{a}}, U_{\mathrm{b}}$ und die Spannung der Ab- 
lenkkondensatoren werden mit einer Anstiegszeit von ungefähr $30 \mathrm{~ns}$ relativ zum Laserpuls bereitgestellt.

Die Ionenquelle ist über M10-Gewindestangen mit der Zwischenkammer verbunden, sodass ihre Position nicht ohne weiteres verändert werden kann. Die Verkabelung erfolgt über einen DN 100 Flansch, der oberhalb der Ionenquelle positioniert ist. Um eine Wartung oder Reparatur durchzuführen, muss der DN 200 Flansch zwischen Detektions- und Zwischenkammer geöffnet werden, was durch das vorhandene Schienensystem erleichtert wird. Für eine erste Diagnose bei Störungen können auch die DN 150 Flansche, die Ein- bzw. Auskoppelfenster für die Lasersysteme besitzen, an der linken und rechten Seite der Detektionskammer ausgebaut werden. Diese befinden sich auf der Höhe der Ionenquelle.

\subsubsection{Reflektron}

Die im Reflektron eintreffenden kationischen Cluster werden mit Hilfe von drei elektrischen Feldern fokussiert, abgebremst und in Richtung des Detektors beschleunigt. Für die Erzeugung der elektrischen Felder werden, wie in der Ionenquelle, Stahl-Lochblenden verwendet, jedoch im Gegensatz zur Ionenquelle, ist lediglich die letzte Platte $U_{\mathrm{a}}+U_{\mathrm{br}}$ mit einem Netz bespannt. Die Blenden weisen einen Außendurchmesser von $180 \mathrm{~mm}$ und einen Innendurchmesser von $100 \mathrm{~mm}$ auf. Es entsteht daher ein inhomogenes elektrisches Feld, das mit zunehmender Plattenanzahl in Richtung $U_{\mathrm{a}}+U_{\mathrm{br}}$ homogener wird (vgl. Abb. 3.5). Die Platte $\mathrm{PE}_{2}$ liegt auf Erdpotential. Das elektrische Feld zwischen dieser und $U_{\text {kor }}$ dient zur besseren Fokussierung des Ionenstrahls. Das nachfolgende Feld (bis $U_{\text {br }}$ ) wird als Bremsfeld bezeichnet. Bis zu diesem Punkt sollen die Ionen zwei Drittel ihrer in der Ionenquelle erhaltenen kinetischen Energie verloren haben. Das letzte Feld wird als Reflektorfeld bezeichnet. Es wird, ähnlich wie das zweite Beschleunigungsfeld der Ionenquelle, über 18 graue Blenden, die über $300 \mathrm{k} \Omega$ Widerstände mit den angrenzenden spannungsversorgten Platten verbunden sind, erzeugt.

Die einzelnen Lochblenden sind auf vier M4-Gewindestangen montiert, die auf einer $5 \mathrm{~mm}$ starken Grundplatte aus Aluminium befestigt sind und werden über Degussitrohre isoliert. Die gesamte Konstruktion ist über vier Federn und drei Metalldornen auf einem DN 200 Blindflansch befestigt, was einen Ausbau für Reparatur- oder Wartungsarbeiten erschwert. Zwei der drei Dornen lassen sich in 
der Länge über Vakuumdurchführungen variieren, so dass das Reflektron horizontal und vertikal verkippt werden kann.

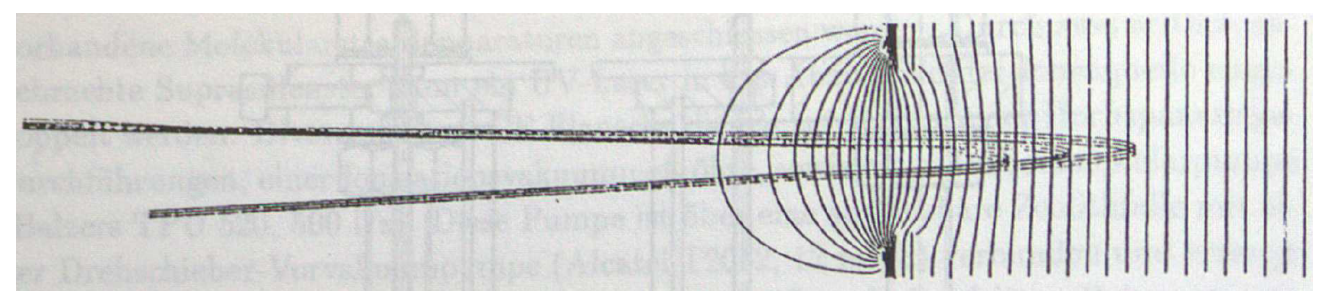

Abbildung 3.5.: Simulation der Ionenbahnen für drei verschiedene Ionenenergien im netzlosen Reflektron nach Risch [131, entnommen aus [121].

\subsubsection{Detektor}

Die als Detektor verwendeten Mikrokanalplatten ( micro channel plate MCP) 132] bestehen aus vielen kleinen Röhren mit einer Größe von $10 \mu \mathrm{m}$. Trifft das Kation auf die Platte, werden Elektronen herausgelöst und durch die leicht geneigte Anordnung der Röhren vervielfacht. Die Signalverstärkung ist dabei abhängig von der angelegten Spannung und kann noch weiter erhöht werden, indem man zwei (chevron) oder drei (z-stack) MCPs hintereinander schaltet. In der Detektionskammer sind zwei Chevron MCPs verbaut. Detektor 1 befindet sich direkt hinter dem Reflektron, um mögliche Defekte im Reflektron abzuklären. Dieser wird im Folgenden als Monitordetektor bezeichnet. Detektor 2 ist oberhalb der Ionenquelle (vgl. Abb. 3.4 angeordnet und wird im regulären Messbetrieb verwendet, um eine möglichst hohe Massenauflösung zu erzielen.

Die eingesetzten MCP-Detektoren haben einen Durchmesser von 25 mm. Der Monitordetektor hatte ursprünglich einen Durchmesser von $33 \mathrm{~mm}$ und stammte von der Firma Hamamatsu (Typ F2223). Dieser ist direkt auf einer Basisplatte verschraubt, die mit dem DN 200 Flansch verbunden ist. In der Mechanikwerkstatt des Instituts wurde eine neue Basisplatte gebaut, auf die ein $25 \mathrm{~mm}$ MCP verschraubt werden kann, sodass in der gesamten Apparatur nun dieser Detektortyp eingesetzt wird. Als Monitordetektor wird ein Chevron MCP der Firma El-Mul (Double MCP; B035VA) verwendet, der Detektor 2 ist von der Firma Tectra (MCP-25-D-S-A). Beide Detektoren unterscheiden sich nur in der Anordnung der Anschlüsse. 
Die Beschaltung des Detektors 2 ist in Abbildung 3.6 gezeigt. Die einzelnen Bauteile befinden sich in der Apparatur. Der Detektor wird durch ein Metallgehäuse nach außen abgeschirmt und enthält vorne eine mit einem Netz bespannte Öffnung, durch die der Ionenstrahl eintreten kann. Um die Detektion großer Cluster zu ermöglichen, ist ein weiteres Kupfernetz mit einer Transmission von 50 \% verbaut. Dieses dient dazu, die Cluster zu fragmentieren und nachzubeschleunigen, da die Nachweisempfindlichkeit nicht von der kinetischen Energie, sondern von der Geschwindigkeit der Teilchen abhängt 122. Die Kondensatoren liefern Ladungsträger nach, um einem kurzzeitigen Zusammenbruch der Detektorspannungen entgegenzuwirken [121. Die angelegten Spannungen betragen für das Fragmentierungsnetz $U_{\mathrm{FR}}=250 \mathrm{~V}$, für die Hochspannung am MCP-Detektor je nach zu erwartendem Signal $U_{\mathrm{HV}}=-1,7$ bis $-1,9 \mathrm{kV}$ und $U_{\mathrm{NV}}=-40 \mathrm{~V}$. Ein Massewiderstand verhindert die statische Aufladung der Anode. Trifft ein Ion auf den MCP, so wird an diesem ein Spannungspeak von $4 \mathrm{mV}$ mit einer Dauer von 2 bis $3 \mathrm{~ns}$ erzeugt, der über einen $50 \Omega$-Widerstand abfällt [46]. Die maximal zulässige Hoch-

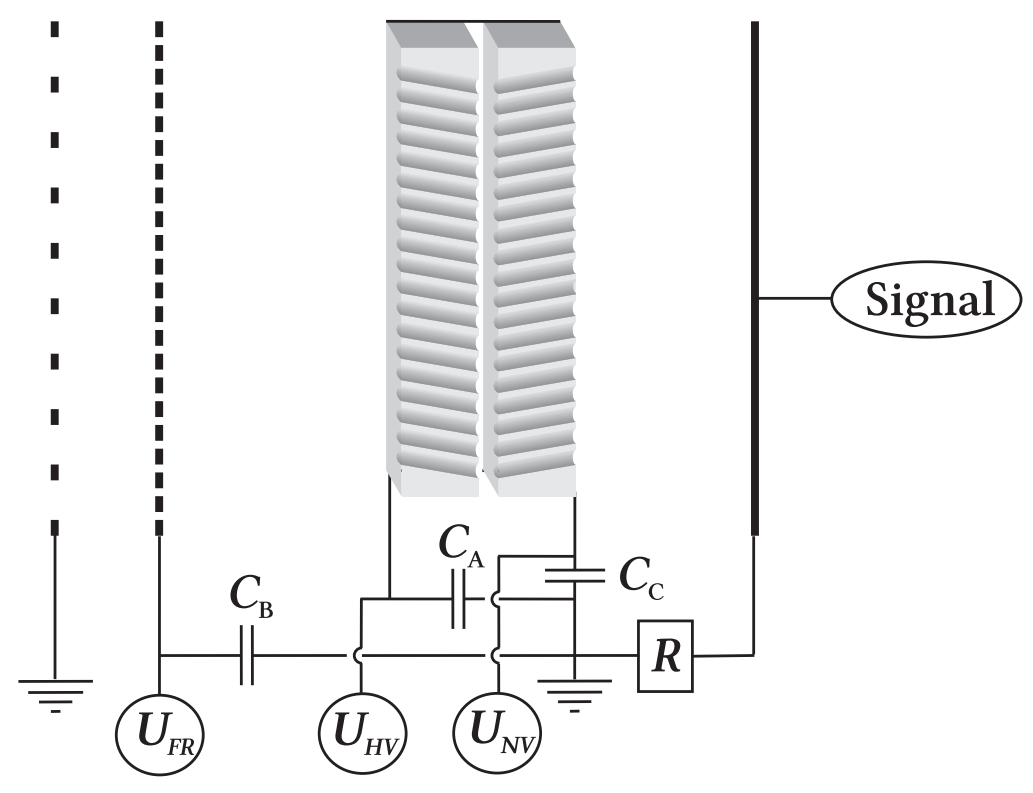

Abbildung 3.6.: Beschaltung des MCP Detektors 2. $\mathrm{C}_{\mathrm{A}}, \mathrm{C}_{\mathrm{B}}$ und $\mathrm{C}_{\mathrm{C}}$ stellen Kondensatoren da. Die Werte der Spannungen $\mathrm{U}_{\mathrm{FR}}, \mathrm{U}_{\mathrm{HV}}$ und $\mathrm{U}_{\mathrm{NV}}$ werden im Text beschrieben. Der Widerstand R beträgt $1 \mathrm{M} \Omega$. Verändert nach |80|.

spannung, die am Chevron MCP angelegt werden darf, beträgt $2 \mathrm{kV}$ und wird zur 
Erhöhung der Lebensdauer nur bei Testmessungen verwendet.

Dektor 1 hat einen veränderten Schaltplan. Er wird nur mit einer geerdeten, netzbespannten Blende gegen die Driftstrecke abgeschirmt. Aus Platzgründen wurde auf das Fragmentierungsnetz verzichtet. Da die Versorgung mit einer Hochspannungsquelle, die über einen Gleichspannungswandler $U_{\mathrm{HV}}$ und $U_{\mathrm{NV}}$ liefert, realisiert wird, kann ebenfalls auf die Kondensatoren verzichtet werden. $U_{\mathrm{HV}}$ ist dabei direkt abhängig von der angelegten Spannung, $U_{\mathrm{NV}}$ wird auf $-40 \mathrm{~V}$ belassen.

\subsubsection{Vakuumsysteme}

Die einzelnen Kammern haben unterschiedliche Anforderungen an das Vakuum und die Saugleistung der verwendeten Pumpen. Eine Auflistung ist in Tabelle 3.2 gegeben.

Da die Cluster in einer kontinuierlichen Expansion erzeugt werden, ist es in der Ofenkammer vor allem wichtig, dass ein großer Durchsatz gewährleistet wird. Dies wird mit Hilfe eines dreistufigen Vakuumpumpsystems bestehend aus Diffusions-, Wälzkolben- und Drehschieberpumpe realisiert. Zusätzlich wurde ein zweiter, als alternativ bezeichneter Pumpstand konstruiert, bestehend aus zwei parallel geschalteten Alcatel RSV350 Wälzkolbenpumpen, die zusammen eine Saugleistung von $375 \mathrm{~m}^{3} \cdot \mathrm{h}^{-1}$ haben. Als Vorpumpe dient hier eine Leybold E150 Sperrschieberpumpe, die Diffusionspumpe bleibt als dritte Stufe erhalten. Beide Pumpstände können auch zusammen betrieben werden, der limitierende Faktor ist dann die Diffusionspumpe. Wird nur der alternative Pumpstand betrieben, ist nicht nur die Saugleistung geringer, sondern auch der Druck im Leerlauf ist, im Vergleich zu dem als regulär bezeichneten Pumpstand, um etwa eine halbe Größenordnung erhöht. Weitere Modifikationen sind in Planung. So soll eine der Alcatel RSV350 Wälzkolbenpumpen durch eine Leybold WS 500 Wälzkolbenpumpe (Saugleistung $500 \mathrm{~m}^{3} \cdot \mathrm{h}^{-1}$ ) ersetzt werden, die dann vor die verbliebene Alcatel-Pumpe geschaltet würde.

Zwischenkammer und Detektionskammer werden über ein zweistufiges Pumpsystem evakuiert. Dieses wurde seit den Arbeiten von Dr. Forck und Dr. Dauster kaum modifiziert [41, 108]. Bei beiden Kammern wird eine Leybold D16B Drehschieberpumpe als Vorpumpe verwendet. In der Zwischenkammer sorgt eine Diffstak 100/300 Öldiffusionspumpe für das Hochvakuum. Die Detektionskammer wird 
mit einer Pfeiffer TMU260 Turbomolekularpumpe evakuiert.

Als Treibmittel für die Diffusionspumpe wird Diffelen normal für die Ofenkammer und Diffelen ultra für die Zwischenkammer verwendet 11 Die Drehschieberund Wälzkolbenpumpen werden mit dem hausinternen Pumpenöl betrieben.

Die Drucküberwachung des Hochvakuums der einzelnen Kammern wird mit Hilfe von Ionisationsvakuummetern durchgeführt. In der Ofenkammer kommt ein Penning-Vakuummeter, in der Zischenkammer und Detektionskammer jeweils ein Bayard-Alpert-Vakuummeter zum Einsatz. Der Druck zwischen den einzelnen Pumpen wird mit Hilfe von Pirani-Manometern überwacht. Diese sind über einen Schaltkasten mit der Stromversorgung der Hochvakuumpumpen verbunden und schalten ab einen Vakuum von $1 \cdot 10^{-1}$ mbar die jeweilige Hochvakuumpumpe ab. Eine weitere Sicherheitsschaltung überwacht den Kühlwasserfluss von Turbo- und Diffusionspumpen.

Tabelle 3.2.: Auflistung der verwendeten Pumpen.

\begin{tabular}{c||c|c|c}
\hline Kammer & Fabrikat & Pumpentyp & Saugleistung \\
\hline \hline Ofenkammer & Leybold D60A & Drehschieber & $60 \mathrm{~m}^{3} \cdot \mathrm{h}^{-1}$ \\
regulär & Balzers WKP500 & Wälzkolben & $500 \mathrm{~m}^{3} \cdot \mathrm{h}^{-1}$ \\
& Leybold DI6000 & Öldiffusion & $6000 \mathrm{~L} \cdot \mathrm{s}^{-1}$ \\
\hline Ofenkammer & Leybold E150 & Sperrschieber & $150 \mathrm{~m}^{3} \cdot \mathrm{h}^{-1}$ \\
alternativ & 2xAlcatel RSV350 & Wälzkolben & $375 \mathrm{~m}^{3} \cdot \mathrm{h}^{-1}$ \\
& Leybold DI6000 & Öldiffusion & $6000 \mathrm{~L} \cdot \mathrm{s}^{-1}$ \\
\hline Zwischenkammer & Leybold D16B & Drehschieber & $16 \mathrm{~m}^{3} \cdot \mathrm{h}^{-1}$ \\
& Diffstak 100/300 & Öldiffusion & $300 \mathrm{~L} \cdot \mathrm{s}^{-1}$ \\
\hline Detektionskammer & Leybold D16B & Drehschieber & $16 \mathrm{~m}{ }^{3} \cdot \mathrm{h}^{-1}$ \\
& Pfeiffer TMU260 & Turbo & $210 \mathrm{~L} \cdot \mathrm{s}^{-1}$ \\
\hline
\end{tabular}

\subsection{Lasersysteme}

Im Kapitel 3.1.3.1 wurde bereits erwähnt, dass die mit Natrium dotierten Cluster mit Hilfe von Photonen ionisiert werden. Die hierfür verwendeten Lasersysteme sollen im Folgenden vorgestellt werden. Dabei kamen zwei unterschiedliche experimentelle Methoden zum Einsatz.

\footnotetext{
${ }^{1}$ Beide Treibmittel stammen von der Firma Oerlikon Leybold Vacuum und werden mittlerweile nicht mehr vertrieben. Das Ersatzprodukt hat die Bezeichnung LVO 500.
} 
Die Teilchen können direkt mit einem UV/Vis-Photon ionisiert werden. Nimmt man nun Massenspektren bei verschiedenen UV/Vis-Wellenlängen auf, erhält man die IE der dotierten Cluster [46,60,61, 108.

Werden die dotierten Cluster zuerst mit einem IR-Photon schwingungsangeregt und $80 \mathrm{~ns}$ später über ein UV/Vis-Photon ionisiert, so erhält man bei gleichbleibender UV/Vis-Wellenlänge und Variation der IR-Wellenlänge ein Schwingungspektrum, das über quantenchemische Rechnungen möglichen Strukturen/Isomeren zugeordnet werden kann $40,42,47$.

\subsubsection{UV/Vis-Lasersystem}

Eine zentrale, im Rahmen dieser Arbeit vorgenommene Modifikation des Experiments ist die Verwendung eines neuen UV/Vis-Lasersystems. Da jedoch fast alle vorgestellten experimentellen Daten noch mit dem vorherigen System aufgenommen wurden, wird dieses bereits in vielen vorherigen Arbeiten beschriebene System 41, 108 auch hier kurz vorgestellt. Detaillierter wird auf das neue System bestehend aus einem Nd:YAG-Pumplaser (Continuum Powerlite ${ }^{T M}$ DLS 9010 Lasers), der wahlweise einen optisch parametrischen Oszillator (im weiteren mit OPO bezeichnet) der Firma Continuum (Panther EX OPO) oder einen Farbstofflaser (Sirah Cobra-Stretch) pumpt, eingegangen.

Die Abbildung 3.7 enthält die Massenspektren, die jeweils mit dem alten bzw. neuen Ionisationslaser erzeugt wurden und zeigen, dass die Photoionisation mit dem neuen Lasersystem beim vorhandenen experimentellen Aufbau möglich ist. Eine direkte Vergleichbarkeit ist aufgrund der zahlreichen experimentellen Umbauten (Veränderungen an der Vakuumkammer und dem Detektor) schwer möglich, jedoch scheint das erhaltene Massensignal bei Ionisation mit dem Powerlite 9010 deutlich schwächer zu sein. Dies könnte an dem inhomogenen Strahlprofil sowie kürzeren Laserpulslängen liegen. Für die Expansion wurden die gleichen Temperaturen verwendet (vgl. Tabelle A.2 auf VI), jedoch ist der Stagnationsdruck für den oberen Fall um 0,5 bar größer.

\subsubsection{Excimerlaser gepumptes UV/Vis-Lasersystem}

Die Erzeugung der durchstimmbaren UV/Vis-Photonen erfolgte mit einem Excimerlaser (Lambda Physik LPX205iCC) gepumpten Farbstofflaser (Lambda Physik 


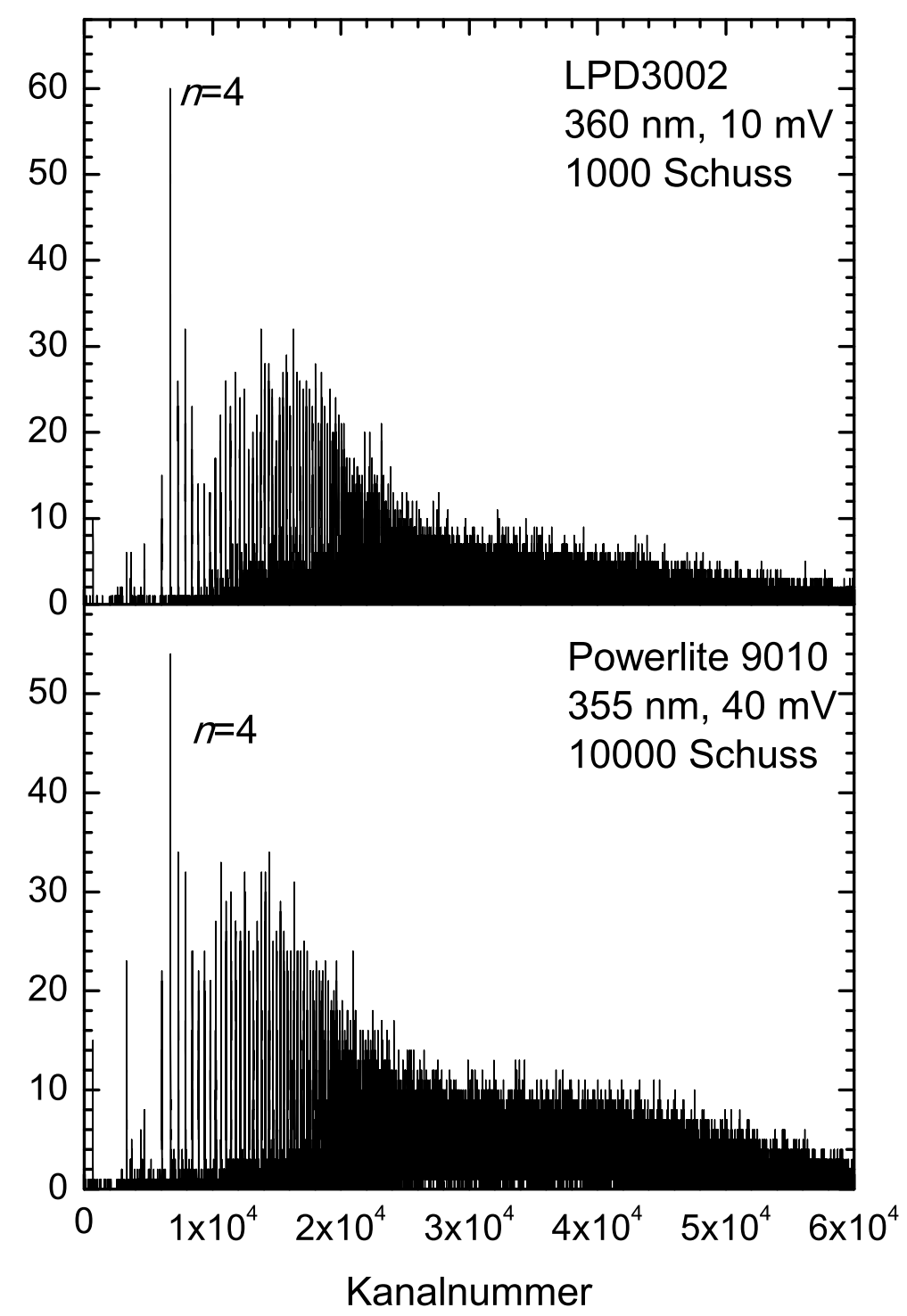

Abbildung 3.7.: Vergleich der nicht normierten Massenspektren bei denen die Ionisation mit dem Excimer-Laser gepumpten UV/Vis-Lasersystem (oben) [02.05.2012, a157] bzw. Powerlite 9010 (unten) [18.07.2013, a054] erfolgte. Der höchste Peak entspricht dem $\mathrm{Na}\left(\mathrm{H}_{2} \mathrm{O}\right)_{4}{ }^{-}$ Cluster. 
LPD3002). Der Excimerlaser wurde dabei mit XeCl als angeregtes Dimer ${ }^{2}$ betrieben und emittierte Licht mit einer Wellenlänge von $308 \mathrm{~nm}$ mit einer Leistung von bis zu 280 mJ pro Puls. Der Laser wurde, wie der in Kapitel 3.2 .2 beschriebene Nd:YAG-Pumplaser, mit einer Repetitionsrate von $10 \mathrm{~Hz}$ betrieben, um den auftretenden Jitter-Effekt zu minimieren. Die FWHM (full width at half maximum) Pulslänge beträgt ca. 20 ns 108. Neben dem optischen Pumpen des Farbstofflasers konnte der Excimerlaser auch direkt in die Apparatur eingekoppelt werden. Die $308 \mathrm{~nm}$ des Excimerlasers wurden über zwei Spiegel in den Farbstofflaser geleitet. Dort wurde ein Teil der Strahlung direkt auf eine $20 \mathrm{~mm}$ lange Küvette gerichtet, welche mit Hilfe eines Gitters die ausgewählte UV/Vis-Wellenlänge erzeugt. Im Weiteren passiert der Laserstrahl über einen Resonatorspiegel erneut die $20 \mathrm{~mm}$ Küvette, die vorher zeitlich versetzt nochmals über die Pumpwellenlänge angeregt wurde, und wird verstärkt. Es findet eine weitere Verstärkung der erzeugten Strahlung über eine $40 \mathrm{~mm}$ lange Küvette statt. Der Aufbau ähnelt dem neuen Farbstofflaser, dessen schematische Aufbauskizze in Abbildung 3.12 auf Seite $55 \mathrm{zu}$ finden ist. Die mögliche Wellenlänge der erzeugten Strahlung ist dabei einerseits abhängig vom verwendeten Gitter, andererseits vom verwendeten Laserfarbstoff. Eine Auflistung der verwendeten Farbstoffe ist in Tabelle 3.3 gegeben. Als Lösungsmittel diente 1,4-Dioxan. Die Pulslänge betrugt 20-30 ns 41,108.

Tabelle 3.3.: Liste der verwendeten Laserfarbstoffe für den Lambda Physik LPD3002 Farbstofflaser.

\begin{tabular}{l|cccc}
\hline Farbstoff & Peak / nm & Wellenlängenbereich / nm & Effizienz & Lösungsmittel \\
\hline \hline DMQ & 360 & $346-377$ & $9 \%$ & 1,4 -Dioxan \\
QUI & 390 & $368-402$ & $11 \%$ & 1,4 -Dioxan \\
PBBO & 396 & $386-420$ & $7 \%$ & 1,4 -Dioxan \\
\hline
\end{tabular}

\subsubsection{Nd:YAG-Pumplaser Powerlite 9010}

Das optische Pumpen der durchstimmbaren UV/Vis-Lasersysteme wird mit Hilfe eines frequenzvervielfachten Nd:YAG-Lasers der Firma Continuum (Powerlite ${ }^{T M}$ DLS 9010) realisiert, der Strahlung mit 1064 nm emittiert. Für den Panther EX OPO (vgl. Abschnitt 3.2.1.3) wird die frequenzverdreifachte Fundamentalwellen-

\footnotetext{
${ }^{2}$ Das Wort Excimer steht dabei für excited dimer
} 
länge $(355 \mathrm{~nm})$ verwendet. Der Cobra-Stretch (vgl. Abschnitt 3.2.1.4) kann wahlweise mit der frequenzverdreifachten oder frequenzverdoppelten Fundamentalwellenlänge $(532 \mathrm{~nm})$ gepumpt werden. Ein schematischer Aufbau ist in Abbildung 3.8 gegeben.

Ein Yttrium-Aluminium-Granat-Kristall $\left(\mathrm{Y}_{3} \mathrm{Al}_{5} \mathrm{O}_{12}\right)$, der mit ca. 0,6 bis $1,4 \%$ $\mathrm{Nd}^{3+}$ Ionen dotiert ist, stellt im Nd:YAG-Festkörperlaser das aktive Medium dar. Die im Powerlite 9010 verbauten Kristalle haben eine Länge von $115 \mathrm{~mm}$ und einen Durchmesser von $6 \mathrm{~mm}$ im Oszillator und $9 \mathrm{~mm}$ in den beiden Verstärkern, zudem sind die Kristalle an den Enden antireflexbeschichtet. Die Anregung der $\mathrm{Nd}^{3+}$ Ionen aus dem Grundzustand findet mit Hilfe von Xenon-Blitzlampen statt. Aus dem angeregten Zustand erfolgt ein strahlungsloser Übergang in das ${ }^{4} \mathrm{~F}_{3 / 2}$-Niveau, aus dem wiederum der Laserübergang mit einem Anteil von $60 \%$ in das ${ }^{4} \mathrm{I}_{11 / 2^{-}}$ Niveau erfolgen kann. Dieser Übergang entspricht $1064 \mathrm{~nm}\left(\right.$ etwa $\left.9400 \mathrm{~cm}^{-1}\right)$. Es finden noch weitere Übergänge in die Niveaus ${ }^{4} \mathrm{I}_{9 / 2},{ }^{4} \mathrm{I}_{13 / 2}$ und ${ }^{4} \mathrm{I}_{13 / 2}$ statt, die mit Hilfe von selektiven Spiegeln für den gewünschten Übergang unterdrückt werden. Als letztes erfolgt dann der strahlungslose Übergang in den Grundzustand. Ein Energieleveldiagramm zu den einzelnen Lasermodi ist in Abbildung 3.9 zu finden. Beim Nd:YAG-Laser handelt es sich, mit Ausnahme des Laserübergangs von ${ }^{4} \mathrm{~F}_{3 / 2}$ $\mathrm{zu}{ }^{4} \mathrm{I}_{9 / 2}$, um ein Vierniveausystem. Das Fluoreszenzspektrum von $\mathrm{Nd}^{3+}$ in YAG bei $300 \mathrm{~K}$ in Abbildung 3.10 soll am Beispiel des genutzten $1064 \mathrm{~nm}$ Übergangs verdeutlichen, dass die oben genannten Niveaus durch thermische Gitterschwingungen verbreitert sind, was Einfluss auf die Linienbreite des Laserlichts hat. Durch den Einsatz eines Injection Seeders, der im Pumplaser Powerlite 8000 verwendet und in Abschnitt 3.2.2.1 näher beschrieben wird, kann eine longitudinale Mode ausgewählt werden.

Um die Intensität des Laserstrahls zu erhöhen, kommen zwei Techniken zum Einsatz. Es werden zwei zusätzliche Laserköpfe mit Blitzlampen eingesetzt, die als Verstärker fungieren. Der Aufbau unterscheidet sich lediglich im Durchmesser der Kristalle. Über induzierte Emission wird die gewünschte Mode verstärkt. Die zweite Technik ist eine Gütemodulation (Q-Switch) des Resonators im Oszillator. Ziel dieser Modulation ist, eine maximale Besetzungsinversion zu erreichen und die Emission über einen kurzen, intensiven Puls abzugeben. Realisiert wird dies mit Hilfe einer Pockels-Zelle, welche einen elektro-optischen Schalter darstellt. Liegt keine Spannung an, ist die Pockels-Zelle transparent. Der Resonator arbeitet inef- 


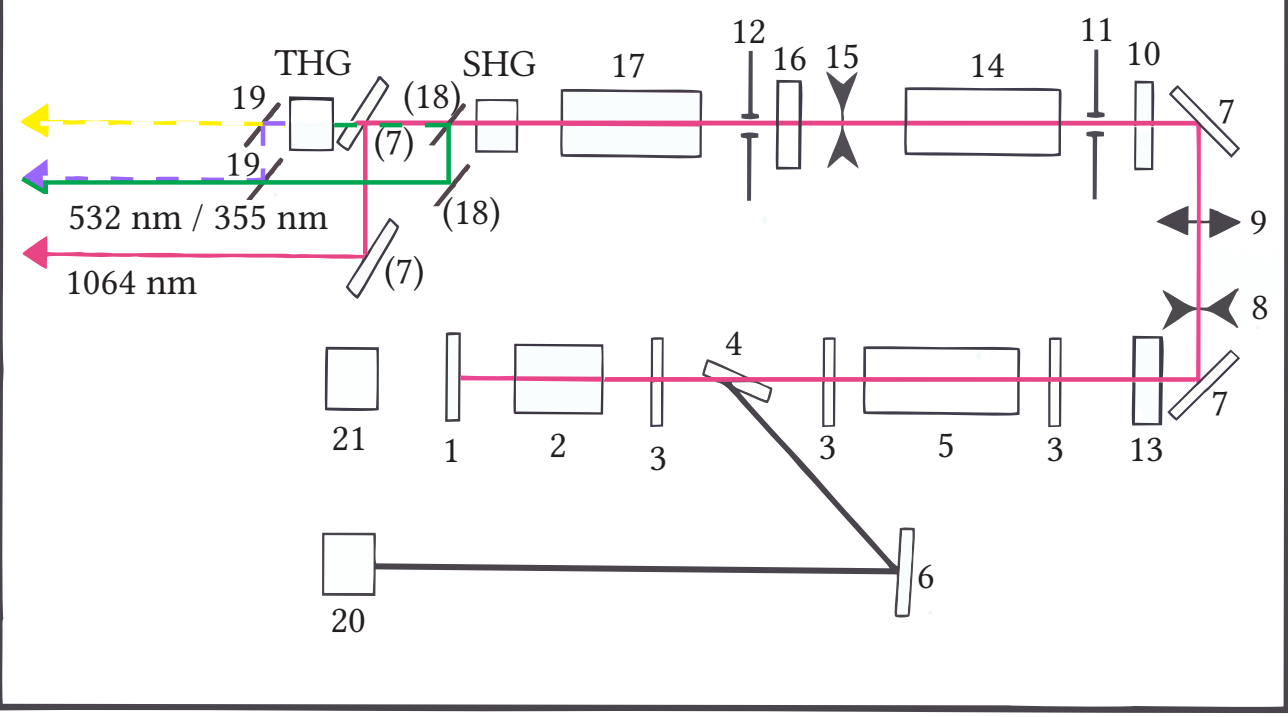

Abbildung 3.8.: Aufbauskizze des Continuum Powerlite ${ }^{T M}$ DLS 9010 Lasers, verändert nach [133. Die optischen Bauteile (7) und (18) werden für den Betrieb mit $532 \mathrm{~nm}$ benötigt.

1. Endspiegel

2. Pockels-Zelle

3. $\lambda / 4$ Platte

4. Dielektrischer Polarisator

5. Laserkopf mit Blitzlampen

6. $30^{\circ}$ Umlenkspiegel (wird nicht verwendet)

7. $45^{\circ}$ Umlenkspiegel $(1064 \mathrm{~nm})$

8. Divergente Linse $(-104 \mathrm{~mm})$

9. Konvergente Linse $(+155 \mathrm{~mm})$

10. $\lambda / 2$-Platte

11. Lochblende $(9,0 \mathrm{~mm})$
12. Lochblende $(9,5 \mathrm{~mm})$

13. Auskoppelspiegel

14. Laserkopf mit Blitzlampen

15. Divergente Linse

16. Rotator

17. Laserkopf mit Blitzlampen

18. Dichroitischer Spiegel $(532 \mathrm{~nm})$

19. Dichroitischer Spiegel $(355 \mathrm{~nm})$

20. Lichtleiter aus dem Seeder (wird nicht verwendet)

21. Lichtleiter zum Seeder (wird nicht verwendet) 


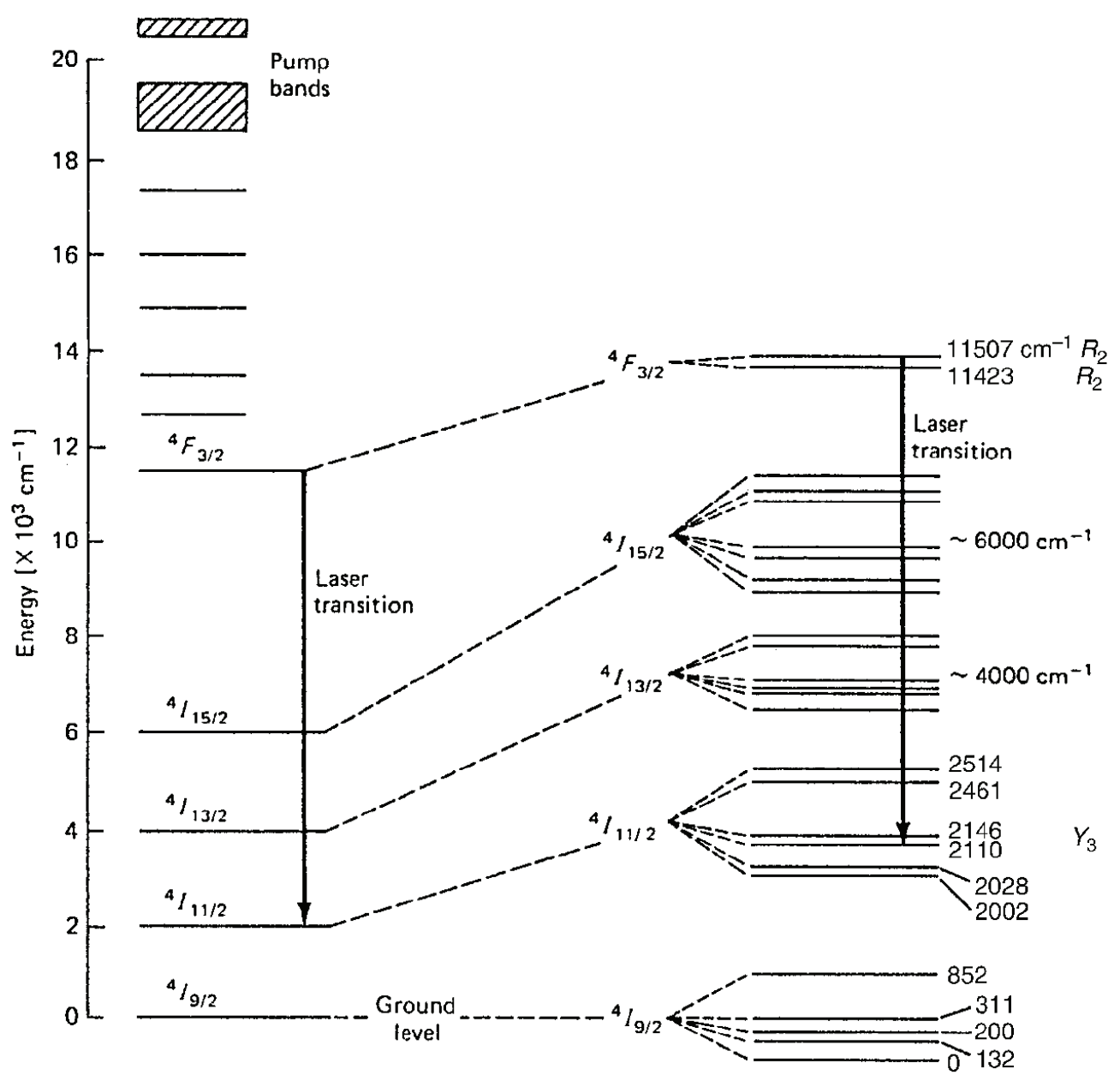

Abbildung 3.9.: Energieleveldiagramm zu Nd $\mathrm{Nd}^{3+}$ als laseraktives Material im Nd:YAG-Laser. Entnommen aus Referenz [134|.

fektiv, da das Licht über einen Polarisator zwischen Pockels-Zelle und Oszillator nach außen hin reflektiert wird. So wird eine hohe Besetzungsinversionsdichte im Oszillator erzeugt. Liegt eine Spannung von 3,6 kV an, ändert sich die Polarisation des Lichtes beim Durchgang durch die Pockels-Zelle. Der Resonator arbeitet effektiv und die stimulierte Emission setzt ein. Eine detailliertere Beschreibung des Bauteils ist in der Dissertation von Dr. Forck zu finden [41].

Für die Erzeugung der frequenzverdreifachten oder frequenzverdoppelten Fundamentalwellenlänge werden Kalium-Dideuterium-Phosphat-Kristalle $\left(\mathrm{KD}_{2} \mathrm{PO}_{4}\right)$ eingesetzt. Das Licht mit der Wellenlänge $532 \mathrm{~nm}$ wird dabei durch Frequenzver- 


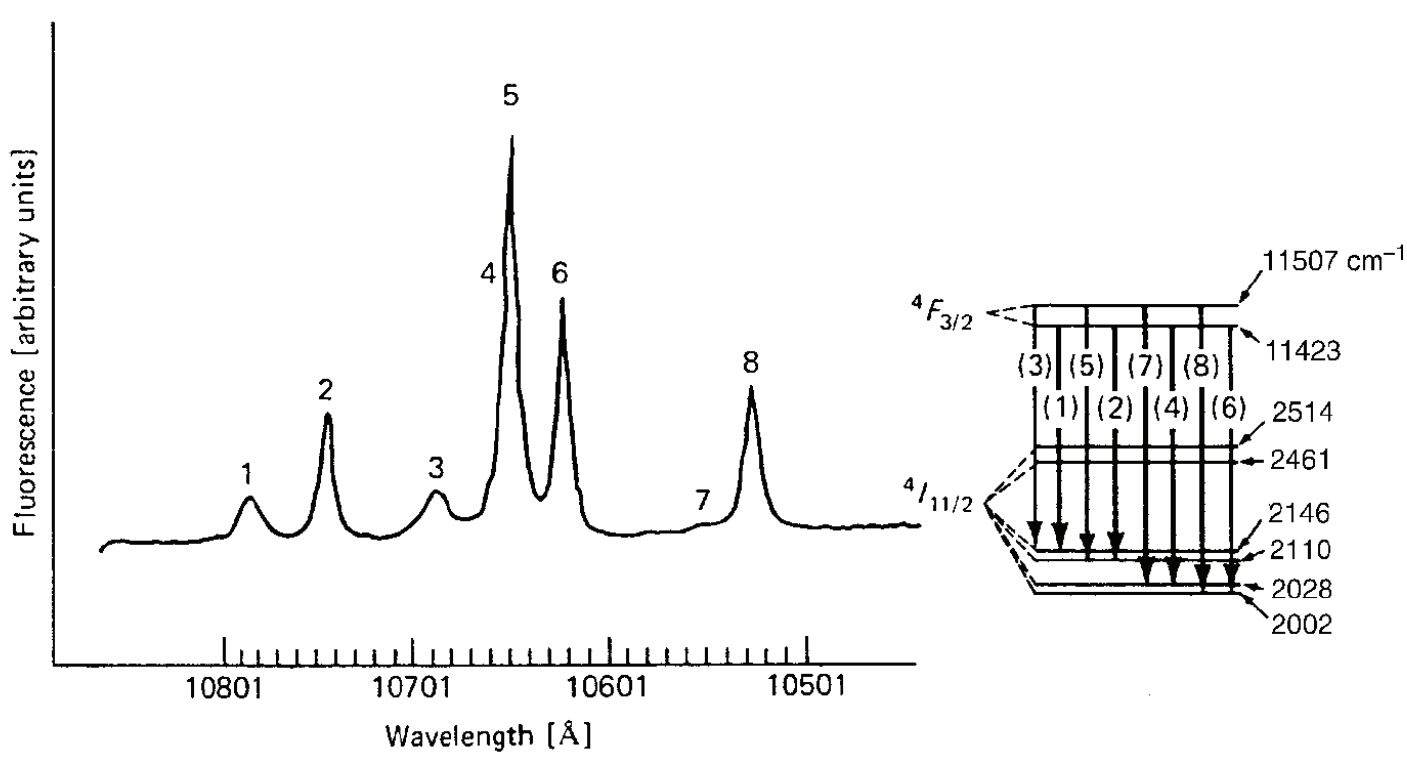

Abbildung 3.10.: Fluoreszenzspektrum von $\mathrm{Nd}^{3+}$ in YAG bei $300 \mathrm{~K}$. Entnommen aus Referenz [134.

dopplung generiert und kann je nach Kristall-Typ (DS-1 SHG 1 oder DS SHG 2) in vertikaler oder horizontaler Polarisation erzeugt werden. Die Polarisation der Fundamentalen muss vorher lediglich über eine $\lambda / 2$ Platte vor den beiden Verstärkern angepasst werden. Die dritte Harmonische resultiert aus einer Summenfrequenzmischung der vorher erzeugten $532 \mathrm{~nm}$ mit der Fundamentalen des YAGs. Die $355 \mathrm{~nm}$ werden dabei mit einer horizontalen Polarisation in einem $\mathrm{KD}_{2} \mathrm{PO}_{4}{ }^{-}$ Kristall Typ 2 (TS THG) erzeugt. Hierfür müssen die zweite Harmonische vertikal (DS-1 SHG 1) und die $1064 \mathrm{~nm}$ horizontal polarisiert sein. Da der Nd:YAG-Laser wahlweise einen auf Strahlprofil und Strahlengang empfindlichen OPO oder einen Farbstofflaser pumpen soll, wurde beim Auskoppeln der einzelnen Wellenlängen darauf geachtet, dass die optische Achse der $355 \mathrm{~nm}$ erhalten bleibt (gestrichelte Linien in Abb. 3.8). Wird die frequenzverdoppelte Fundamentalwellenlänge zum Pumpen des Farbstofflasers benötigt, so geschieht die Auskopplung über zwei dichroitische Spiegel ((7) und (18)), die auf einer optischen Platte installiert sind, die in Strahlrichtung vor dem THG installiert wird (durchgezogene Linien in Abb. 3.8). Der erste Spiegel (18) koppelt die frequenzverdoppelte Fundamentale, der zweite (7) die Fundamentale aus. Es ist damit nicht nötig, die einzelnen Kristalle auszubauen, sodass diese die gesamte Zeit über temperiert werden können, was 
deren Lebenszeit vergrößert.

\subsubsection{Panther EX OPO}

Eine Möglichkeit der Erzeugung von durchstimmbaren Licht im UV/Vis-Bereich stellt der Panther EX OPO der Firma Continuum dar. Er nutzt dabei die frequenzverdreifachte Fundamentalwellenlänge des Nd:YAGs um Licht im Wellenlängenbereich zwischen $410 \mathrm{~nm}$ und $2550 \mathrm{~nm}$ zu erzeugen. Die Linienbreite beträgt ohne den Einsatz eines Seeders dabei je nach Wellenlängenbereich zwischen $4 \mathrm{~cm}^{-1}$ und $6 \mathrm{~cm}^{-1}$. Über ein Verdopplungsmodul bestehend aus zwei Kristallen kann Licht im UV-Bereich zwischen $205 \mathrm{~nm}$ und $410 \mathrm{~nm}$ erzeugt werden. Ein schematischer Aufbau ist in Abbildung 3.11 gegeben. Die Beschreibung der einzelnen Bauteile erfolgt in Tabelle 3.4. Der Panther EX OPO ist über eine optische Platte mit dem Powerlite 9010 verbunden, sodass die $355 \mathrm{~nm}$ direkt in den Laser eingekoppelt werden. Über vier Dichroitische Spiegel (1,3,4,7), einen Abschwächer (5), sowie eine konvexe (6) und eine konkave Linse (8.1), die den Kollimator bilden, gelangt das monochromatische Licht in den Oszillator. Der interne Abschwächer besteht aus einer $\lambda / 2$ Platte und einem Polarisator. Die Funktionsweise kann mit Hilfe eines herausnehmbaren dichroitischen Spiegels in der Position 17.1 oder $17.2 \mathrm{am}$ Ausgang O4 überprüft werden. Dieser Ausgang wird ebenfalls für die direkte Einkoppelung der $355 \mathrm{~nm}$ aus dem Powerlite 9010 genutzt. Im Oszillator (8) bilden die Spiegel 8.2 und 8.4, welche durchgängig für die signal- und idler-Wellenlängen sind, den optischen Resonator für die dritte harmonische des Nd:YAG Lasers. Beim Durchgang durch den BBO-Kristall (8.4) werden signal und idler erzeugt und teilweise durch den Spiegel 8.4 wieder zurück in den Kristall reflektiert. Nach dem zweiten Durchgang durch den Kristall wird der idler über die $\lambda / 2$-Platte nullter Ordnung (8.6a) und das keilförmige Porro-Prisma zurückreflektiert und trifft dann in Spiegel 8.2 auf die $355 \mathrm{~nm}$ des nächsten Pumppulses und wirkt somit als parametrischer Verstärker. Die signal-Wellenlänge wird im Filter 8.5 absorbiert. Damit findet die Resonanz nur mit Hilfe der idler-Wellenlänge statt, was zu einer höheren Stabilität, einem besseren Strahlprofil und zu einer geringeren Linienbreite als die Resonanz mit signal und idler im OPO führt 135. Aufgrund der Beziehung der drei Photonen pump, signal und idler wird die signal-Wellenlänge auch bei jedem idler-Durchgang durch den Kristall gebildet. Ein Kompensator-Kristall 
3.2. Lasersysteme

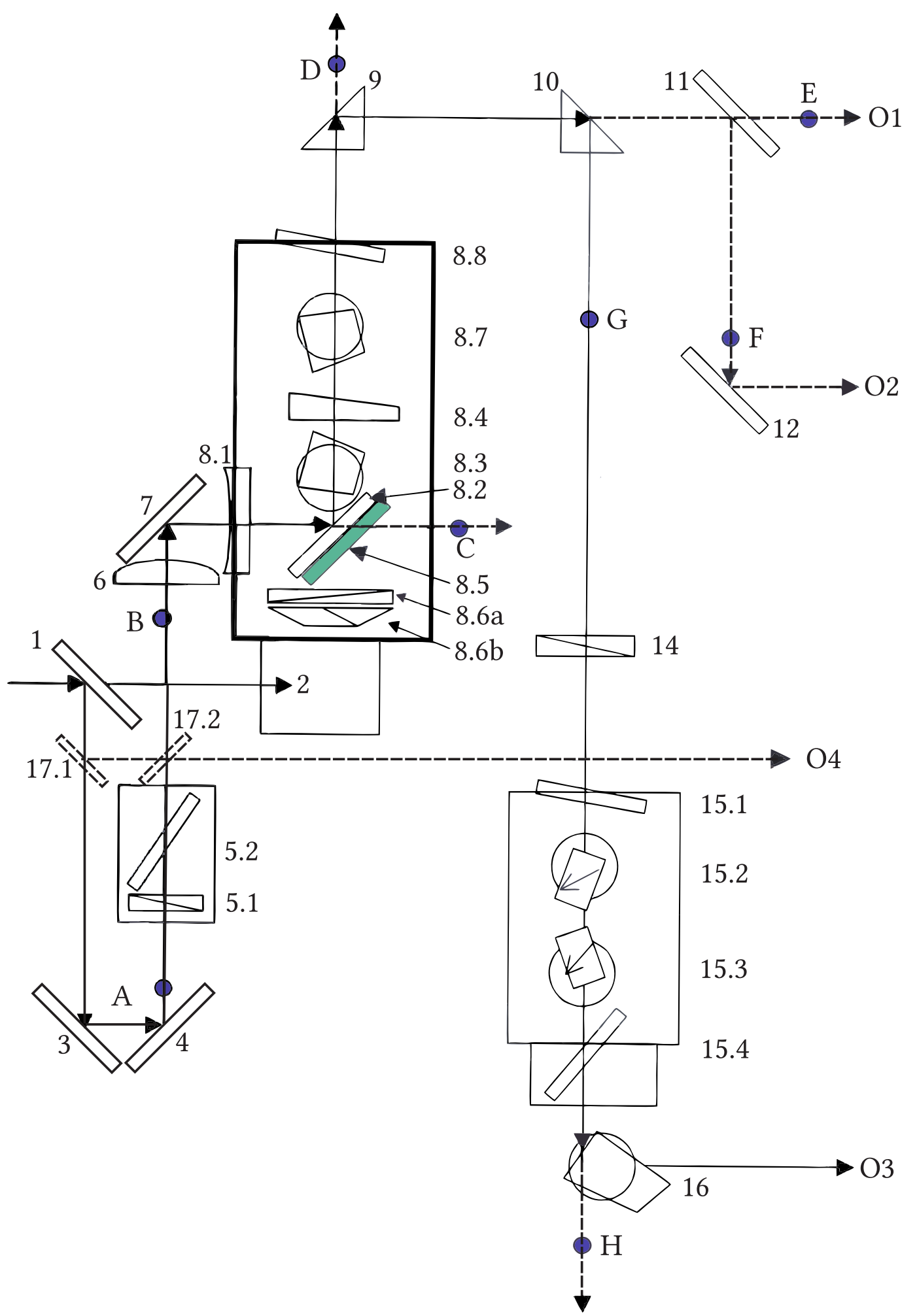

Abbildung 3.11.: Aufbauskizze des Continuum Panther EX OPO II [135]. 
Tabelle 3.4.: Erläuterungen zu Abbildung 3.11 .

Nr. Beschreibung

$\begin{aligned} \text { Nr. } & \text { Beschreibung } \\ 1 & \text { Dichroitischer Spiegel }(355 \mathrm{~nm}) \\ 2 & \text { Strahlfänger } \\ 3 & \text { Dichroitischer Spiegel }(355 \mathrm{~nm}) \\ 4 & \text { Dichroitischer Spiegel }(355 \mathrm{~nm}) \\ 5.1 & \lambda / 2 \text { Platte, antireflexbeschichtet }(355 \mathrm{~nm}) \\ 5.2 & \text { Dielektrischer Polarisator }(355 \mathrm{~nm}) \\ 6 & \text { Konvexe Linse, antireflexbeschichtet }(355 \mathrm{~nm}) \\ 7 & \text { Dichroitischer Spiegel }(355 \mathrm{~nm}) \\ 8.1 & \text { Konkave Linse, antireflexbeschichtet }(355 \mathrm{~nm}) \\ 8.2 & \text { hochrefelektierender Spiegel }(355 \mathrm{~nm}) \\ 8.3 & \text { BBO Kristall Typ II } \\ 8.4 & \text { hochrefelektierender Spiegel keilförmig } \\ 8.5 & \text { Filter für sichtbares Licht } \\ 8.6 \mathrm{a} & \lambda / 2 \text {-Platte nullter Ordnung } \\ 8.6 \mathrm{~b} & \text { Keilförmiges Porro-Prisma } \\ 8.7 & \text { Kompensator Kristall } \\ 8.8 & \text { Breitband Fenster, antireflexbeschichtet } \\ 9 \& 10 & \text { Breitband 90 Prisma, antireflexbeschichtet } \\ 11 \& 12 & \text { Dichroitische breitband Spiegel } \\ 14 & \text { Breitband } \lambda / 2 \text { Platte nullter Ordnung } 800 \mathrm{~nm}, \text { antireflexbeschichtet } \\ 15.1 & \text { Breitband Fenster, antireflexbeschichtet } \\ 15.2 & \text { Verdopplungskristall BBO Type I } \\ 15.3 & \text { Verdopplungskristall BBO Type I } \\ 15.4 & \text { Kalziumfluorid Fenster } \\ 16 & \text { Pellin-Broca Prisma } \\ 17.2 & \text { Dichroitischer Spiegel }(355 \text { nm), herausnehmbar }\end{aligned}$

(8.7) gleicht den Strahlversatz aus, bevor das Wellenpaar den Oszillator über das Auskoppelfenster (8.8) verlässt. Ein Prisma (9) leitet den Strahl zu den möglichen Ausgängen, wo entweder über zwei dichroitische Spiegel (11 und 12) signal (Ausgang O2) und idler (Ausgang O1) getrennt werden oder über ein weiteres Prisma (10) in Richtung der Verdopplungseinheit (15) geleitet werden. Dieses besteht aus zwei BBO-Kristallen (15.2 und 15.3), die sich in ihrer Orientierung unterscheiden. Damit fungieren sie in unterschiedlichen Wellenlängenbereichen für vertikal polarisiertes Licht (vgl. Tabelle 3.5) als Frequenzverdoppler oder als Kompensator. Für die Verdopplung des idlers muss eine $\lambda / 2$ Platte (14) für die Änderung der 
Tabelle 3.5.: Wellenlängenspezifikationen der im Panther EX OPO verbauten $\beta$ Bariumborat Kristalle zum Genieren der zweiten Harmonischen aus signal und idler.

\begin{tabular}{l|c|c}
\hline Nr. bezogen auf Abb. 3.11 & 15.2 & 15.3 \\
\hline Eingangswellenlänge $/ \mathrm{nm}$ & $520-710($ signal $), 710-840($ idler $)$ & $430-520$ \\
Ausgangswellenlänge $/ \mathrm{nm}$ & $260-355$ (signal), $355-420($ idler $)$ & $215-260$ \\
\hline
\end{tabular}

Tabelle 3.6.: Kenndaten des Continuum Panther Ex OPO.

\begin{tabular}{c|c|c}
\hline Spezifikation & Wert & Kommentar \\
\hline Bereich & $205-2550 \mathrm{~nm}$ & davon $410-710 \mathrm{~nm}$ signal \\
Pumpstrahl & $400 \mathrm{~mJ}$ bei $355 \mathrm{~nm}$ & horizontal polarisiert \\
Pulsenergie & $7 \mathrm{~mJ} \pm 2 \mathrm{~mJ}$ & $205-410 \mathrm{~nm}$ \\
& $25 \mathrm{~mJ} \pm 10 \mathrm{~mJ}$ & $410-610 \mathrm{~nm}$ \\
& $60 \mathrm{~mJ} \pm 20 \mathrm{~mJ}$ & $610-700 \mathrm{~nm}$ \\
Repetitionsrate & $15 \mathrm{~mJ} \pm 5 \mathrm{~mJ}$ & $700-2550 \mathrm{~nm}$ \\
& maximal $10 \mathrm{~Hz}$ &
\end{tabular}

Polarisation hineingedreht werden. Die Trennung der frequensverdoppelten Wellenlängen signal und idler erfolgt mit Hilfe eines Pellin-Broca-Prismas (16) im Ausgang O3. Die Leistungsdaten des Panther EX OPO sind in Tabelle 3.6 enthalten.

Die Steuerung des Lasersystems erfolgt mit Hilfe eines auf WinXP-basierenden Programms, das die Rotation der Kristalle für die vorgegebene Wellenlänge anpasst. Hierfür werden Schrittmotoren verwendet, deren Position für jede einzelne Wellenlänge gespeichert ist. Die Wellenlänge wird mit Hilfe eines Spektrometers der Firma Thorlabs (CCS 100) überprüft, da eine Dejustage aufgrund der starken Winkelabhängigkeit der Wellenlänge von der Kristallposition des Öfteren auftritt. Dieses weist im Wellenlängenbereich von 350 bis $700 \mathrm{~nm}$ eine Halbwertsbreite von weniger als $0,5 \mathrm{~nm}$ auf.

\subsubsection{Farbstofflaser Sirah Cobra-Stretch}

Die frequenzverdoppelte oder -verdreifachte Fundamentalwellenlänge des Powerlite 9010 gelangt über einen Spiegel, der auf einer optischen Platte zwischen Panther EX OPO und Pumplaser verbaut ist, in den Farbstofflaser Cobra-Stretch der Firma Sirah. Falls der Laser mit $355 \mathrm{~nm}$ optisch gepumpt werden soll, muss die 
Polarisation mit Hilfe einer $\lambda / 2$-Platte von horizontal in vertikal geändert werden. Ebenfalls ist ein Austausch der Spiegel vonnöten, da diese speziell für die Pumpwellenlänge beschichtet sind. Strahlteiler und Prisma können für beide Wellenlängen verwendet werden. Die $532 \mathrm{~nm}$ besitzen im Falle der Erzeugung mit dem DS-1 SHG 1 Modul im Powerlite 9010 die richtige Polarisation. Der Aufbau des Cobra-Stretch ist dem des Lambda Physics LPD3002 Farbstofflaser ähnlich und in Abbildung 3.12 dargestellt. Erfolgt das optische Pumpen mit $532 \mathrm{~nm}$, so kann die erzeugte Laserstrahlung mit Hilfe eines $\beta$-Bariumborat-Kristalls verdoppelt werden.

Im Farbstofflaser ist ein starres, $60 \mathrm{~mm}$ langes Gitter mit einer Gitterkonstante von 2400 Linien (Stegen) $]^{3}$ pro mm verbaut, welches mit Hilfe eines beschichteten Aluminiumspiegels die gewünschte Wellenlänge einstellt. Optional kann dieser Spiegel durch ein zweites Gitter (wie in der Aufbauskizze gezeigt) ersetzt werden, um die spektrale Auflösung bei geringen Leistungseinbußen zu verbessern. Die Spezifikationen des Gitters bezogen auf das optische Pumpen von Rhodamin 6G mit $532 \mathrm{~nm}$ bei einer eingestellten Wellenlänge von $570 \mathrm{~nm}$ sind in Tabelle 3.7 abgebildet.

Eine $20 \mathrm{~mm}$ lange Küvette dient als Oszillator und Vorverstärker. Im Hauptver-

Tabelle 3.7.: Spezifikationen des Sirah Cobra-Stretch, entnommen aus 136. Die Effizienz ist bezogen auf den Betrieb mit Rhodamin $6 \mathrm{G}$ bei einer Wellenlänge von $570 \mathrm{~nm}$.

\begin{tabular}{c|c|c|c}
\hline verbautes Gitter & Frequenzbereich & Linienbreite & Effizienz \\
\hline 2400 Linien $/ \mathrm{mm}$ & $370 \mathrm{~nm}-760 \mathrm{~nm}$ & $0.08 \mathrm{~cm}^{-1}(2,7 \mathrm{pm})$ & $30 \%$ \\
\hline
\end{tabular}

stärker wird eine Kapillare mit $6 \mathrm{~mm}$ Durchmesser verwendet. Diese erlaubt es, den Laser mit bis zu 1,5 J Pumpenergie zu betreiben und verbessert das Strahlprofil für die Erzeugung der zweiten Harmonischen. Der Einsatz einer $40 \mathrm{~mm}$ langen, rechteckigen Küvette ist ebenfalls möglich. Hierfür ist eine Änderung des Teleskops zur stärkeren Fokussierung des Laserstrahls nötig. Zusätzlich muss die Pumpenergie reduziert und die Konzentration des verwendeten Laserfarbstoffes angepasst werden. Die Kapillare benötigt eine Verdünnung im Verhältnis zum Oszillator von $1 \mathrm{zu} 6$ bis $1 \mathrm{zu} 8$ je nach Farbstoff, die rechteckige Küvette benötigt $1 \mathrm{zu} 3$.

\footnotetext{
${ }^{3}$ Die Gitterkonstante bezieht sich hier auf die Anzahl der Abfolge von Stegen und Furchen in einem $\mathrm{mm}$.
} 


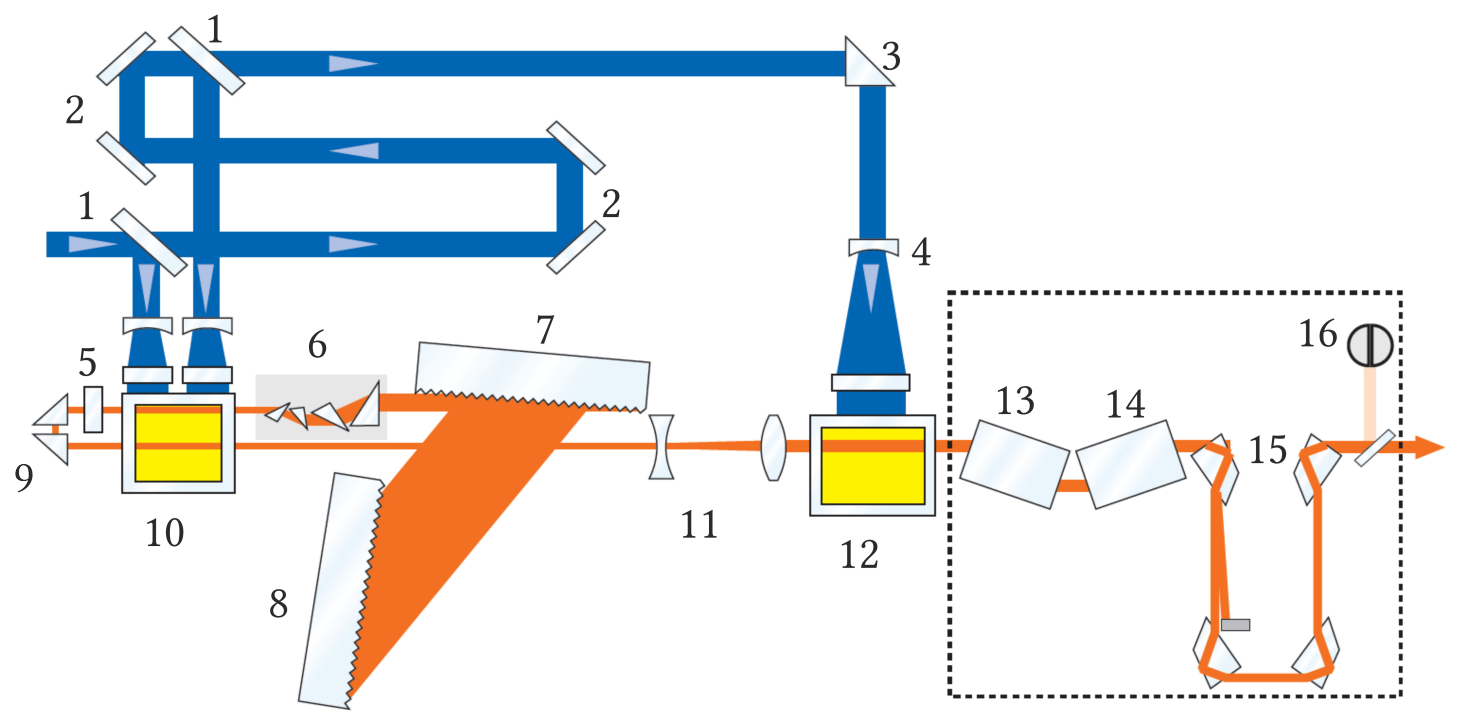

Abbildung 3.12.: Aufbauskizze des Sirah Cobra-Stretch mit Verdopplungseinheit verändert nach [136. Der Pumpstrahl (355 oder $532 \mathrm{~nm}$ ) ist in blau dargestellt, der erzeugte Laserstrahl in orange.

1. Strahlteiler

2. Dichroitische Spiegel (355 nm oder $532 \mathrm{~nm})$

3. Prisma beschichtet (355 $\mathrm{nm}$ und $532 \mathrm{~nm})$

4. Zylinderlinse

5. Auskoppelspiegel

6. Strahlaufweiter

7. Gitter

8. beschichteter Aluminiumspiegel oder Gitter
9. Breitbandspiegel

10. $20 \mathrm{~mm}$-Küvette

11. Teleskop

12. rechteckige $40 \mathrm{~mm}$-Küvette oder 6 mm-Kapillare

13. Verdopplungskristall

14. Kompensator

15. Pellin-Broca Prismen

16. Pyroelektrischer Sensor

Die Frequenzverdopplung der erzeugten Laserstrahlung geschieht mittels eines $\beta$-Bariumborat-Kristalls, der einen Wellenlängenbereich von $280 \mathrm{~nm}-450 \mathrm{~nm}$ für die verdoppelte Ausgangswellenlänge abdeckt. Optional können auch andere $\beta$-Bariumborat-Kristalle verbaut werden, die die Durchstimmbarkeit der erzeugten 
Laserstrahlung bis $215 \mathrm{~nm}$ beim optischen Pumpen des Farbstofflasers mit $355 \mathrm{~nm}$ ermöglichen. Jedoch sind hier die Leistungsspezifikationen des Panther EX OPO Systems besser. Ein Kompensatorkristall gleicht den entstehenden Strahlversatz aus. Über vier Pellin-Broca-Prismen werden UV-Strahlung und sichtbares Licht getrennt.

Die Wellenlängeneinstellung des Cobra-Stretch erfolgt über die mitgelieferte Konsole. Zusätzlich verfügt der Laser über eine USB-Schnittelle. Dies ist vor allem bei der Verwendung der SHG-Option von Vorteil, da so direkt die Kristallposition optimiert werden kann. In der Verdopplungseinheit ist ein pyroelektrischer Sensor verbaut. Am Ende des Strahlengangs wird über einen Strahlteiler 1\% des Lichts auf diesen Sensor reflektiert, der aus zwei Hälften besteht. Das System versucht beim Scannen dann mit Hilfe der Kristallpositionen von Verdopplungskristall und Kompensator den Energiebeitrag auf beiden Hälften anzugleichen.

Die Effizienz des Farbstofflasers und die damit verbundenen Leistungsdaten sind abhängig vom verwendeten Laserfarbstoff. Die Tabelle 3.8 liefert einen groben Überblick über die Leistungsdaten. Die Effizienz der mit $355 \mathrm{~nm}$ gepumpten Farbstoffe liegt bei etwa $15 \% \pm 5 \%$. Sie decken dabei einen Wellenlängenbereich von $372 \mathrm{~nm}$ bis $610 \mathrm{~nm}$ ab. Die mit $532 \mathrm{~nm}$ gepumpten Farbstoffe weisen eine höhere Effizienz auf. Zudem hat der Pumplaser eine höhere Energie. Es wird mit dem verwendeten Gitter ein spektraler Bereich zwischen $540 \mathrm{~nm}$ und $760 \mathrm{~nm}$ mit einer Effizienz von $25 \% \pm 5 \%$ abgedeckt.

Tabelle 3.8.: Kenndaten des Sirah Cobra-Stretch mit Verdopplungseinheit. Die kursiven Einträge beziehen sich auf das optische Pumpen mit 355 $\mathrm{nm}$.

\begin{tabular}{c|c|c}
\hline Spezifikation & Wert & Kommentar \\
\hline Bereich & $280-760 \mathrm{~nm}$ & \\
Pumpstrahl & $400 \mathrm{~mJ}$ bei $355 \mathrm{~nm}$ & vertikal polarisiert \\
Pumpstrahl & $600 \mathrm{~mJ}$ bei $532 \mathrm{~nm}$ & vertikal polarisiert \\
Pulsenergie & $25 \mathrm{~mJ} \pm 10 \mathrm{~mJ}$ & $280-380 \mathrm{~nm}$ \\
& $60 \mathrm{~mJ} \pm 20 \mathrm{~mJ}$ & $380-540 \mathrm{~nm}$ \\
& $150 \mathrm{~mJ} \pm 30 \mathrm{~mJ}$ & $540-760 \mathrm{~nm}$ \\
Repetitionsrate & abhängig vom Pumplaser, $\leq 50 \mathrm{~Hz}$ &
\end{tabular}




\subsubsection{IR-Lasersystem}

Die Erzeugung der im Bereich von $1350 \mathrm{~nm}$ - $5000 \mathrm{~nm}$ durchstimmbaren Laserstrahlung wird mittels eines Nd:YAG gepumpten Laservision OPO/OPA Systems realisiert, das im Detail bereits in der Dissertation von Dr. Richard Forck und in der Masterarbeit von Christoph Pradzynski beschrieben wurde 41, 78. Im Folgenden sollen die einzelnen Elemente kurz dargestellt werden.

\subsubsection{Nd:YAG-Pumplaser Powerlite 8000}

Der Pumplaser für das im IR-Bereich durchstimmbare Lasersystem stammt ebenfalls von der Firma Continuum (Typ Powerlite Precision II 8000). Der Aufbau ist dem in Abschnitt 3.2.1.2 beschriebenen Lasersystem ähnlich und in Abbildung 3.13 dargestellt. Die Abmessungen der Nd:YAG Kristalle im Oszillator und Verstärker sind identisch, jedoch besitzt er nur einen Verstärker. Der Laser wird mit der Fundamentalen $(1064 \mathrm{~nm})$ betrieben und verfügt über einen Seeding-Laser (Continuum Typ SI-2000), der für eine schmalere Linienbreite der im Laservision OPO/OPA erzeugten Strahlung sorgt. Der injection seeder ist ein im Bereich von $1030 \mathrm{~nm}$ - $1080 \mathrm{~nm}$ durchstimmbarer Faserlaser, dessen Licht ungefähr sechs Größenordnungen intensiver ist als das spontan emittierte Licht im Laserkopf des Oszillators. Er wird mit einer Wellenlänge von 1064,149 nm betrieben. In Verbindung mit der Pockels-Zelle wird so zunächst eine hohe Besetzungsinversion im Oszillator erzeugt und schließlich mit Hilfe des Seeders eine longitudinale Mode des Übergangs ${ }^{4} \mathrm{~F}_{3 / 2}$ zu ${ }^{4} \mathrm{I}_{11 / 2}$ stimuliert emittiert. Eine weitere Verstärkung des Laserlichts erfolgt mit einem zweiten, durch Blitzlampen gepumpten Laserkopf. Für das optische Pumpen des Laservision OPO/OPA-Systems muss die Energie des horizontal polarisierten Lichts auf $550 \mathrm{~mJ}$ pro Puls reduziert werden.

\subsubsection{LaserVision OPO/OPA}

Über zwei Spiegel gelangen die $1064 \mathrm{~nm}$ des Nd:YAG-Lasers in den LaserVision OPO/OPA, dessen Aufbauskizze in Abbildung 3.14 dargestellt ist. Im Gegensatz zum Panther EX OPO verfügt dieser zusätzlich über einen optisch parametrischen Verstärker (engl. amplifier, OPA), in dem eine Frequenzmischung des im OPO erzeugten idlers mit der Fundamentalen des YAGs durchgeführt wird. 


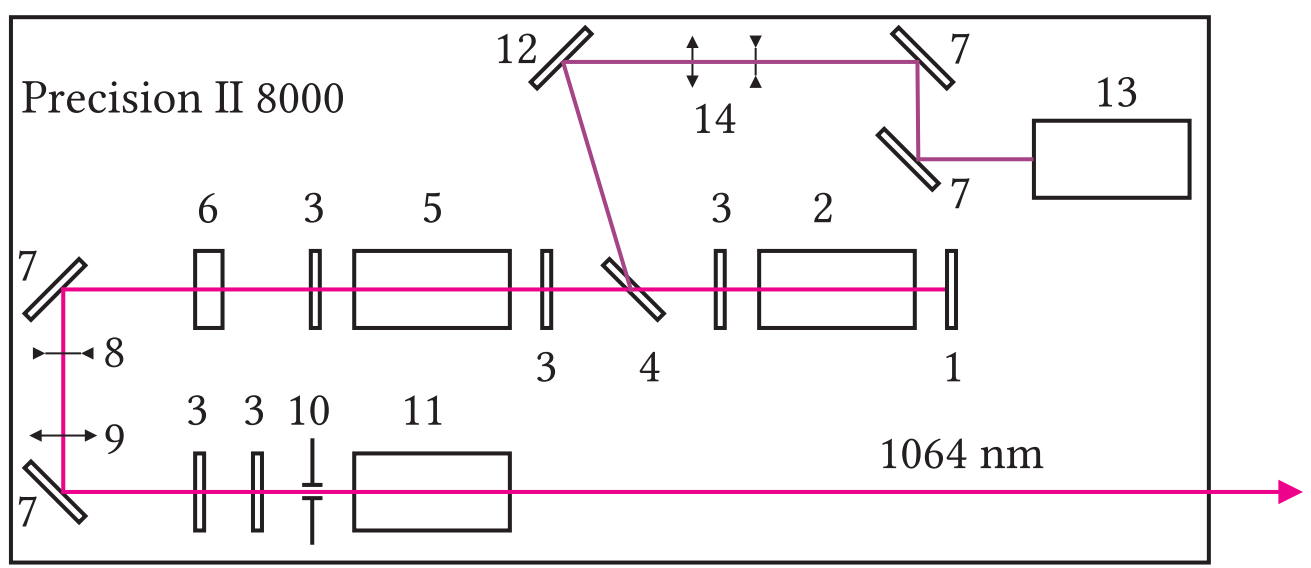

Abbildung 3.13.: Aufbauskizze des Continuum Precision II 8000 Lasers entnommen aus 41, verändert nach 137.
1. Endspiegel
8. Divergente Linse
2. Pockels-Zelle
9. Konvergente Linse
3. $\lambda / 4$-Platte
10. Lochblende
4. Dielektrischer Polarisator
11. Laserkopf mit Blitzlampen
5. Laserkopf mit Blitzlampen
12. $32^{\circ}$-Umlenkspiegel
6. Auskoppelspiegel
13. Seeder
7. $45^{\circ}$-Umlenkspiegel
14. Seederteleskop

Durch eine Iris und ein Teleskop gelangt das Laserlicht auf einen Strahlteiler der 1/3 der Fundamentalen in Richtung des OPOs reflektiert, während der andere Teil den Spiegel in Richtung OPA passiert. Da der OPO als Pumpwellenlänge $532 \mathrm{~nm}$ benötigt, wird die Fundamentale des Nd:YAGs über einen KaliumtitanylphosphatKristall $\left(\mathrm{KTiOPO}_{4}[\mathrm{KTP}]\right)$ frequenzverdoppelt. Die Intensität des resultierenden Lichts, welches vertikal polarisiert ist, kann mittels einer verstellbaren $\lambda / 2$-Platte vor dem Verdopplungskristall reguliert werden. Über drei dichroitische Spiegel gelangt das frequenzverdoppelte Licht in den OPO während der Rest der $1064 \mathrm{~nm}$ den ersten Spiegel passiert und auf einen Strahlfänger trifft. Im OPO wird die gewünschte Wellenlänge, welche sich hier auf den idler bezieht, durch die Verkippung der beiden KTP-Kristalle in einem Dreiwellenprozess von Typ II gebildet. 


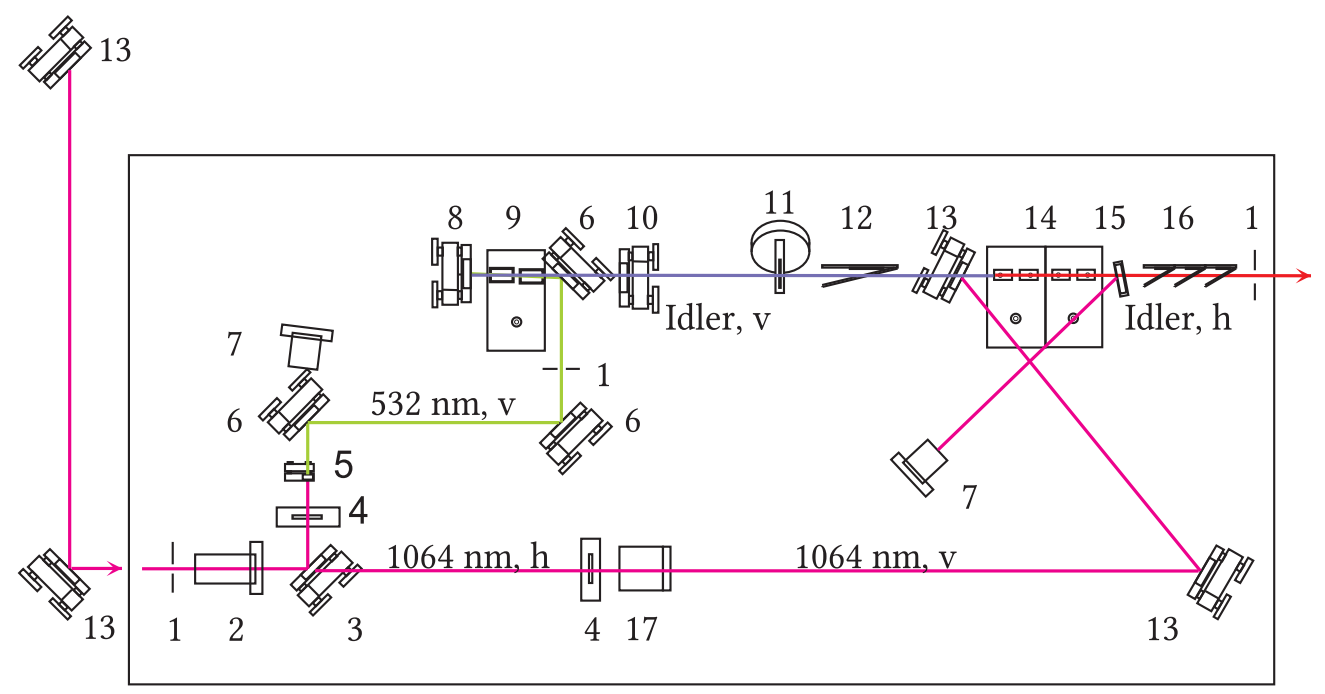

Abbildung 3.14.: Aufbauskizze des LaserVision OPO/OPA-Systems [138], h: horizontal polarisiertes Licht, v: vertikal polarisiertes Licht.

1. Iris

2. Teleskop

3. Strahlteiler $(1 / 3$ zu $2 / 3)$

4. einstellbare $\lambda / 2$ Platte

5. KTP Verdopplungskristall

6. Umlenkspiegel $532 \mathrm{~nm}$

7. Strahlfänger

8. Hinterer Spiegel

9. KTP Kristalle des OPOs
10. Auskoppelspiegel

11. $\lambda / 2$ Platte

12. SI Filter

13. Umlenkspiegel $1064 \mathrm{~nm}$

14. KTA Kristalle des OPAs

15. Dichroitischer Spiegel

16. Brewster-Fenster

17. Dünnschicht-Polarisator

Pumpwellenlänge und idler sind dabei vertikal polarisiert, das signal weist eine horizontale Polarisation auf. Nach dem Verlassen des Oszillators wird die Polarisation über eine $\lambda / 2$-Platte um $90^{\circ}$ gedreht.

Im OPA kommen Kalium-Titanyl-Arsenat-Kristalle $\left(\mathrm{KTiOAsO}_{4}[\mathrm{KTA}]\right)$ zum Einsatz, da sie eine wesentlich höhere Zerstörungsschwelle im IR-Bereich aufweisen. Die Erzeugung der gewünschten Strahlung geschieht mittels Differenzfrequenzmischung aus dem idler des OPOs und dem restlichen Anteil der $1064 \mathrm{~nm}$ aus dem 
Nd:YAG. Dessen Polarisation wird mit Hilfe einer vorgeschalteten $\lambda / 2$-Platte und einem Dünnschicht-Polarisator geändert und zusätzlich wirde das Licht zur Synchronisation mit dem OPO zeitlich verzögert. Die Polarisationsänderung der Ausgangswellenlängen ist nötig, da dadurch die Verkippung der OPA Kristalle in der Vertikalen stattfinden kann. Ein Spiegel hinter dem OPA reflektiert die $1064 \mathrm{~nm}$ und das Brewster-Fenster filtert die verbliebene Strahlung aufgrund ihrer Polarisation. Die Leistungsdaten des Laservision OPO/OPA Systems, entnommen aus dem Betriebshandbuch [138], sind in Tabelle 3.9 abgebildet. Die Angaben sind konservativ, in einigen Wellenlängenbereichen sind bis zu $20 \mathrm{~mJ}$ pro Puls zu erreichen.

Die Steuerung des Lasers erfolgt mit Hilfe eines auf dem Betriebssystem Win 98

Tabelle 3.9.: Kenndaten des LaserVision OPO/OPA-Lasersystems. Entnommen aus Referenz 138 .

\begin{tabular}{c|c|c}
\hline Spezifikation & Wert & Kommentar \\
\hline Bereich & $710-885 \mathrm{~nm}$ und $1.35-5 \mu \mathrm{m}$ & \\
Pumpstrahl & $550 \mathrm{~mJ}$ bei $1064 \mathrm{~nm}$ & horizontal polarisiert \\
Pulsenergie & $12 \mathrm{~mJ}$ & $1.5-3.5 \mu \mathrm{m}$ \\
& $4 \mathrm{~mJ}$ & $4.0 \mu \mathrm{m}$ \\
& $0.5 \mathrm{~mJ}$ & $4.9 \mu \mathrm{m}$ \\
Repetitionsrate & maximal $10 \mathrm{~Hz}$ &
\end{tabular}

basierenden Computerprogramms. Zu beachten ist, dass dem Programm die Wellenlänge des OPOs vorgegeben wird. Es wurde bereits in der Arbeit von Dr. Richard Forck festgestellt, dass eingestellte und tatsächliche Wellenlänge im für das Experiment relevanten Bereich einen linearen Versatz von $12-13 \mathrm{~cm}^{-1}$ aufweisen [41]. Dieser Wert wurde durch Absorptionsmessungen im Vergleich mit FTIRSpektren von Polystyrol im Bereich von 3000 bis $3100 \mathrm{~cm}^{-1}$ und Polyethylen in Bereich von 3590 bis $3670 \mathrm{~cm}^{-1}$ ermittelt 41 und durch Nachmessungen bestätigt.

\subsection{Erzeugung von Messdaten}

Nachdem die einzelnen Bauteile des Experiments erläutert wurden, soll in diesem Abschnitt die Erzeugung der Messdaten näher beschrieben werden. Die Veränderung zum vorhergehenden Experiment [41] werden detaillierter beschrieben. Zuerst 
wird die Synchronisation des veränderten Messablaufs erläutert und schließlich wird die Umwandlung der Massenspektren in ein IR-Spektrum erklärt.

\subsubsection{Synchronisation des Massenspektrometers}

Die Steuerung des Experiments soll anhand der Abbildung 3.15 erläutert werden. Die Generierung und Dotierung der Cluster findet in einer kontinuierlichen Expansion statt. Detektiert werden im Massenspektrometer jedoch nur Ionen, die gepulst erzeugt werden. Die Verarbeitung der Massensignals findet mit Hilfe der Vielkanalzählerkarte P7887 der Firma FAST ComTec GmbH statt [139]. Diese ist in einem PCI-Steckplatz des Messcomputers verbaut. Die Steuerung erfolgt mit Hilfe des auf Win 7 basierenden Programms MCDWIN. Nähere Erläuterungen finden sich in der Arbeit von Dr. Forck [41].

Das Start-Signal für jede Messung wird über einen UV/Vis-Laserpuls, der von einer Photodiode am Einkoppelfenster erfasst wird, geliefert. Die Karte registriert über den Chevron MCP-Detektor die auftreffenden, über ihre Flugzeit separierten Ionen. Die maximale Auflösung eines Kanals beträgt dabei 250 ps. Um die entstehende Datenmenge zu reduzieren werden 32 Kanäle zusammengefasst, wodurch eine Auflösung von $8 \mathrm{~ns}$ pro Kanal resultiert. Ein Laserpuls erzeugt ein Massenspektrum. Um die Statistik zu verbessern, werden 1000 Massenspektren zusammengefasst und als asc-Datei gespeichert. Eine weitere Datei mit der Endung Pr887 enthält die vorher editierten Bedingungen (eingestellte Temperaturen von Ofen, Düse, PickUp-Zelle oben/unten, verwendetes Seedinggas mit Druck, Laserwellenlängen und Photonendichten etc.) der aufgenommen Messung.

\subsubsection{Synchronisation der Lasersysteme}

Die meisten hier dargestellten experimentellen Ergebnisse wurden mit Hilfe des alten Excimer-/Farbstofflasersystems erzeugt. Die Pulslänge von 30 ns und der auftretende Jitter sorgten dafür, dass die maximale Verstärkung im IR-CIPI Experiment bei einer zeitlichen Verzögerung der Laserpulse von ungefähr 80 ns auftrat. Durch den Austausch des UV/Vis-Lasersystems ist der Jitter-Effekt beseitigt, sodass die maximale Verstärkung von den Pulslängen und dem Energietransfer in den Clustern abhängig sein sollte. 


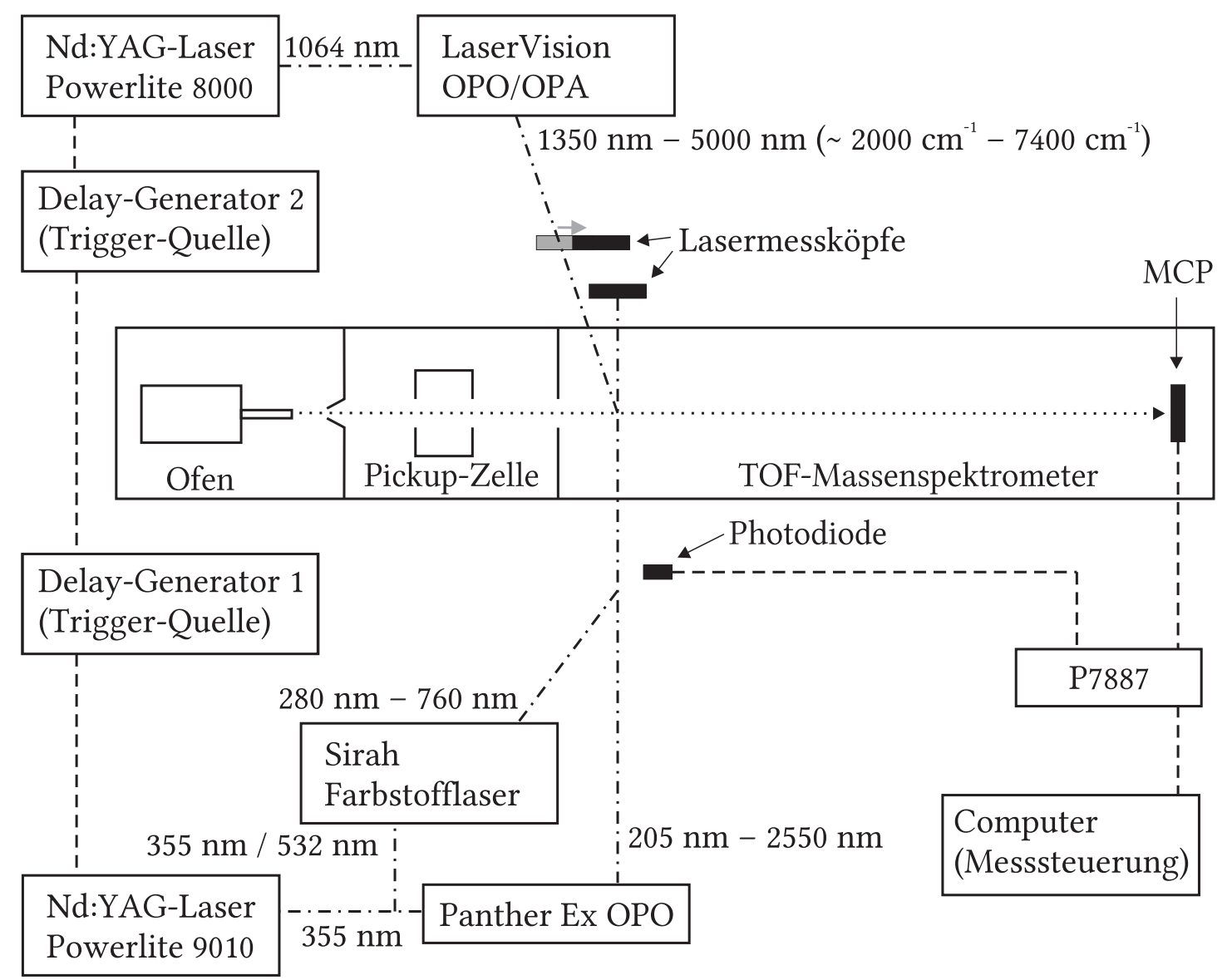

Abbildung 3.15.: Schematische Darstellung zur Steuerung des Experiments, verändert nach 108. Verbindungen: (--) elektrische Leitungen, (-.-) Laserstrahlen, (…) Molekularstrahl.

Um einen möglichst genauen zeitlichen Versatz im Zwei-Farben-Experiment zu gewährleisten, werden beide Pumplaser extern über jeweils einen Stanford DG535 Delay-Generator getriggert (vgl. Abb. 3.15). Der Excimerlaser benötigte lediglich einen $10 \mu \mathrm{s}-100 \mu \mathrm{s}$ langen Puls mit einer aufsteigenden Flanke von 3,3 V-15 V. Hier war der Anschluss an einen der vier Kanäle des DG535 ausreichend. Bei den Nd:YAG-Lasern werden Blitzlampen und Q-Switch separat getriggert. Sie benötigen einen $10 \mu$ s langen Puls mit einer ansteigenden Flanke von $>2 \mathrm{~V}$, die über zwei Kanäle, A und B für die Blitzlampen bzw. C und D für den Q-Switch, aus dem DG535 modelliert werden. Die Tabelle 3.10 stellt die eingestellten Werte für ein Delay von $80 \mathrm{~ns}$ zwischen neuem UV/Vis- und IR-Laser dar. $\mathrm{T}_{0}$ bezeichnet den Startpunkt. Der Delay-Generator des UV/Vis Lasersystems triggert über $T_{0}$ 
den Delay-Generator des IR-Lasersystems. Beim Excimer gepumpten UV/VisLasersystem musste der Triggerpuls ungefähr 270 us vor der Zündung der Blitzlampen erfolgen, was die Zeit des Q-Switch Delays darstellt. Um die Verzögerung der Laserstrahlen zu messen, werden beide mit einer Photodiode (EOT Model ET-2000) erfasst und mit Hilfe eines Oszilloskops vermessen.

Als Modifikation soll ein Schuss-zu-Schuss Modus etabliert werden. Bei diesem wird das UV/Vis-System bei $10 \mathrm{~Hz}$ und das IR-Lasersystem bei $5 \mathrm{~Hz}$ betrieben. Erste Untersuchungen dieses Modus wurden von Sabine Wolff in ihrer Masterarbeit durchgeführt [140]. Weiterhin wurde in dieser Arbeit die Zeitabhängigkeit der IR-induzierten Signalzunahme bei Verwendung des neuen UV/Vis-Lasersystems untersucht. Für die Ionisation bei $398 \mathrm{~nm}$ steigen die Zunahmen bis zu einem Delay von 40 ns. Ab 100 ns findet ein Abfall der Signalzunahme statt, sodass die hier aufgeführten $80 \mathrm{~ns}$ im Maximalbereich der IR-induzierten Signalzunahme liegen [140].

Tabelle 3.10.: Parameter für die externe Triggerung von IR- und UV/VisLasersystem mit zwei Stanford DG535 Delay-Generatoren bei einer zeitlichen Verzögerung der Laserstrahlen an der Apparatur von 80 ns. $\mathrm{T}_{0}$ bezeichnet den Startpunkt.

\begin{tabular}{c|c|c}
\hline Kanal & Wert UV & Wert IR \\
\hline $\mathrm{A}$ & $\mathrm{T}_{0}+20,09 \mu \mathrm{s}$ & $\mathrm{T}_{0}+345 \mathrm{~ns}$ \\
$\mathrm{~B}$ & $\mathrm{~A}+10 \mu \mathrm{s}$ & $\mathrm{A}+10 \mu \mathrm{s}$ \\
$\mathrm{C}$ & $\mathrm{A}+260 \mu \mathrm{s}$ & $\mathrm{A}+270 \mu \mathrm{s}$ \\
$\mathrm{D}$ & $\mathrm{C}+10 \mu \mathrm{s}$ & $\mathrm{C}+10 \mu \mathrm{s}$
\end{tabular}

\subsubsection{Datenverarbeitung}

Die von dem Auswertungsprogramm P7887 erzeugten asc-Dateien, welche die einzelnen aufsummierten Massenspektren enthalten, wurden mit Hilfe einer von Dr. Richard Forck 41 übernommenen und an den Größenbereich angepassten Matlab-Routine, die im Anhang zu finden ist, ausgewertet (Kapitel A.4 auf Seite VII). Das erste Skript diente der Auswertung des aufgelösten Massenbereichs bis $n=80$ und übergibt in einer zweiten Datei die Eichparameter für jedes Massenspektrum. Mit Hilfe dieser Eichparameter können mit einem Tabellenkalkulationsprogramm Kanalbereiche für Clustergrößen mit mehr als 80 Monomerein- 
heiten bestimmt werden. Die einzelnen, natriumdotierten Wassercluster sind ab dieser Größe bei den vorliegenden Bedingungen nicht mehr aufgelöst. Das zweite Skript diente der Integration der Massenbereiche, die über mehrere Clustergrößen aufsummiert werden, um das Signal-zu-Rausch-Verhältnis zu verbessern. Beide Skripte beinhalten Normierungsfaktoren für die UV/Vis-Photonendichte und die Anzahl der aufgenommenen Massenspektren.

Die weitere Bearbeitung erfolgte mit einem Tabellenkalkulationsprogramm. Im Messbetrieb wurden in der Regel ein reines UV/Vis-Spektrum und drei Spektren mit IR-Lasereinkopplung nacheinander aufgenommen. Die Auflösung betrug in der Regel $10 \mathrm{~cm}^{-1}$, lediglich der Bereich der freien OH-Streckschwingung zwischen $3688 \mathrm{~cm}^{-1}$ und $3718 \mathrm{~cm}^{-1}$ wurde in einigen IR-Spektren in $5 \mathrm{~cm}^{-1}$ Schritten vermessen. Eine Übersicht der Messbedingungen unter denen die für diese Arbeit verwendeten Spektren erzeugt wurden, ist im Anhang (Kapitel A.3 in Tabelle A.2 zu finden. Für jede einzelne Wellenlänge wird das IR-Signal aus den Quotienten der Massenspektren mit IR-Lasereinkopplung und dem Mittelwert aus zwei benachbarten UV/VIS-Massenspektren berechnet. Die IR-Wellenlänge des Laservision OPO/OPA sind 12 bis $13 \mathrm{~cm}^{-1}$ zu größeren Wellenzahlen verschoben, daher wird von den eingestellten IR-Wellenlängen $12 \mathrm{~cm}^{-1}$ abgezogen.

Erfolgt keine weitere Bearbeitung der Messdaten, werden die IR-induzierten Zunahmen als Rohdaten bezeichnet. Zur besseren Darstellung und Vergleichbarkeit der Daten wurden zwei Mittelungsmethoden eingesetzt. Zum einen wurde eine Dreiecksglättung nach folgender Formel durchgeführt:

$$
I_{\tilde{\nu}, i}=\frac{I_{\tilde{\nu}, i-1}+1 / 2 \cdot I_{\tilde{\nu}-1, i-1}+1 / 2 \cdot I_{\tilde{\nu}+1, i-1}}{2} \text { mit } i>0
$$

Dabei stellt $I_{\tilde{\nu}, 0}$ den oben genannten Ausgangsquotienten (Rohdaten) dar, der mit den beiden benachbarten Werten $I_{\tilde{\nu} \pm 1,0}$ den einfach geglätteten Quotienten $I_{\tilde{\nu}, 1}$ bildet. Die Glättung wurde je nach Ausgangssignal bis zu 15 mal durchgeführt. Bei dieser Art der Mittlung gehen viele spezifische Absorptionsmuster verloren, daher eignet sie sich dafür, Trends bei geringem Signal hervorzuheben, wie z.B das in Kapitel 5 diskutierte Einsetzen der Kristallisation.

Eine weitere Möglichkeit ist die Zusammenführung mehrere Rohdatenscans bei gleichen Expansionsbedingungen. Hierfür werden zunächst die Zunahmen auf das maximale Signal $[79 \mid$ oder das Gesamtintegral normiert [80 und schließlich der 
Mittelwert bei jeder IR-Wellenlänge gebildet. Anschließend können wieder eine oder mehrere Dreiecksglättungen durchgeführt werden. Die Anzahl der verwendeten Scans und Glättungen wird für das gezeigte Spektrum jeweils im Text und in der Abbildung angegeben. 


\section{Vergleich mit weiteren experimentellen Methoden}

Dieses Kapitel soll die im Rahmen dieser Arbeit erhaltenen IR-Spektren mit jenen anderer experimenteller Methoden in unterschiedlichen Größenbereichen vergleichen und die Vielseitigkeit der hier eingesetzten experimentellen Methode verdeutlichen. Dabei wird insbesondere folgenden Fragen nachgegangen: Wie viel Einfluss hat das Chromophor Natrium auf die Wassercluster mit 19 und mehr Monomereinheiten? Ab welcher Clustergröße ähneln sich die IR-Spektren neutraler und ionischer Cluster? Welche Vorteile, aber auch Nachteile bieten massenselektive Methoden gegenüber Techniken, die auf der direkten Messung von Lichtintensitäten beruhen?

Zuerst erfolgt die Gegenüberstellung von zwei größenselektiven Methoden. Eine ist die Dotierung mit Phenol, was die Untersuchung von Wasserclustern im Größenbereich $n=20-50$ [37 ermöglicht. Die andere ist die Erzeugung ionischer Cluster der Clustergröße $n=250$ [26. Zum Abschluss erfolgt der Vergleich der Spektren von massenselektierten, kristallinen Wasserclustern der Größe $n=575 \pm 25$ mit ebenfalls kristallinen, $12 \mathrm{~nm}$ großen Partikeln, was einem Cluster mit ungefähr 27000 Molekülen entspricht [43,44], die mit Hilfe direkter Absorption gemessen wurden.

\subsection{Phenoldotierte Wassercluster}

Eine größenselektive Methode zur Untersuchung neutraler, wasserstoffbrückengebundener Clustern basiert auf ihrer Dotierung mit Phenol. Dieses verfügt über eine Hydroxylgruppe, die als einfacher Donor und doppelter Akzeptor für Wasserstoffbrückenbindungen fungieren kann. Aufgrund der sterischen Hinderung durch den aromatischen Sechsring und der geringeren Koordination als Wasser liegt 
das Phenol auf der Oberfläche des entstehenden Clusters vor. Wie in zahlreichen Studien gezeigt, sind die so entstehenden Wasserstoffbrückentopologien der Phenol- $\left(\mathrm{H}_{2} \mathrm{O}\right)_{n}$-Cluster schon bei kleinen Clustergrößen $(n=1-8)$ denen von reinen $\left(\mathrm{H}_{2} \mathrm{O}\right)_{n+1}$-Clustern ähnlich $35,141-144$.

Der Gruppe von Fujii gelang es als erste überhaupt IR-Spektren von Phenol$\left(\mathrm{H}_{2} \mathrm{O}\right)_{n}$-Clustern im Größenbereich von 20 bis 50 Wassermolekülen zu messen [36, 37] und damit Aussagen über neutrale Wassercluster dieses Größenbereichs aufgrund experimenteller Belege treffen zu können. Dieses Experiment soll im Folgenden kurz vorgestellt werden.

\subsubsection{Experimentelle Methode}

Die phenoldotierten Cluster werden mittels eines temperierbaren Sättigers in einer gepulsten Überschallexpansion erzeugt 145. Die Ionisation erfolgt mit Hilfe der resonanten Zweiphotonenionisation $(1+1)$ R2PI bei einer Wellenzahl von $36254 \mathrm{~cm}^{-1}$ (ungefähr $276 \mathrm{~nm}$ ) in einem TOF-Massenspektrometer [36, 37. Aufgrund der Überschussenergie erfolgt ein Abdampfen von Wassermolekülen nach der Ionisation, was zu einer Unsicherheit zwischen der Ausgangsspezies Phenol$\left(\mathrm{H}_{2} \mathrm{O}\right)_{n+\Delta n}$ und der detektierten Spezies Phenol- $\left(\mathrm{H}_{2} \mathrm{O}\right)_{n}^{+}$von $0 \leq \Delta n \leq 6$ führt [36]. Die Cluster werden 50 ns vor der Ionisation mit Hilfe eines IR-Pulses angeregt. Dieser führt zu einer Depopulierung des Grundzustandes und verringert somit die Effizienz der Photoionisation. Das IR-Spektrum des Phenol- $\left(\mathrm{H}_{2} \mathrm{O}\right)_{n+\Delta n}$-Clusters kann somit über die Abnahme des Ionensignals des dazugehörigen, einfach geladenen Kations im Massenspektrum erhalten werden. Das verwendete IR-Lasersystem weist eine Lücke um $3500 \mathrm{~cm}^{-1}$ auf, ausgelöst durch Wasserverunreinigungen im $\mathrm{LiNbO}_{3}$-Kristall. Um den Einfluss der Fragmentierung von größeren Clustern zu mindern, werden die Expansionen so angepasst, dass die zu untersuchende Clustergröße am äußeren Rand des Massenverteilung liegt. 


\subsubsection{Vergleich der IR-Spektren von phenol- und natriumdotierten Clustern im Größenbereich $n=20-50$}

Der Vergleich der IR-Spektren der phenol- und natriumdotierten Cluster der Größen $n=19,29,39,49$ ist in Abbildung 4.2 dargestellt. Abbildung 4.3 vergleicht die IR-Spektren der phenoldotierten Cluster der erwähnten Größen mit natriumdotierten Clustern, die ein zusätzliches Wassermolekül enthalten. Die IR-Spektren im natriumdotierten Fall wurden mit einer Aufösung von $5 \mathrm{~cm}^{-1}$ im gesamten $\mathrm{OH}-$ Streckschwingungsbereich an mehreren Tagen (02.06.2010, 04.06.2010, 07.06.2010 und 08.06.2010) aufgenommen. Die höhere Auflösung führt zu einer besseren Vergleichbarkeit. Die genauen Parameter und Wellenlängenbereiche können der Tabelle A.2 in Kapitel A.3 entnommen werden.

Ein Massenspektrum dieser Messreihe bei einer Ionisationswellenlänge vom $400 \mathrm{~nm}$ ist in Abb. 4.1 abgebildet. Die Expansions- und Ionisationsparameter führen zu einer schmalen Clusterverteilung beginnend bei $n=4$ mit einem Maximum bei $n=28$. Vorherige Arbeiten haben gezeigt, dass bei der vorliegenden Verteilung eine Auswertung der IR-Spektren bis zu einer Clustergröße von $n=50$ möglich ist [78].

Die IR-Spektren der natriumdotierten Cluster in Abbildung 4.2 und 4.3 sind zweifach geglättet, bei den Punkten wurde eine einfache Glättung durchgeführt (vgl. Kap. 3.3.3. Die genaueren Auswertungsmodalitäten können [78 entnommen werden. Betrachtet man die IR-Spektren natriumdotierter Cluster untereinander (jeweils rot gekennzeichnete Spektren in Abb. 4.2 und 4.3), so weisen alle Clustergrößen um $3150 \mathrm{~cm}^{-1}$ und $3550 \mathrm{~cm}^{-1}$ einen Signaleinbruch im Spektrum auf. Dieser ist jeweils nur durch einen Messwert begründet, der allerdings nicht systematisch bei einer Wellenzahl liegt. Die Spektren zeigen ein breites Absorptionsmuster zwischen $3000 \mathrm{~cm}^{-1}$ und $3600 \mathrm{~cm}^{-1}$, dessen Verlauf sich jedoch für verschiedene Clustergrößen unterscheidet. Das Absorptionsmaximum der freien OH-Streckschwingung liegt bei den vorliegenden Clustergrößen, die in in einer Heliumexpansion erzeugt wurden, bei $3708 \mathrm{~cm}^{-1}$.

Beim Vergleich der IR-Spektren zwischen Phenol- $\left(\mathrm{H}_{2} \mathrm{O}\right)_{n}$ und $\mathrm{Na}\left(\mathrm{H}_{2} \mathrm{O}\right)_{n}$ wird ersichtlich, dass die phenoldotierten Cluster mit einer höheren Auflösung gemessen wurden, jedoch zeigen sie den gleichen Absorptionsverlauf mit einem Anstieg ab $3000 \mathrm{~cm}^{-1}$ und einem Abfall bei $3550 \mathrm{~cm}^{-1}$. Das Signal-zu-Rausch-Verhältnis un- 


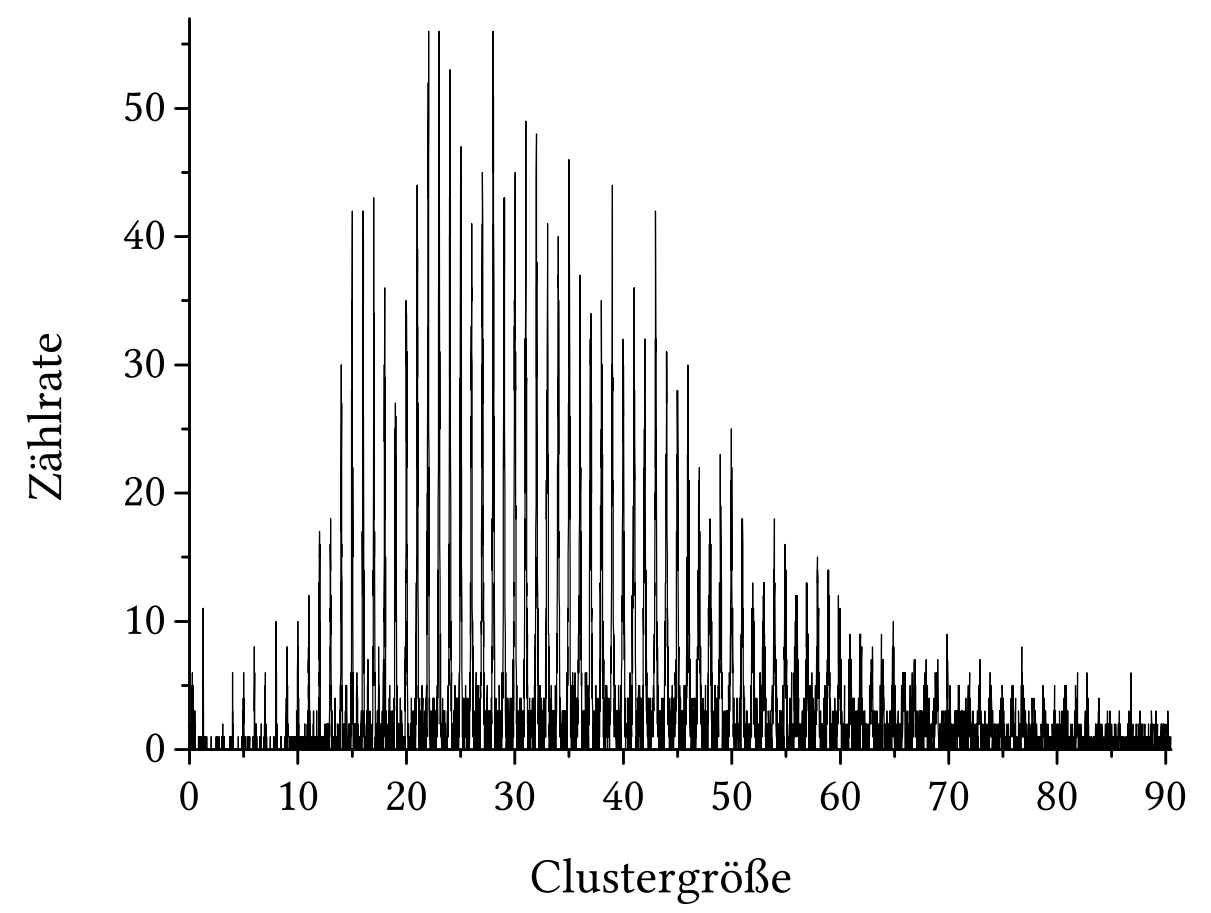

Abbildung 4.1.: Massenspektrum vom 04.06.2010 der natriumdotierten Cluster bei einer Ionisationswellenlänge von $400 \mathrm{~nm}$. Weitere Parameter der Expansion sind in Tab. A.2 im Anhang zu finden.

terscheidet sich nur gering. Während die Clustergrößen $n=19,29$ ihr Absorptionsmaximum oberhalb von $3400 \mathrm{~cm}^{-1}$ haben, weist die Absorption von $n=39,49$ ein Plateau zwischen $3200 \mathrm{~cm}^{-1}$ und $3400 \mathrm{~cm}^{-1}$ auf. Dies deutet auf eine verstärkte Zunahme von DDAA-Oszillatoren gegenüber DDA- bzw. DAA-Oszillatoren hin [37] (vgl. Tabelle 2.1 auf Seite 12). Die Absorptionsmuster der freien $\mathrm{OH}-$ Streckschwingung sind in allen Größenbereichen ähnlich und zeigen ein lokales Absorptionsmaximum bei ca. $3708 \mathrm{~cm}^{-1}$. Die Linienbreite dieses Peaks im Fall der phenoldotierten Cluster scheint geringer und es findet auch eine systematische Intensitätsabnahme mit der Clustergröße statt. Ein Grund für diesen Unterschied könnte an der durchgeführten Glättung bei den Spektren der natriumdotierten Cluster liegen. Hervorzuheben sind die übereinstimmenden Bandenschemata für $n=19$ um $3050 \mathrm{~cm}^{-1}$ und für $n=39$ zwischen $3080 \mathrm{~cm}^{-1}$ und $3200 \mathrm{~cm}^{-1}$ (in der Abb. 4.2 in grün hervorgehoben). Diese liegen im Bereich der auf der Oberflä- 

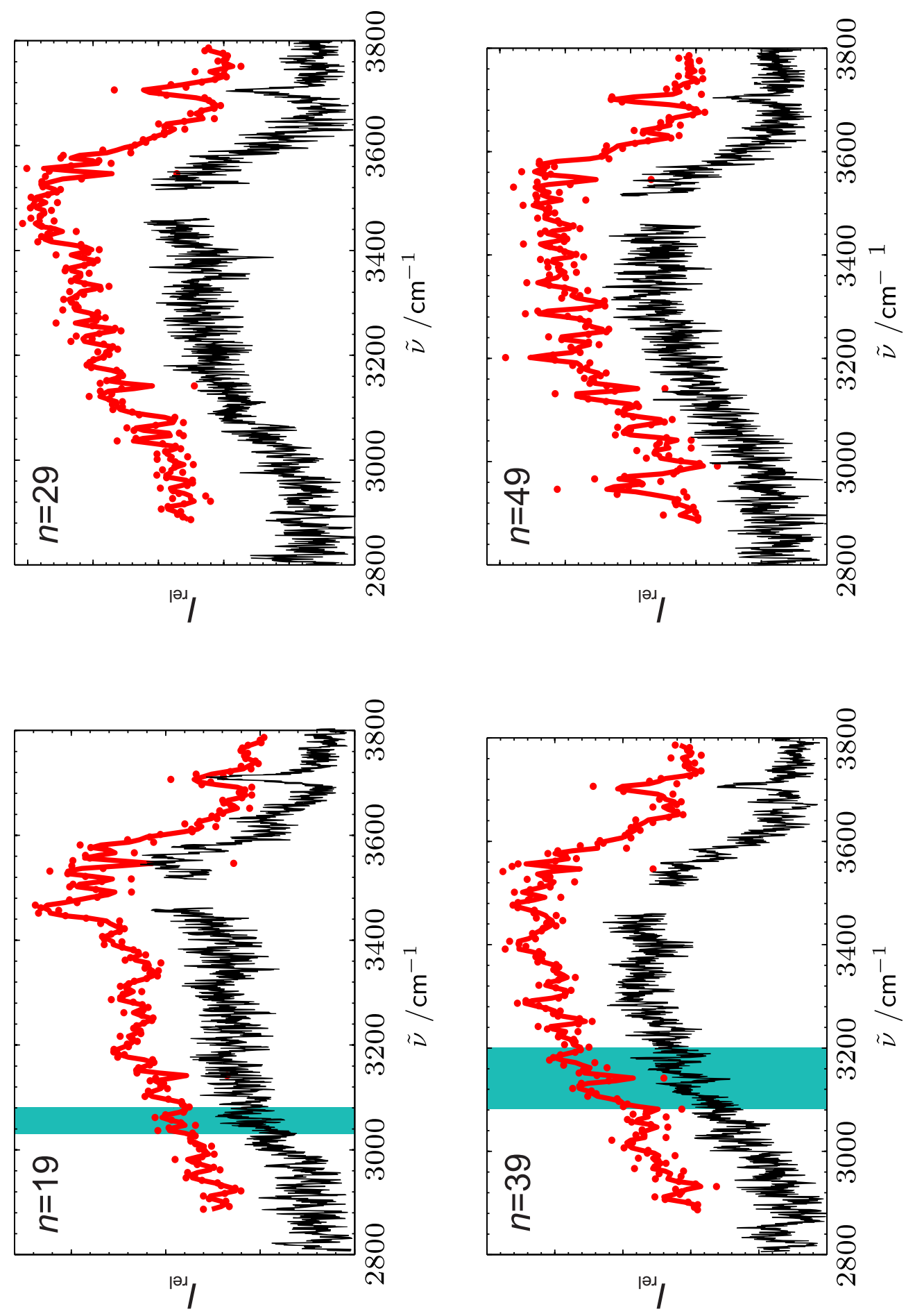

Abbildung 4.2.: Vergleich der IR-Spektren von Phenol- $\left(\mathrm{H}_{2} \mathrm{O}\right)_{n}|37|$ und $\mathrm{Na}\left(\mathrm{H}_{2} \mathrm{O}\right)_{n}$ der Größen $n=19,29,39,49$. Die Daten von $\mathrm{Na}\left(\mathrm{H}_{2} \mathrm{O}\right)_{n}$ stammen aus den Messtagen vom 02.06.2010, 04.06.2010, 07.06.2010 und 08.06.2010 (vgl. Tabelle A.2 auf VI). 
che der Cluster lokalisierten DAA-Oszillatoren. Das schlechte Signal-zu-RauschVerhältnis der Spektren für $n=49$ erschwert einen Vergleich. Aufgrund der verwendeten Ionisationswellenlänge von $400 \mathrm{~nm}$ könnte ein signifikanter Absorptionsanteil von Isomeren mit solvatisiertem Natrium die Unterschiede im spektralen Verlauf für die Clustergrößen $n=19,29$ und $39 \mathrm{um} 3400 \mathrm{~cm}^{-1}$ erklären. Diese Absorption könnte durch die Interaktion der Oszillatoren mit dem 3s-Elektron des Natriums verursacht werden $[39$.

Im Gegensatz zu Natrium bildet Phenol bis zu drei Wasserstoffbrückenbindungen aus und kann somit als zusätzlicher DAA-Oszillator fungieren. Um diesen Einfluss auf die $\mathrm{OH}-$ Brückentopologie näher zu beleuchten, erfolgt eine Gegenüberstellung der IR-Spektren der phenoldotierten Cluster aus dem vorhergehenden Abschnitt mit natriumdotierten Clustern mit einem zusätzlichen Wassermolekül in Abbildung 4.3. Die im vorherigen Vergleich ,auffälligen“ Bereiche „großer“ Übereinstimmung bei $n=19$ und $n=29$ sind in der Abbildung blau hervorgehoben. Die Spektren zwischen den beiden Chromophoren weisen zum Teil ähnliche Absorptionsmuster, z.B. für $n=19 / 20$ bei $3320 \mathrm{~cm}^{-1}$ auf. Die Übereinstimmung zwischen den Clustern mit gleicher Wassermolekülanzahl ist jedoch „besser“. Die Differenzen der Absorption bei $3400 \mathrm{~cm}^{-1}$ und der freien OH-Streckschwingung werden durch ein zusätzliches Wassermolekül nicht kompensiert. Phenol- $\left(\mathrm{H}_{2} \mathrm{O}\right)_{29}$ zeigt in beiden Fällen ähnliche Abweichungen, jedoch ist hier die Ähnlichkeit der Absorption der freien OH-Schwingung für 30 Wassermoleküle besser. Für $n=39$ ist eine Übereinstimmung in beiden natriumdotierten Fällen zu beobachten. Das IRSpektrum von $\mathrm{Na}\left(\mathrm{H}_{2} \mathrm{O}\right)_{40}$ zeigt, ähnlich wie $\mathrm{Na}\left(\mathrm{H}_{2} \mathrm{O}\right)_{39}$, das Absorptionsmuster zwischen $3100 \mathrm{~cm}^{-1}$ und $3300 \mathrm{~cm}^{-1}$, welches dem DAA-Motiv zugeordnet werden kann. In diesem Größenbereich scheinen sich die IR-Spektren der benachbarten Clustergrößen kaum zu unterscheiden. Das Signal-zu-Rausch-Verhältnis in dem natriumdotiertem Spektrum erschwert die Zuordnung für Phenol- $\left(\mathrm{H}_{2} \mathrm{O}\right)_{49}$, jedoch gibt es bei $\mathrm{Na}\left(\mathrm{H}_{2} \mathrm{O}\right)_{50}$ mehr Unterschiede im Absorptionsmuster der freien $\mathrm{OH}$ Streckschwingung.

Der Vergleich zwischen natrium- und phenoldotierten Clustern zeigt, dass bereits bei Clustergröße $n=19$ beide Methoden ähnliche IR-Spektren liefern. Die wesentliche Änderung der größenselektiven Spektren mit steigender Molekülanzahl aus der Pionierarbeit von Hamashima et al. [37] ist ein Wachstum der relativen Absorption zwischen $3200-3400 \mathrm{~cm}^{-1}$, was auf eine Zunahme des Anteils der vierfachkoordi- 

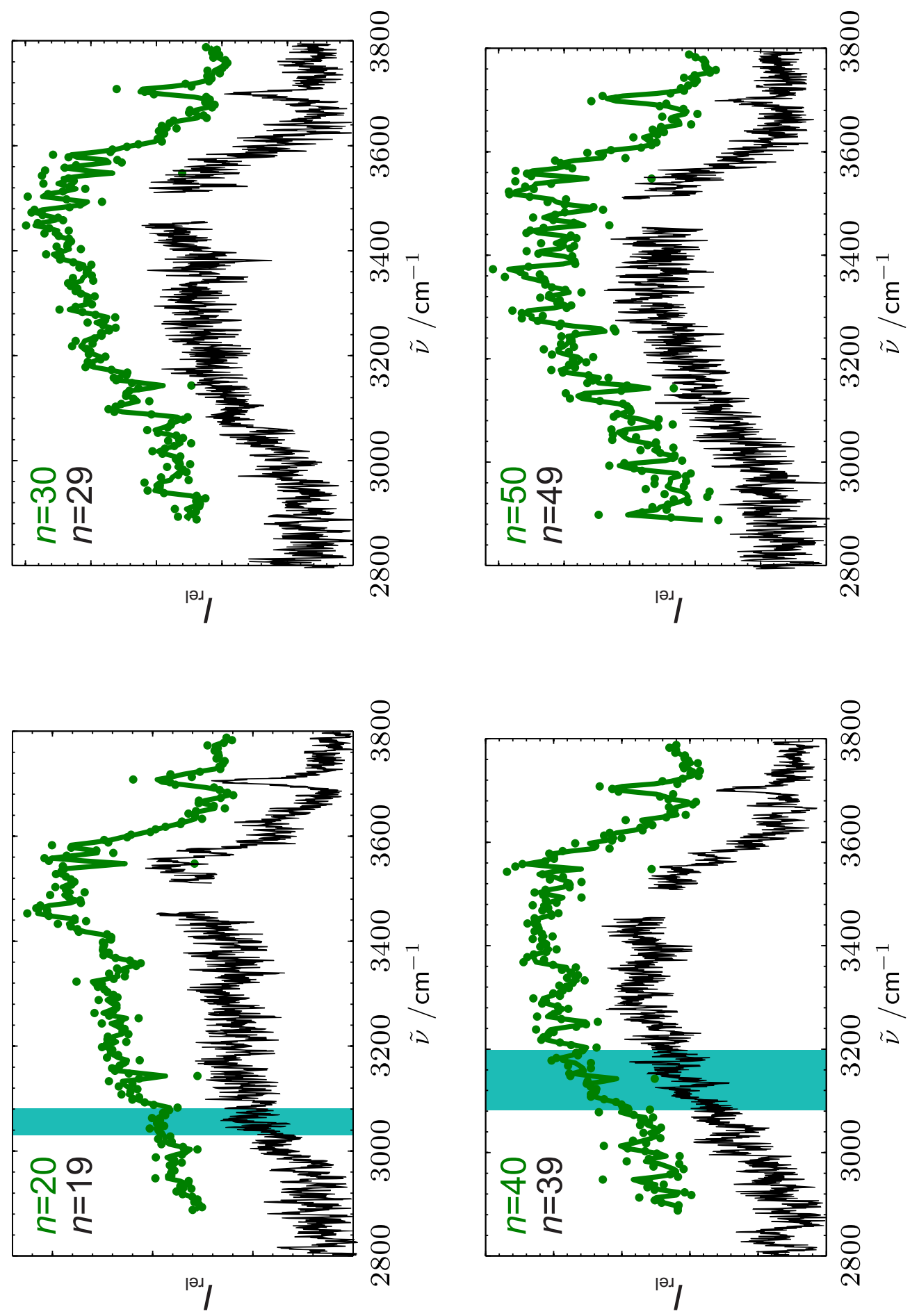

Abbildung 4.3.: Vergleich der IR-Spektren von Phenol- $\left(\mathrm{H}_{2} \mathrm{O}\right)_{n}|37|$ und $\mathrm{Na}\left(\mathrm{H}_{2} \mathrm{O}\right)_{n}$ der Größen $n=19,29$, 39, 49 für phenoldotierte und $n=20$, 30, 40, 50 für natriumdotierte Cluster. Die Daten von $\mathrm{Na}\left(\mathrm{H}_{2} \mathrm{O}\right)_{n}$ stammen aus den Messtagen vom 02.06.2010, 04.06.2010, 07.06.2010 und 08.06.2010 (vgl. Tabelle A.2 auf VI). 
nierter Moleküle deutet. Dieser Befund kann mit der hier verwendeten, unabhängigen, experimentellen Methode bestätigt werden. Die größte Störung durch das Natriumatom würde man für die zwei- und dreifach koordinierten Wassermoleküle auf der Oberfläche des Clusters erwarten, doch beide Methoden weisen hier eine sehr gute Übereinstimmung auf, da Absorptionsmuster wie bei $n=39$ ähnlich sind. Die geringen Unterschiede im Bereich um $3400 \mathrm{~cm}^{-1}$ könnten auf die Wechselwirkungen von OH-Oszillatoren der Wassercluster mit dem 3s-Elektron des Natriums zurückzuführen sein. Aufgrund der Ionisationswellenlänge von $400 \mathrm{~nm}(3,1$ eV) könnte ein Teil der Absorption auf Isomerklasse I zurückzuführen sein 39,78. Diese weist wegen der Separierung von Natrium und 3s-Elektron eine höhere Partialladung und damit eine stärkere $\mathrm{Na}-\mathrm{O}$-Bindung auf, die direkten Einfluss auf den OH-Oszillator und dessen Absorptionswellenlänge hätte. Der Vergleich der IR-Spektren zwischen Phenol- $\left(\mathrm{H}_{2} \mathrm{O}\right)_{n}$ und $\mathrm{Na}\left(\mathrm{H}_{2} \mathrm{O}\right)_{n+1}$ zeigt, dass bei $n=39$ ein zusätzliches Wassermolekül nur geringen Einfluss auf das Absorptionsverhalten und damit die Struktur der Cluster hat. Ein weiterer Grund für die sehr ähnlichen Spektren dieser Clustergröße könnte sein, dass die Unsicherheit der Clustergröße $(\Delta n \leq 6)$, verursacht durch die Fragmentierung der Phenoldotierten Cluster in der Detektion, bei dieser Clustergröße am geringsten ist. Insgesamt weisen die Spektren mit gleicher Wassermolekülanzahl mehr Übereinstimmungen auf. Die erhaltenen Ergebnisse deuten darauf hin, dass die Erzeugung der IR-Wirkungsspektren mittels IR-CIPI im Größenbereich ab $n=19$ zur Strukturaufklärung von reinen Wasserclustern verwendet werden kann, da das Chromophor Natrium nur geringe Auswirkungen auf das $\mathrm{OH}-$ Brückennetzwerk im Cluster hat.

Da ab $n=49$ keine größenselektiven Spektren neutraler Wasseraggregate vorliegen, erfolgt im nächsten Abschnitt ein Vergleich zwischen ionischen und natriumdotierten Clustern.

\subsection{Ionische Wassercluster}

Das erste IR-Absorptionsspektrum von ionischen Wasserclustern in der Gasphase wurde von Schwarz für hydratisierte Hydroniumionen $\mathrm{H}^{+}\left(\mathrm{H}_{2} \mathrm{O}\right)_{n}$ im Jahr 1977 veröffentlicht [146]. Die ionischen Cluster wurden mit Hilfe einer gepulsten Radiolyse von Wasserdampf erzeugt und ihre IR-Absorption direkt gemessen. Eine 
effektive Größenselektion war nicht vorhanden. Die meisten IR-Studien von ionischen Clustern nutzen heutzutage die IR(M)PD Spektroskopie [145. Die Gruppe von Fujii ist bei der Detektion hydratisierter Hydroniumionen bis in den Größenbereich um $n=221$ vorgedrungen [25, 27]. Auf diese Studie wird im Hinblick auf das Einsetzen der Kristallisation in Kapitel 5.3 eingegangen. Johnson und Mitarbeiter haben, mit dem Fokus auf der Interaktion mit dem solvatisierten Elektron, anionische Cluster mit bis zu 50 Wassermolekülen im Bereich von $60-1820 \mathrm{~cm}^{-1}$ mittels IRMPD untersucht 29]. Im Bereich der OH-Streckschwingung sind Studien der Gruppe bis $n=21$ publiziert $30,31,147$. In einer erst kürzlich veröffentlichten Studie untersuchte die Gruppe die Struktur von reinem, $\mathrm{H}_{2^{-}} / \mathrm{D}_{2}$-markierten $\mathrm{H}^{+}\left(\mathrm{H}_{2} \mathrm{O}\right)_{21}$ im Schwingungsbereich zwischen 600 und $4000 \mathrm{~cm}^{-1}$ 28. Die gefundene Struktur weist ein Hydroniumion auf, dass außen am Cluster gebunden ist (vgl. Abb. 4.4). Die $\mathrm{H}_{2}$ - und $\mathrm{D}_{2}$-Anlagerung dient der Kühlung der erzeugten kationischen Cluster, da durch nachfolgendes Abdampfen die innere Energie verringert wird 27]. Das tagging findet für die selektierten Cluster mit Hilfe von Gaspulsen $\left(10 \% \mathrm{D}_{2}\right.$ in $\left.\mathrm{He}\right)$ in einer Quadrupol-Kühlfalle statt, in deren Inneren eine Temperatur von $6 \mathrm{~K}$ vorliegt 28]. Der Vergleich mit der IR-Absorption natriumdotierter Cluster ist in Abbildung 4.4 enthalten. Für die natriumdotierten Cluster wurden die gemittelten Rohdaten aus neun Messreihen mit Neon als Expansionsgas aufgetragen (die in Tabelle A.2 auf S.VI kursiv markierten Neon-Messtage wurden nicht berücksichtigt). Erst durch das tagging werden mehrere Peaks im Spektrum der kationischen Spezies im Bereich der gebundenen OH-Oszillatoren sichtbar. Die IR-Absorption weist jedoch Unterschiede zu der der natriumdotierten Cluster auf. Auch die in der Publikation berechneten Bandenpositionen stimmen im OHStreckbereich nicht überein. Ähnlichkeiten im Absorptionsverlauf sind zwischen $3200-3500 \mathrm{~cm}^{-1} \mathrm{zu}$ beobachten, was auf ein Vorhandensein des Dodekaeders mit zentralem Wassermolekül im $\mathrm{Na}\left(\mathrm{H}_{2} \mathrm{O}\right)_{21}$-Cluster deuten könnte. Diese Struktur wurde für $\left(\mathrm{H}_{2} \mathrm{O}\right)_{21}$ als mögliche Minimumstruktur von Hartke vorgeschlagen [73. O'Brien et al. haben IR-Spektren für $\mathrm{M}\left(\mathrm{H}_{2} \mathrm{O}\right)_{250 \pm 5}$ mit unterschiedlichen Ionen $\left(\mathrm{M}=\mathrm{SO}_{4}^{2-}, \mathrm{I}^{-}, \mathrm{Na}^{+}, \mathrm{Ca}^{2+}\right.$ und $\left.\mathrm{La}^{3+}\right)$ veröffentlicht und sind damit zum ersten mal größenselektiv in diesen Bereich vorgedrungen [26]. Im Folgenden soll die dieser Studie zugrundeliegende experimentelle Methode vorgestellt werden, da sie, bezügliche der Molekülanzahl, die größten massenselektierten IRPD-Spektren liefert. 


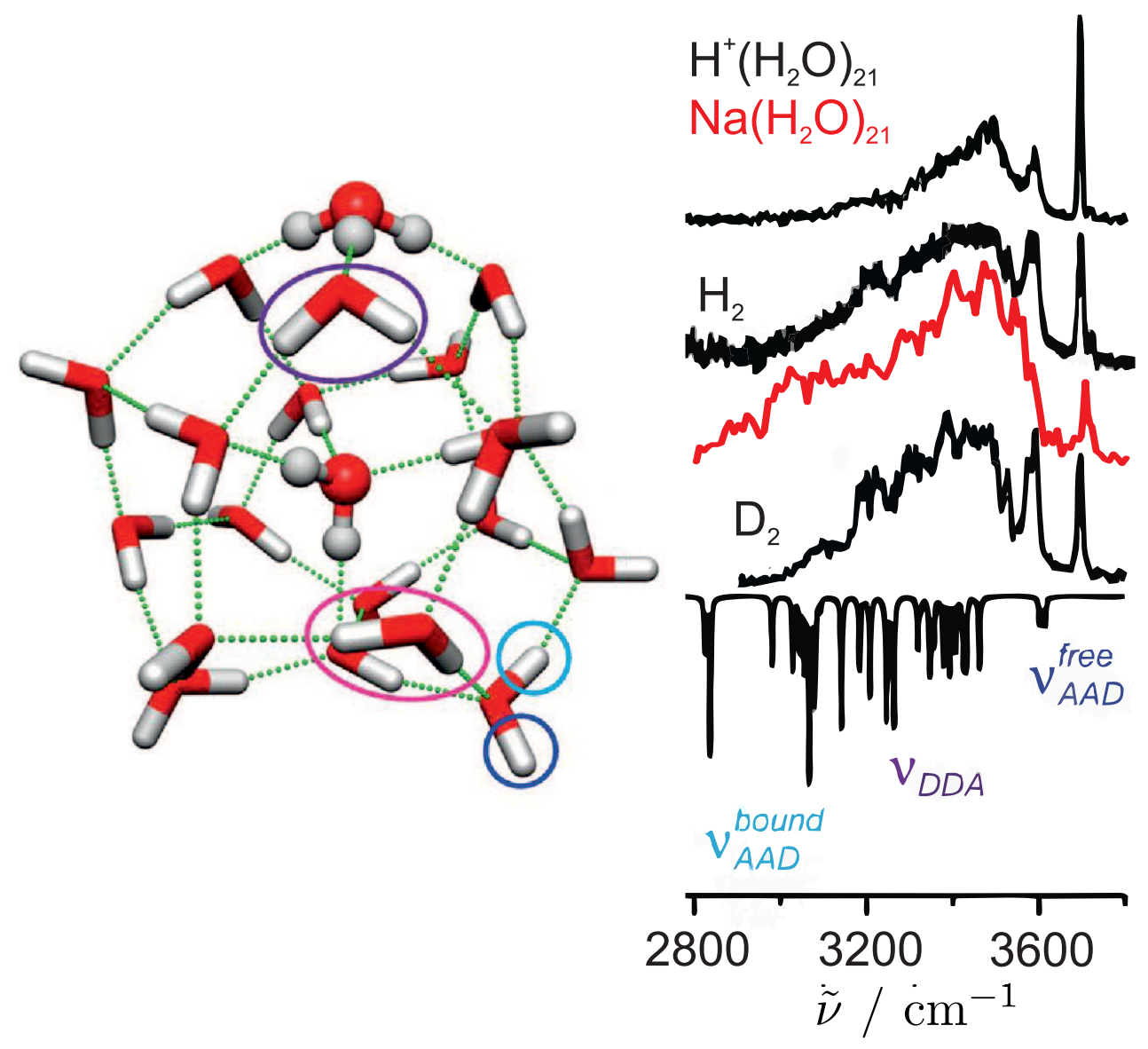

Abbildung 4.4.: Vergleich zwischen simulierten und experimentellen Spektren von $\mathrm{H}^{+}\left(\mathrm{H}_{2} \mathrm{O}\right)_{21}$ (schwarz) und $\mathrm{Na}\left(\mathrm{H}_{2} \mathrm{O}\right)_{21}$ (rot). Die Simulation erfolgte anharmonisch auf dem B3LYP/6-31+G(d) Niveau. Die Struktur ist links abgebildet. Die experimentellen Spektren von reinen $\mathrm{H}_{2}$ und $\mathrm{D}_{2}$-makierten- $\mathrm{H}^{+}\left(\mathrm{H}_{2} \mathrm{O}\right)_{21}$ Clustern wurden mittels IRMPD erhalten. Das $\mathrm{Na}\left(\mathrm{H}_{2} \mathrm{O}\right)_{21}$-Spektrum stellt die gemittelten Rohdaten aus neun Neon-Messungen dar. Weitere Erläuterungen im Text. Die Graphik wurde aus |28| adaptiert.

\subsubsection{Experimentelle Methode}

Die Erzeugung der Cluster erfolgt mittels Nanoelektrospray-Ionisation einer 2 bis $6 \mathrm{mM}$ wässrigen Lösung. Die erzeugten Ionen werden über fünf differentielle Pumpstufen in eine mit Kupfer ummantelte ICR-Zelle geleitet, die auf $133 \mathrm{~K}$ temperiert wird. Die Interaktionszeit in dieser Zelle beträgt 3 bis $6 \mathrm{~s}$ 26. Die Massenseparierung erfolgt mit Hilfe eines 7,0 T starken, supraleitenden Magne- 
ten mittels Fouriertransformations-Ionencyclotronresonanz-Massenspektrometrie (FT-ICR). Die Bestrahlung mit dem Infrarotlaser führt zu einer erhöhten Dissoziationsrate der jeweiligen Clustergröße. Eine detaillierte Beschreibung des experimentellen Aufbaus mit einem 2,75 T starken Magneten ist in der Publikation von Bush et al. zu finden 148.

\subsubsection{Vergleich der IR-Spektren von kationischen und neutralen natriumdotierten Wasserclustern der Größe $n=250$}

Die IR-Spektren von neutralen und ionischen, natriumdotierten Clustern sind in Abbildung 4.5 dargestellt. Für die neutralen Cluster wurden die Größenbereiche $n=225 \pm 25$ und $275 \pm 25$ ausgewählt. Die IR-Spektren stammen aus den gemittelte Daten der Tage 15.03.2012, 21.03.2012, 22.03.2012 und 27.03.2012, die jeweils mit den gleichen Expansionsbedingungen und gleicher Ionisationswellenlänge aufgenommen wurden (vgl. Tabelle A.2 in Kapitel A.3). Die IR-anregungsbedingten Signalzunahmen eines jeden Spektrums wurden durch die maximale Zunahme geteilt. Anschließend wurde der Mittelwert der vier Messreihen für die Clustergrößen $n=225 \pm 25$ und $275 \pm 25$ gebildet. Alle drei Spektren zeigen eine breite Absorption mit ähnlichen Bandenstrukturen zwischen $3150 \mathrm{~cm}^{-1}$ und $3500 \mathrm{~cm}^{-1}$, lediglich die freie OH-Streckschwingung ist separiert. Die neutralen Cluster zeigen dabei ähnliche Absorptionsmuster bei $2850 \mathrm{~cm}^{-1}, 2900 \mathrm{~cm}^{-1}, 2950 \mathrm{~cm}^{-1}, 3020 \mathrm{~cm}^{-1}$ und $3070 \mathrm{~cm}^{-1}$, wobei die Clustergröße $n=275 \pm 25$ markantere Peaks auszubilden scheint. Ab $3050 \mathrm{~cm}^{-1}$ steigt die Absorption der kationischen Cluster an und zeigt mit den neutralen Clustern konforme Muster um $3250 \mathrm{~cm}^{-1}, 3400 \mathrm{~cm}^{-1}, 3500 \mathrm{~cm}^{-1}$ und $3550 \mathrm{~cm}^{-1}$. Das Absorptionsmaximum für $n=225 \pm 25$ und $n=250 \pm 5$ liegt bei $3400 \mathrm{~cm}^{-1}$. Die Clustergröße $n=275 \pm 25$ weist das Maximum bei $3300 \mathrm{~cm}^{-1}$ auf. Der Peak der freien OH-Streckschwingung liegt bei den drei Größenbereichen bei $3698 \mathrm{~cm}^{-1}$ mit einer Schulter zu höheren Wellenzahlen. Unter Berücksichtigung des Signal-Rausch-Verhältnisses weisen $n=225 \pm 25$ und $n=250 \pm 5$ fast identische Spektren auf. Die Clustergröße $n=275 \pm 25$ scheint markantere Peaks auszubilden und hat, im Gegensatz zu den anderen Fällen, ein Absorptionsmaximum bei $3300 \mathrm{~cm}^{-1}$, was auf das Einsetzen der Kristallisation hindeutet (vgl. Kapitel 5). Zusammenfassend lässt sich feststellen, dass die Spektren von O'Brien et al. für 


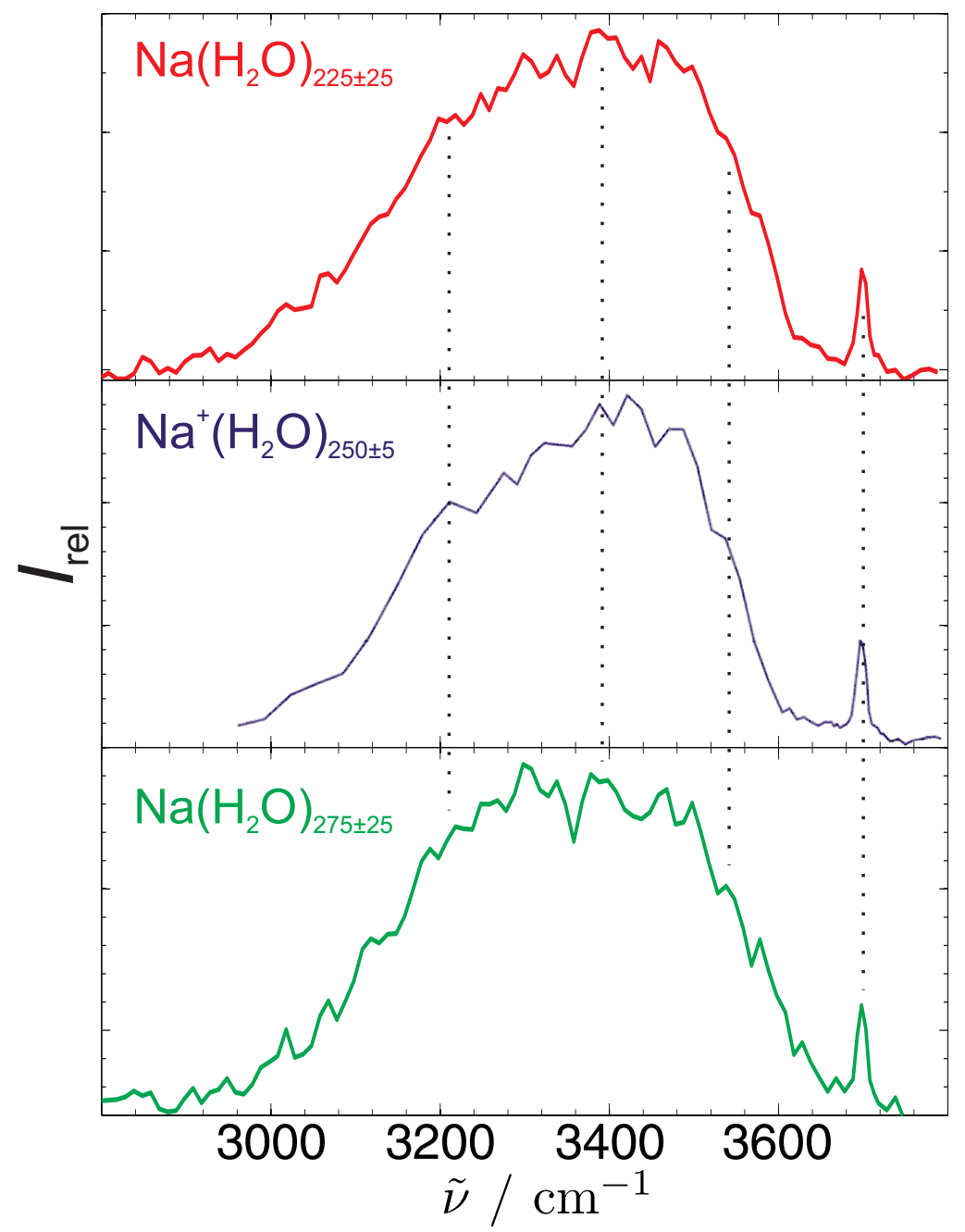

Abbildung 4.5.: Vergleich der IR-Spektren von neutralen und ionischen [26], natriumdotierten Clustern um den Größenbereich von $\bar{n}=250$.

$\mathrm{Na}^{+}\left(\mathrm{H}_{2} \mathrm{O}\right)_{250 \pm 5}$ gut mit denen der neutralen Cluster von $\mathrm{Na}\left(\mathrm{H}_{2} \mathrm{O}\right)_{225 \pm 25}$ übereinstimmen. Bereits in ihrer Publikation wurde die Ähnlichkeit der Spektren mit $\mathrm{I}^{-}\left(\mathrm{H}_{2} \mathrm{O}\right)_{250 \pm 5}$ gezeigt, sodass festgestellt werden kann, dass monovalente Ionen bei dieser Clustergröße nur eine Solvathülle ausbilden und somit aufgrund der marginalen Störung ähnliche Wasserstoffbrückentopologien wie neutrale Cluster formen. Diese Interpretation von O'Brien et al. mit Bezug auf neutrale System konnte erst durch die hier verwendete experimentelle Methode verifiziert werden. Die Abweichungen zu dem nächstgrößeren Clusterbereich sind deutlicher und durch das Einsetzen der Kristallisation bei diesen Expansionsbedingungen (vgl. Kap. 5) und 
die damit einhergehende Umorientierung der Oszillatoren begründet 47].

\subsection{Direkte Absorption großer Partikel}

Die bisher aufgeführten massenselektiven Techniken haben die Auswirkung der Absorption des IR-Lichts auf eine Spezies untersucht. Dies wird als Wirkungsspektroskopie (action spectroscopy) bezeichnet. Die für das Verfahren nötige Massenselektion ist begrenzt durch das Auflösevermögen der verwendeten Massenspektrometer (vgl. Abschnitt 2.3.2). Hüllstromzellen bieten die Möglichkeit große Wasserpartikel mit einem mittleren Durchmesser von bis zu $150 \mathrm{~nm}$ zu erzeugen und die direkte Absorption der Infrarotstrahlung mittels FTIR-Spektroskopie zu messen [43,44]. Eine weitere experimentelle FTIR-Methode im Größenbereich zwischen 3,2 und 5,8 nm wurde von der Gruppe von Wyslouzil 149 entwickelt. Sie kombiniert drei Techniken, um die Nukleation von Wasseraggregaten ortsaufgelöst in gepulsten Lavaldüsenexpansionen im Temperaturbereich zwischen $202 \mathrm{~K}$ und $215 \mathrm{~K}$ zu untersuchen. Zuerst wird das Druckprofil der verwendeten Flussraten bestimmt, wodurch auch Temperatur und Strahlgeschwingkeit zugänglich sind. Mit Hilfe der Röntgenkleinwinkelstreuung (small-angle $\boldsymbol{X}$-ray scattering, SAXS) werden der mittlere Durchmesser und die Teilchendichte ermittelt und anschließend über FTIR der Aggregatzustand der Partikel untersucht. Im Folgenden soll die IR-Spektroskopie an Hüllstromzellen näher erläutert werden.

\subsubsection{FTIR-Spektroskopie an Hüllstromzellen}

Die IR-Spektren der Studie von Medcraft et al. [43] wurden mit Hilfe eines Bruker IFS125/HR Spektrometers und eines Nicolet Magna 550 FTIR Spektrometers aufgenommen und umfassen den Spektralbereich von 10 bis $8000 \mathrm{~cm}^{-1}$. Für die Erzeugung der Aggregate wird zunächst eine Vakuumkammer mit Wasser auf etwa $50{ }^{\circ} \mathrm{C}$ temperiert und ein Druck von 3 bar mit den Gasen Helium oder Stickstoff aufgebaut. Über einen eine Sekunde dauernden Puls bei einem Stagnationsdruck von 2.7 bar gelangt dieses Gemisch in die mit den Trägergasen Helium oder Stickstoff vortemperierte Hüllstromzelle. Über inelastische Stöße werden die Wassermoleküle abgekühlt, bis die Gasphase übersättigt ist. Anschließend beginnt die Nukleation der Partikel. Die Studie umfasst einen Größenbereich von 3 bis 100 nm und Tempe- 
raturbereich von 5 bis 209 K. Für die Temperierung wird je nach Bereich flüssiges oder gasförmiges Helium bzw. flüssiger oder gasförmiger Stickstoff verwendet. Die Konzentration des Wassers in der Zelle wird auf 0,1 bis $1 \%$ geschätzt.

\subsubsection{Vergleich der IR-Spektren natriumdotierter Cluster mit IR-Absorptionsspektren von Wasseraggregaten}

Der Vergleich eines IR-Spektrums von natriumdotierten Wasserclustern der Größe $n=575 \pm 25$ mit den IR-Spektren verschiedener Größenbereiche der im vorherigen Abschnitt erläuterten Methode ist in Abbildung 4.6 dargestellt. Die FTIRSpektren wurden bei einer Temperatur von $78 \mathrm{~K}$ aufgenommen. Die Temperatur der natriumdotierten Cluster bei diesen Expansionsbedingungen wurde in der $\mathrm{Pu}$ blikation von Buck et al. auf $50 \mathrm{~K}$ abgeschätzt [11]. Aus Messungen des PickupQuerschnitts von Lengyel et al. lässt sich ein Durchmesser von 4 bis 5,5 nm für die Clustergröße $n=575 \pm 25$ abschätzen $[150 \mid$. Der geometrische Durchmesser sollte weniger als $4 \mathrm{~nm}$ betragen.

Für die Erzeugung der IR-Spektren der natriumdotierten Cluster wurden als Expansionsbedingungen eine Ofentemperatur von $\vartheta_{\text {Ofen }}=140^{\circ} \mathrm{C}$ eingestellt und Helium als Trägergas bei einem Stagnationsdruck von 4,5 bar verwendet. Die Ionisationswellenlänge betrug $390 \mathrm{~nm}$ (vgl. Tabelle A.2 im Anhang). Das Spektrum ist 10 mal geglättet. Zusätzlich erfolgt noch eine Gegenüberstellung mit einem von Buch et al. [44] berechneten IR-Spektrum der Clustergröße $n=600$, dem ein Isomer zu Grunde liegt, dessen Geometrieoptimierung aufgrund der Größe mit Hilfe des effizienten TIP4P-Potentials [116] erfolgte. Die Einzelheiten zur Simulation des IR-Spektrums können der Publikation von Sadlej et al. [151] entnommen werden. Die markanteste Übereinstimmung ist der Peak bei $3220 \mathrm{~cm}^{-1}$, der dem kristallinen Kern der Partikel zugeschrieben werden kann. Die Maximumsposition ist von Größe und Temperatur der Cluster abhängig. Je größer oder kälter ein Cluster ist, desto weiter wandert der Peak zu kleineren Wellenzahlen, deutlich zu erkennen in der Gegenüberstellung der verschiedenen Größenbereiche der FTIR-Spektren. Die beiden im simulierten Spektrum vorhergesagten Schultern bei $3000 \mathrm{~cm}^{-1}$ und $3100 \mathrm{~cm}^{-1}$, die von oberflächennahen Oszillatoren stammen, werden im größenselektiv gemessenen Spektrum beobachtet. Bei den direkten Absorptionsmessungen über die gesamte Größenverteilung tritt die zweite Schulter 


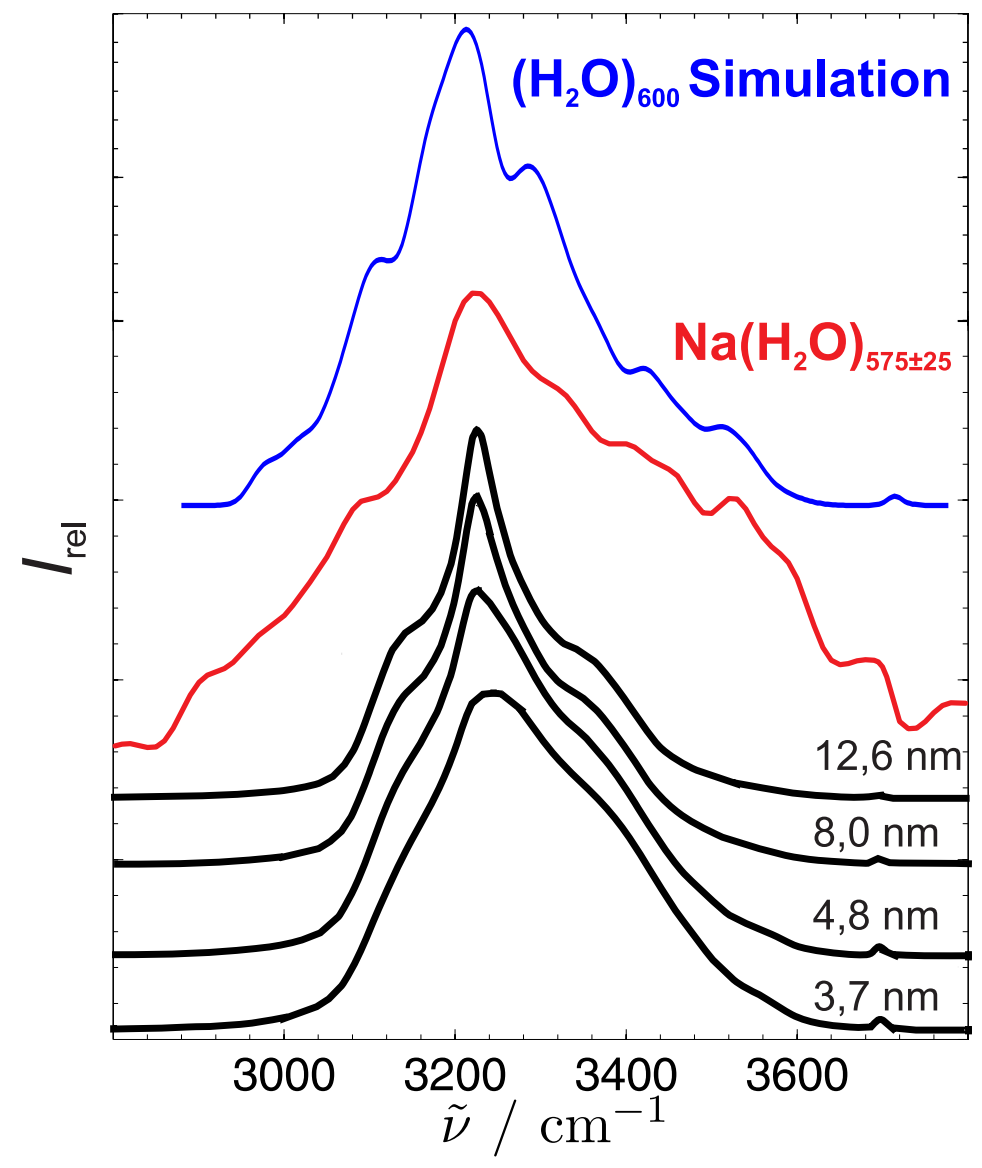

Abbildung 4.6.: Vergleich des IR-Spektrums vom natriumdotierten Wasserclustern der Größe $n=575 \pm 25$ mit FTIR-Spektren von 3,7 bis $12,6 \mathrm{~nm}$ großen Partikeln [43] und einem berechneten Spektrum der Größe $n=600$ 44.

erst bei einem durchschnittlichen Partikeldurchmesser von 8,0 nm $(\mathrm{n} \approx 5400)$ auf. Die OH-Oszillatoren der Oberfläche, deren Absorption hauptsächlich oberhalb von $3220 \mathrm{~cm}^{-1}$ liegt, zeigen bei allen drei Methoden ein unterschiedliches Verhalten. Der Peak bei $3400 \mathrm{~cm}^{-1}$ und die Schulter bei $3500 \mathrm{~cm}^{-1}$ im größenselektiven Fall stimmen mit den simulierten Spektren von Buch überein. Im FTIR-Spektrum für $3,7 \mathrm{~nm}$ große Partikel ist hier nur eine breite Absorption zu erkennen. Die relativ zum Maximum bei $3220 \mathrm{~cm}^{-1}$ erhöhte Intensität im experimentellen Spektrum der $\mathrm{Na}\left(\mathrm{H}_{2} \mathrm{O}\right)_{575 \pm 25}$-Cluster könnte auf eine höhere Sensitivität des genutzten Wirkungseffekts der IR-Strahlung auf Oberflächenoszillatoren zurückzuführen sein. Diese Erklärung passt auch zu der im Vergleich zu den FTIR-Spektren hohen In- 
tensität der freien OH-Streckschwingung, die ebenfalls in den Spektren der Gruppen von Fujii und Williams eine hohe relative Intensität aufweist. Unterschiede zu den FTIR-Spektren sind vermutlich auf den genutzten Wirkungseffekt zurückzuführen.

Das Massenspektrum der natriumdotierten Cluster für die gewählten Expansionsbedingungen ist in Abbildung $4.7 \mathrm{zu}$ finden. Für eine bessere Anschaulichkeit

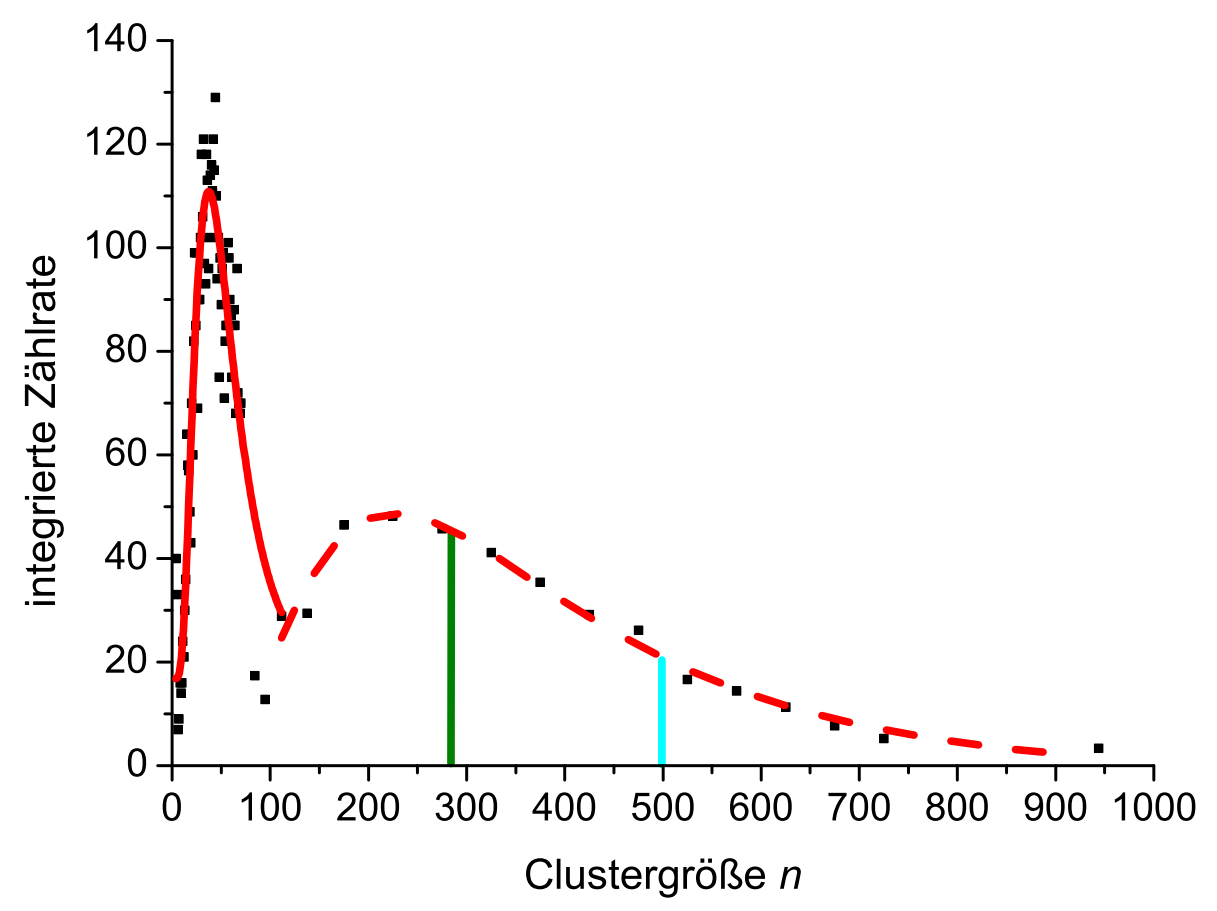

Abbildung 4.7.: Integrierten Zählraten des Massenspektrums der natriumdotierten Cluster bei einer Ionisationswellenlänge von $390 \mathrm{~nm}$ bei einem Stagnationsdruck von 4,5 bar. Weitere Erläuterungen sind im Text zu finden.

wurden die integrierten Zählraten aufgetragen. Da zwei Auswertungsroutinen verwendet wurden, eine für die einzelnen Clustergrößen bis $n=70$ und eine für unterschiedliche Intervalle ab $n \geq 78$, erfolgte eine Anpassung der Zählrate der Intervalle 
mit Hilfe der Formel1:

$$
\text { integrierte Zählrate } n=\frac{\text { integrierte Zählrate des Intervalls }(n \pm \Delta n)}{\sqrt{2 \Delta n}}
$$

Die roten Graphen entsprechen zwei Log-Normalverteilungen, eine für den Clustergrößenbereich (durchgezogene Linie) und eine für die nach obiger Formel angepassten Zählraten (gestrichelte Linie) der Intervalle. Etwa 10\% der am MCP registrierten Teilchen sind kristallin. Sie treten erst ab einer Clustergröße von $n=525 \pm 25$ auf. Eine detailliertere Beschreibung des Vorgangs der größenselektiven Kristallisation von Wasserclustern ist im folgenden Kapitel 5 zu finden. Die

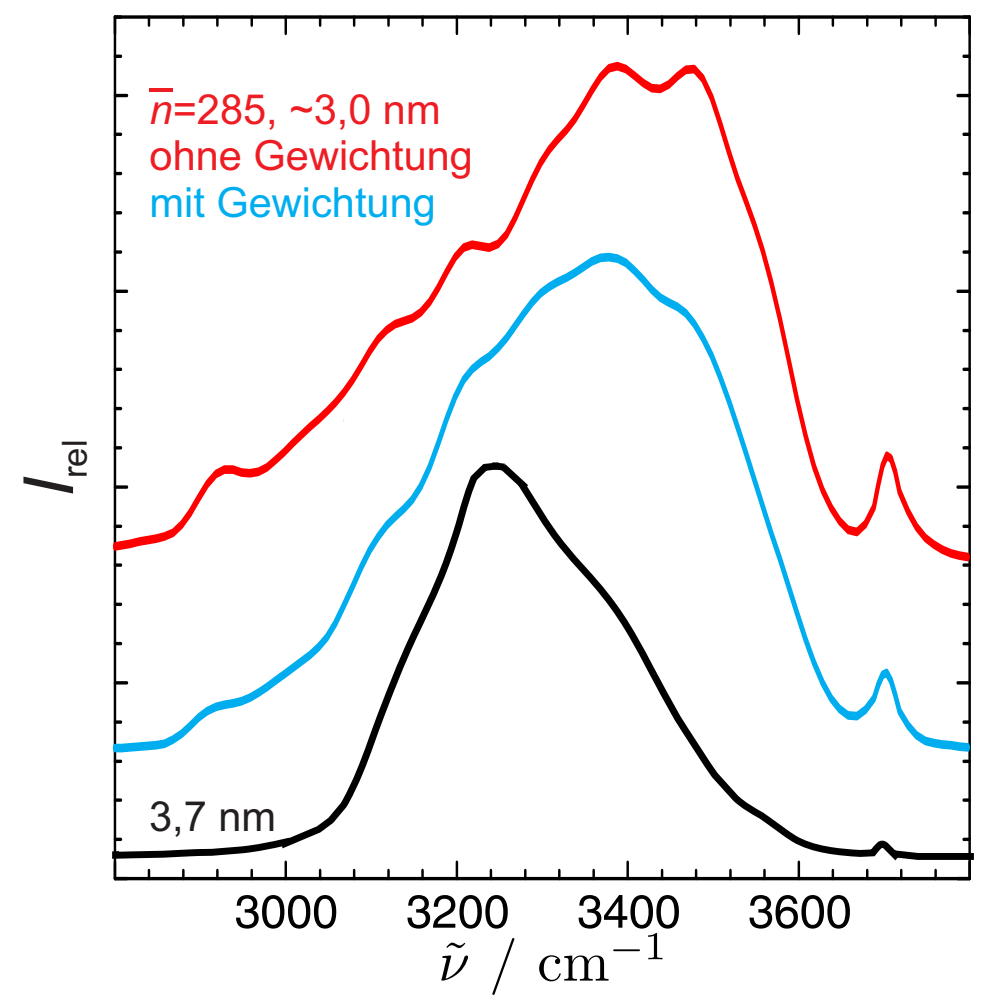

Abbildung 4.8.: Vergleich der Gesamtabsorption natriumdotierter Cluster der Messreihen vom 11. und 13.04.2012 (Expansionsparameter in Tabelle A.2 im Anhang) mit einem FTIR-Spektrum von im Durchmesser 3,7 nm großen Partikeln [43]. Erläuterungen im Text.

Massenverteilung hat eine durchschnittliche Clustergröße von $\bar{n}=285$, was einem

\footnotetext{
${ }^{1}$ Aufgrund von Skalierungsgründen wird für die Anpassung der Faktor $\sqrt{2 \Delta n}$ im Gegenteil zum korrekten Faktor $(2 \Delta n)$ verwendet.
} 
Durchmesser von ungefähr $3 \mathrm{~nm}$ entsprechen würde [149]. Der Mittelwert der Clusterverteilung ist in Abbildung 4.7 durch die grüne Linie gekennzeichnet und wurde direkt aus den Zählraten der Massenspektren bestimmt. Er stimmt gut mit dem Wert aus der empirischen Formel 2.4 auf Seite 8 aus der Arbeit von Bobbert et al. [32 überein. Diese liefert mit den Ausgangsparametern der Expansion $p_{0}$ als Stagnationsdruck und $T_{0}$ als Reservoirtemperatur die mittlere Clustergröße von $\bar{N}=297$ für Expansionen ohne Trägergas. Der Unterschied kann durch die elliptische Düse bedingt sein, deren Öffnungsdurchmesser zwischen $60 \mu \mathrm{m}$ und $70 \mu \mathrm{m}$ liegt 46. Der geringe Anteil an kristallinen Strukturen im Clusterstrahl führt, wenn man die Gesamtabsorption der Verteilung betrachtet (s. Abb. 4.8), zu einem Absorptionsmuster, das amorphen Clustern zugeordnet werden kann. Dabei wurde die Gesamtabsorption auf zwei unterschiedliche Weisen für die Messtage am 11. und 13.04.2012 gebildet. Zum einen wurden die normierten Zählraten eines jeden Massenspektrums aufsummiert und anschließend das IR-Spektrum erzeugt. Da jedoch ein Unterschied zwischen der IR-Laserinduzierten Zunahme und der Absorption von Licht einer Clusterverteilung besteht, wurde zusätzlich ein Spektrum gebildet, das mit einem Gewichtungsfaktor diesen Unterschied berücksichtigen soll. Hierfür wurden alle erhalten größenselektiven IR-Spekten zunächst auf den Maximalwert 1 normiert und gewichtet nach ihrer relativen Häufigkeit in der Massenverteilung sowie der Clustergröße. Die Annahme ist hier, dass jeder gebundene Oszillator im gleichen Maße Licht absorbiert. Für den Vergleich wurden die Spektren der beiden Messtage auf identische maximale Intensität normiert und der Mittelwert gebildet. In Abbildung 4.8 sind die natriumdotierten Spektren zehnfach geglättet im Vergleich zu der Absorption von 3,7 nm großen Partikeln in rot bzw. blau dargestellt. Das Maximum der Absorption ist für die natriumdotierten Spektren bei $3400 \mathrm{~cm}^{-1}$. Es werden breite Peaks beim Spektrum ohne Gewichtung bei ungefähr $2900 \mathrm{~cm}^{-1}, 3200 \mathrm{~cm}^{-1}, 3500 \mathrm{~cm}^{-1}$ und $3700 \mathrm{~cm}^{-1}$ ausgebildet. Diese Oszillatoren können der Oberfläche der Cluster zugeordnet werden. Die Gewichtung erzeugt ebenfalls ein amorphes Spektum mit höherer relativer Absorption um $3200 \mathrm{~cm}^{-1}$ und einer verringerten relativen Absorption der freie OH-Streckschwingung.

Die IR-CIPI-Methode liefert in dem Größenbereich um $4 \mathrm{~nm}$ IR-Spektren, die mit FTIR-Messungen schwer vergleichbar sind, aber gute Übereinstimmung mit simulierten Spektren zeigen. Aufgrund der Größenselektivität können von Buch et 
al. vorhergesagte spektrale Absorptionsmuster 44 der Kristallisation von Wasserpartikeln auf Clusterebene im Größenbereich von $n=550$ bis $n=600$ beobachtet werden. Die Effizienz des Wirkungseffekts bei den vorliegenden experimentellen Bedingungen scheint für oberflächennahe Oszillatoren größer zu sein. 


\section{Kristallisation von Wasserclustern}

Wie viele Wassermolekülen werden benötigt, um ein Kristallgitter wie in Eis zu formen? Das ist eine spannende Fragestellung, deren Klärung das Verständnis der Wasserstoffbrückeninteraktionen, welche den makroskopischen Eigenschaften von Wasser zugrundeliegen, fördert. Allerdings gestaltet sich die experimentelle Realisierung schwierig, da sie zwei Voraussetzungen erfüllen muss. Die Erste ist die Größenselektivität, die durch Kopplung des Messverfahrens an ein Massenspektrometer realisiert werden kann. Als zweites muss das Verfahren zwischen kristallinen und nicht-kristallinen Clustern unterscheiden können. Die in Kapitel 2.3.1.2 vorgestellte Methode erfüllt diese beiden Voraussetzungen. Betrachtet man das IR-Spektrum in Abbildung 5.1, so erkennt man eine klare Separierung der Absorptionsmaxima zwischen gasförmigem, flüssigem und kristallinem Wasser. Während die Gasphase bei $25^{\circ} \mathrm{C}$ spezifische Peaks zwischen $3500 \mathrm{~cm}^{-1}$ und $4000 \mathrm{~cm}^{-1}$ aufweist, ist bei flüssigem Wasser nur eine breite Absorption zwischen $3100 \mathrm{~cm}^{-1}$ und $3700 \mathrm{~cm}^{-1}$ mit einem Maximum bei $3400 \mathrm{~cm}^{-1}$ vorhanden. Das IR-Spektrum von Eis besitzt, wie bereits in Kapitel 4.3.1 beschrieben, einen ausgeprägten Peak bei $3220 \mathrm{~cm}^{-1}$ mit charakteristischen Schultern bei $3150 \mathrm{~cm}^{-1}$ und $3350 \mathrm{~cm}^{-1}$.

Ein Nachteil der IR-Spektroskopie ist, dass sie nicht leicht zwischen kubischem $\left(\mathrm{I}_{\mathrm{c}}\right)$ und hexagonalem $\left(\mathrm{I}_{\mathrm{h}}\right)$ Eis unterscheiden kann 83. Aus Elektronen- und Röntgenbeugungsexperimenten von Stein [153], Bartell [154, 155] und Murray et al. 156, 157 ist bekannt, dass kalte Wassernanotröpfchen zu kubischem Eis $\mathrm{I}_{\mathrm{c}}$ und nicht zum (makroskopisch) thermodynamisch stabileren, hexagonalen Eis $\mathrm{I}_{\mathrm{h}}$ gefrieren. Torchet und Mitarbeiter konnten in Elektronenbeugungsexperimenten an im Molekularstrahl erzeugten Wasserclustern den Größenbereich für das Einsetzen der Kristallisation auf $n=200-1000$ abschätzen 45. 


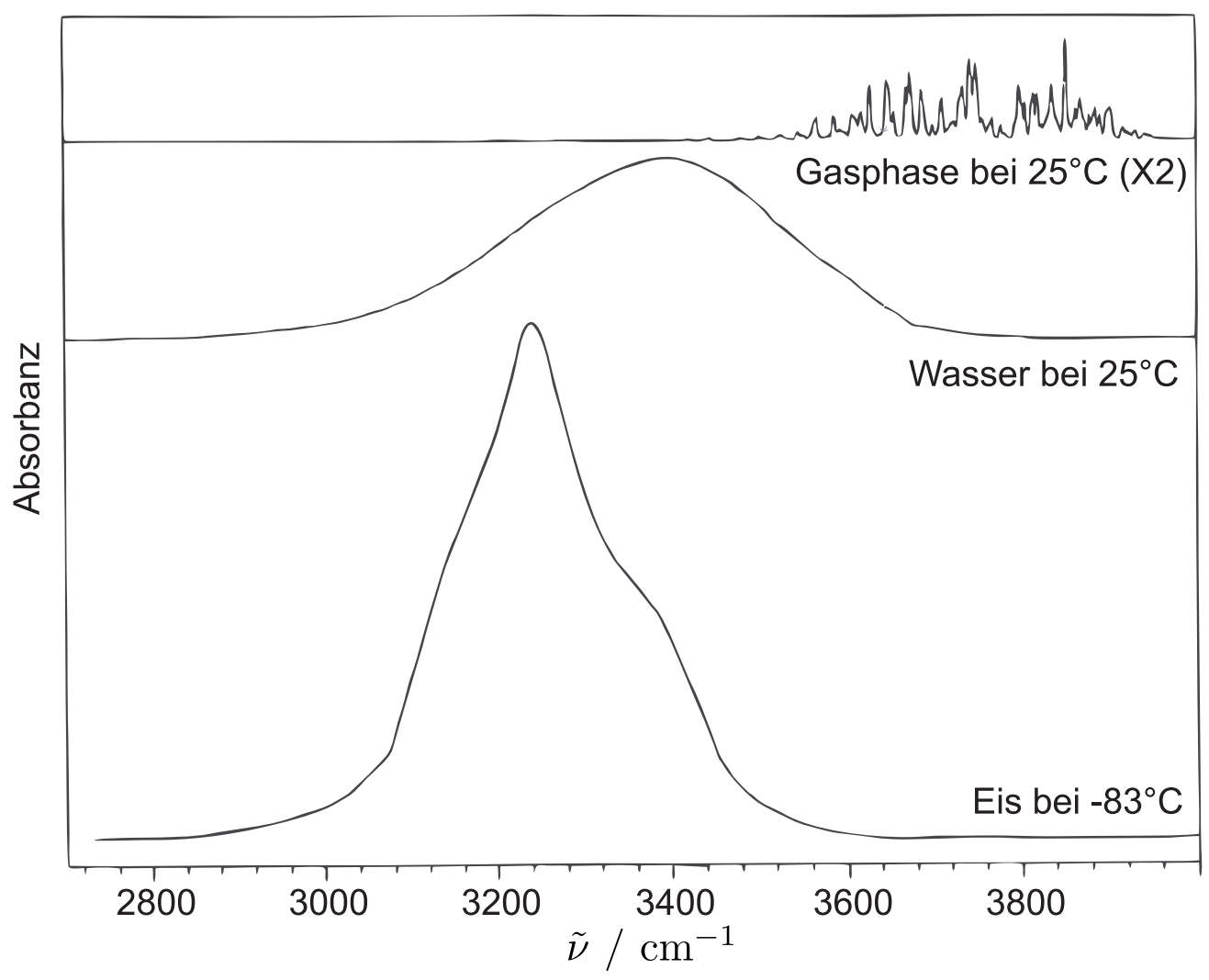

Abbildung 5.1.: Infrarot-Spektrum von Wasser in verschiedenen Aggragatzuständen. Die Absorption wurde auf die gleiche Anzahl an Wassermolekülen im Lichtweg skaliert. Im Fall vom gasförmigen Wasser wurde sie zusätzlich mit dem Faktor 2 multipliziert. Verändert nach 152 .

Im Folgenden sollen zunächst die von Buch et al. für den Übergangsbereich amorph-kristallin simulierten Spektren von Wasserclustern vorgestellt werden [44]. Anschließend werden die experimentellen, mit der IR-CIPI-Methode bei unterschiedlichen Expansionsbedingungen erzeugten Spektren betrachtet.

\subsection{Simulation größenselektiver IR-Spektren}

Die Grundlage für jedes simulierte Spektrum sind Strukturen, die sich in einem lokalen Minimum der Potentialhyperfläche (potential energy surface, PES) befinden. Dabei gibt es zahlreiche Möglichkeiten der Anordnung von Molekülen in einem Cluster. In der Studie von Buch et al., die einen Größenbereich von $n=20$ 
bis 931 umfasst, verläuft die Suche der Minimumstrukturen zunächst über MDSimulationen bei unterschiedlichen Anfangstemperaturen (100-200 K), bei denen die Strukturen nach einem definierten Zeitfenster optimiert werden [44]. In dem Temperaturbereich 130-160 K ist dabei die Mobilität der Moleküle groß genug, um die Barrieren zwischen den lokalen Minimumstrukturen zu überwinden und trotzdem die niedrigen Energieregionen zu bevorzugen. Bei höheren Temperaturen werden nur Strukturen mit hohen Energien generiert. In einem zweiten Schritt werden bei den erhaltenen Minimumstrukturen über einen Monte-Carlo-Algorithmus 158 die Positionen der Wasserstoffatome für eine vorgegebene Sauerstoffkonfiguration optimiert. Zuletzt werden mit Hilfe der Rigid Body Diffusion Monte Carlo Methode (RBDMC) [159] die erhaltenen Strukturen nochmals optimiert und der Grundzustand (die Nullpunktsenergie) ermittelt. Als Potential wurde TIP4P [116 und für einige wenige, kleinere Clustergrößen TTM2-R [117 verwendet. Die Minimumstrukturen für $n=293,600$ und 931 wurden aus sphärischen Ausschnitten der bekannten Struktur von Eis $I_{c}$ abgeleitet, bei denen die Oberfläche nach obigem Schema optimiert wurde. Dies führte zu einer Halbierung der Anzahl dreifach koordinierter Wassermoleküle im Verhältnis zur Ausgangsstruktur.

Das IR-Spektrum wurde mit einem anharmonischen Oszillatormodell aus der lokalen Minimumstruktur der Cluster berechnet. Bei der Simulation wurden die $\mathrm{OH}$-Bindungen als lokale Morse-Oszillatoren, die an die inter- und intramolekularen Dipol-Dipol-Wechselwirkungen gekoppelt sind, behandelt. Die Schwingungsfrequenz ist empirisch in Relation zu dem elektrische Feld in Richtung der Wasserstoffbrückenbindung kalibriert. Die Funktion wurde für $\left(\mathrm{H}_{2} \mathrm{O}\right)_{8}$ an die dazugehörigen experimentelle Daten [151], sowie für $\left(\mathrm{H}_{2} \mathrm{O}\right)_{931}$ an experimentelle Daten von Eis 160 angepasst.

In Abbildung 5.2 ist eine Übersicht der berechneten Spektren für $\left(\mathrm{H}_{2} \mathrm{O}\right)_{n}$ mit $n=123,293,600$ und 931 dargestellt. Den Spektren von $n=123$ und 293 liegen jeweils die 10 energetisch günstigsten Isomere im TIP4P-Potential zugrunde. Für $n=600$ und 931 entspricht das Spektrum der Minimumstruktur aus diesem Potential. Neben den Spektren sind die dazugehörigen Strukturen abgebildet, wobei im Fall von $n=293$ und 931 zur besseren Illustration ein Ausschnitt des Clusters dargestellt ist. $\left(\mathrm{H}_{2} \mathrm{O}\right)_{123}$ stellt ein Beispiel für einen amorphen Cluster dar. Das Maximum der Absorption im Spektrum liegt bei $3350 \mathrm{~cm}^{-1}$ und entspricht der $\mathrm{OH}-$ Streckschwingung vierfach koordinierter Moleküle, deren Anteil verglichen mit 


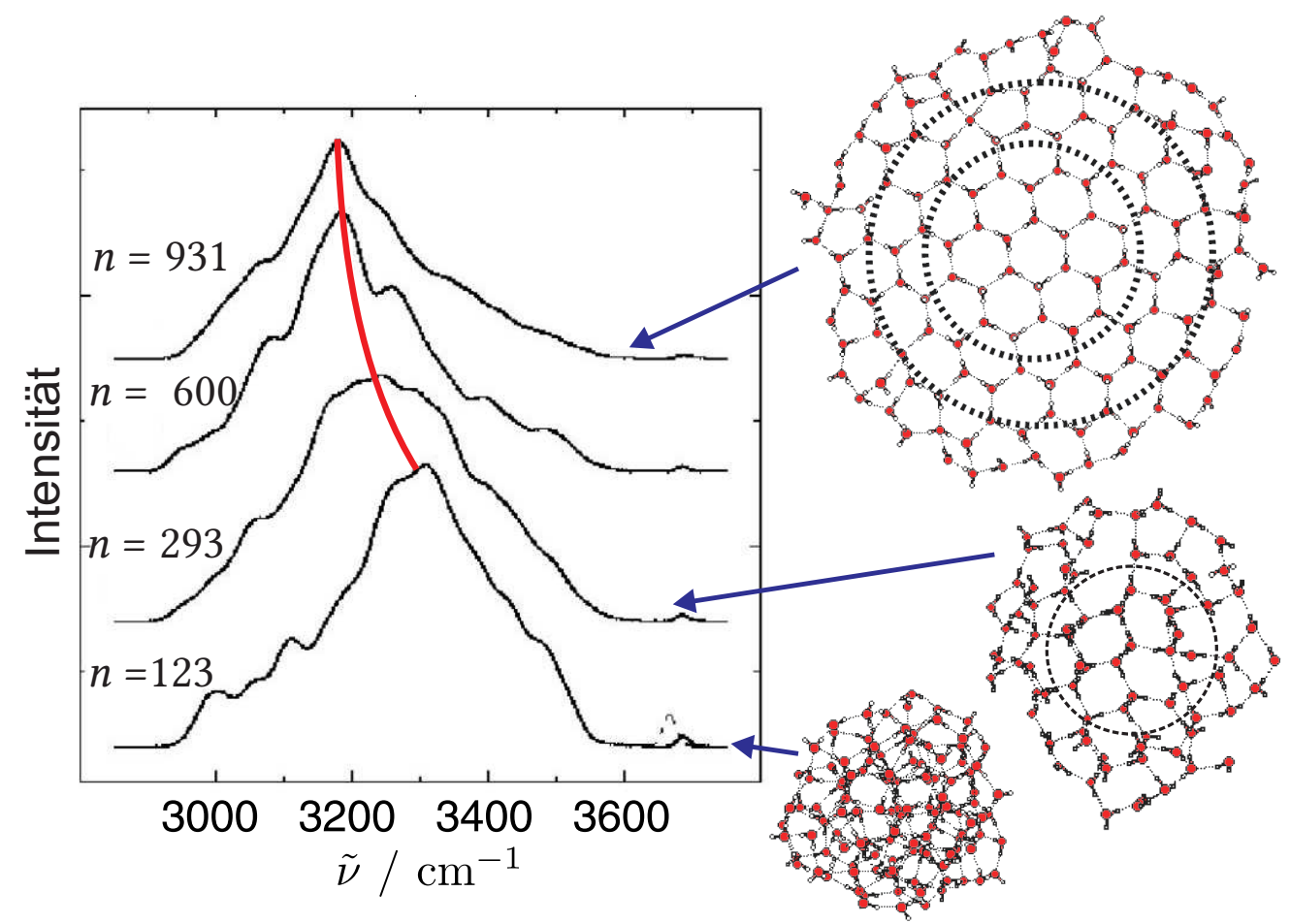

Abbildung 5.2.: Simulierte Spektren und Strukturen der $\left(\mathrm{H}_{2} \mathrm{O}\right)_{n}$-Cluster. Für die Clustergrößen $n=123$ und 293 wurde das Spektrum über die 10 energetisch günstigsten Isomere im TIP4P-Potential gemittelt. Die Spektren für $n=600$ und 931 entsprechen der Minimumstruktur. Für $n=293$ und 931 ist ein Querschnitt durch den Cluster abgebildet. Verändert nach [44.

anderen Bindungsmotiven am höchsten ist. Der Übergang zum kristallinen Cluster findet bei $n=293$ statt. Das Spektrum weist ein im Gegensatz zur vorherigen Größenscheibe breiteres Plateau der Absorption mit amorphen und kristallinen Anteilen auf. Die Kristallisation führt zur Ausbildung einer honigwabenenartigen Struktur von Sechsringen im Inneren der Cluster, angedeutet durch den gestrichelten Kreis für $n=293$ in Abbildung 5.2. Die Kooperativität führt zu einer Rotverschiebung der DDAA-Schwingung zu ca. $3220 \mathrm{~cm}^{-1}$. Die Clustergrößen $\left(\mathrm{H}_{2} \mathrm{O}\right)_{600}$ und $\left(\mathrm{H}_{2} \mathrm{O}\right)_{931}$ entsprechen Strukturen mit hohem kristallinen Anteil. Das Maximum der Absorption liegt bei der kristallinen Bande bei ungefähr $3220 \mathrm{~cm}^{-1}$. Für $n=931$ sind $81 \%$ der Moleküle vierfach koordiniert [44]. Die Lage der Moleküle im Cluster lässt sich in Kern, oberflächennah und Oberfläche einteilen (s. gestrichelte Linien in Abb. 5.2). Die Moleküle des Kerns formen die honigwabenenartigen 
Struktur von Eis $I_{c}$. Die oberflächennahen Moleküle bilden ebenfalls Sechsringe aus, sie grenzen jedoch hier an vier- und fünfgliedrige Ringe, die zur Oberfläche gehören. Die kristalline Bande wird vom Kern und oberflächennahen Oszillatoren ausgebildet, wobei letztere für die Schulter unterhalb von $3200 \mathrm{~cm}^{-1}$ verantwortlich sind. Stärker rotverschobene Absorptionsbanden werden durch die dreifach koordinierten DAA Moleküle der Oberfläche verursacht. Die übrigen Oszillatoren der Oberfläche absorbieren, wie in Tabelle 2.1 auf Seite 12 vermerkt, oberhalb von $3300 \mathrm{~cm}^{-1}$.

\subsection{Experimentelle Spektren}

Die Möglichkeit der Erzeugung und Photoionisation großer, natriumdotierter Wassercluster in der hier eingesetzten Apparatur wurde bereits in den Arbeiten von Bobbert et. al [32] und Forck et al. [60| aufgezeigt. In der ersten Arbeit fand eine systematische Untersuchung der Größenverteilung bei unterschiedlichen Expansionsbedingungen und Düsengeometrien statt. In der Zweiten wurde das Ionisationspotential großer Cluster untersucht und so das Vorhandensein einer zweiten Isomerklasse mit einem geringeren IP bei $2,8 \mathrm{eV}$ ab einer Clustergröße von $n \approx 15$ demonstriert. Die Erzeugung von IR-Spektren mittels IR-anregungsmodulierter Photoionisation wurde in der ersten Phase der vorliegenden Arbeit für Wasserund Methanolcluster bis zu einer Größe von ungefähr $n=60$ [38, 39, 41, 42] bzw. $n=7$ 40,41 untersucht. In Kapitel 4.3.2 wurde bereits gezeigt, dass die IR-CIPI Methode bis zu einem Größenbereich von $n \approx 600$ verwendet werden kann. Die Erzeugung großer Cluster in Überschallexpansionen erfordert hohe Stagnationsdrücke für den Koagulationsprozess. Bei Forck et al. wurde eine reine Wasserexpansion bei 6,5 bar Stagnationsdruck verwendet [41,60|. Um eine bessere Abkühlung zu gewährleisten, werden Trägergase wie Helium, Neon und Argon eingesetzt. Dabei wird die Temperatur der Cluster umso niedriger, desto höher der angelegte Druck ist. Der limitierende Faktor für die Erzeugung ist dabei die Pumpleistung in der Ofenkammer, die für verschiedene Gasmoleküle unterschiedlich ausfällt. Überschreitet der Hintergrunddruck den Bereich von $10^{-4}$ mbar, so führt dies zum Zusammenbruch des Molekularstrahls.

Im Folgenden soll der Vorgang der Kristallisation für unterschiedliche Expansionen 
mit Helium und Neon vorgestellt werden. Die Expansionsparameter wurden dabei

Tabelle 5.1.: Übersicht der Expansionsbedingungen für die Kristallisation der Wassercluster, mit $p$ ist der Stagnationsdruck angegeben. Die Temperaturen wurden der Publikation [11] entnommen. Weitere Erläuterungen im Text.

\begin{tabular}{c||c|c|c|c}
\hline Expansion & Trägergas & $p /$ bar & $T_{\text {Ofen }} / \mathrm{K}$ & $T_{\text {Cluster }} / \mathrm{K}$ \\
\hline \hline A (,kalt“) & $\mathrm{He}$ & 4,5 & 413 & $\sim 50$ \\
B (,medium“) & $\mathrm{He}$ & $3,9 \pm 0,1$ & 403 & $\sim 70$ \\
C (,warm, He“) & $\mathrm{He}$ & 3,5 & 393 & s. Text \\
D (,warm, Ne“) & $\mathrm{Ne}$ & $2,6 \pm 0,3$ & 373 & $\sim 100$ \\
\hline
\end{tabular}

so eingestellt, dass im Betrieb mit dem Pumpstand „Ofenkammer regulär“ (vgl. Tabelle 3.2 auf Seite 42 bei vorgegebener Ofen- und Düsentemperatur der maximal mögliche Stagnationsdruck angelegt wurde. In Tabelle 5.1 wird eine Übersicht der Expansionsbedingungen gegeben und in Abbildung 5.3 ein Ausschnitt aus den dazugehörigen Massenspektren dargestellt. Die in der Tabelle angegebenen Temperaturen stammen aus der Publikation [11] und sind Schätzungen von Udo Buck. Sie sollen daher eher Trends veranschaulichen. Für $\left(\mathrm{H}_{2} \mathrm{O}\right)_{6}$ konnten die von Udo Buck abgeschätzten Temperaturen durch die Simulationen von Tainter und Skinner $\mid 70$, die die Änderung der Isomerenzusammensetzung und ihrer Wirkung auf das IR-Spektrum betrachteten, verifiziert werden. Die Temperatur der als „warm“ deklarierten Heliummessung kann auf Basis der Fälle A und B grob auf ungefähr $90 \mathrm{~K}$ abgeschätzt werden.

\subsubsection{Kristallisation bei Helium als Trägergas}

Betrachtet wird zunächst der Expansionsfall A, der Clustern mit der ,geringsten" Ausgangstemperatur in einer Heliumexpansion erzeugt. Dieser Expansionsfall wurde ebenfalls in Kapitel 4.3.2 für den Vergleich mit den FTIR-Messung an Hüllstromzellen herangezogen. Die größenselektive Entwicklung der IR-Spektren zwischen $85 \pm 5$ und $575 \pm 25$ ist in Abbildung 5.4 zu finden. Den Daten zugrunde liegen zwei Messungen mit einem Stagnationsdruck von 4,5 $\pm 0,1$ bar. Die Düsentemperatur betrug $145^{\circ} \mathrm{C}$ und die Ofentemperatur $140^{\circ} \mathrm{C}$. Die Photoionisation erfolgte bei $390 \mathrm{~nm}$. Die Zunahmen wurden durch den Maximalwert geteilt und 


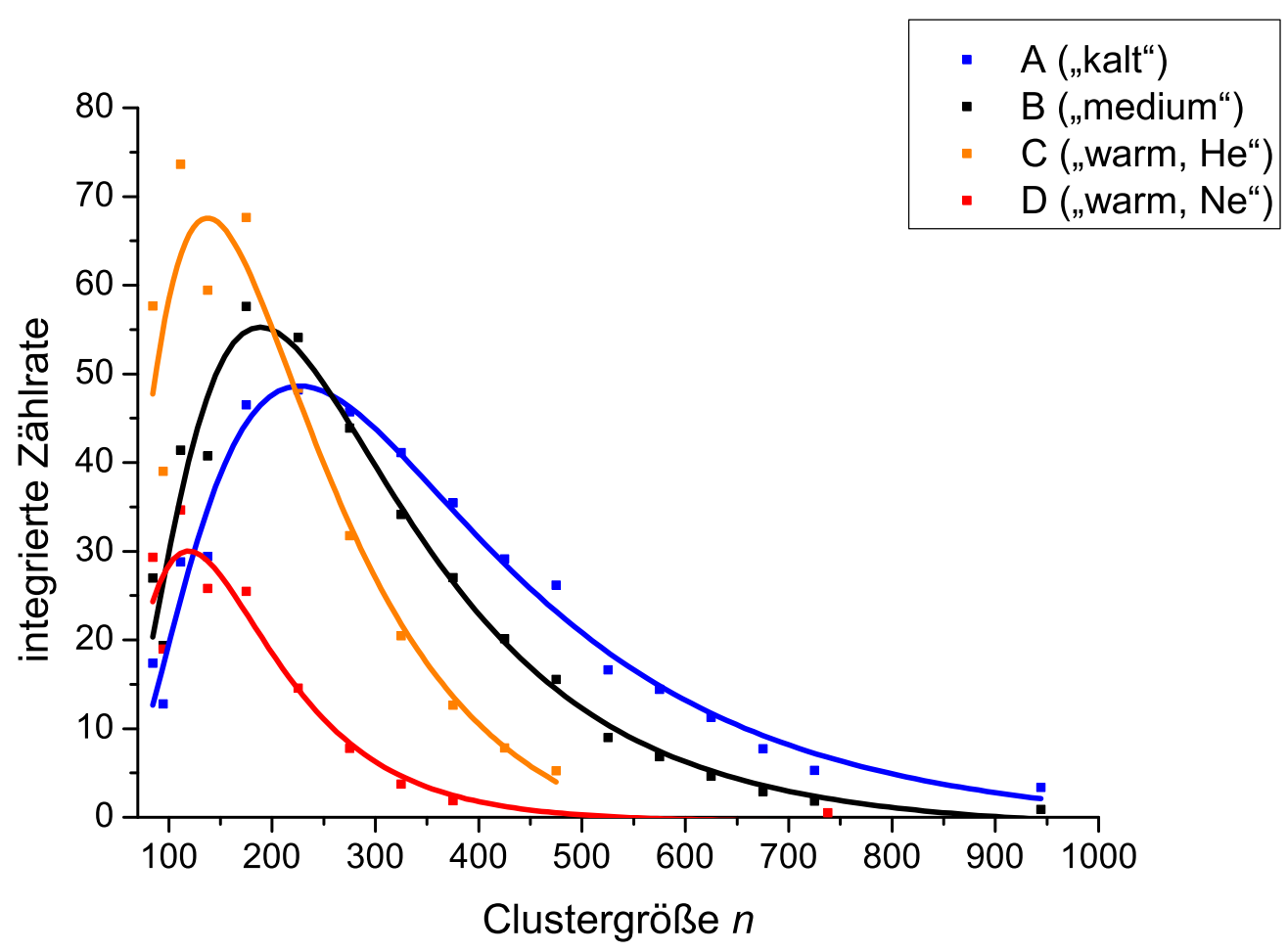

Abbildung 5.3.: Vergleich der integrierten Zählraten der natriumdotierten Cluster bei verschiedenen Expansionsbedingungen, die in Tabelle $5.1 \mathrm{zu}$ finden sind. Die Zählraten wurden nach Gleichung 4.1 auf Seite 83 angepasst.

der Mittelwert aus den Messungen gebildet. Anschließend erfolgte eine zehnfache Dreiecksglättung um das Rauschen zu unterdrücken und die Verschiebung des Absorptionsmaximums als starken Effekt herauszustellen. Da oberhalb von $n=80$ keine eindeutig separierten Massenpeaks einzelner Clustergrößen vorlagen, wurden Bereiche in den Massenspektren ausgewertet, die mehrere Clustergrößen umfassten. Die Eichfaktoren für die Umwandlung der Flugzeit in die Massendomäne stammen aus der Auswertung des aufgelösten Bereichs mit $n \leq 80$. Die IR-Spektren zeigen das von Buch et al. 44 prognostizierte Absorptionsverhalten, jedoch zu größeren Clustergrößen hin verschoben. Zwischen $n=85 \pm 7$ und $n=425 \pm 25$ ist das Spektrum jenem amorpher Cluster mit der maximalen Intensität um $3350 \mathrm{~cm}^{-1}$ zuzuordnen. Bei $n=475 \pm 25$ (in rot gekennzeichnet) verschiebt sich das Maximum der Absorption zu kleineren Wellenzahlen, was den Übergang 


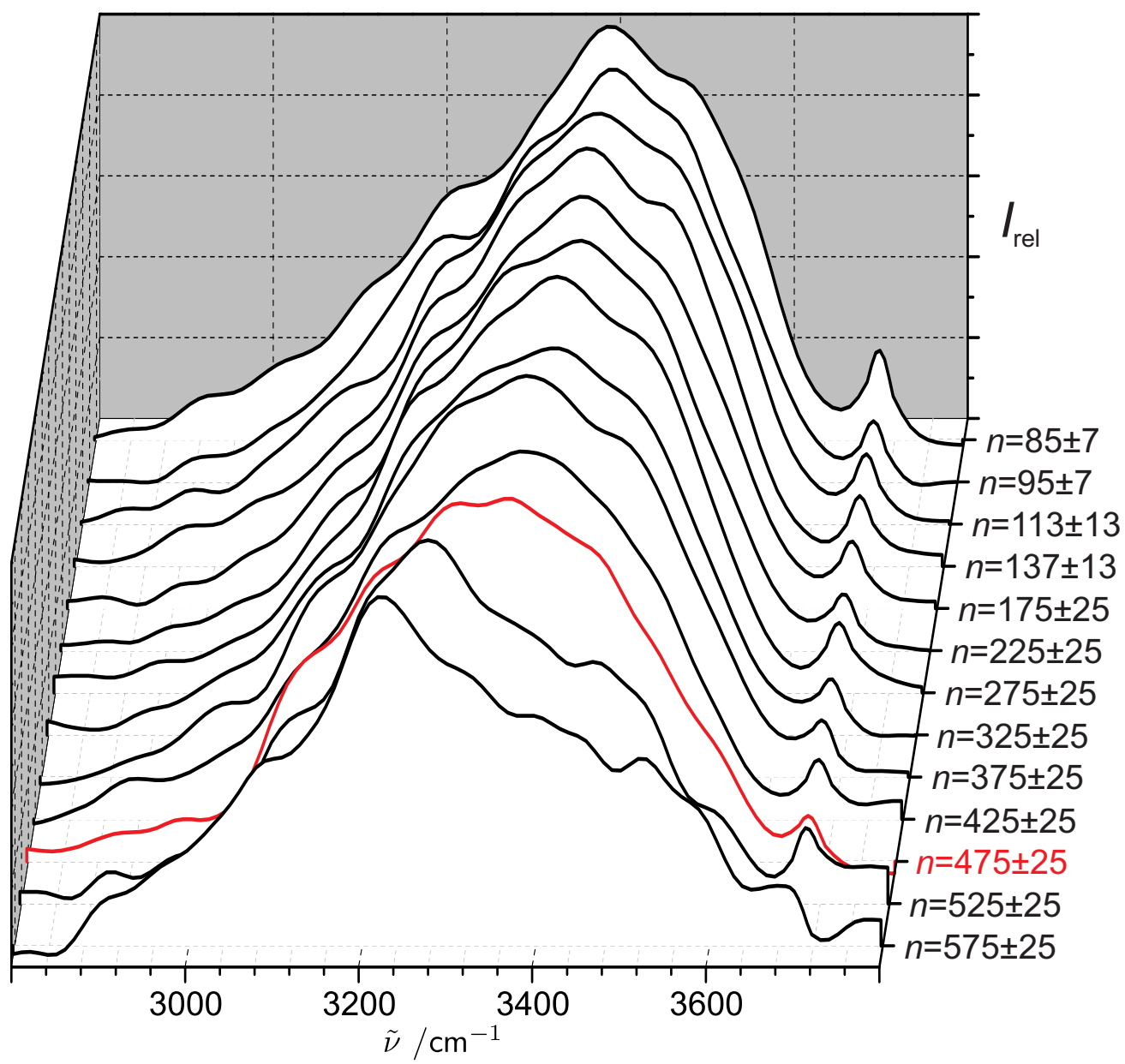

Abbildung 5.4.: Entwicklung der größenselektiv gemessenen IR-Spektren von $\mathrm{Na}\left(\mathrm{H}_{2} \mathrm{O}\right)_{n}$ von $n=85 \pm 7$ bis $575 \pm 25$. Die Expansionsbedingungen A sind $80 \%$ Wasser in Helium bei einem Stagnationsdruck von $4,5 \pm 0,1$ bar.

zu kristallinen Clustern andeutet. Diese Verschiebung setzt sich mit zunehmender Größe fort, sodass das Maximum bei $n=525 \pm 25$ bei $3220 \mathrm{~cm}^{-1}$ und bei $n=575 \pm 25$ bei $3200 \mathrm{~cm}^{-1}$ liegt. Für beide Clustergrößen kann deshalb davon ausgegangen werden, dass sie im Inneren zu einem großen Teil kristallin sind.

In Abbildung 5.5 ist die größenselektive Entwicklung der IR-Spektren von Wasserclustern mit $85 \pm 7$ bis $475 \pm 25$ Konstituenten bei einer Expansion mit Helium bei 3,9 $\pm 0,1$ bar Stagnationsdruck dargestellt (Expansionsfall B). Die weiteren Bedingungen sind eine Düsentemperatur von $\vartheta_{\text {Düse }}=135^{\circ} \mathrm{C}$ und eine Ofentemperatur von $\vartheta_{\text {Ofen }}=130^{\circ} \mathrm{C}$, was einem Anteil von $40 \%$ Wasser entspricht. Als Ionisations- 


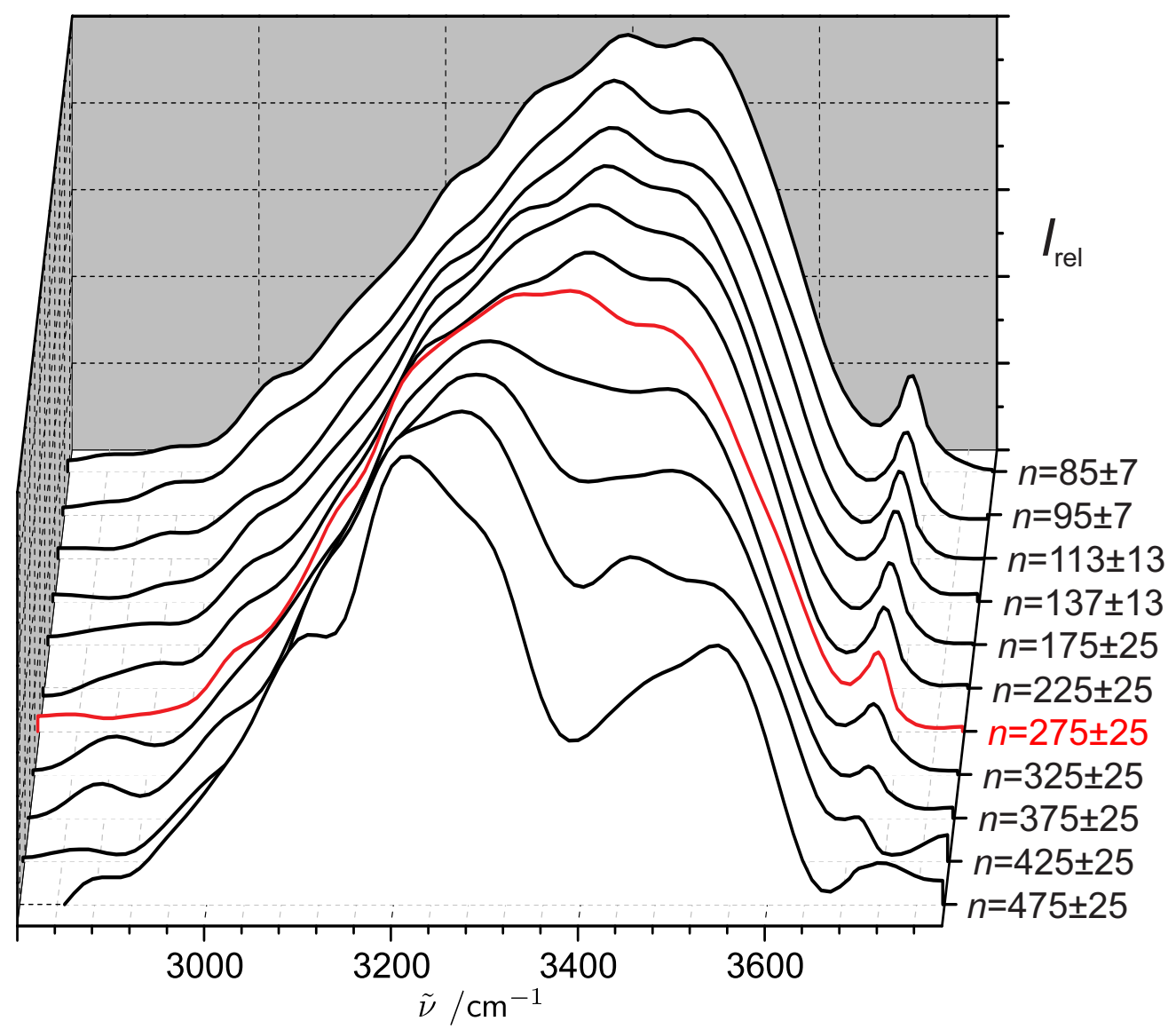

Abbildung 5.5.: Entwicklung der größenselektiv gemessenen IR-Spektren von $\mathrm{Na}\left(\mathrm{H}_{2} \mathrm{O}\right)_{n}$ von $n=85 \pm 7$ bis $475 \pm 25$. Die Expansionsbedingungen $\mathrm{B}$ sind $68 \%$ Wasser in Helium bei einem Stagnationsdruck von $3,9 \pm 0,1$ bar 47$]$.

wellenlänge wurde ebenfalls $390 \mathrm{~nm}$ verwendet (vgl. Tabelle A.2 im Anhang). Die Verminderung der Temperatur und des Stagnationsdrucks führt nicht nur dazu, dass die Größenverteilung zu kleineren Clustergrößen hin verschoben wird (vgl. Abb. 5.3), sondern wahrscheinlich auch zur Erhöhung der Clustertemperatur (vgl. Tabelle 5.1 und (11]). Zwischen $n=85 \pm 7$ und $n=225 \pm 25$ lässt sich ein zu amorphen Clustern passendes Absorptionsverhalten mit einem Maximum bei ungefähr $3400 \mathrm{~cm}^{-1}$ beobachten. Bei $n=275 \pm 25$, in rot gekennzeichnet, findet eine erste Verschiebung des Maximums, zu erkennen als Doppelpeakmuster um $3300 \mathrm{~cm}^{-1}$, statt. Dies deutet auf einen Übergang zwischen amorphen und kristallinen Clustern hin. Mit steigender Clustergrößen bleibt das Maximum bei diesem Wert, 
während sich um $3400 \mathrm{~cm}^{-1}$ ein lokales Minimum der Absorption ausbildet. Diese steigt bis $3500 \mathrm{~cm}^{-1}$ wieder an. Eine weitere Rotverschiebung des Absorptionsmaximums bis auf $3200 \mathrm{~cm}^{-1}$ ist zwischen $n=375 \pm 25$ und $n=475 \pm 25$ zu beobachten. In diesem Clustergrößenbereich ist davon auszugehen, dass das Clusterinnere zum größten Teil kristallin ist.

Die nochmalige Verringerung der Temperatur des Ofens um $10 \mathrm{~K}$ und des Stagnationsdrucks um 0,5 bar führt zur Bildung, wenn man den geschätzten Trend fortsetzt, von etwa $20 \mathrm{~K}$ wärmeren Clustern. Die größenselektive Entwicklung der IR-Spektren aus einer Verteilung mit den oben beschriebenen Expansionsbedingungen $\left(\vartheta_{\text {Düse }}=130{ }^{\circ} \mathrm{C}\right.$ und $\left.\vartheta_{\text {Ofen }}=120^{\circ} \mathrm{C}\right)$ ist in Abbildung 5.6 dargestellt. Die

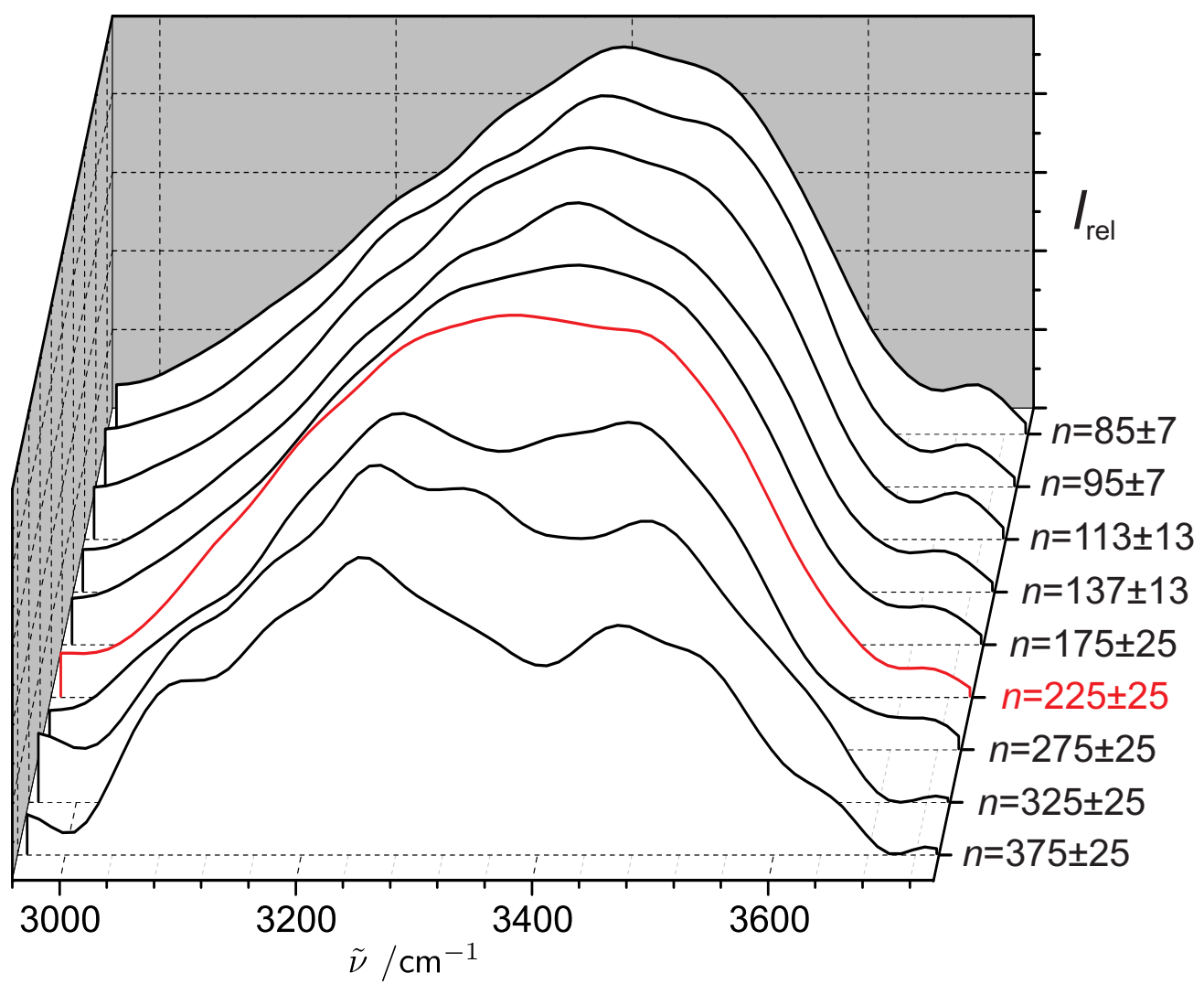

Abbildung 5.6.: Entwicklung der größenselektiv gemessenen IR-Spektren von $\mathrm{Na}\left(\mathrm{H}_{2} \mathrm{O}\right)_{n}$ von $n=85 \pm 7$ bis $375 \pm 25$. Die Expansionsbedingungen $\mathrm{C}$ sind $57 \%$ Wasser in Helium bei einem Stagnationsdruck von 3,5 bar.

leichte Blauverschiebung des Absorptionsmaximums und die, im Vergleich zu den 
vorherigen Messreihen, breitere Absorption der amorphen Clustergrößen $n=85 \pm 7$ bis $n=175 \pm 25$ sind konsistent mit der höher geschätzten Clustertemperatur. Die erste Verschiebung der maximalen Absorption und damit das Einsetzen der Kristallisation findet bei der Clustergröße $n=225 \pm 25$ statt. Ab $\left(\mathrm{H}_{2} \mathrm{O}\right)_{275 \pm 25}$ bleibt das Maximum bei einem Wert von $3220 \mathrm{~cm}^{-1}$. Es bildet sich ein Intensitätseinbruch bei $3350 \mathrm{~cm}^{-1}$ aus, der im weiteren Verlauf wie im vorherigen Fall zu $3400 \mathrm{~cm}^{-1}$ wandert.

Bisher zeigen die Spektren ein von der geschätzten Endtemperatur der Cluster abhängiges Einsetzen der Kristallisation. Je kälter die Temperatur, desto größer ist der Cluster, bei dem die Ausbildung der kristallinen Struktur einsetzt. Allerdings werden die Cluster vermutlich in der Düse bei höheren Temperaturen kristallisieren und diese Temperaturen scheinen mit der Endtemperatur zu korrelieren. Um den Einfluss der Expansionsbedingungen auf den Kristallistionsprozess weiter zu testen, bietet sich ein Wechsel des Trägergases und eine weitere Reduktion des Stagnationsdruckes an. Im Folgenden soll daher die größenselektive Untersuchung der Kristallisation bei einer Expansion mit Neon als Trägergas vorgestellt werden.

\subsubsection{Kristallisation bei Neon als Trägergas}

Die Entwicklung der IR-Spektren im Bereich zwischen $n=85 \pm 7$ und $375 \pm 25$ ist in Abbildung 5.7 dargestellt. Es fand für die Erzeugung der Spektren analog zu den Helium-Expansionsbedingungen eine Mittelung aus zwei Messtagen, dem 16. und 21. August 2012, sowie eine zehnfache Glättung statt. Die detaillierten Expansionsbedingungen können der Tabelle A.2 im Anhang entnommen werden. Die Ionisation wurde, anders als bei den vorherigen Spektren, bei $388 \mathrm{~nm}$ durchgeführt. Die Expansion mit Neon ermöglicht trotz geringerem Signals in den Massenspektren eine Auswertung bis zur letzten möglichen Größenscheibe (vgl. Abbildung 5.3). Das Spektrum von $n=85 \pm 7$ ähnelt jenem der kalten Expansion mit Helium (Expansion A). Das Einsetzen der Kristallisation scheint bei $n=225 \pm 25$ vorzuliegen. Dies wird im Gegensatz zu den vorherigen Fällen nicht durch eine Verschiebung der maximalen Absorption zu kleineren Wellenzahlen deutlich, sondern durch die Verbreiterung des Signals, sowie durch die Ausbildung eines lokalen Minimums bei $3400 \mathrm{~cm}^{-1}$, was auch in den Größenscheiben $325 \pm 25,375 \pm 25$ und $425 \pm 25$ in Abbildung 5.5 für die Expansion B, bzw. auch in den Größenscheiben 325 \pm 25 , 


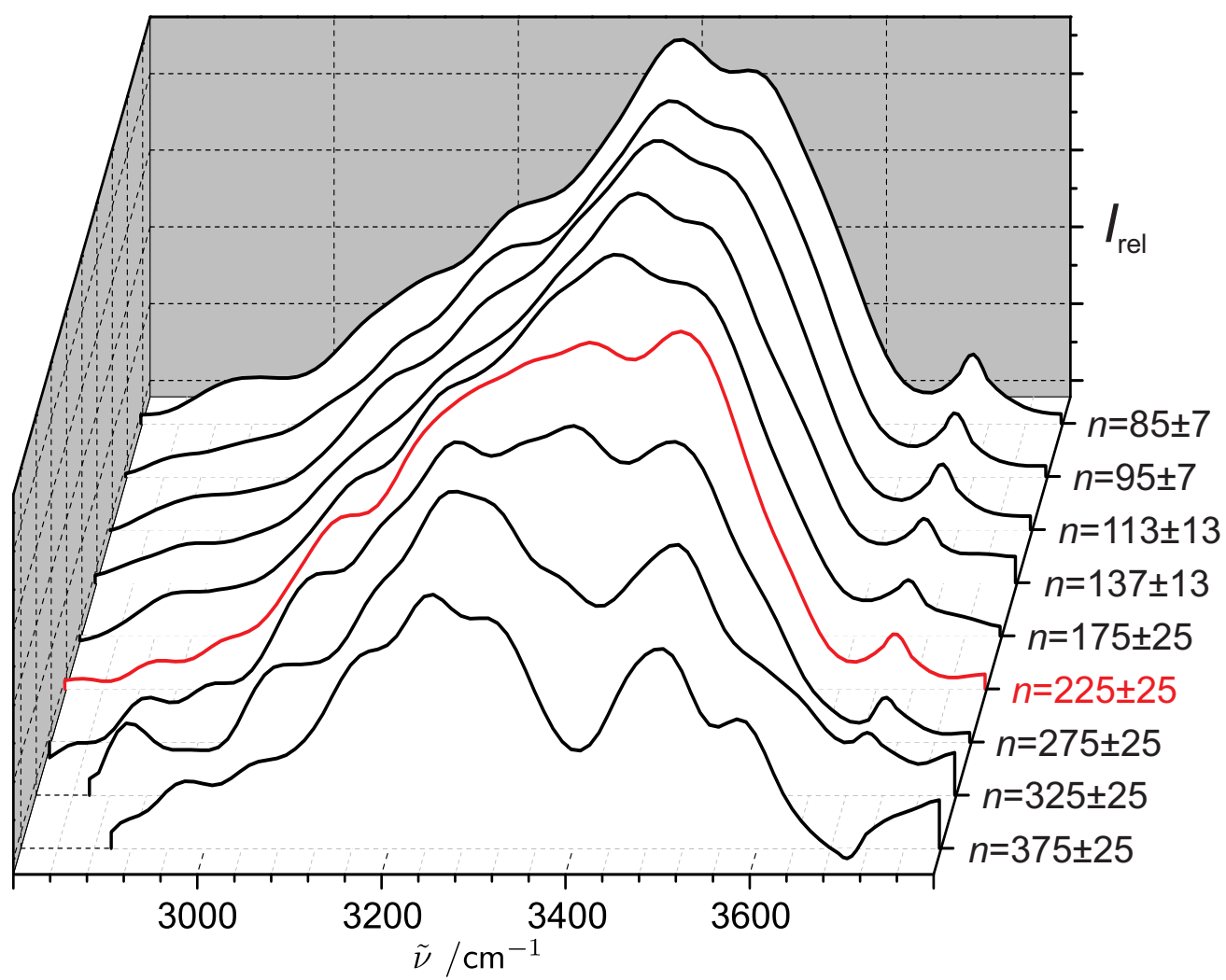

Abbildung 5.7.: Entwicklung der größenselektiv gemessenen IR-Spektren von $\mathrm{Na}\left(\mathrm{H}_{2} \mathrm{O}\right)_{n}$ von $n=85 \pm 7$ bis $375 \pm 25$. Die Expansionsbedingungen sind $43 \%$ Wasser in Neon bei einem Stagnationsdruck von $2,6 \pm 0,3$ bar.

$375 \pm 25$ in Abbildung 5.6 für die Expansion C zu erkennen ist. Im weiteren Verlauf der Clustergrößen ist der Peak des kristallinen Kerns um $3200 \mathrm{~cm}^{-1}$ ausgebildet und stellt für $n=325 \pm 25$ und $n=375 \pm 25$ das Absorptionsmaximum dar. In Clustern dieser Größe scheint die Ausbildung des kristallinen Kerns abgeschlossen zu sein. 
5.2. Experimentelle Spektren

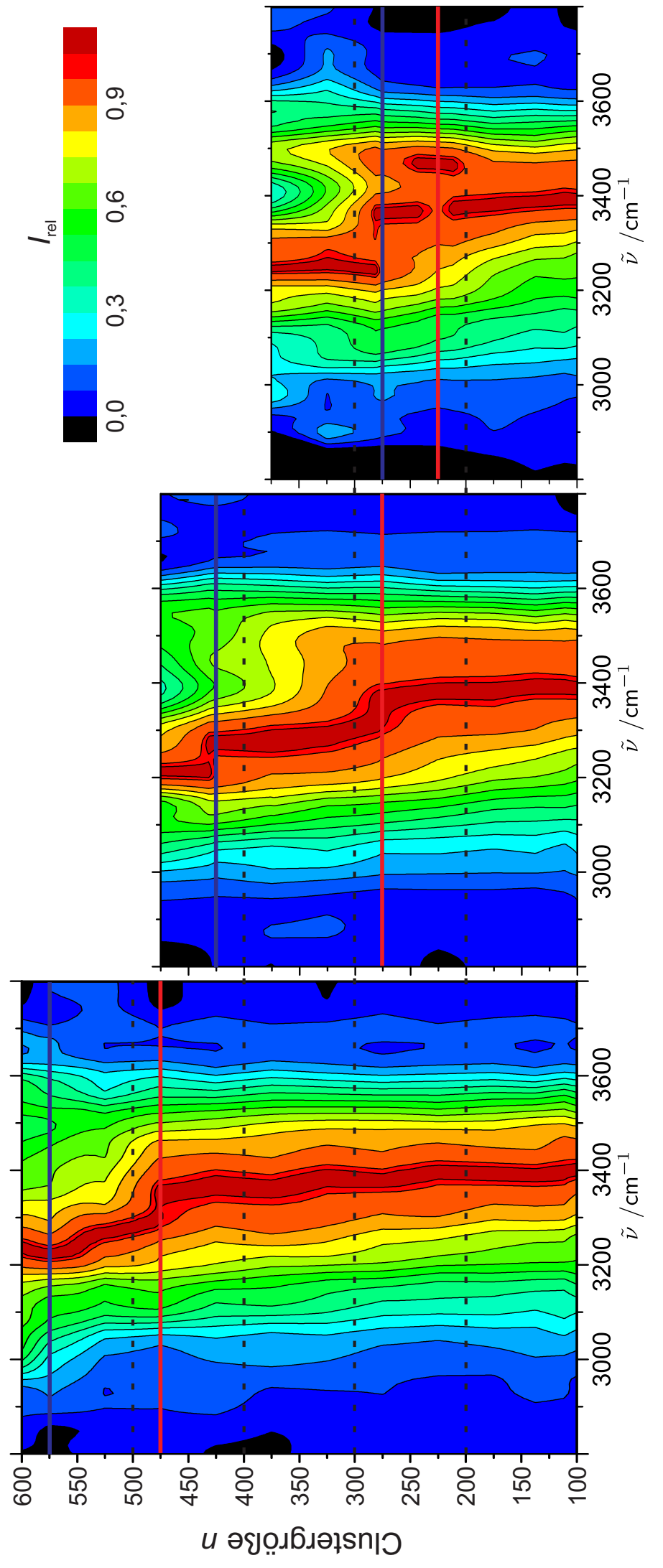

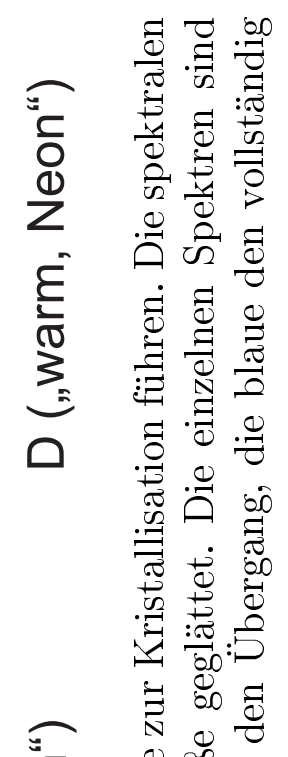

हᄐ :

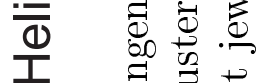

है

릉

¿ $\quad$ है

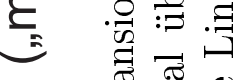

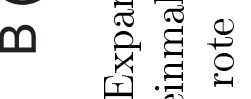

ธิ

- 듕

$\forall \stackrel{g}{0}$

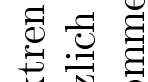

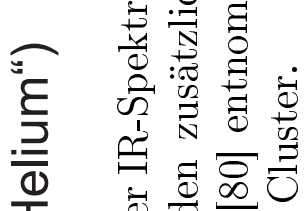

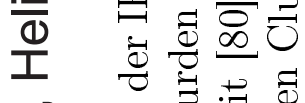

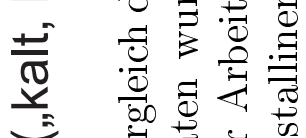

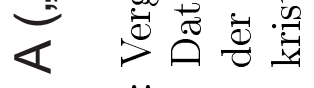

\begin{tabular}{c}
$\ddot{0}$ \\
10 \\
0 \\
0 \\
$\Xi$ \\
$\vdots$ \\
\hdashline$\frac{0}{7}$ \\
$\frac{1}{2}$
\end{tabular} 


\subsubsection{Vergleich der unterschiedlichen Expansionen}

Zusammenfassend lässt sich in allen hier gezeigten Beispielen der größenselektiv gemessenen Kristallisation von Wasserclustern ein sehr ähnliches Verhalten erkennen. Nach einem Größenbereich mit sehr ähnlichen Spektren setzt mehr oder weniger prompt eine Veränderung der Absorption mit Rotverschiebung des Absorptionsmaximums ein. Dieser Befund soll im Folgenden mit Hilfe einer anderen Form der Visualisierung betrachtet werden.

Der Vergleich der größenselektiven Entwicklung der IR-Spektren dreier unterschiedlicher Expansionsbedingungen wird in Abbildung 5.8 vollzogen. Auf die Expansion C (,warm, He“) wurde aufgrund der Ähnlichkeit zum Neon-Fall und des schlechteren Signal-zu-Rausch-Verhältnisses verzichtet. Es erfolgte für die spektralen Daten im Gegensatz zu den vorherigen Darstellungen noch eine Glättung über die Clustergröße. Die Intensität der IR-Spektren jeder Größenscheibe wurde durch die maximale Absorption normiert. Alle Spektren weisen bei kleinen Clustergrößen ein amorphes Verhalten auf. Der Übergang zum kristallinen Cluster stellt eine Änderung der Absorption mit einer Verschiebung des Maximums zu kleineren Wellenzahlen dar. Dieser wird durch die rote Linie gekennzeichnet und findet im kalten Expansionsfall A bei $n=475 \pm 25$, in Expansion B (,medium“) bei $n=275 \pm 25$ und im wärmsten Fall $\mathrm{D}$ bei $n=225 \pm 25$ statt. Bei letzterem tritt die Änderung des Absorptionsverhaltens mit der Ausbildung eines Maximums um $3500 \mathrm{~cm}^{-1}$ auf. Mit zunehmender Clustergröße tritt eine weitere Verschiebung der Absorption auf, gekennzeichnet durch die blaue Linie. Während dies in der Expansion B und D abrupt auftritt $(n=425 \pm 25$ bzw. $n=275 \pm 25)$, ist die Verschiebung im kalten Expansionsfall A kontinuierlich und endet bei der letzten Größenscheibe mit $n=575 \pm 25$.

Bisher erfolgte die Darstellung der Kristallisation mit Hilfe von mehrfach geglätteten Spektren. Bei dieser Datenbehandlung gehen aber spektrale Informationen verloren und nur grobe Muster bleiben erkennbar. In Abbildung 5.9 sind daher die Rohdaten der amorphen und kristallinen Cluster aus den Expansionen A („kalt") in blau, B („,medium “) in grün, D (,warm, Neon“) in rot, sowie die Mittelung der drei Spektren gegenübergestellt. Als Vertreter der amorphen Strukturen wurde die Größenscheibe $n=113 \pm 13$ ausgewählt. Die Spektren stellen jeweils eine Mittelung aus den Rohdaten von drei Messreihen dar. Lediglich der Fall „kalt“ besteht nur 


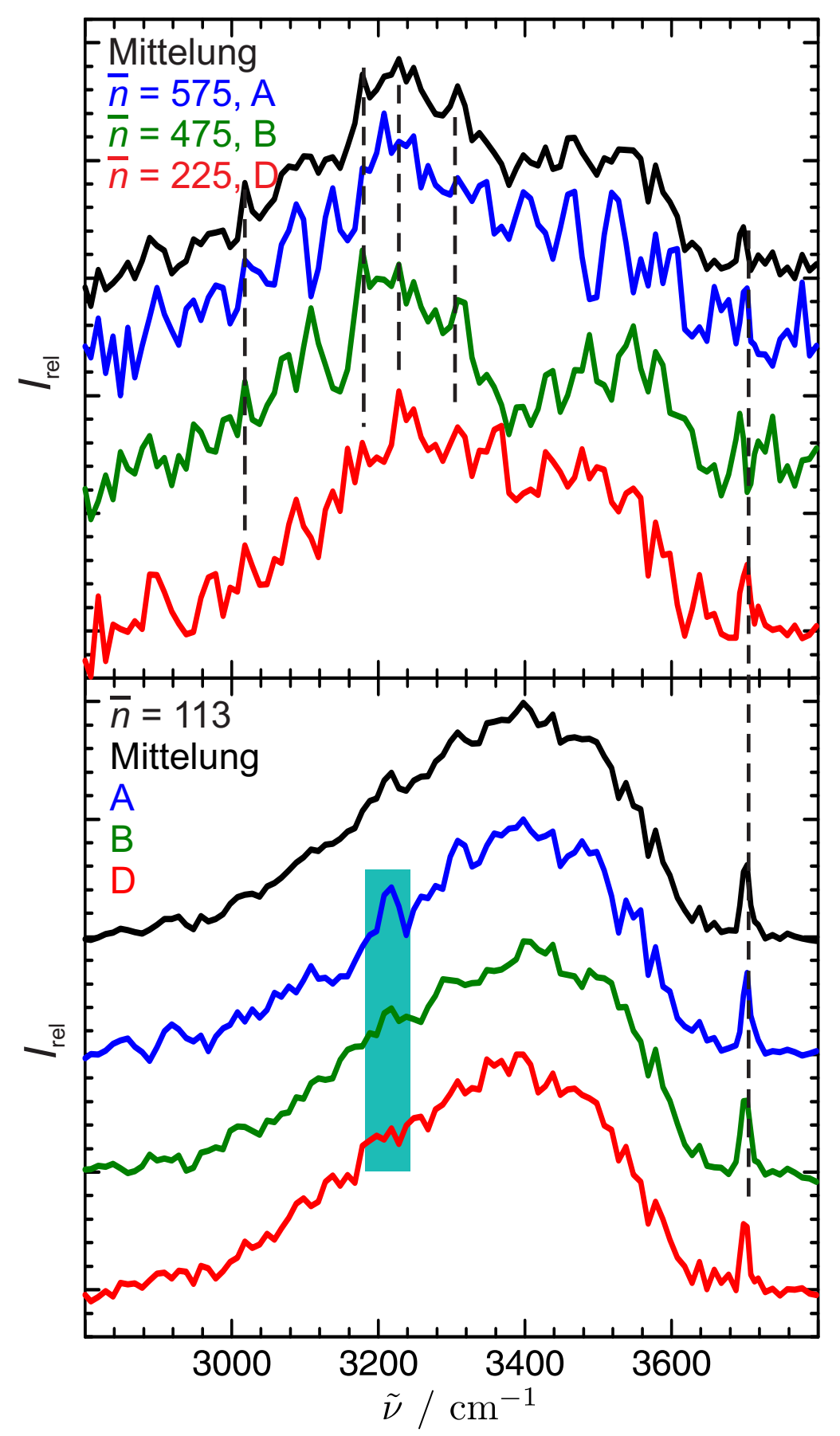

Abbildung 5.9.: Vergleich der IR-Spektren von amorphen und kristallinen Strukturen der Expansionsbedingungen A (,,kalt") in blau, B (,medi$u^{\prime \prime}$ ) in grün und D (,warm, Neon") in rot, sowie eine Mittelung der drei Spektren in schwarz. Weitere Erläuerungen im Text. 
aus den oben genannten zwei Messreihen. Die Spektren sind in guter Übereinstimmung zueinander. Sie zeigen eine breite Absorption zwischen $3000 \mathrm{~cm}^{-1}$ und $3600 \mathrm{~cm}^{-1}$ mit einem Absorptionsmaximum bei $3400 \mathrm{~cm}^{-1}$, lediglich die freie $\mathrm{OH}-$ Streckschwingung um $3700 \mathrm{~cm}^{-1}$ ist separiert. Breite Peaks treten bei $3300 \mathrm{~cm}^{-1}$ und $3200 \mathrm{~cm}^{-1}$ auf. Letzterer zeigt eine starke Temperaturabhängigkeit und ist vielleicht auf eine Fermi-Resonanz der Streckschwingung mit dem Oberton der Biegeschwingung 15 der in den amorphen Clustern auftretenden Fünfringe zurückzuführen.

Die Spektren der kristallinen Strukturen in den Fällen A (,kalt“) und B (,medium“) entsprechen den gemittelten Rohdaten der letzten auswertbaren Größenscheibe. Für die warmen Expansionsbedingungen wurden die Spektren über den Größenbereich $n=175 \pm 25$ bis $n=275 \pm 25$ für drei Messreihen gemittelt. Das Absorptionsverhalten unterscheidet sich stark von jenem der amorphen Cluster und zeigt spezifische scharfe Peakmuster um $2900 \mathrm{~cm}^{-1}$ und $3000 \mathrm{~cm}^{-1}$, dem Bereich der Oszillation der dreifach koordinierten DAA-Moleküle. Im Bereich der Oszillatoren des kristallinen Kerns zwischen $3200 \mathrm{~cm}^{-1}$ und $3300 \mathrm{~cm}^{-1}$ weist die Absorption der drei Spektren insgesamt einige identische Wendepunkte auf. Im weiteren Verlauf des Spektrums, dem Bereich der DDA-Oszillatoren und der Absorption amorpher Cluster, treten die meisten Unterschiede auf. Deutlich wird dies schon in den Konturauftragungen in Abbildung 5.8. Aufgrund der unterschiedlichen Oberflächen der Cluster mit kristallinem Kern weist Fall D („warm, Neon“) eine höhere Intensität um $3400 \mathrm{~cm}^{-1}$ auf. Die Spektren der Expansionsbedingungen A und B zeigen hingegen um $3500 \mathrm{~cm}^{-1}$ ein Doppelpeakmuster, welches aufgrund der unterschiedlichen Temperaturen und Größen nicht ganz übereinstimmt. Die freie $\mathrm{OH}-$ Streckschwingung lässt sich nur schwer identifizieren, lediglich für $\bar{n}=225$ ist sie eindeutig separiert. Bei $\bar{n}=475$ ist sie im Vergleich zum Spektrum von $\bar{n}=225$ um $5 \mathrm{~cm}^{-1}$ verschoben. Für $\bar{n}=575$ zeigt sie die schwächste Intensität.

Die Mittelung aller amorphen und kristallinen Spektren zeigt, dass die in Einzelspektren erkennbaren Absorptionsmuster der kristallinen Cluster bestehen bleiben, während die Absorption des amorphen Clusters deutlich glatter wird. 


\subsection{Diskussion}

Die hier vorgestellten, über Wirkungsspektroskopie aufgenommenen IR-Spektren liefern ein vollständig größenselektives Bild der Kristallisation von Wasserclustern und sind in guter Übereinstimmung mit dem von Buch et al. [44 prognostizierten Verhalten. Das Einsetzen der Kristallisation sowie die Ausbildung des kristallinen Kerns sind dabei von den Expansionsbedingungen und damit vom Temperaturverlauf des Abkühlungsprozesses der Cluster abhängig. Die Kristallisation beginnt bei $n=225 \pm 25$ für die als warm abgeschätzten und bei $n=475 \pm 25$ für die als am kältesten abgeschätzten Expansionsbedingungen und liegt somit in allen Fällen in dem von Torchet und Mitarbeitern eingegrenzten Bereich von $n=200-1000$ 45. Das frühe Einsetzen der Kristallisation für warme Expansionsbedingungen wurde bereits durch die Messungen von Fujii und Mitarbeitern [25,27] gezeigt. Im Abbildung 5.10 sind die größenselektiven IR-Spektren von kationischen Wasserclustern der Form $\mathrm{H}^{+}\left(\mathrm{H}_{2} \mathrm{O}\right)_{n}$ abgebildet. Im Gegensatz zur IR-CIPI Methode, die in einer Messung den gesamten Größenbereich abdeckt, wird hier die zu untersuchende Größe vorher ausgewählt und mittels IRMPD dissoziiert. Die Temperatur der Cluster wird auf $150 \mathrm{~K}$ bis $200 \mathrm{~K}$ abgeschätzt und liegt somit nah am evaporativen Ensemble 163. Aufgetragen sind die IRMPD Spektren von $\mathrm{H}^{+}\left(\mathrm{H}_{2} \mathrm{O}\right)_{n}$ in dem Bereich zwischen $n=100$ bis $n=221$ im Vergleich mit den Spektren von flüssigem Wasser bei $298 \mathrm{~K}$ in rot, unterkühltem Wasser bei $240 \mathrm{~K}$ in orange, hexagonalem Eis bei $235 \mathrm{~K}$ in blau und dem natriumdotierten Cluster der Größe $n=113 \pm 13$ aus dem Expansionsfall B (Tabelle 5.1 auf 92) in grün. Die größenselektiven Spektren zeigen, wie flüssiges und unterkühltes Wasser, eine breite Absorption, das Maximum von $n=100$ liegt dabei bei $3350 \mathrm{~cm}^{-1}$, die freie OH-Streckschwingung ist bei allen klar separiert. Die Spektren der kationischen Cluster der Größe 100 und 150 sind in Übereinstimmung mit dem der amorphen, natriumdotierten Cluster. Jedoch scheint bei $n=150$ die Kristallisation zu beginnen, da das Maximum der Absorption zu $3300 \mathrm{~cm}^{-1}$ wandert. Mit zunehmender Clustergröße bleibt das Maximum bei diesem Wert und ein signifikantes Ansteigen der Absorption bei $3220 \mathrm{~cm}^{-1}$ kann nicht beobachtet werden.

Eine offene Frage, die sich aus der Betrachtung der experimentellen Spektren ergibt, ist, ob sich die Spektren der kristallinen Cluster allein durch das Auftreten eisähnlicher Isomere erklären lassen. Schließlich gibt es Unterschiede (wie schon 


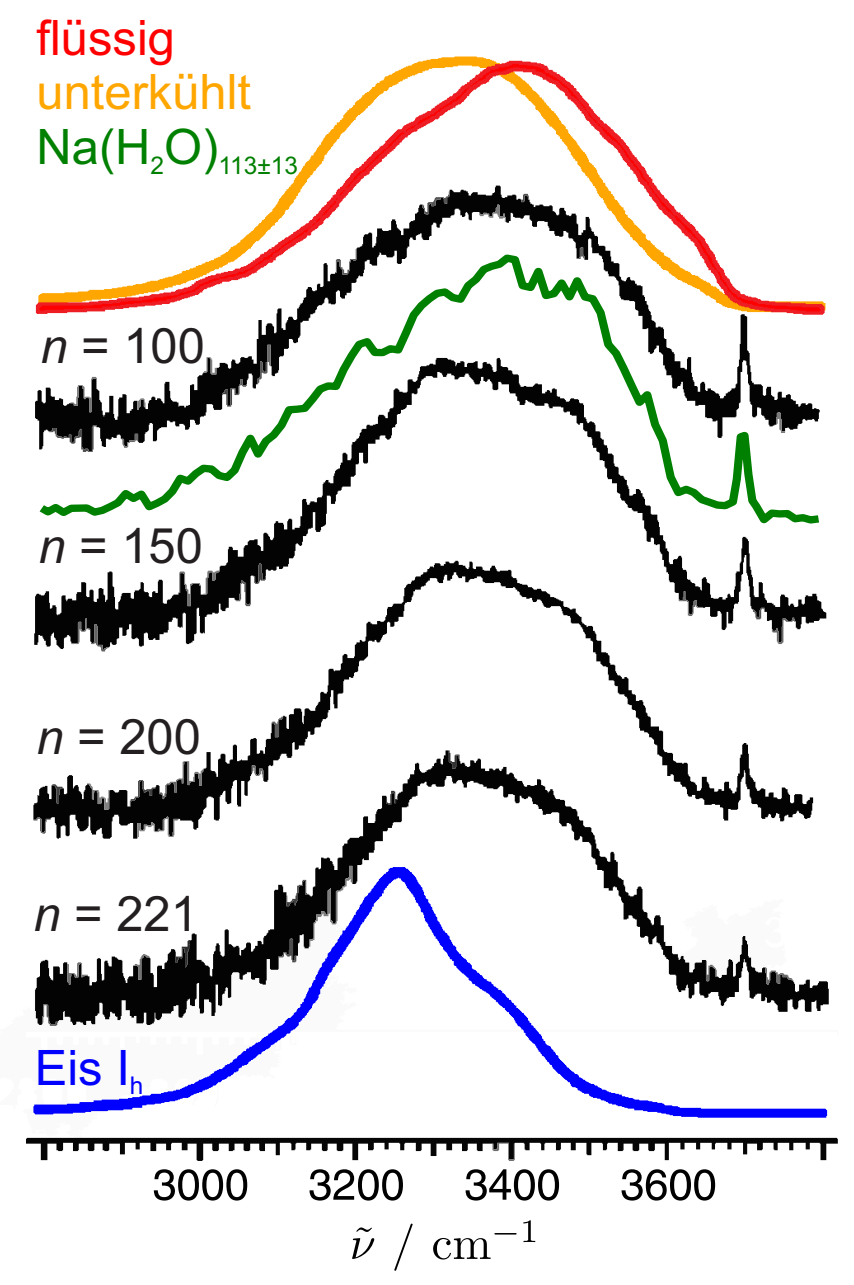

Abbildung 5.10.: Größenselektive Entwicklung der IR-Spektren von $\mathrm{H}^{+}\left(\mathrm{H}_{2} \mathrm{O}\right)_{n}$ im Vergleich zu den Spektren von flüssigem Wasser bei $298 \mathrm{~K}$ (rot), unterkühltem Wasser bei $240 \mathrm{~K}$ (orange), hexagonalem Eis bei $235 \mathrm{~K}$ (blau) und dem natriumdotierten Cluster der Größe $n=113 \pm 13$ aus dem Expansionsfall B (grün). Die Graphik ist verändert nach [25,27]. Die Daten für flüssiges und unterkühltes Wasser sowie Eis $\mathrm{I}_{\mathrm{h}}$ stammen aus den Publikationen 161,162.

in Kapitel 4.3 .2 gezeigt) zwischen den hier vorliegenden experimentellen und den von Buch et al. simulierten Spektren [44], die möglicherweise auf die verwendete Wirkungsspektroskopie-Methode zurückzuführen sind. Eine weitere mögliche Erklärung wäre die von Berry et al. für Cluster postulierte Koexistenz zweier Phasen, die in einem bestimmten Temperaturbereich auftreten sollte [164. Auch Bullock et al. [87 fanden in MD-Simulationen der Abkühlung und Kristallisation einer 10\% 
ionischen, wässrigen Lösung, dass in einem kristallinen $10 \mathrm{~nm}$ großen Nanopartikel $45 \%$ des Wasserclusters amorph bleiben. Der Rest stellt eine Mischung aus den Eisphasen $I_{c}$ und $I_{h}$ dar. Betrachtet man das IR-Spektrum für einen Bereich, in dem nur noch wenig Massensignal vorhanden ist, wie es in Abbildung 5.11 für die Clustergröße $\bar{n}=475$ für die Expansionsbedingungen C (,warm, Helium“) geschehen ist, so entsteht ein Entvölkerungsspektrum, dessen Signatur in Übereinstimmung mit der Absorption der amorphen Clustergröße $\bar{n}=113$ ist. Diese Abbildung zeigt die ungeglätteten und ungemittelten IR-Rohdaten vom 08.03.2012 in rot. Die gepunkteten Linien repräsentieren den Wert ohne Signalab- oder zunahme. Jedoch tritt die IR-induzierte Signalabnahme nicht in allen Expansionsfällen auf, lediglich im hier gezeigten Beispiel, sowie in den Spektren vom 27.03.12 ab einer Clustergröße von $n=575 \pm 25$ (Expansionsbedingungen B) und vom 13.04.12 für die letzte Größenscheibe $\bar{n}=950$ (Expansionsbedingungen A) ist dies bei einer Ionisationswellenlänge von $390 \mathrm{~nm}$ zu beobachten. Erfolgt die Ionisation mit $360 \mathrm{~nm}$, erhält man ein von den Expansionsbedingungen abhängiges Abnahmesignal, das ungefähr bei der gleichen Clustergröße einsetzt wie die Kristallisation. Dieses tritt für die Expansion C ab $n=275 \pm 25$ und für die Expansion B ab $n=375 \pm 25$ auf und nimmt mit steigender Clustergröße in seiner Intensität zu. Das schwarze Spektrum aus Abbildung 5.11 stellt eine Mittelung der auf maximales negatives Signal skalierten IR-wellenlängenabhängigen Signalabnahme von 24 Spektren unterschiedlicher Größenbereiche dar. Trotz Unterschieden, die vermutlich aufgrund positiver Beiträge durch kristalline Anteile zurückzuführen sind, ähneln sich die gemittelten Absorptionen des Abnahmesignals und der amorphen Clustergröße $\bar{n}=113$ (in schwarz dargestellt).

Die experimentellen Ergebnisse lassen folgenden Schluss für die Kristallisation von Wasserclustern zu: Cluster mit 100 Molekülen sind unter allen experimentellen Bedingungen weitestgehend amorph. Das Einsetzen der Kristallisation ist sowohl stark von der Clustergröße als auch den Expansionsbedingungen abhängig, wie durch Vergleich der Tabelle 5.1 und Abbildung 5.9 ersichtlich wird. Ist die Abkühlrate hoch und damit die Endtemperatur niedrig, wird bei Clustern mit bis zu 450 Molekülen der amorphe Zustand ausgefroren. Dies ist ein analoger Prozess zur Bildung von LDA durch massive Abkühlung mit einer Abkühlrate von $10^{7}$ Kelvin pro Sekunde 85 . Durch das Einsetzen der Kristallisation wird die Kristallisationsenthalpie freigesetzt, die zu einer Erwärmung des Clusters führt. 


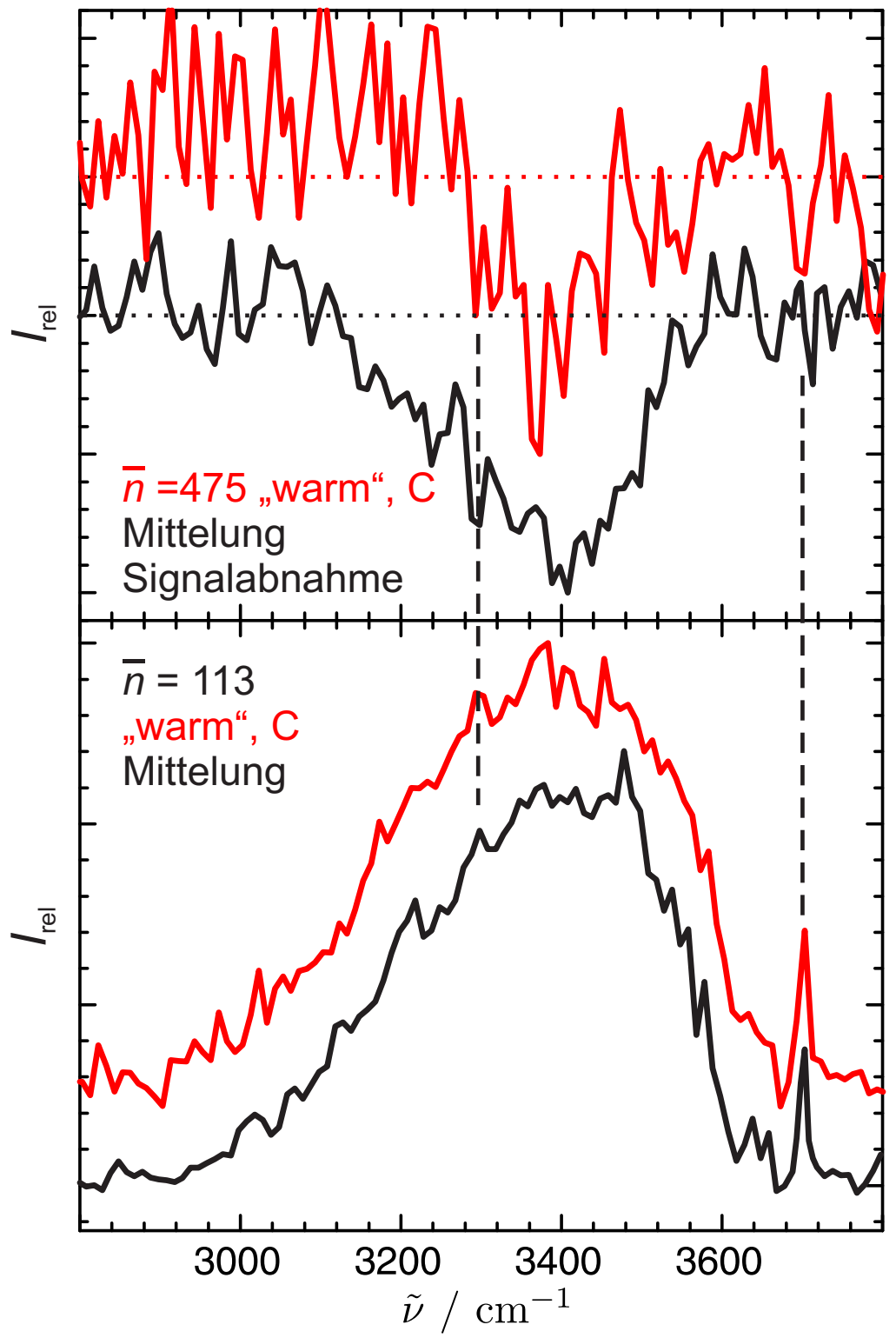

Abbildung 5.11.: Vergleich der IR-induzierten Signalabnahme mit dem Spektrum der amorphen Größenscheibe $\bar{n}=113$. In rot: die unbehandelten IR-Spektren der $\left(\mathrm{H}_{2} \mathrm{O}\right)_{n}$-Cluster für $\bar{n}=475$ und $\bar{n}=113$ vom 08.03.2012 (Expansionsbedingungen $\mathrm{C}$ ). Oben in schwarz: Mittelung der IR-wellenlängenabhängigen Signalabnahme vom 27.03.12 (B), 13.04.12 (A), 02.05.12, 04.05.12 (beide C, $\lambda_{\mathrm{PI}}=$ $360 \mathrm{~nm})$ und $09.05 .12\left(\mathrm{~B}, \lambda_{\mathrm{PI}}=360 \mathrm{~nm}\right)$. Unten in schwarz: Mittelung der IR-Spektren der amorphen Größenscheibe $\bar{n}=113$ vom 27.03.2012, 13.04.12, 02.05.12, 04.05.12 und 09.05.12. Weitere Erläuterungen im Text. 
Dieser Energiebeitrag könnte bei zeitverzögert einsetzender Kristallisation - da er in der adiabatischen Überschallexpansion im Aggregat bleibt und nicht an die Umgebung abgeführt werden kann - zu einer Verflüssigung des kristallinen Clusters führen [164. Ein Unterschied hierzu liegt bei der Untersuchung von Eispartikeln in Hüllstromzellen vor 43, 44. In diesem Experiment kann das Aggregat die freiwerdende Enthalpie an die Umgebung abgeben, sodass das Ausfrieren hier möglich ist (vgl. Abbildung 4.6 auf Seite 81). Ist die Endtemperatur des Molekularstrahls höher, so ergibt sich eine längere Verweilzeit im für die Kristallisation günstigen unterkühlt flüssigen Zustand, so dass auch kleinere Cluster mit vermutlich höheren Barrieren kristallisieren können. Zudem tritt ein weiterer Effekt auf, der zum Intensitätseinbruch in Expansion B („medium“) und D (,warm, Neon“) um $3400 \mathrm{~cm}^{-1}$, sowie zur vollständigen Entvölkerung bei $\bar{n}=475$ für den Fall $\mathrm{C}$ (,warm, Helium“) führt: Erhöht man die Ionisationsenergie, was zu einer Stärkung der Signalabnahme und einer Schwächung der Signalzunahme führt [41], tritt diese Signalabnahme im kristallinen Größenbereich ebenfalls in dem Expansionsfall B auf. Das Absorptionsmuster entspricht dabei dem eines amorphen Clusters. Ob diese Verflüssigung über die kristalline Phase abläuft lässt sich nicht zweifelsfrei nachweisen, ein Übergang zweier amorpher Phasen wäre ebenfalls denkbar und wurde von der Gruppe von von Issendorf für kationische und anionische Wassercluster beobachtet 90,165 . Eine Unterscheidung amorpher Phasen ist durch IR-Spektroskopie schwer möglich, da, ähnlich wie bei flüssigem und unterkühltem Wasser (vgl. Abb. 5.10), lediglich eine leichte Verschiebung der maximalen Absorption stattfindet. Die freiwerdende Enthalpie in Verbindung mit der Absorption der IR-Photonen kann im Falle der rückverflüssigten Cluster zu einer evaporativen Kühlung und somit zur Dissoziation [163 führen, in Analogie zum IRMPD-Verfahren bei kationischen Clustern [25,27. 


\section{Strukturuntersuchung einzelner Clustergrößen}

Die bisherige Betrachtung der experimentell ermittelten IR-Spektren konzentrierte sich auf generelle Trends in einem Clustergrößenbereich von 80 bis 600 Wassermolekülen. Die große Anzahl an Oszillatoren führte zu einer breiten Absorption im IR-Spektrum. Bereits im Kapitel 2.2.1 erfolgte ein Überblick über die durch experimentelle oder quantenchemische Methoden postulierten Strukturen. Im Gegensatz zur Flüssigkeit, bei der die meisten Moleküle voll koordiniert sind, bilden kleine Cluster mit wenigen Wassermolekülen $(n<17)$ Strukturen aus, bei denen sich alle Moleküle auf der Oberfläche des Clusters befinden [17, 68, 103, 166, 169. Ab der Clustergröße $n=6$ zwingt die Maximierung der Koordination die Moleküle zur Ausbildung von dreidimensionalen Wasserstoffbrückenbindungsnetzwerken [17,68 70]. Das Auftreten und die Isomerie der Cluster mit 7 bis 10 Molekülen ist durch zahlreiche Untersuchungen detailliert beschrieben [9,24,103]. Jedoch ist keines der Moleküle in diesen Isomeren vierfach koordiniert. Aus vorherigen quantenchemischen Studien von Hartke und Lagutschenkov et al. wird die kritische Clustergröße, bei der zum ersten mal ein Wassermolekül im Inneren eines Clusters vier Wasserstoffbrückenbindungen ausbildet, mit $n=17$ benannt [73, 74. Der experimentelle Nachweis dieser Strukturen war bisher nicht möglich. Lediglich für ionische Wassercluster gibt es Studien, die experimentelle und auf Rechnungen basierende Daten kombinieren, um die Hydratation in diesen Systemen auf mikroskopischer Ebene zu charekterisieren 170,171.

In der Dissertation von Dr. Richard M. Forck wurden verschiedenen Strukturisomere für natriumdotierte Cluster der Größen $n=2$ bis 5 untersucht 41]. Das Natrium hat in diesem Größenbereich einen großen Einfluss auf die Ausbildung der Strukturen. Buck et al. publizierten jeweils 4 Strukturisomere für die Größen $n=8,10,16$ und 20 [39. Die Ionisation in diesem Experiment erfolgte mit $400 \mathrm{~nm}$ 
$(3,1 \mathrm{eV})$, was $\mathrm{zu}$ einem hohen Anteil an Isomeren mit teilsolvatisiertem Elektron geführt haben könnte (vgl. Abbildung 2.2), sodass der Fokus dieser Studie auf die Interaktion des Wasserclusters mit dem Na-Atom und die Bildung des solvatisierten Elektrons gelegt wurde. In Kapitel 4.1 wurde durch den Vergleich mit anderen Messverfahren gezeigt, dass durch geeignete Auswahl von Expansionsbedingungen und Ionisationswellenlänge die Störung durch das Natriumatom ab einer Clustergröße von ungefähr $n=19$ lediglich einen geringen Einfluss auf die Topologie des Wasserstoffbrückennetzwerks im Wassercluster hat.

\subsection{Clustergröße $n=20$}

Zunächst soll die Clustergröße $n=20$ betrachtet werden, da hierfür eine Vielzahl von publizierten Strukturfamilien mit den dazugehörigen Bindungsenergien und IR-Spektren vorliegen $73,74,172,173$. Einer Strukturfamilie zugehörig sind Isomere, die eine identische Struktur des Sauerstoffgerüsts aufweisen und sich, in Anlehnung an die Bernal-Fowler-Regeln [174], nur in der Position der Wasserstoffatome unterscheiden.

\subsubsection{Quantenchemische Rechnungen}

In Tabelle 6.1 sind die nach bisherigem Erkenntnisstand vier energetisch günstigsten Isomere und der Dodekaeder, welcher eine hohe Symmetrie aufweist, mit ihren durch MP2 berechneten Bindungsenergien unter Zuhilfenahme unterschiedlicher Basissätzen aufgelistet. Die relativen Stabilitäten sind aufgrund der verwendeten Methode in Kombination mit großen Basissätzen als verlässlich anzusehen [175 und beruhen auf Untersuchungen von Xantheas und Mitarbeitern [115, 172, 175]. Deutlich zu erkennen ist, dass das über die Kanten verbundene Prisma (Edgesharing), welches als Isomer A bezeichnet wird, in allen Fällen das energetisch Günstigste ist. Die Struktur ist in Abbildung 6.1 zu finden. Das dazugehörige Spektrum, berechnet mit der Rechenmethode MP2 unter Verwendung des aug-ccpVDZ Basissatzes, ist in Abbildung 6.2 dargestellt. Alle harmonisch berechneten IR-Wellenzahlen im Bereich der OH-Streckschwingung wurden mit dem Faktor 0,96 skaliert [115]. Im Bezug auf das idealisierte Sauerstoffgerüst hat Isomer A eine hohe Symmetrie. Acht der Wassermoleküle sind vierfach koordiniert (DDAA- 
Tabelle 6.1.: Berechnete MP2 Bindungsenergien in kcal/mol mit unterschiedlichen Basissätzen bis zum geschätzten CBS-Limit für die verschiedenen Isomere des $\left(\mathrm{H}_{2} \mathrm{O}\right)_{20}$ Clusters. Die Isomere sind von links nach rechts in aufsteigender Energie sortiert. Die Bestimmung der Nullpunktsenergie (ZPE) erfolgte aus Rechnungen mit dem aug-cc-pVDZ Basissatz. Die Energien von ${ }^{a}$ stammen aus $|172|$.

\begin{tabular}{|c|c|c|c|c|c|}
\hline Basis Satz & $\begin{array}{c}\text { Edge- } \\
\text { sharing }^{a} \\
\text { Isomer A }\end{array}$ & Drop-like & $\begin{array}{c}\text { Face- } \\
\text { sharing }^{a} \\
\text { Isomer C }\end{array}$ & $\begin{array}{l}\text { Fused } \\
\text { cubes }^{a}\end{array}$ & Dodecahedron $^{a}$ \\
\hline aug-cc-pVDZ & $-224,5$ & $-224,2$ & $-223,1$ & $-222,4$ & $-212,0$ \\
\hline aug-cc-pVTZ & $-220,3$ & $-219,1$ & $-218,5$ & $-217,9$ & $-209,3$ \\
\hline aug-cc-pVQZ & $-219,0$ & $-217,5$ & $-216,6$ & $-215,3$ & $-205,2$ \\
\hline CBS & $-217,9$ & $-216,3$ & $-215,0$ & $-212,6$ & $-200,1$ \\
\hline $\mathrm{CBS}(\mathrm{ZPE})$ & $(-163,1)$ & $(-162,6)$ & $(-160,1)$ & $(-157,5)$ & $(-147,9)$ \\
\hline
\end{tabular}

Motiv). Der charakteristische Peak dieses Isomers um $3150 \mathrm{~cm}^{-1}$ wird hervorgerufen durch die Oszillation von sechs Wassermolekülen, die als einfacher Donor und zweifacher Akzeptor (DAA-Motiv) fungieren. Die restlichen Moleküle sind DDAkoordiniert. Isomer B, welches als tröpfchenartig (Drop-like) bezeichnet wird, ist nahezu isoenergetisch zu A. Struktur B wurde als erstes von Furtado et al. entdeckt [176]. Die Energiedifferenz zwischen Isomer A und B beträgt im nullpunktskorrigierten CBS-Limit nur noch 0,5 kcal/mol. Das CBS-Limit wurde anhand der Werte aus den MP2-Rechnungen mit den Basisätzen aug-cc-pVDZ, aug-cc-pVTZ und aug-cc-pVQZ geschätzt. Die Nullpunktskorrektur erfolgte anhand einer harmonischen MP2-Rechnung auf aug-cc-pVDZ-Niveau. Isomer B besteht aus sechs DDAA Molekülen, von denen sich fünf auf der Oberfläche befinden. Vier dieser Moleküle binden im Inneren des Clusters ein Wassermolekül. Dieses ist in Abb. 6.1 blau gekennzeichnet. Die Oberfläche von Isomer B besteht aus vier viergliedrigen, sechs fünfgliedrigen und zwei sechsgliedrigen Ringen. Eine Hälfte besteht aus den miteinander verbundenen fünfgliedrigen Ringen, während die andere Hälfte aus ineinandergreifenden vier- und sechsgliedrigen Ringen gebildet wird. Das im IR-Spektrum bei kleinen Wellenzahlen für diesen Cluster charakteristische Signal (vgl. Abb. 6.2 besteht aus drei Peaks, welche von sieben DAA-Molekülen stammen. Die unterschiedlichen Positionen und Intensitäten der Peaks sind durch die unterschiedlichen Umgebungen der Moleküle begründet. Im Vergleich zu Isomer A 


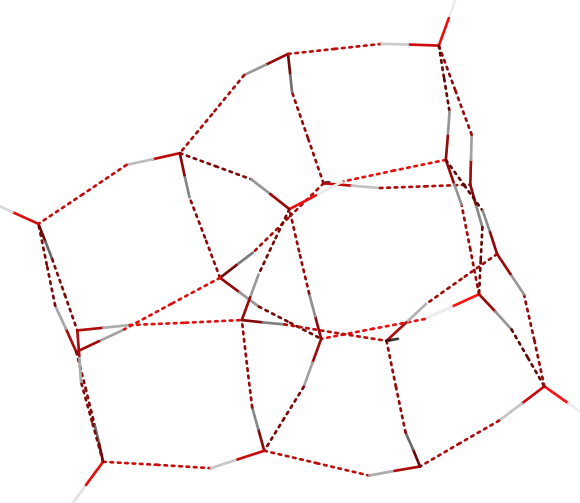

Isomer A Edge-sharing

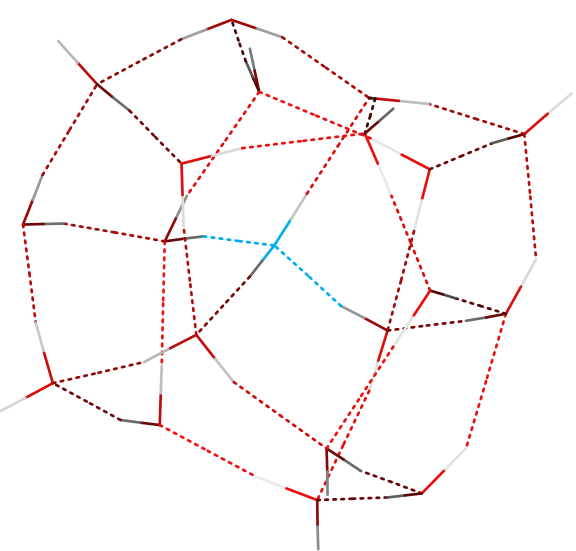

Isomer B Drop-like

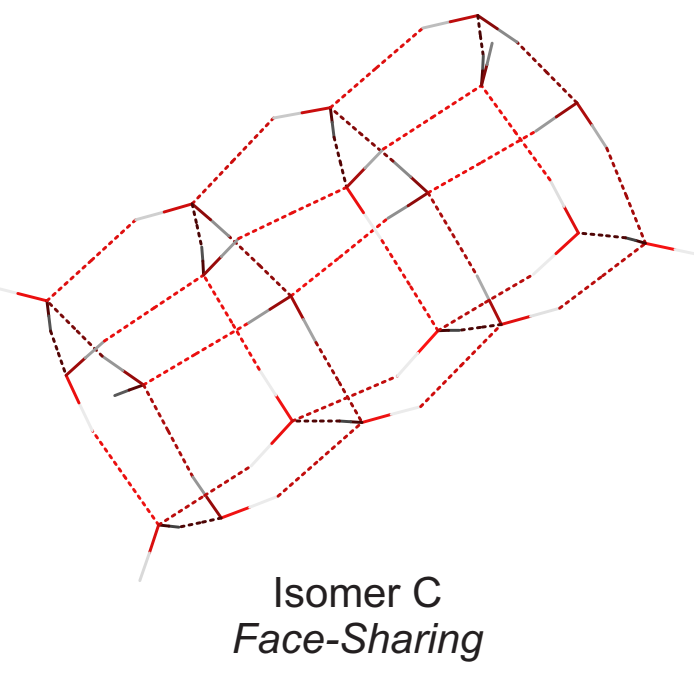

Abbildung 6.1.: Struktur der drei energetisch günstigsten $\left(\mathrm{H}_{2} \mathrm{O}\right)_{20}$-Isomere. Das vierfach koordinierte Wassermolekül im Inneren der Struktur B ist blau gekennzeichnet.

ist die dominante DAA-Bande rotverschoben. Die von Fanourgakis et al. diskutierten weiteren Isomerklassen, wie das über die Basisflächen verbundene Pentaprisma (Face-sharing), das in Anlehnung an $\left(\mathrm{H}_{2} \mathrm{O}\right)_{8}, 9,24,103$ aus Würfeln bestehende Aggregat (Fused cubes) oder das Dodekaeder haben geringere Bindungsenergien (vgl. Tab. 6.1) 172. Die Energiereihung ändert sich auch nicht durch größere Basissätze oder die Berücksichtigung der Nullpunktsenergie. Die Energiedifferenz vom Face-sharing Prisma, als Isomer C benannt, zum stabilsten Isomer A ist 

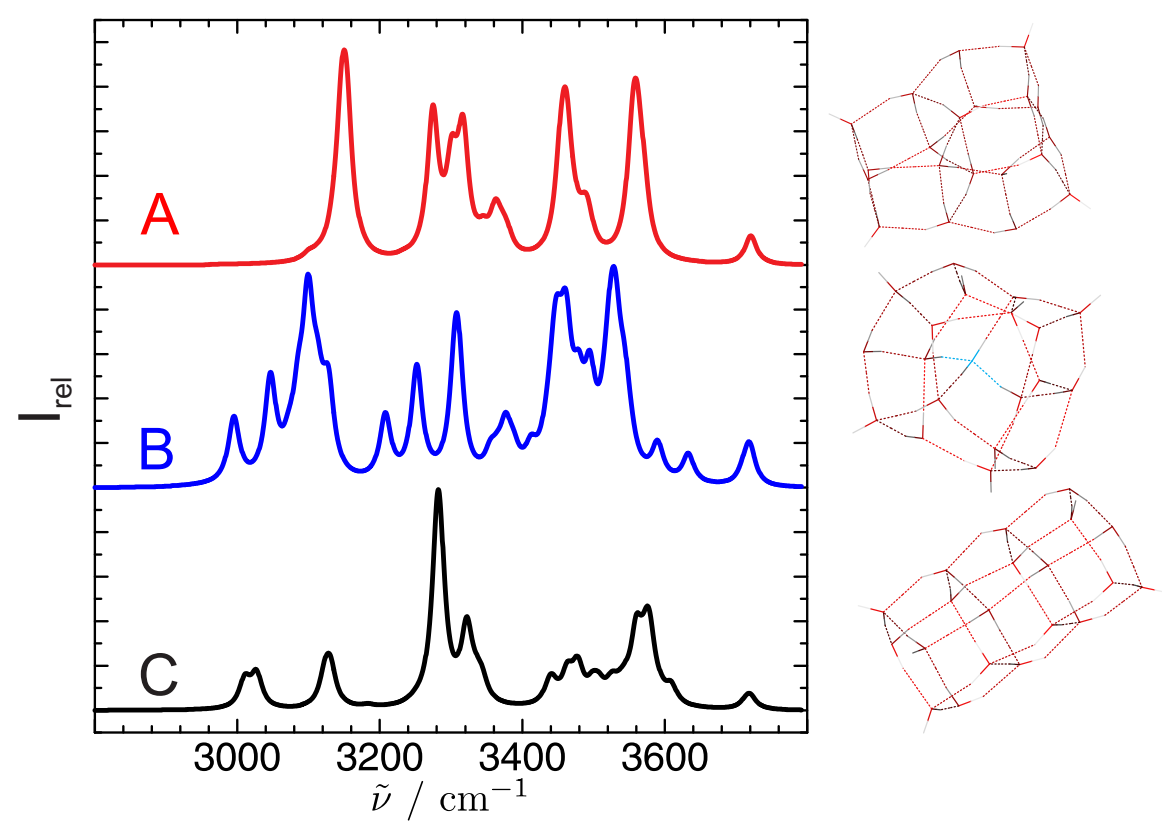

Abbildung 6.2.: Errechnetes Spektrum der OH-Streckschwingung der drei energetisch günstigsten $\left(\mathrm{H}_{2} \mathrm{O}\right)_{20}$-Isomere. Die Frequenzen sind mit MP2 unter Verwendung des Basissatzes aug-cc-pVDZ berechnet und mit dem Faktor 0,96 skaliert, sowie um $20 \mathrm{~cm}^{-1}$ LorentzVerbreitert.

$3 \mathrm{kcal} / \mathrm{mol}$ beim nullpunktskorrigierten CBS-Limit. Es besteht aus zwei fünfgliedrigen Ringen und fünfzehn viergliedringen Ringen auf der Oberfläche. Zehn der Wassermoleküle sind vierfach koordiniert und erzeugen im Spektrum einen dominanten Doppelpeak bei $3300 \mathrm{~cm}^{-1}$.

\subsubsection{Experimentelle Daten}

Die Arbeit von Buck et al. [39], die Masterarbeit von Pradzynski [47] und die Diplomarbeit von Zurheide $|79|$ zeigten, dass eine strukturelle Zuordnung bei IRSpektren unter Verwendung von Helium als Trägergas aufgrund breiter Absorptionsbanden nicht möglich ist. Falls die Ionisation zusätzlich mit 3,1 eV durchgeführt wird, kann das spektrale Signal durch Strukturen mit teilsolvatisiertem Natrium beeinflusst werden. Eine Möglichkeit der Signalverbesserung stellt die Mittelung über mehrere Messtage dar [80]. Abbildung 6.5 zeigt am Beispiel von $\mathrm{Na}\left(\mathrm{H}_{2} \mathrm{O}\right)_{20}$ in einer Neonexpansion, dass die Zusammenführung der Daten von neun Messtagen 


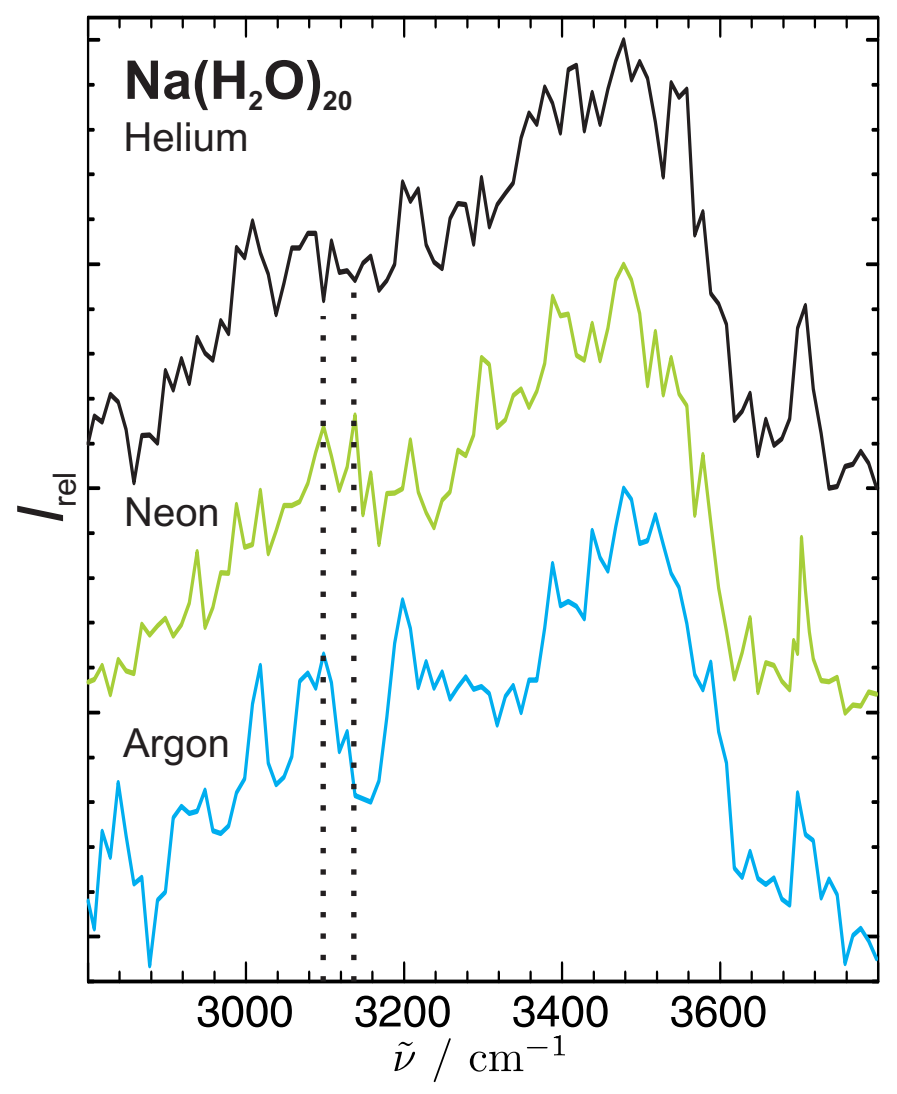

Abbildung 6.3.: Vergleich der experimentellen IR-Spektren von $\mathrm{Na}\left(\mathrm{H}_{2} \mathrm{O}\right)_{20}$ bei der Verwendung der unterschiedlicher Trägergase Helium, Neon und Argon. Die spektralen Daten sind über mehrere Messtage gemittelt.

zu einer Reduzierung des statistischen Rauschens führt. Eine ähnliche Gegenüberstellung fand für die IR-Spektren von $\mathrm{Na}\left(\mathrm{H}_{2} \mathrm{O}\right)_{20}$ in einer Argonexpansion statt und ist in Abbildung A.1 auf Seite Izu finden. Die Spektren jedes einzelnen Messtages unter Verwendung von Neon als Trägergas sind in Abb. A.2 im Anhang zu finden. Für die Gesamtmittelung wurde die Messtage 10.08.2012 und 30.05.2012 nicht aufgenommen, da das Spektrum eher dem Argonfall ähnelt. Werden diese beiden Tage trotzdem berücksichtigt, ändert sich die Gesamtabsorption kaun (vgl. Abb. A.3 auf Seite III.

Die IR-Spektren von $\mathrm{Na}\left(\mathrm{H}_{2} \mathrm{O}\right)_{20}$ unter Verwendung der Trägergase Helium, Neon und Argon sind in Abbildung 6.3 dargestellt. Das Absorptionssignal ist eine Mittelung aus den in Tabelle A.2 im Anhang aufgelisteten Messdaten für das jeweilige 
Trägergas. Für die Heliumexpansion wurde auf die Daten bei einem Stagnationsdruck von $3,9 \pm 0,1$ bar zurückgegriffen. Die Ionisation erfolgte wahlweise mit $360 \mathrm{~nm}(3,44 \mathrm{eV})$ oder 385-390 nm (3,22-3,18 eV). Das Absorptionsverhalten der Cluster bei den drei Bedingungen ist unterschiedlich, sie haben jedoch das gleiche Maximum bei $3480 \mathrm{~cm}^{-1}$. Das Maximum der freien OH-Streckschwingung liegt im Fall der Neon- und Argonexpansion bei $3703 \mathrm{~cm}^{-1}$. In der Heliumexpansion liegt die freie OH-Streckschwingung bei $3708 \mathrm{~cm}^{-1}$ und ist besonders stark ausgebildet. Unter Verwendung von Neon als Trägergas ist ein Doppelpeakmuster um $3100 \mathrm{~cm}^{-1} \mathrm{zu}$ erkennen. Die Stabilität dieses Doppelpeakmusters wird besonders deutlich in Abbildung 6.5. Diese enthält die Rohdaten vom 17.08.2012 im Vergleich zu zwei disjunkten Mittelungen dreier Messtage bzw. der Mittelung über alle Messtage.

Vergleicht man die gemittelten Spektren von $\left(\mathrm{H}_{2} \mathrm{O}\right)_{20}$ aus den Neonexpansionen mit den Spektren der benachbarten Clustergrößen $\left(\mathrm{H}_{2} \mathrm{O}\right)_{19}$ und $\left(\mathrm{H}_{2} \mathrm{O}\right)_{21}$ (s. Abbildung 6.4), so ist dieses Absorptionsdoppelmuster charakteristisch für $n=20$. Deutlich zu erkennen ist, dass in diesem Größenbereich eine strukturelle Zuordnung aufgrund der zahlreichen überlappenden Banden zwischen $3350 \mathrm{~cm}^{-1}$ und $3600 \mathrm{~cm}^{-1}$, also im DDAA- und DDA-Bereich, schwierig ist. Lediglich der DDA-Bereich bietet aufgrund seiner großen Aufspaltung zwischen $2900 \mathrm{~cm}^{-1}$ und $3150 \mathrm{~cm}^{-1}$ gute Ansatzpunkte. Der am meisten rotverschobene Peak bei $3100 \mathrm{~cm}^{-1}$ könnte auf Anwesenheit von Isomer B, der bei $3140 \mathrm{~cm}^{-1}$ auf die Anwesenheit von Isomer A hinweisen (vgl. Abb. 6.2), also den beiden als energetisch besonders günstig eingestuften Isomeren. Diese Hypothese soll im Folgenden untermauert werden.

\subsubsection{Vergleich der quantenchemischen und experimentellen Daten}

Die Gegenüberstellung von berechneten Spektren mit den experimentellen Daten erfolgt in Abbildung 6.6. Die beiden oberen Auftragungen vergleichen das in einer Neonexpansion generierte Spektrum mit einem aus den Isomeren A und B simulierten IR-Spektrum. Der Anteil der Isomere an den errechneten Intensitäten beträgt $65 \%$ an Isomer B und $35 \%$ an Isomer A, um den im Experiment beobachteten Doppelpeak im DAA-Bereich wiederzugeben. Das simulierte Spektrum 


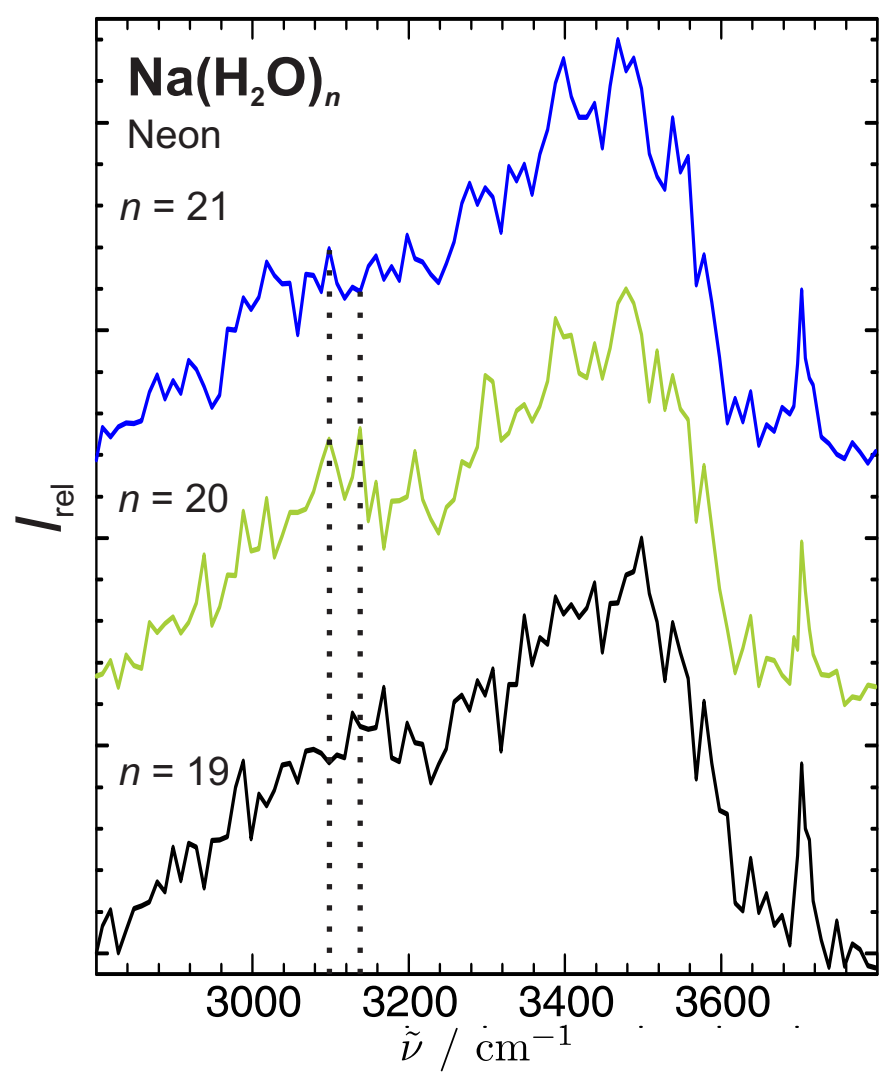

Abbildung 6.4.: Vergleich der experimentellen IR-Spektren von $\mathrm{Na}\left(\mathrm{H}_{2} \mathrm{O}\right)_{19,20,21}$ bei der Verwendung von Neon als Trägergas. Die Daten sind gemittelte Rohdaten aus neun Neonmessreihen.

ist im gesamten OH-Streckbereich in guter Übereinstimmung mit dem experimentellen. Die besonders ausgeprägten Merkmale des simulierten Spektrums, der Doppelpeak bei $3100 \mathrm{~cm}^{-1}, 3140 \mathrm{~cm}^{-1}$ und ein Peak bei $3310 \mathrm{~cm}^{-1}$, sind auch im experimentellen Spektrum zu beobachten.

Im Folgenden soll das in einer Argonexpansion erzeugte Spektrum diskutiert werden, da durch die Änderung der Expansionsbedingungen und des Trägergases sich die Population der Isomere ändern lässt 177]. Die beiden mittleren Spektren in der Abbildung 6.6 sollen dies demonstrieren. Das mit Argon bezeichnete Spektrum stellt eine Mittelung aus den Rohdaten vom 12.12.11, 20.12.11, 05.01.12 und 13.01.12 dar. Dieses Spektrum ist in guter Übereinstimmung mit dem simulierten Spektrum von Isomer B. Der gesamte DAA-Bereich kann durch Beiträge von Isomer B erklärt werden. Auch die Intensitäten werden bis auf die höchste Ban- 


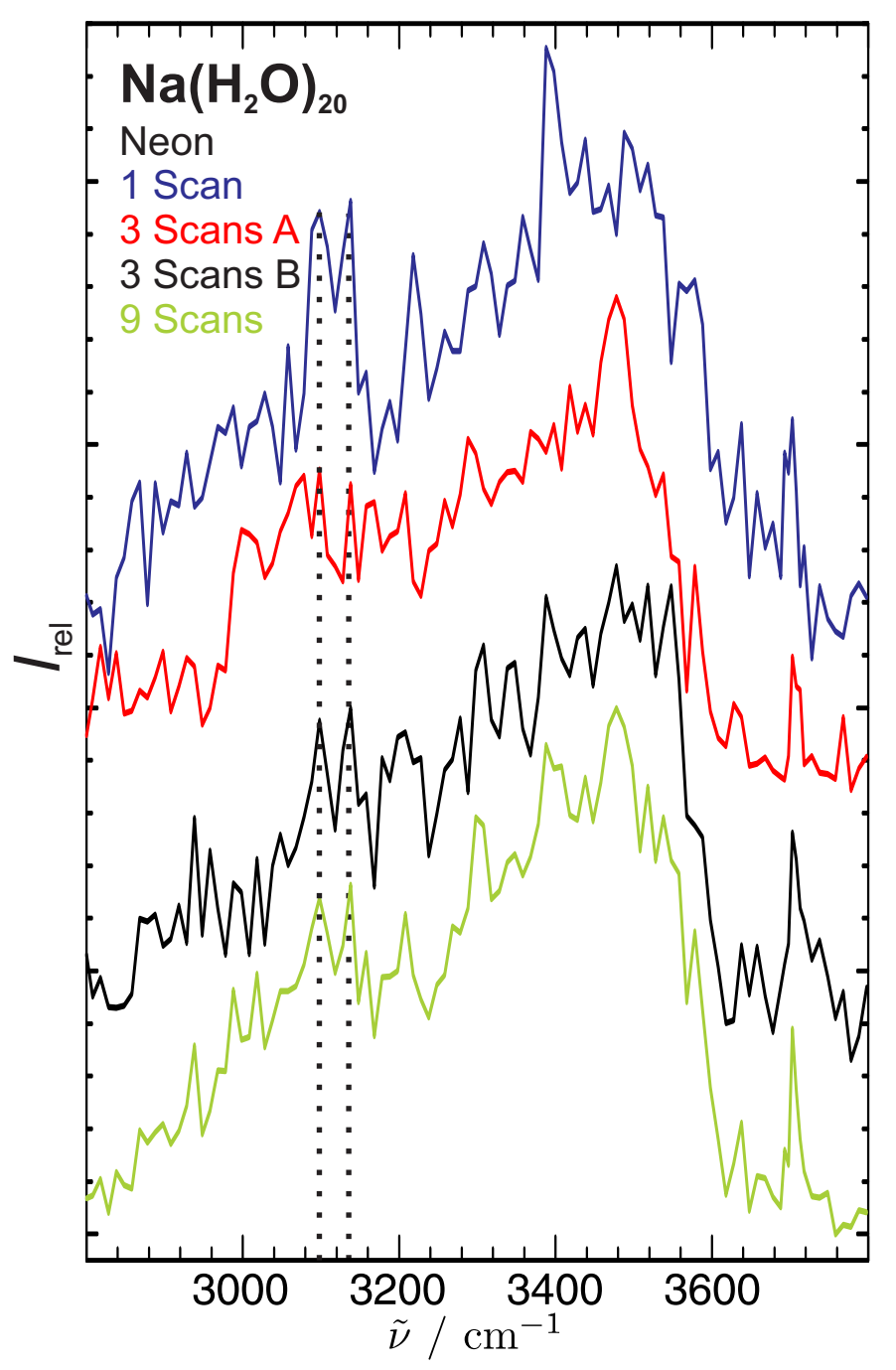

Abbildung 6.5.: Veranschaulichung des statistischen Rauschens für die IRSpektren von $\mathrm{Na}\left(\mathrm{H}_{2} \mathrm{O}\right)_{20}$. Das blaue Spektrum stellt eine einzelne Messung vom 17.08.2012, die weiteren Spektren stellen Mittelungen mehrere Messtage dar. In rot: 31.05.12, 13.07.12 und 15.08.12, in schwarz 30.05.12, 10.07.12 und 21.08.12, sowie in grün eine Mittelung aller Spektren.

de gut wiedergegeben. Es ist eine klare Separierung zwischen DAA-Oszillatoren unterhalb von $3180 \mathrm{~cm}^{-1}$ und DDAA-Oszillatoren oberhalb von $3200 \mathrm{~cm}^{-1} \mathrm{zu}$ beobachten. Die hohe Intensität um $3200 \mathrm{~cm}^{-1}$, bzw. der Intensitätseinbruch um $3160 \mathrm{~cm}^{-1}$ ist vermutlich auf eine Fermiresonanz dieses DDAA-Motivs mit der OH-Biegeschwingung zurückzuführen, die von Otto et al. 15 für kleine Was- 


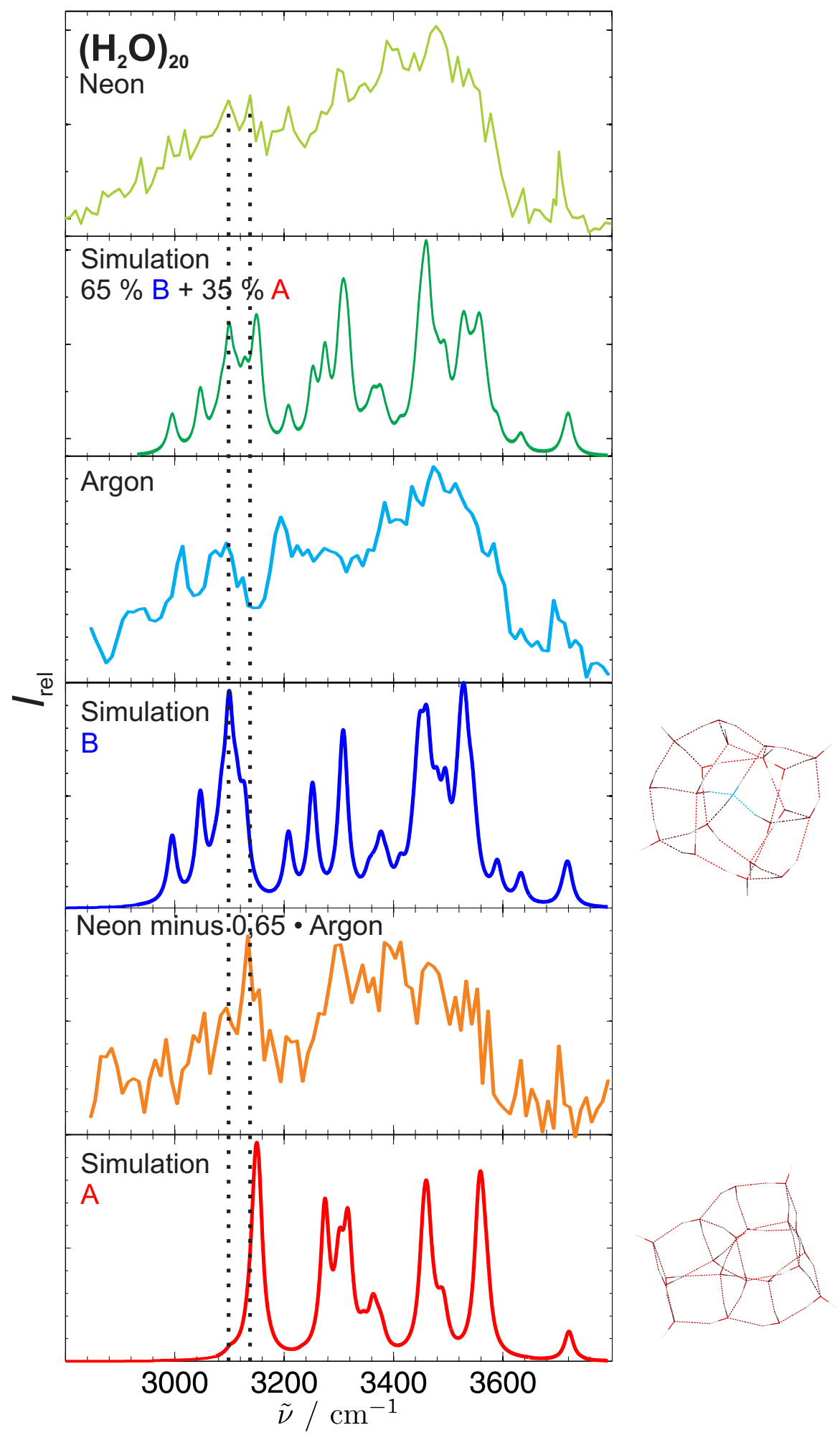

Abbildung 6.6.: Vergleich der gemessenen und berechneten Spektren. Erläuterungen im Text. 
sercluster nachgewiesen wurde. Dieser Effekt tritt ebenfalls im bereits gezeigten Spektrum von $\left(\mathrm{H}_{2} \mathrm{O}\right)_{113 \pm 13}$ des als kalt klassifizierten Expansionsfalls auf (vgl. Abb. 5.9 auf Seite 101). Im Experiment scheint die Aufspaltung der DDA-Motive größer zu sein, als die harmonisch berechneten Frequenzen vorhersagen. Das Fehlen von Isomer A im Argonspektrum könnte auf eine höhere Stabilität von Isomer B deuten. Kinetische Effekte können jedoch nicht ausgeschlossen werden. Eventuell führen Expansionen mit Neon als Trägergas zu einer besseren Relaxation der Cluster in ihr thermodynamisches Minimum, wie es auch schon für anionische Cluster beobachtet wurde [177]. Die Temperatur der Cluster in der Neonexpansion wurde mit Hilfe von Simulationen von Gimelshein und Mitarbeitern auf ca. $100 \mathrm{~K}$ geschätzt [178]. Die Expansion mit Argon führt wahrscheinlich aufgrund der größeren Masse des Stoßpartners und dem größeren Anteil an Trägergas von ca. $80 \%$ für Argon gegenüber ca. 57\% für Neon zu kälteren Clustern [11.

Die beiden unteren Spektren der Abbildung 6.6 stellen das Differenzspektrum der Messungen mit Neon und Argon als Trägergas dem berechneten Spektrum von Isomer A gegenüber. Beide Spektren zeigen das gleiche charakteristische Absorptionsverhalten im gesamten OH-Streckbereich mit einen Peak im DAA-Bereich bei $3140 \mathrm{~cm}^{-1}$ mit einer Lücke zu den DDAA-Oszillatoren, die um $3300 \mathrm{~cm}^{-1}$ absorbieren. Die Übereinstimmungen des Differenzspektrums mit den Hauptmerkmalen des simulierten Spektrums von Isomer A sind ein starker Hinweis auf die Präsenz von diesem Isomer in der Expansion mit Neon als Trägergas. Da Isomer A im Bezug auf das Sauerstoffgerüst eine hohe Symmetrie aufweist, sollte das IR-Spektrum empfindlich gegenüber Störungen durch das Natrium aufgrund des Symmetriebruchs sein. Um dieses zu testen, wurden Rechnungen mit natriumdotierten Varianten mit Hilfe der Methode B3LYP unter Verwendung des Basissatzes 6-31+G** mit dem Programmpaket Gaussian 09 [114] durchgeführt. Die Natriumanlagerung mit Ringöffnung wurde an verschiedenen Positionen von Isomer A durchgeführt. In Abbildung 6.7 ist das stabilste Isomer dieser Testreihe abgebildet. Das Natrium bindet an zwei Wassermoleküle, sodass im Cluster ein DDAA-Molekül zum DDAC und ein DDA-Molekül zum DAC wird. Die Störung hat zudem Einfluss auf die kooperativen Effekte in den Ringen. Das dazugehörige Spektrum mit und ohne Natrium im Vergleich zum Differenzspektrum von Neon und Argon ist in Abbildung $6.8 \mathrm{zu}$ finden. Die Frequenzen wurden, aufgrund der geänderten Methode, um den Faktor 0,965 skaliert und auf den Maximalwert normiert. Die Natriumstörung be- 


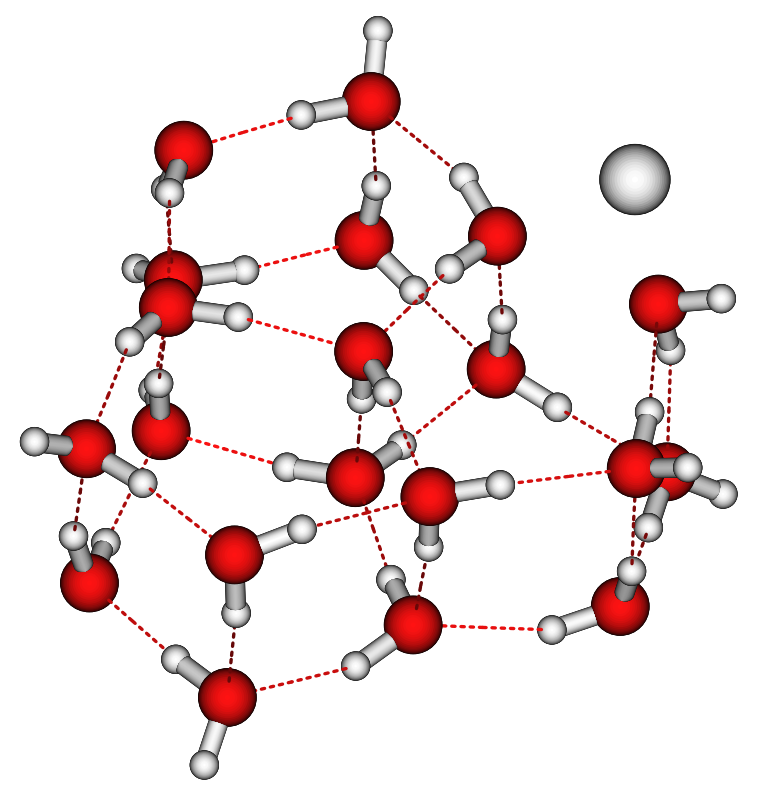

Abbildung 6.7.: Struktur des stabilsten Isomers mit Natriumanlagerung. Die Geometrieoptimierung von Ausgangsstruktur und Endstruktur erfolgte über Gaussian 09 114 mit Hilfe der B3LYP-Methode unter Verwendung des 6-31+ $\mathrm{G}^{* *}$ Basissatzes.

wirkt eine Verringerung der Intensität und eine Aufspaltung der DAA-Bande um $3140 \mathrm{~cm}^{-1}$, was das Absorptionsverhalten sowohl des Differenzspektrums als auch des Neon-Spektrums um $3000 \mathrm{~cm}^{-1}$ wiedergibt. Das Motiv um $3300 \mathrm{~cm}^{-1}$ wird blauverschoben. Dieser Natrium-Sonden-Effekt könnte die breite Lücke zwischen DAA- und DDAA-Motiv im Differenzspektrum erklären. Die anderen Motive gewinnen relativ zum ungestörten Cluster an Intensität und die Lücke zwischen DDAA- und DDA-Motiv um $3400 \mathrm{~cm}^{-1}$ wird geschlossen. Die Aufspaltung der freien OH-Schwingung ist im Differenzspektrum und im simulierten Spektrum zu beobachten.

\subsection{Clustergröße $n=25$}

Anders als im Fall von $\left(\mathrm{H}_{2} \mathrm{O}\right)_{20}$ sind bei anderen, vor allem größeren Wasserclustern, die relativen Stabilitäten der möglichen Isomere deutlich schlechter charakterisiert. Eine versuchsweise Strukturzuordnung für die Clustergröße $n=25$ erfolgte in Zusammenarbeit mit der Gruppe von Prof. Dr. Bernd Hartke von der Christian- 


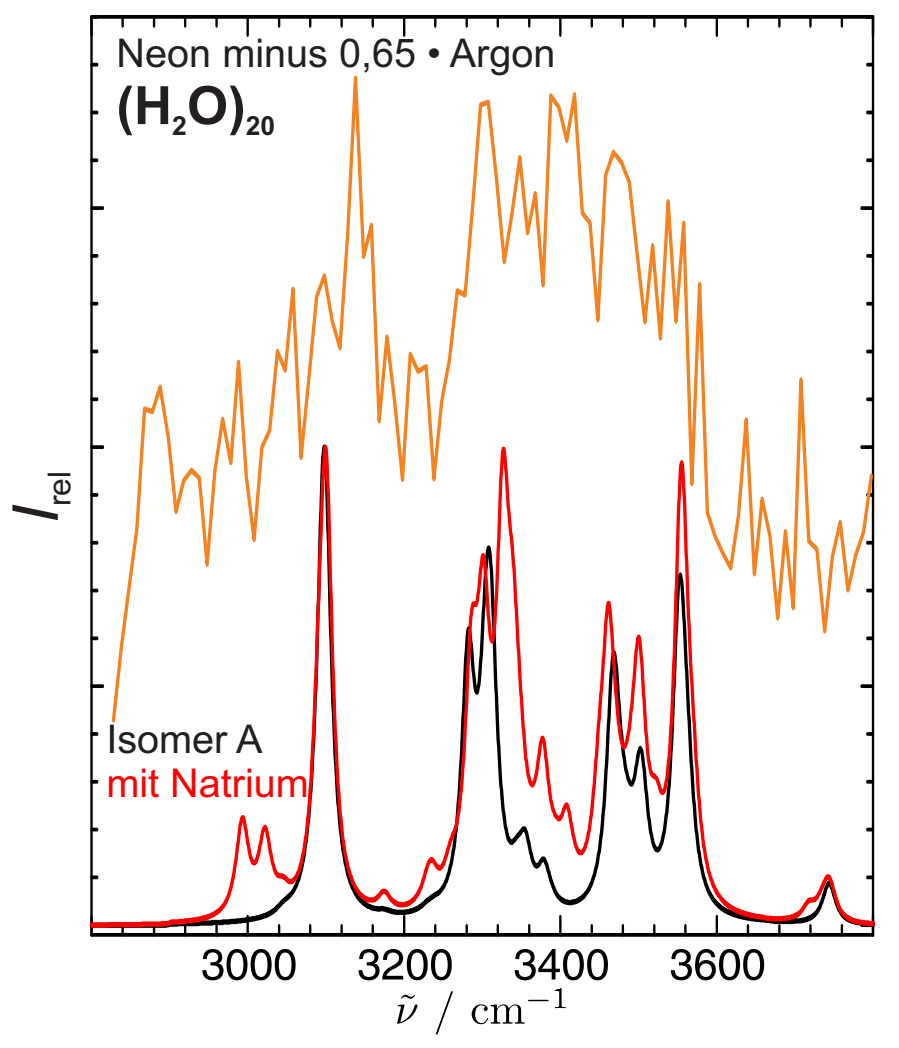

Abbildung 6.8.: Vergleich zwischen dem Differenzspektrum von Neon und Argon und einem harmonisch berechneten Spektrum von Isomer A mit (in rot) und ohne (in schwarz) Natrium. Für die Berechnung wurde hier die Methode B3LYP mit dem Basissatz $6-31+\mathrm{G}^{* *}$ unter Zuhilfenahme des Programmpakets Gaussian 09 [114] verwendet. Die Frequenzen wurden mit dem Faktor 0,965 skaliert und $20 \mathrm{~cm}^{-1}$ Lorentz-verbreitert, die Intensitäten sind auf den maximalen Wert normiert.

Albrechts-Universität Kiel und Dr. Johannes M. Dieterich. Für die Erzeugung und Optimierung der Strukturen wurde ein genetischer Algorithmus [179] auf Basis des TTM3-F-Potentials [120] verwendet. Es wurden 100 Isomere für diese Clustergröße erzeugt und nach ihrer Energie sortiert. Duplikate wurden eliminiert und eine Boltzmann-Gewichtung in dem Temperaturbereich des Experiments von $100 \mathrm{~K}$ durchgeführt. Die Berechnung der IR-Spektren erfolgte mit dem anharmonischen Modell von Victoria Buch, welches bereits gute Ergebnisse in der strukturelle Bestimmung der Clustergrößen $n=7-10$ erzielt hat 24, 151]. Die Zuordnung von simulierten und experimentellen Spektren erfolgte visuell auf Basis der energetisch 
günstigsten Isomere in einem Energiefenster von $10 \mathrm{~kJ} / \mathrm{mol}$ [1].

Die experimentellen Spektren sind in guter Übereinstimmung mit dem simulierten Spektrum, welches auf der Absorption eines Isomers basiert (vgl. Abb. 6.10). Dessen Struktur ist aus zwei unterschiedlichen Perspektiven in Abbildung 6.9 gezeigt. Der Cluster besteht hauptsächlich aus vier- und fünfgliedrigen Ringen. Anders als
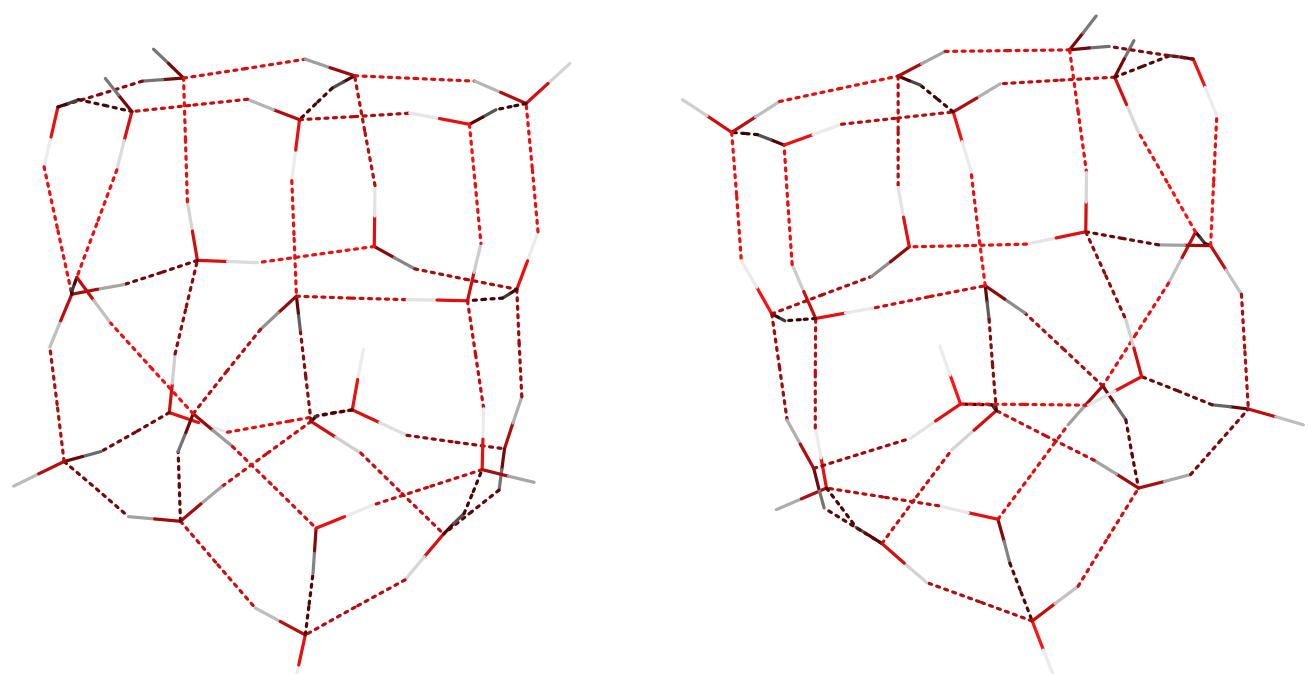

Abbildung 6.9.: Struktur des $\left(\mathrm{H}_{2} \mathrm{O}\right)_{25}$-Isomers aus zwei verschiedenen Perspektiven, entnommen aus 11 .

bei Isomer B für $n=20$ liegen alle Wassermoleküle auf der Oberfläche des Clusters. Es sind 13 Moleküle vierfach koordiniert, jeweils 6 weisen ein DDA- bzw. DAAMotiv auf. In dem oben genannten Energiefenster sind 18 Strukturen enthalten, von denen die meisten ein internes Wassermolekül besitzen, ein Isomer weist bereits zwei interne Wassermoleküle auf [1].

In Abbildung 6.10 ist das simulierte Spektrum den in Neon und Argon erzeugten experimentellen Spektren sowie einer Mittelung des Gesamtsignals der beiden Fälle gegenübergestellt. Die Spektren stellen gemittelte Rohdaten aus neun (31.05.2012, 10.07.2012, 12.07.2012, 13.07.2012, 15.08.2012, 16.08.2012, 17.08.2012, 21.08.2012 und 22.08.2012) bzw. vier Messreihen (12.12.2011, 20.12.2011, 05.01.2012 und 13.01.2012) dar. Beide experimentelle Spektren weisen einen beinahe identischen Absorptionsverlauf mit breiten Peaks um $3100 \mathrm{~cm}^{-1}$, $3200 \mathrm{~cm}^{-1}$ und $3300 \mathrm{~cm}^{-1}$ auf. In Neon hat die freie OH-Streckschwingung eine höhere Intensität. Die von Hartke vorgeschlagene Struktur scheint das Absorpti- 


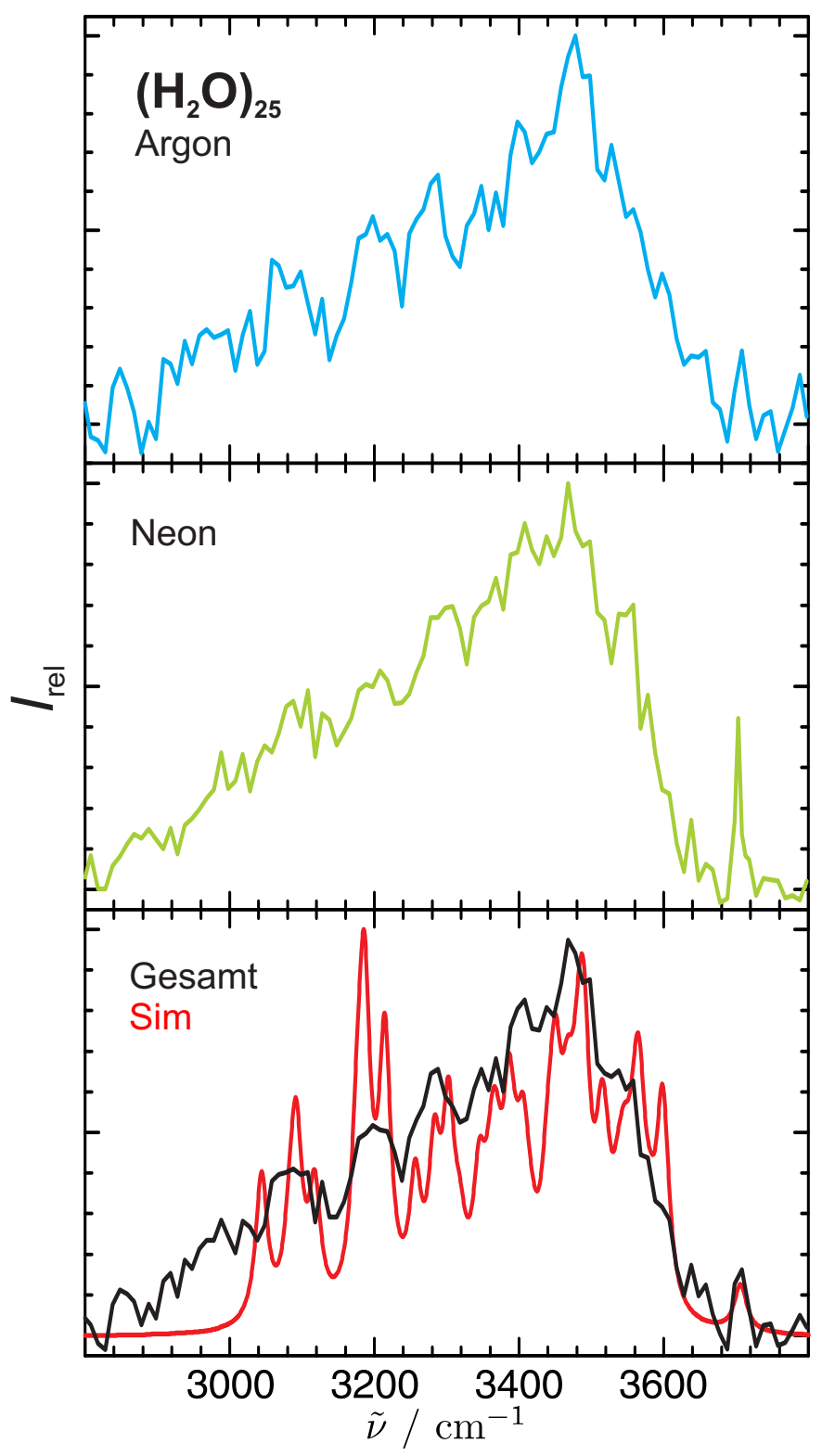

Abbildung 6.10.: Vergleich zwischen simuliertem IR-Spetrum des $\left(\mathrm{H}_{2} \mathrm{O}\right)_{25}$ Clusters (rot, $20 \mathrm{~cm}^{-1}$ Lorentz-Verbreiterung) und experimentellen IR-Spektren des $\mathrm{Na}\left(\mathrm{H}_{2} \mathrm{O}\right)_{25}$ Clusters. Die experimentellen Spektren stellen eine Mittelung aus neun Neon (in gün), vier ArgonMessreihen (in blau) sowie eine Mittelung aus beiden Fällen dar.

onsverhalten des experimentell erhaltenen Spektrums für $\left(\mathrm{H}_{2} \mathrm{O}\right)_{25}$ gut wiederzugeben. Es könnte aber auch durch eine Überlagerung mehrerer Isomere hervorgerufen werden. 


\subsection{Clustergröße $n=32$}

Die Suche nach möglichen Isomeren für die Beschreibung des experimentellen Spektrums der Clustergröße $n=32$ erfolgte analog zum vorherigen Fall in Kooperation mit der Gruppe von Prof. Dr. Bernd Hartke und Dr. Johannes M. Dieterich. Für eine relativ gute Übereinstimmung zwischen experimentellem und simuliertem Spektrum in Abbildung 6.12 sind sieben der 38 Isomere in dem Energiefenster von $10 \mathrm{~kJ} / \mathrm{mol}$ nötig. Alle Isomere weisen ähnliche Strukturen auf und unterscheiden
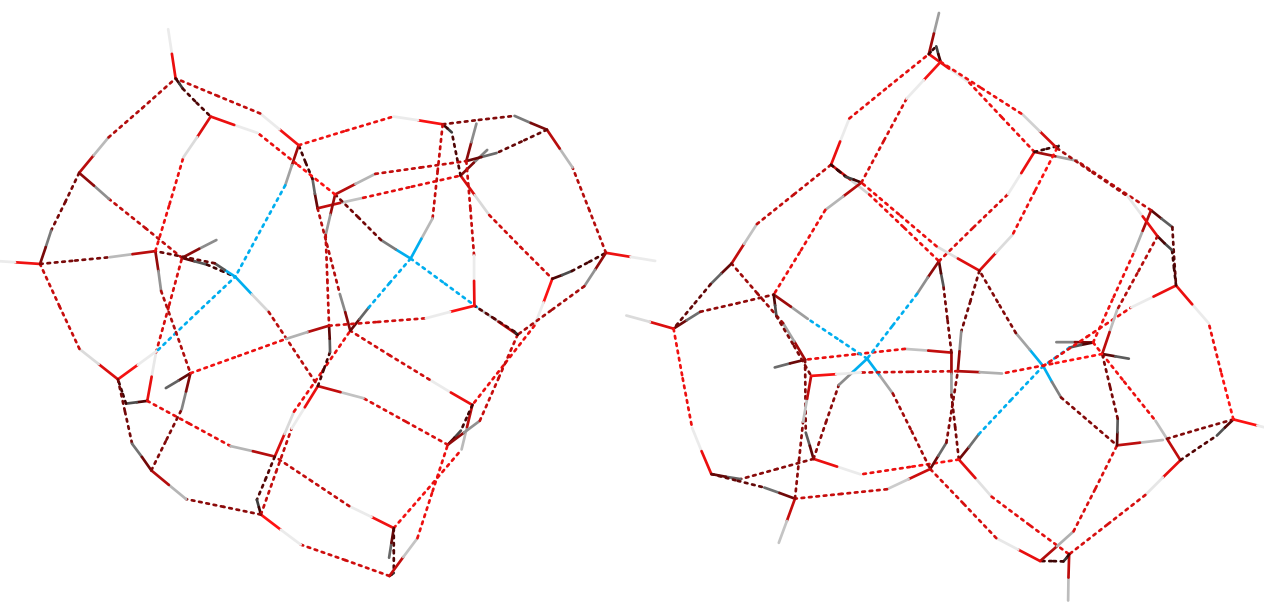

Abbildung 6.11.: Eine Struktur der für die Simulation des IR-Spektrums beteiligten $\left(\mathrm{H}_{2} \mathrm{O}\right)_{32}$-Isomere aus zwei verschiedenen Perspektiven, entnommen aus |11|.

sich nur in den Feinheiten 11. Eines der für das simulierte Spektrum verwendeten Isomere ist in Abbildung 6.11 aus zwei Perspektiven dargestellt. Von den 18 vierfach koordinierten Molekülen befinden sich zwei im Inneren des Clusters (blau markiert). Jeweils sieben Moleküle weisen eine DDA- bzw. DAA-Koordination auf. Der Cluster ist hauptsächlich aus vier- und fünfgliedrigen Ringen aufgebaut. Die experimentellen Spektren unter Verwendung der Trägergase Neon und Argon zeigen gute Übereinstimmungen (vgl. Abbildung 6.12. Das Signal-zu-RauschVerhältnis ist allerdings im Argon-Fall deutlich schlechter. Lediglich die Absorptionsbande um $3200 \mathrm{~cm}^{-1}$ sticht hier hervor, die wie in den vorherigen Fällen wahrscheinlich durch anharmonische Effekte, wie z.B eine Fermiresonanz mit dem Oberton der Knickschwingung, bedingt sein kann [15]. Das simulierte Spektrum gibt bis auf Feinheiten das Absorptionsmuster des Gesamtsignals wieder. Auch 


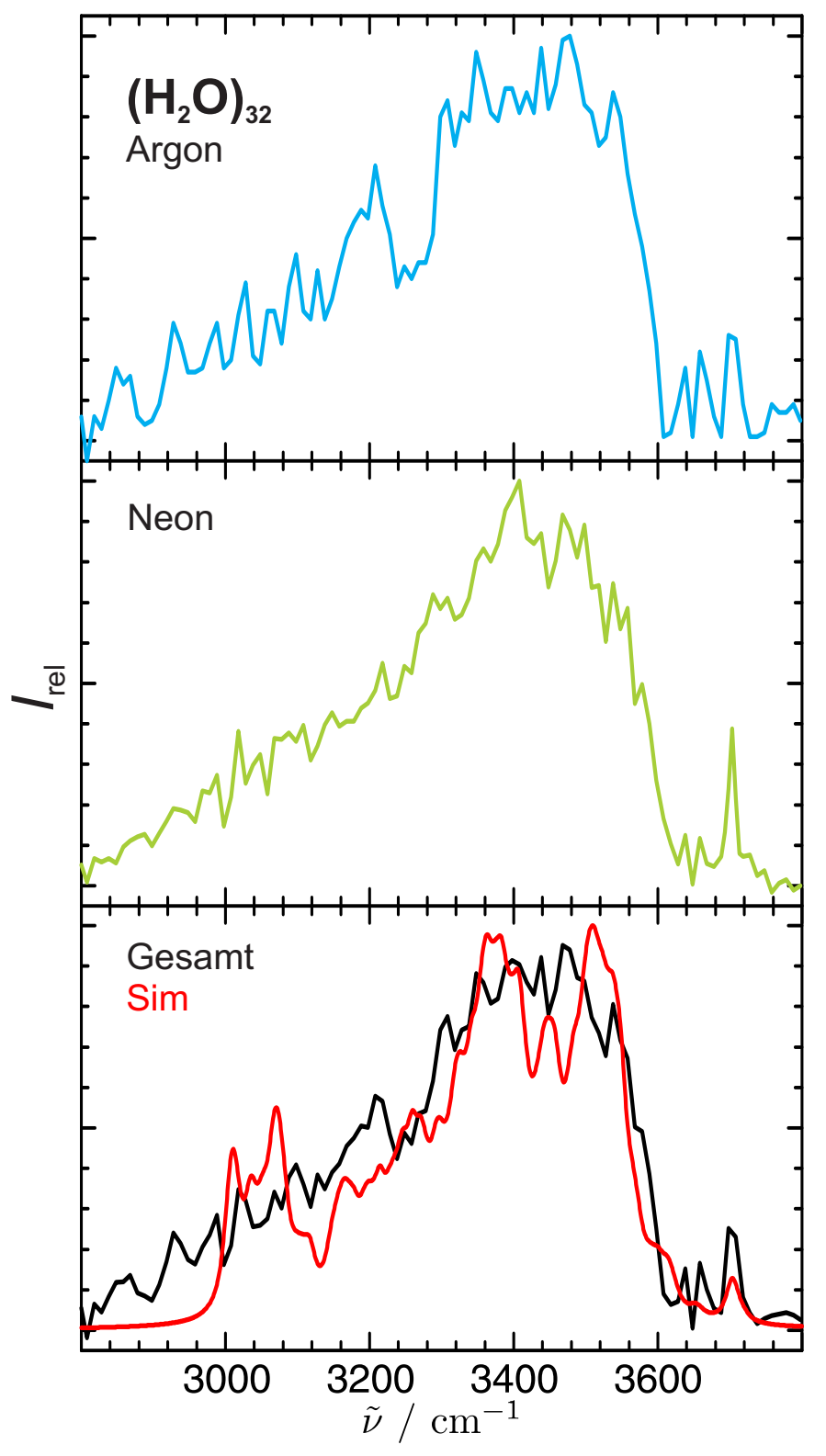

Abbildung 6.12.: Vergleich zwischen simuliertem IR-Spektrum des $\left(\mathrm{H}_{2} \mathrm{O}\right)_{32}$ Clusters (rot, $20 \mathrm{~cm}^{-1}$ Lorentz-Verbreiterung) und experimentellen IR-Spektren des $\mathrm{Na}\left(\mathrm{H}_{2} \mathrm{O}\right)_{32}$ Clusters. Die experimentellen Spektren stellen eine Mittelung aus neun Neon (in gün), vier ArgonMessreihen (in blau), sowie eine Mittelung aus beiden Fällen dar.

wenn es nicht möglich ist bei dieser Clustergröße das experimentelle Spektrum durch ein einzelnes oder einige wenige Isomere $\mathrm{zu}$ beschreiben, so unterscheidet 
sich die generelle Struktur der gefundenen Isomere kaum [11.

\subsection{Zusammenfassung und Zwischenfazit}

Die Analyse der Clustergrößen $n=20, n=25$ und $n=32$ hat gezeigt, dass durch die IR-CIPI-Methode eine Untersuchung der Strukturen neutraler Wassercluster anhand ihres IR-Spektrums im OH-Streckschwingungsbereich über den vorher bekannten Größenbereich von $n=6$ bis 10 hinaus möglich ist 24,151|. Im Hinblick auf die seit zwei Jahrzehnten anhaltende Diskussion über die Strukturen der Clustergröße $n=6$ [17,68 70, 180|, sind die hier gezeigten Strukturen als erste Deutung des experimentellen Befunds zu verstehen. Die Zuordnungen sind dabei auf zwei unterschiedliche Arten zustande gekommen.

Das Beispiel der Clustergröße $n=20$ zeigt, dass eine Strukturzuordnung möglich ist, wenn die Theorie weit genug vorangeschritten ist. Bei dieser Clustergröße konnte auf einen großen Pool an Strukturklassen zurückgegriffen werden, deren Bindungsenergien bis zu einem sehr hohen Rechenniveau bekannt sind [73,74, 172, 173]. Doch erst die Entdeckung einer neuen Isomerklasse mit einem internen Wassermolekül in Verbindung mit den unterschiedlichen experimentellen Befunden unter Verwendung von Neon und Argon als Trägergas ermöglichte eine konsistente Erklärung des experimentellen Befunds 115.

Die Strukturuntersuchung der Clustergrößen $n=25$ und $n=32$ erfolgte mit Hilfe eines genetischen Algorithmus [179] auf Basis des TTM3-F-Potentials [120. Die erste Analyse kann allerdings keinen Anspruch auf Strukturaufklärung stellen. Die IR-Spektren im OH-Streckbereich der in einem Energiefenster von $10 \mathrm{~kJ} / \mathrm{mol}$ stabilsten Isomere wurden mit Hilfe des anharmonischen Modells von Victoria Buch 24, 151] erzeugt und visuell mit den experimentellen Spektren verglichen. Dieses Vorgehen ist besonders effizient bei der Untersuchung größerer Cluster, da auf zeitintensive, harmonische oder anharmonische DFT-Berechnungen oder die noch zeitintensiveren $a b$ initio Methoden, die beide eine zusätzliche Optimierung benötigen, verzichtet werden kann und das Spektrum innerhalb von Sekunden für das jeweilige Isomer vorliegt [11]. Das Energiefenster von $10 \mathrm{~kJ} / \mathrm{mol}$ soll mögliche Effekte der ZPE und der Entropie durch die Clustertemperatur mit berücksichtigen, da diese die Energiereihung der erhaltenen Strukturen verändern können. 
Für die Clustergröße $n=25$ konnte ein Isomer mit einer "all-surface" Topologie den experimentellen Absorptionsverlauf wiedergeben. Die von Sahu et al. in einer aktuellen Studie publizierten Minima für $\left(\mathrm{H}_{2} \mathrm{O}\right)_{25}$ auf Basis von Rechnungen mit MP2/aug-cc-pVDZ zeigen keine Übereinstimmungen mit dem hier vorliegenden experimentellen Spektrum [181]. Falls neben den dort aufgeführten Isomeren noch weitere vorliegen sollten, wäre für den $\left(\mathrm{H}_{2} \mathrm{O}\right)_{25}$-Cluster eine strukturelle $\mathrm{Zu}$ ordnung schwer möglich. Für die Clustergröße $n=32$ geben sieben strukturähnliche Isomere den experimentellen Absorptionsverlauf wieder.

Die Diplomarbeit von Zurheide [79 und die Masterarbeit von Dierking [80 haben sich mit der Entwicklung der im Rahmen dieser Arbeit gemessenen IR-Spektren im Größenbereich bis $n \leq 100$ beschäftigt. Eine kurze Zusammenfassung soll anhand von Abbildung 6.13 vorgestellt werden [182. Diese enthält im oberen Drittel die vierfach geglätteten Konturplots der gemittelten Neonmessreihen im Größenbereich von $n=10-80$. Die Glättung erfolgte dreifach für die Wellenlänge und einfach für die Clustergröße. In der Mitte ist ein Auschnitt aus dem Absorptionsbereich der freien OH-Streckschwingung in einem Konturplot abgebildet. Für diese Auftragung erfolgte keine Glättung der Daten, jedoch wurde die maximale IR-Absorption auf ein Gesamtintegral von 1 normiert. Zudem sind die Spektren mit einer Auflösung von $5 \mathrm{~cm}^{-1}$ aufgenommen. Im unteren Bereich ist eine Auftragung der Quotienten der Spektralbereiche für DDAA- (3248$\left.3378 \mathrm{~cm}^{-1}\right)$ und DAA-Banden $\left(2898-3078 \mathrm{~cm}^{-1}\right)$ für neun gemittelte Neon- bzw. vier gemittelte Argon-Messreihen abgebildet. Deutlich zu erkennen ist, dass die Anzahl an DDAA-Molekülen im Verhältnis zu den DAA-Molekülen ansteigt. Im IR-Spektrum über den gesamten OH-Streckbereich wird dies durch eine Zunahme der Absorption um $3400 \mathrm{~cm}^{-1}$ deutlich. Mit zunehmender Clustergröße nähert sich das Absorptionsverhalten dem der amorphen Cluster an. Die Intensität der freien OH-Streckschwingung oszilliert sehr stark im betrachteten Clusterbereich. Die zwischen $n=19$ und $n=40$ zu beobachtende Rotverschiebung der Absorptionsbande wurde schon von Fujii und Mitarbeitern beschrieben [37. Dieser Befund konnte mit der hier in der Arbeit verwendeten Methode bestätigt werden. Vergleicht man Auffälligkeiten im Spektrum und Ausreißer im Verhältnis der Absorption von DDAA zu DAA, so liegen Besonderheiten bei einigen Clustergrößen vor, die ein ähnliches Muster aufweisen. Auffällig ist ein im Vergleich zu den benachbarten Clustergrößen höherer Anteil an DDAA-Molekülen, die eine stärkere 


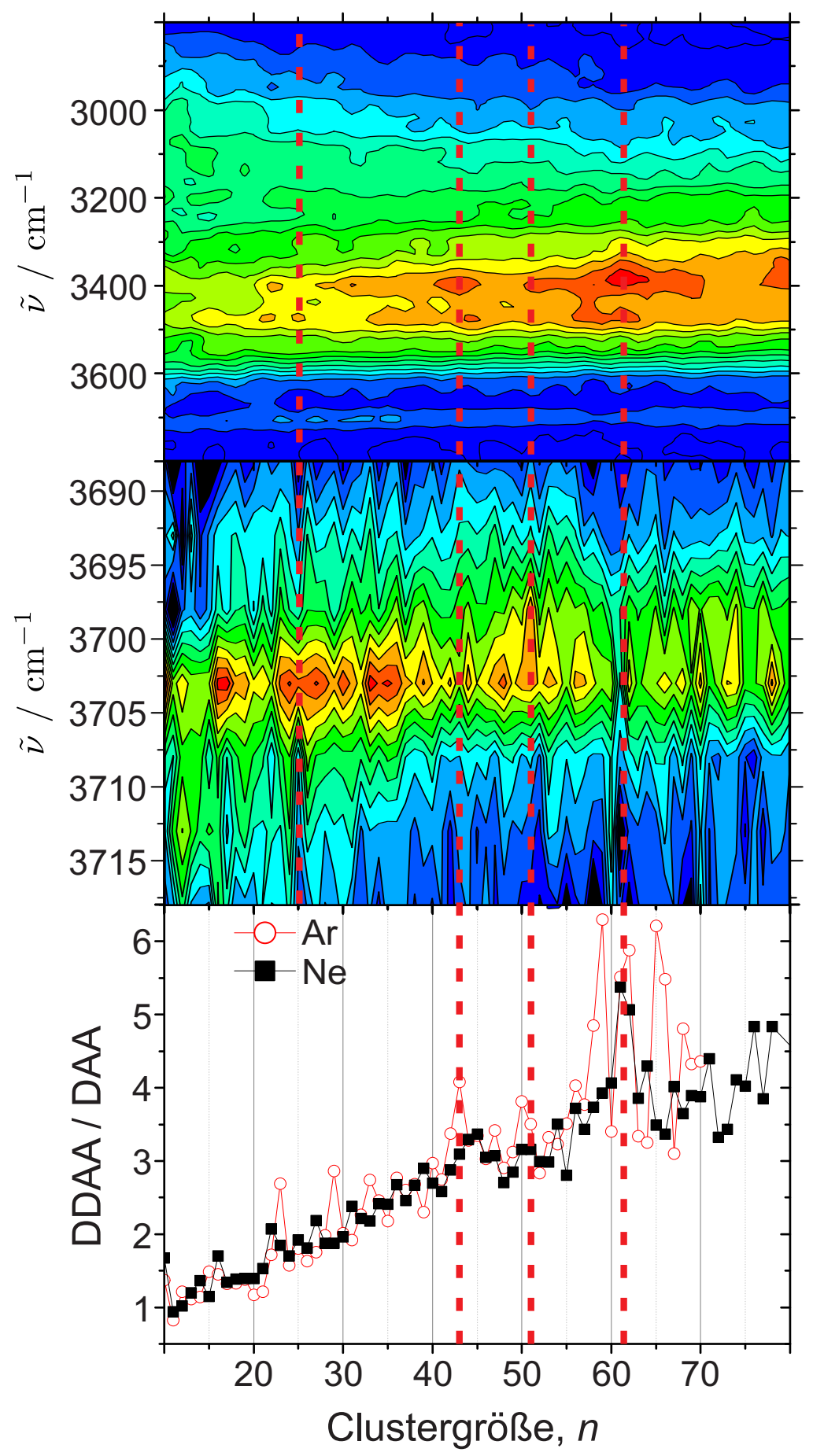

Abbildung 6.13.: Oben: Konturplot des gesamten Absorptionsbereichs für $\mathrm{Na}\left(\mathrm{H}_{2} \mathrm{O}\right)_{10-80}$ des Neon-Falls; Mitte: Konturplot der freien $\mathrm{OH}-$ Streckschwingung für $\mathrm{Na}\left(\mathrm{H}_{2} \mathrm{O}\right)_{10-80}$ des Neon-Falls; Unten: Auftragung des Quotienten der Integrale der Spektralbereiche für DDAA- und DAA-Banden für Neon und Argon. Weitere Erläuterungen im Text. Adaptiert nach $[80 \mid$ und $\mid 182]$. 
Absorption um $3400 \mathrm{~cm}^{-1}$ und eine schwächere Absorption bei der freien $\mathrm{OH}-$ Streckschwingung verursachen. $\mathrm{Zu}$ beobachten ist dieses Verhalten insbesondere bei den Clustergrößen $n=43$ und $60 / 61$. Zusätzlich weist die Gesamtabsorption einen einzelnen Peak bei den Clustergrößen $n=25,44$ und $60 / 61 \mathrm{um} 3470 \mathrm{~cm}^{-1}$ auf.

Relativ zu ihren Nachbarn tritt eine Rotverschiebung der freien $\mathrm{OH}-$ Streckschwingung bei Clustern mit ungerader Wassermolekülanzahl in dem Bereich $n=49-55$ auf und ist besonders stark hinsichtlich der Intensität für $\mathrm{Na}\left(\mathrm{H}_{2} \mathrm{O}\right)_{51}$ ausgeprägt. Die Anomalie dieses Clustergrößenbereichs wurde in FTICR Studien an protonierten Wasserclustern aus der Gruppe von Beauchamp [183] und bei Messungen des Photoelektronenspektrums anionischer Wassercluster von Ma et al. beobachtet [95]. Nur für die Clustergrößen $n=51$ und 53 fanden Ma et al. die Signatur eines Elektrons, das auf der Oberfläche der Cluster gebunden ist. Bei den anderen Clustergrößen ist dieses Signal von den solvatisierten Elektronen überlagert. Dies könnte auf einen besondere Stabilität der neutralen Wassercluster dieser Größe deuten, wie sie auch von Kazachenko und Thakkar 75 in ihren quantenchemischen Rechnungen beobachtet wurde. Diese Stabilität könnte im Falle von $n=51 \mathrm{zu}$ einem veränderten Muster der freien OH-Streckschwingung führen. Die hier diskutierten Auffälligkeiten einzelner Clustergrößen liefern Anknüpfungspunkte für den Vergleich mit anderen experimentellen Ansätzen und sollten in Zukunft mit verbesserten Signal-zu-Rausch-Verhältnis reproduziert werden 665, 66]. Insbesondere die OH-Streckschwingung scheint eine empfindliche Sonde für strukturelle Auffälligkeit einzelner Clustergrößen zu sein [27]. 


\section{Zusammenfassung und Ausblick}

\subsection{Zusammenfassung}

Die vorgestellte Arbeit demonstriert die vielseitigen Anwendungen der IR-CIPIMethode, wie die größenselektive Beobachtung der Kristallisation von großen Wasserclustern $(n>100)$ und die Untersuchung der Strukturen kleiner Cluster $(n<100)$. Die Infrarot-anregungsmodulierte, durch chemische Vorionisation verstärkte Photoionisation beruht auf dem Effekt einer Signalverstärkung durch IR-Absorption bei schwellennaher Photoionisation, der von Steinbach und Buck 38, 39 zuerst beschrieben wurde.

Die mit dieser Methode erhaltenen IR-Spektren können für verschiedene Größenbereiche mit durch andere Verfahren generierten Spektren verglichen werden. Alle in dieser Arbeit diskutierten, größenselektiven Verfahren wurden erst in den letzten Jahren entwickelt 26-28, 37]. Für mittlere Clustergrößen ab $n=19$ sind die erhaltenen IR-Spektren sehr ähnlich zu RIDIR-Spektren von phenoldotierten Clustern [37. Dieser Befund zeigt, dass vorwiegend eine Anlagerung des Natriums auf der Oberfläche der Wassercluster erfolgt und ab dieser Größe das Wasserstoffbrückennetzwerk des Clusters kaum beeinflusst wird. Die Arbeit von Fujii und Mizuse hat die Übereinstimmung zwischen phenoldotierten und hydroniumionendotierten Clustern ab einer Größe von $n=49$ demonstriert 27]. Die Spektren natriumdotierter Cluster sind im Bereich $n=113 \pm 12$ für alle verwendeten Expansionsbedingungen sehr ähnlich und zeigen ein amorphes Verhalten. Ebenfalls vergleichbar sind die IR-Spektren amorpher $\mathrm{Na}\left(\mathrm{H}_{2} \mathrm{O}\right)_{225 \pm 25}$-Cluster mit denen ionischer $\mathrm{Na}^{+}\left(\mathrm{H}_{2} \mathrm{O}\right)_{250 \pm 5}$-Cluster aus der Gruppe von Williams [26]. Die Erzeugung der Spektren der ionischen Spezies erfolgte mittels IR(M)PD. Für die größten, 
mit der IR-CIPI Methode untersuchten Cluster der Größe $n=575 \pm 25(d \approx 4 \mathrm{~nm})$ wurden für den Vergleich direkte Absorptionsmessungen von Nanopartikeln in Hüllstromzellen herangezogen [43], die über keine Größenselektion verfügen. Da das in Hüllstromzellen durchgeführte Experiment somit über die Größenverteilung gemittelt ist, gibt dieses die spezifische Absorption von Eis erst bei Partikeln mit einem gemittelten Durchmesser von $8 \mathrm{~nm}$ wieder. Dagegen stimmt das beobachtete Absorptionsmuster der IR-CIPI-Methode gut mit den Vorhersagen von Buch et al. für einen im Kern kristallinen Cluster der Größe $n=600$ und 931 überein [44]. Eine größenselektive Untersuchung der Kristallisation von Wasserclustern wurde für mehrere unterschiedliche Expansionsbedingungen durchgeführt, bei der ein von diesen Expansionsbedingungen abhängiges Einsetzen beobachtet werden konnte. Je höher die Endtemperatur der Cluster ist, desto „früher“ (bei kleineren Größen) kann der Übergang vom amorphen zum kristallinen Cluster stattfinden und desto „eher“ kann die spezifische Absorption des kristallinen Kerns um $3200 \mathrm{~cm}^{-1}$ beobachtet werden. Der größenselektiv gemessene Verlauf der IR-Absorption ist in Übereinstimmung mit den Vorhersagen von Buch et al. [44], allerdings mit dem Unterschied, dass die Oberflächenoszillatoren eine stärkere Absorption zeigen. Eine mögliche Erklärung wäre eine höhere Sensitivität des genutzten Wirkungseffekts auf die DDA-Oszillatoren der Oberfläche. Dies scheint auch für andere größenselektive Verfahren zu gelten [26, 28, 37].

Für die Clustergröße $n=20$ konnte mit Hilfe einer kombiniert experimentellen und quantenchemischen Studie der IR-Absorption im OH-Streckbereich das erste Auftreten eines vierfach koordinierten Wassermoleküls im Inneren eines neutralen Wasserclusters nachgewiesen werden [115. Dieses Merkmal eines Clusters wurde ab der Größe $n=17$ für ungerade Cluster prognostiziert 73, 74. Das experimentelle Spektrum des $\mathrm{Na}\left(\mathrm{H}_{2} \mathrm{O}\right)_{20}$-Clusters kann für den Fall der Expansion mit Neon mit dem simulierten Spektrum aus zwei Strukturen dargestellt werden: Dem tröpfchenartigen Isomer mit innenliegendem Wassermolekül und dem aus zahlreichen quantenchemischen Studien bekannten, über die Kanten verbundenen Prisma 73, 74, 172, 173. In dieser symmetrischen Struktur bewirkt die Anlagerung von Natrium eine Ringöffnung und damit eine Aufspaltung des DAA-Motivs. Findet die Expansion mit Argon statt, kann das gemessene Spektrum allein über das tröpfchenartige Isomer erklärt werden. Über die thermodynamische Stabilität der Isomere kann aufgrund kinetischer Effekte bei der Abkühlung mit Argon oder 
Neon keine Aussage getroffen werden. Die Clustergröße $n=25$ zeigt im Fall von Argon und Neon ein ähnliches Absorptionsmuster und kann mit Hilfe eines einzelnen Isomers beschrieben werden, welches kein internes Wassermolekül aufweist. Somit deutet sich an, dass Cluster ohne internes Wassermolekül bis zu dieser Größe auftreten können. Für die Clustergröße $n=32$ konnten sieben Isomere mit ähnlicher Wasserstoffbrückentopologie und zwei internen Wassermolekülen die Absorption des experimentellen Spektrums exemplarisch wiedergeben.

Die erhaltenen Ergebnisse zeigen die Vielseitigkeit der IR-CIPI Methode. Sie liefert simultan in einer Messung (einem IR-Laserscan) über einen weiten Größenbereich (ca. $n=15-600$ ) die Strukturinformationen für zahlreiche Clustergrößen. Darüber hinaus bietet sie die Möglichkeit, die Kristallisation von Wasserclustern größenselektiv zu untersuchen. Die hier erhaltenen Ergebnisse bieten der Quantenchemie Referenzen für die Weiterentwicklung ihrer Modellpotentiale zur Beschreibung von Wasseraggregaten und ihrer Suchalgorithmen zur Exploration der energetisch günstigsten Strukturen.

\subsection{Ausblick}

Die erhaltenen Ergebnisse können durch weitere Studien ergänzt werden, die im Folgenden vorgestellt werden.

In dieser Arbeit konnte das Einsetzen der Kristallisation größenselektiv bestimmt werden, jedoch ist nicht bekannt, wann im Laufe der Expansion diese auftritt. Eine mögliche Erklärung wäre, dass durch die Kollision mit Natrium die Barriere zwischen amorphem und kristallinem Cluster überschritten wird, da das Zeitfenster von $240 \mu \mathrm{s}$ im Falle des Trägergases Helium ausreichend groß ist. Huang et al. 154 stellten experimentell fest, dass die Kristallisation in einem Cluster mit 4000 bis 6000 Molekülen nach 35 us abgeschlossen ist. Die Simulationen von Gimelshein et al. 178 zeigen, dass eine Temperierung der Cluster kurz hinter der Düsenöffnung abgeschlossen ist, was auf eine Kristallisation in der Düse deuten würde. Ebenfalls in der Düse geschieht die Kristallisation bei Manka et al., jedoch kommen hier andere Expansionstechniken zum Einsatz [149]. Durch die Variation des Natriumdampfdrucks bei gleichbleibenden Expansionsbedingungen würde die Energie bei der Kollision im Falle der Einfachdotierung verändert werden. Dies 
würde im Fall einer höheren Kollisionsenergie zu einer Kristallisation bei kleineren Clustergrößen führen.

Weitere Wassercluster könnten durch die von Hartke und Dieterich [11 genutzte Methode untersucht werden. Der Größenbereich $n=19-30$ scheint hier aufgrund der geringen Störung durch das Natrium und der Separierung der einzelnen Oszillatoren am vielversprechendsten zu sein. Auch die Clustergrößen $n=43,51,53$, 55 und 60/61 zeigen in dem hier vorgestellten Experiment Besonderheiten im Absorptionsverhalten, die durch weitere Messungen bestätigt werden sollten [79,80. In der theoretischen Studie von Kazachenko et al. wurde beispielsweise für $n=51$ mit fünf Modellpotentialen jeweils die gleiche Minimustruktur mit besonders hohen Bindungsenergien pro Wassermolekül gefunden.

Neue Möglichkeiten bezüglich der Signal-zu-Rausch-Verbesserungen bietet das hier vorgestellte UV/Vis-Lasersystem. Der Festkörperlaser ermöglicht eine bessere Synchronisation mit dem IR-Lasersystem, sodass ein Schuss-zu-Schuss-Modus etabliert werden kann. Bei diesem wird das UV/Vis-System mit einer Frequenz von $10 \mathrm{~Hz}$ und das IR-Lasersystem mit $5 \mathrm{~Hz}$ betrieben. Erste Untersuchungen dieses Modus wurden von Sabine Wolff in ihrer Masterarbeit durchgeführt [140]. Weiterhin enthalten ihre Untersuchungen Delaymessungen, bei denen der zeitliche Versatz zwischen den Laserpulsen variiert wird, um aufgrund der nun geringeren Pulsbreite die maximale Verstärkung für das Experiment zu bestimmen. Eventuell haben die unterschiedlichen Oszillatoren von Oberfläche und Kern delayabhängige Maxima, sodass der Wirkungseffekt auf diesem Wege genauer aufgeklärt werden könnte. Jedoch ist aus Messungen von flüssigem Wasser bekannt, dass die Umordnung der Wasserstoffbrückentopologie innerhalb von Picosekunden abläuft [6, 7], was mit dem vorhandenen Experiment auf Clusterebene nicht untersucht werden könnte.

Das neue UV/Vis-Lasersystem ermöglicht die Aufnahme von Photionisationsspektren bis zur Sättigung des Signals, um die IE des Isomers mit angelagertem Natrium zu bestimmen [66]. Weiterhin führt die unterschiedliche Isomerenaktivät zwischen den Neon- und den Argonexpansionen zu dem Schluss, dass unterschiedliche, von der Kühlrate abhängige Ionisationsenergien vorliegen könnten.

Die durch die Expansion erhaltenen kristallinen Nanopartikel könnten mit Hilfe einer Kühlfalle eingefangen werden, um mittels Röntgenbeugungsverfahren die Struktur (amorphe/kristalline Anteile) näher zu bestimmen [65. 


\section{A. Anhang}

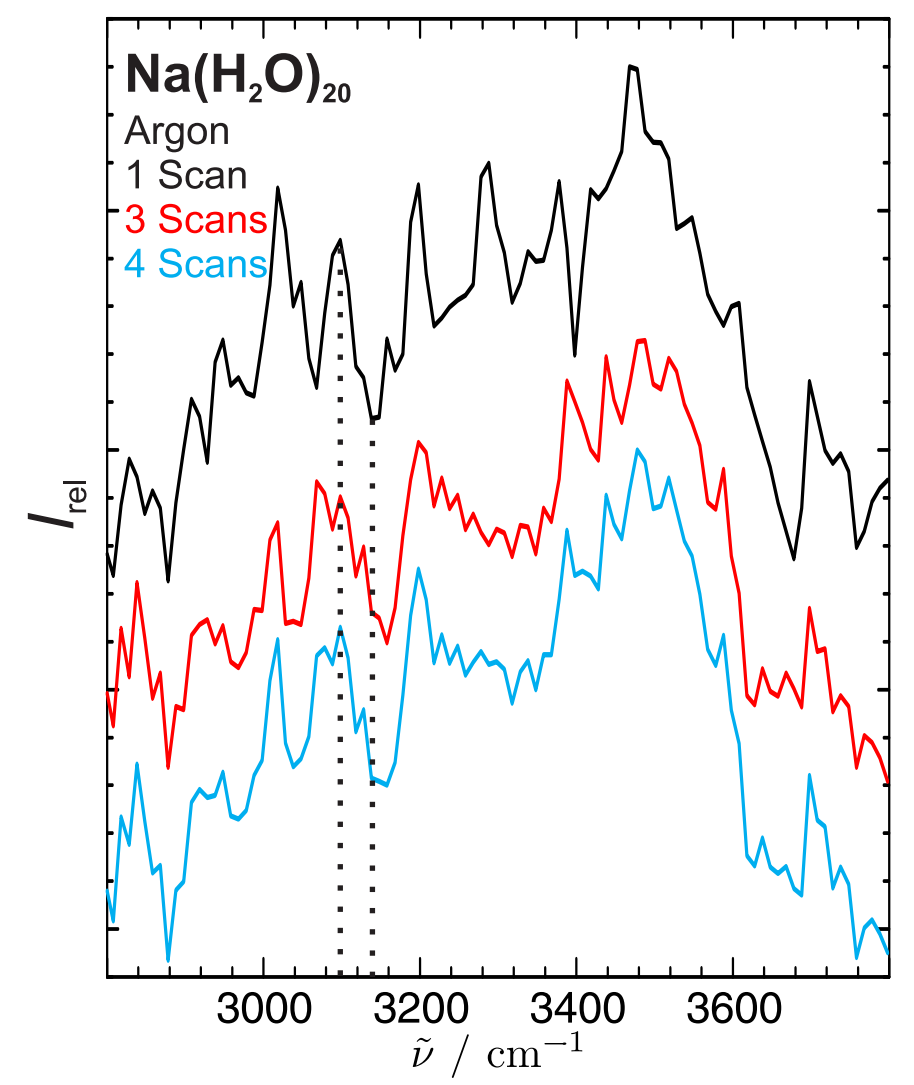

Abbildung A.1.: Veranschaulichung des statistischen Rauschens für die IRSpektren von $\mathrm{Na}\left(\mathrm{H}_{2} \mathrm{O}\right)_{20}$ erzeugt in einer Argonexpansion. Das schwarze Spektrum stellt eine einzelne Messung vom 20.12.2011, die weiteren Spektren stellen Mittelungen mehrere Messtage dar. In rot: 12.12.11, 05.01.12 und 13.01.12, in blau: Eine Mittelung aller Spektren. 


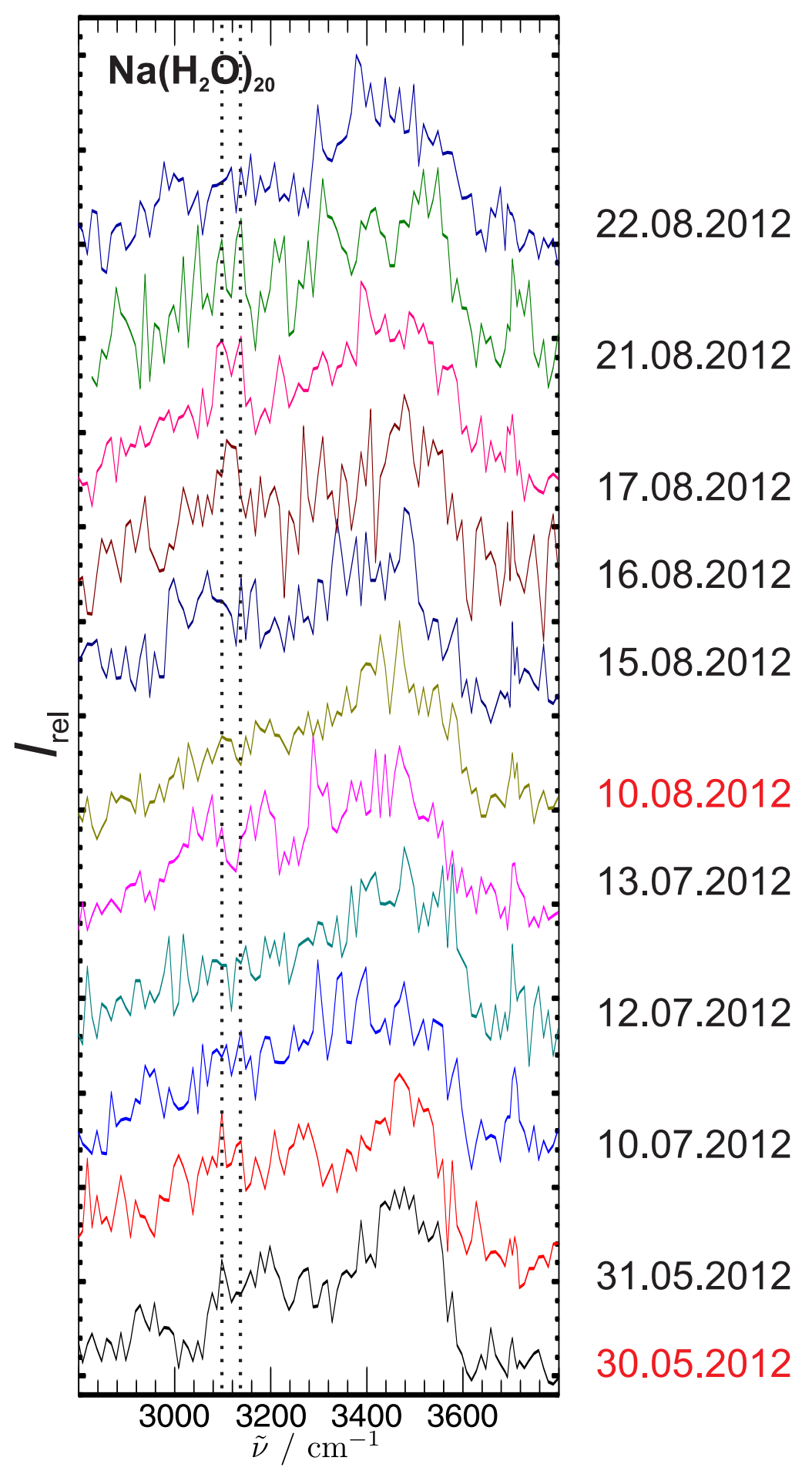

Abbildung A.2.: Die IR-Spektren der einzelnen Messtage von $\mathrm{Na}\left(\mathrm{H}_{2} \mathrm{O}\right)_{20}$ erzeugt in einer Neonexpansion. Die in rot gekennzeichneten Messtage wurden für die Gesamtmittelung nicht herangezogen, da die Absorption auf einen größeren Anteil an Isomer B schließen lässt. 


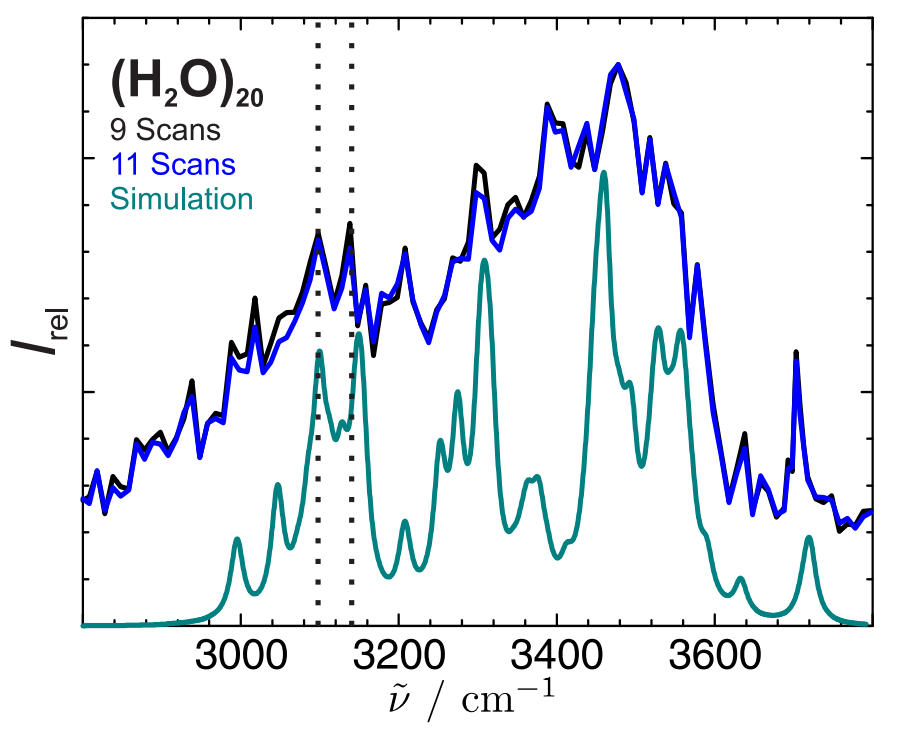

Abbildung A.3.: Vergleich der aus neun und elf Messungen gemittelten IRSpektren von $\mathrm{Na}\left(\mathrm{H}_{2} \mathrm{O}\right)_{20}$ erzeugt in einer Neonexpansion mit der Simulation des IR-Spektrums von $\left(\mathrm{H}_{2} \mathrm{O}\right)_{20}$ m einem Absorbtionsanteil von $65 \%$ Isomer B und 35\% Isomer A. Die Frequenzen sind mit MP2 unter Verwendung des Basissatzes aug-cc-pVDZ berechnet und mit dem Faktor 0,96 skaliert, sowie um $20 \mathrm{~cm}^{-1}$ Lorentz-Verbreitert.

\section{A.1. Verwendete Chemikalien}

Die verwendeten Chemikalien sind alle kommerziell erhältlich und wurden für die Messungen nicht weiter bearbeitet. In Tabelle A.1 wird ein kurzer Überblick über die verwendeten Chemikalien gegeben. Laserfarbstoffe für die Farbtstofflaser werden nicht aufgelistet.

\section{A.2. Publikationen}

Im Rahmen dieser Arbeit sind folgende Publikationen entstanden:

- F. Zurheide, C. W. Dierking, C. C. Pradzynski, R. M. Forck, F. Flüggen, U. Buck und T. Zeuch. A Size Resolved Infrared Spectroscopic Study of Structural Transitions in Sodium Doped $\left(\mathrm{H}_{2} \mathrm{O}\right)_{n}$ Clusters Containing Ten to Hundred Water Molecules, J. Phys. Chem. A, 2015, 119, 2709-2720. 
Tabelle A.1.: Liste der verwendeten Chemikalien.

\begin{tabular}{c|c|c|c|c}
\hline Substanz & Formel & CAS Nr. & Reinheit & Lieferant \\
\hline Natrium & $\mathrm{Na}$ & $7440-23-5$ & unbekannt & unbekannt \\
Wasser (bidestilliert) & $\mathrm{H}_{2} \mathrm{O}$ & $7732-18-5$ & unbekannt & IPC Göttingen \\
Helium & $\mathrm{He}$ & $7440-59-7$ & $\geq 99,996 \%$ & Linde \\
Neon & $\mathrm{Ne}$ & $7440-01-9$ & $\geq 99.99 \%$ & Air Liquide \\
Argon & $\mathrm{Ar}$ & $7440-37-1$ & $\geq 99,998 \%$ & Air Liquide \\
Xenon & $\mathrm{Xe}$ & $7440-63-3$ & $\geq 99,998$ & Messer \\
1,4-Dioxan & $\mathrm{C}_{4} \mathrm{H}_{8} \mathrm{O}_{2}$ & $123-91-1$ & $\geq 99,5 \%$ & Carl Roth \\
Ethanol, vergällt & $\mathrm{C}_{2} \mathrm{H}_{6} \mathrm{O}$ & $64-17-5$ & $\geq 99,8 \%$ & Carl Roth \\
\hline
\end{tabular}

- C. C. Pradzynski, C. W. Dierking, F. Zurheide, R. M. Forck, U. Buck, T. Zeuch und S. S. Xantheas. Infrared detection of $\left(\mathrm{H}_{2} \mathrm{O}\right)_{20}$ isomers of exceptional stability: a drop-like and a face-sharing pentagonal prism cluster, Phys. Chem. Chem. Phys., 2014, 16, 26691-26696.

- U. Buck, C. C. Pradzynski, T. Zeuch, J. M. Dieterich, B. Hartke. A size resolved investigation of large water clusters, Phys. Chem. Chem. Phys., 2014, 16, 6859-6871.

- J. Lengyel, A. Pysanenko, J. Kočišek, V. Poterya, C. C. Pradzynski, T. Zeuch, P. Slavíč und M. Fárník. Nucleation of Mixed Nitric AcidWater Ice Nanoparticles in Molecular Beams that Starts with a $\mathbf{H N O}_{3}$ Molecule, J. Phys. Chem. Lett., 2012, 3, 3096-3101.

- C. C. Pradzynski, R. M. Forck, T. Zeuch, P. Slavíček, U. Buck. A Fully Size-Resolved Perspective on the Crystallization of Water Clusters, Science, 2012, 337, 1529-1532.

- R. M. Forck, J. M. Dieterich, C. C. Pradzynski, A. L. Huchting, R. A. Mata, T. Zeuch. Structural diversity in sodium doped water trimer, Phys. Chem. Chem. Phys., 2012, 14, 9054-9057.

- R. M. Forck, C. C. Pradzynski, S. Wolff, M. Ončák, P. Slavíček und T. Zeuch. Size resolved infrared spectroscopy of $\mathrm{Na}\left(\mathrm{CH}_{3} \mathrm{OH}\right)_{n}(n=4-7)$ clusters in the $\mathrm{OH}$ stretching region: Unravelling the interaction of 
methanol clusters with a sodium atom and the emergence of the solvated electron, Phys. Chem. Chem. Phys., 2012, 9, 3004-3016. 


\section{A.3. Auflistung der Expansionsparameter der verwendeten Messreihen.}

Tabelle A.2.: Auflistung der Expansionsparameter der verwendeten Messreihen. „10er" und ,5er“ bezeichnen die letzte Ziffer der am IR-Laser eingestellten Wellenzahlen.

\begin{tabular}{|c|c|c|c|c|c|c|}
\hline Messtag & Gas & $\frac{p_{\text {exp }}}{\text { bar }}$ & $\frac{\vartheta_{\text {Dise }}}{{ }_{\mathrm{C}}^{\mathrm{C}}}$ & $\frac{\vartheta_{\text {Ofen }}}{{ }^{\mathrm{C}}}$ & $\frac{\lambda_{\text {PI }}}{\mathrm{nm}}$ & $10 \mathrm{er} / 5 \mathrm{er}$ \\
\hline \hline 12.12 .2011 & $\mathrm{Ar}$ & 1.0 & 70 & 55 & 360 & $10 \mathrm{er}$ \\
20.12 .2011 & $\mathrm{Ar}$ & 1.3 & 70 & 50 & 360 & $5 \mathrm{er}$ \\
05.01 .2012 & $\mathrm{Ar}$ & 1.7 & 95 & 85 & 388 & $10 \mathrm{er}$ \\
13.01 .2012 & $\mathrm{Ar}$ & 1.3 & 80 & 70 & 388 & $5 \mathrm{er}$ \\
\hline \hline 02.06 .2010 & $\mathrm{He}$ & 1.5 & 87 & 77 & 400 & $5 \mathrm{er} / 10 \mathrm{er}\left(3330 \mathrm{~cm}^{-1}-3550 \mathrm{~cm}^{-1}\right)$ \\
04.06 .2010 & $\mathrm{He}$ & 1.5 & 87 & 77 & 400 & $5 \mathrm{er} / 10 \mathrm{er}\left(3550 \mathrm{~cm}^{-1}-3800 \mathrm{~cm}^{-1}\right)$ \\
07.06 .2010 & $\mathrm{He}$ & 1.5 & 87 & 77 & 400 & $5 \mathrm{er} / 10 \mathrm{er}\left(3100 \mathrm{~cm}^{-1}-3330 \mathrm{~cm}^{-1}\right)$ \\
08.06 .2010 & $\mathrm{He}$ & 1.5 & 87 & 77 & 400 & $5 \mathrm{er} / 10 \mathrm{er}\left(2900 \mathrm{~cm}^{-1}-3100 \mathrm{~cm}^{-1}\right)$ \\
\hline 06.03 .2012 & $\mathrm{He}$ & 3.5 & 130 & 120 & 390 & $5 \mathrm{er} / 10 \mathrm{er}$ \\
08.03 .2012 & $\mathrm{He}$ & 3.5 & 130 & 120 & 390 & $5 \mathrm{er} / 10 \mathrm{er}$ \\
15.03 .2012 & $\mathrm{He}$ & 3.9 & 135 & 130 & 390 & $5 \mathrm{er} / 10 \mathrm{er}$ \\
21.03 .2012 & $\mathrm{He}$ & 3.9 & 135 & 130 & 390 & $5 \mathrm{er} / 10 \mathrm{er}$ \\
22.03 .2012 & $\mathrm{He}$ & 4.0 & 135 & 130 & 390 & $5 \mathrm{er} / 10 \mathrm{er}$ \\
27.03 .2012 & $\mathrm{He}$ & 4.0 & 135 & 130 & 390 & $5 \mathrm{er} / 10 \mathrm{er}$ \\
11.04 .2012 & $\mathrm{He}$ & 4.5 & 145 & 140 & 390 & $5 \mathrm{er} / 10 \mathrm{er}$ \\
13.04 .2012 & $\mathrm{He}$ & 4.5 & 145 & 140 & 390 & $5 \mathrm{er} / 10 \mathrm{er}$ \\
02.05 .2012 & $\mathrm{He}$ & 3.5 & 130 & 120 & 360 & $5 \mathrm{er} / 10 \mathrm{er}$ \\
04.05 .2012 & $\mathrm{He}$ & 3.5 & 130 & 120 & 360 & $5 \mathrm{er} / 10 \mathrm{er}$ \\
09.05 .2012 & $\mathrm{He}$ & 3.8 & 135 & 130 & 360 & $5 \mathrm{er} / 10 \mathrm{er}$ \\
\hline \hline 30.05 .2012 & $\mathrm{Ne}$ & 2.3 & 160 & 90 & 360 & $10 \mathrm{er}$ \\
31.05 .2012 & $\mathrm{Ne}$ & 2.3 & 110 & 100 & 360 & $10 \mathrm{er}$ \\
10.07 .2012 & $\mathrm{Ne}$ & 2.5 & 110 & 100 & 360 & $10 \mathrm{er}$ \\
12.07 .2012 & $\mathrm{Ne}$ & 2.3 & 160 & 95 & 360 & $10 \mathrm{er}$ \\
13.07 .2012 & $\mathrm{Ne}$ & 2.3 & 110 & 100 & 360 & $10 \mathrm{er}$ \\
10.08 .2012 & $\mathrm{Ne}$ & 2.3 & 110 & 100 & 360 & $10 \mathrm{er}$ \\
15.08 .2012 & $\mathrm{Ne}$ & 2.3 & 110 & 100 & 385 & $10 \mathrm{er}$ \\
16.08 .2012 & $\mathrm{Ne}$ & 2.3 & 110 & 100 & 388 & $10 \mathrm{er}$ \\
17.08 .2012 & $\mathrm{Ne}$ & 2.3 & 160 & 100 & 388 & $10 \mathrm{er}$ \\
21.08 .2012 & $\mathrm{Ne}$ & 3.0 & 110 & 100 & 388 & \\
22.08 .2012 & $\mathrm{Ne}$ & 2.3 & 160 & 100 & 388 & \\
\hline
\end{tabular}




\section{A.4. Matlab-Skripte}

\section{A.4.1. Nach Clustergrößen aufgelöste Auswertung}

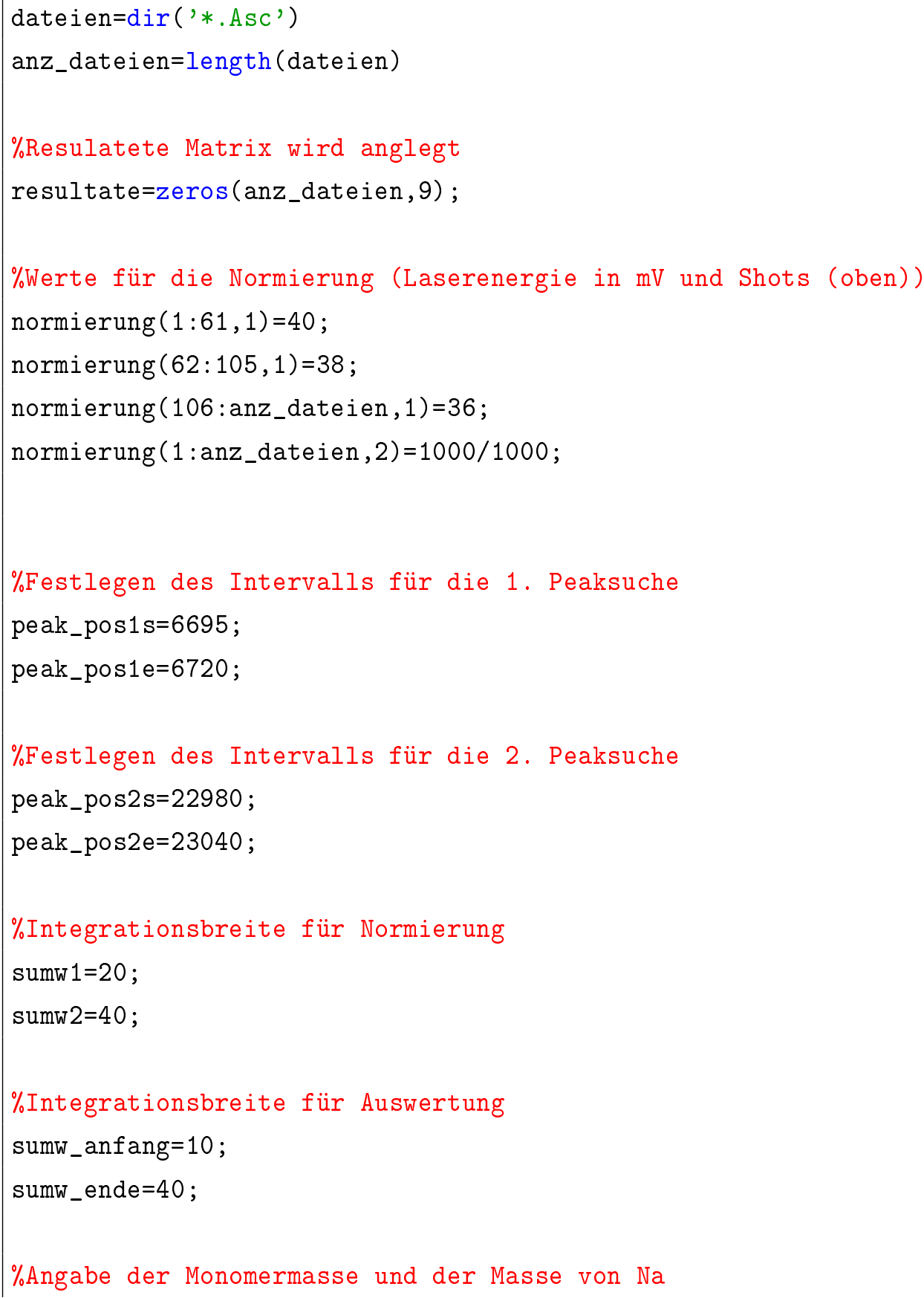




\section{A. Anhang}

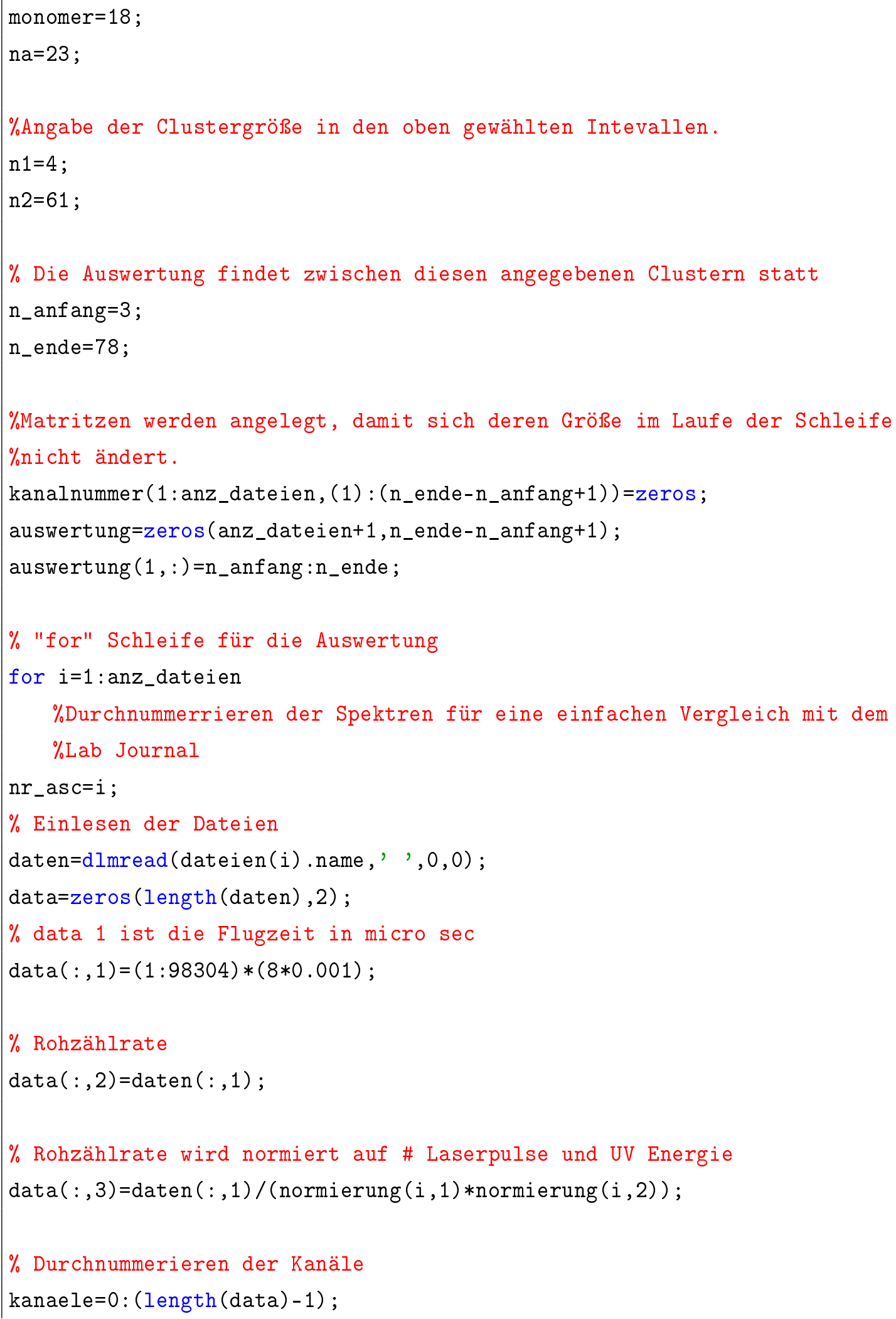




\section{$\%$ Kanalnummern werden angelegt}

$\operatorname{data}(:, 4)=$ kanaele';

$\%$ Nummerierung der Dateien

resultate $(i, 1)=n r_{-}$asc ;

$\%$ Hier wird das Maximum im Intervall [peak_pos1s,peak_pos1e] in der 3.

$\%$ Spalte wiedergegeben

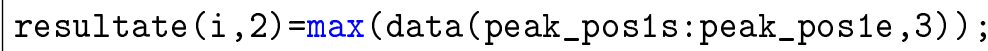

$\%$ Um zu Integrieren muss der Index des Maximums bestimmt werden. Gibt es

$\%$ mehrere Maxima, wird das erste genommen. (. .,1)

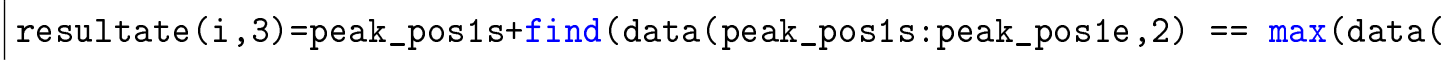
peak_pos1s: peak_pos1e,2)),1);

$\%$ Es wird um das Maximum +- der Breite sumw1 summiert

resultate $(i, 4)=\operatorname{sum}(\operatorname{data}(\operatorname{resultate}(i, 3)-\operatorname{sumw} 1: \operatorname{resultate}(i, 3)+\operatorname{sumw} 1,2))$;

$\%$ Hier wird das Maximum im Intervall [peak_pos2s,peak_pos2e] in der 3.

$\%$ Spalte wiedergegeben

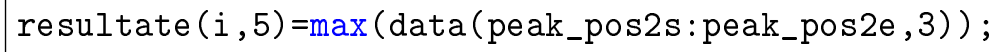

$\%$ Um zu integrieren muss der Index des Maximums bestimmt werden. Gibt es

$\%$ mehrere Maxima, wird das erste genommen. (..,1)

resultate $(i, 6)=$ peak_pos $2 \mathrm{~s}+f$ ind $\left(\right.$ data $_{\text {(peak_pos } 2 \mathrm{~s}: \text { peak_pos } 2 e, 3)==\max (\text { data }}($ peak_pos2s : peak_pos2e,3)),1);

$\%$ Es wird um das Maximum +- der Breite sumw2 summiert

resultate $(i, 7)=\operatorname{sum}(\operatorname{data}(\operatorname{resultate}(i, 3)-\operatorname{sumw} 2: \operatorname{resultate}(i, 3)+\operatorname{sumw} 2,2))$;

$\%$ Bestimmung Eichparameter t_o und $c$

$\mathrm{m} 1=(\mathrm{n} 1 *$ monomer+na $)$;

$\mathrm{m} 2=(\mathrm{n} 2 *$ monomer+na $)$;

resultate $(i, 8)=(m 2 * \operatorname{resultate}(i, 3)-m 1 * \operatorname{resultate}(i, 6)+(\operatorname{resultate}(i, 3)-$ resultate $(i, 6)) * \operatorname{sqrt}(\mathrm{m} 1 * \mathrm{~m} 2)) /(\mathrm{m} 2-\mathrm{m} 1)$;

$\%$ c Berechnung

resultate $(i, 9)=((\operatorname{resultate}(i, 3)-\operatorname{resultate}(i, 8)) \wedge 2) / \mathrm{m} 1$;

100

$101 \%$ Umrechnung in Massendomäne 
for $n=1:$ length (data)

$\operatorname{data}(n, 5)=(\operatorname{data}(n, 4)-\operatorname{resultate}(i, 8)) \wedge 2 / \operatorname{resultate}(i, 9)$;

end

\%Umrechnung in Clustergröße

for $j=1:$ length (data)

$\operatorname{data}(j, 6)=(\operatorname{data}(j, 5)-$ na $) /$ monomer ;

end

$\%$ Anfertigung eines bar-plots, mit dem die Normierung überprüft werden $\%$ kann

$\mathrm{x} \lim \left(\left[\begin{array}{ll}0 & 50\end{array}\right]\right)$

$\%$ Der erste Kanal wird 0 gesetzt, da unphysikalisch. Somit wird eine bessere

\%automatische Y-Achsen Skalierung erreicht

$\operatorname{data}(1,2)=0$

$\operatorname{bar}(\operatorname{data}(:, 6), \operatorname{data}(:, 2))$

\%Integration; in der ersten Spalte soll die Clustergröße stehen, darunter $\%$ die summierten Signale

$\%$ Um zu summieren, wird von ganzzahliger Clustergröße auf Kanalnummern $\%$ zurückgerechnet

$\operatorname{masse}\left((1):\left(n_{-}\right.\right.$ende- $n_{-}$anfang +1$\left.)\right)=\left(\left(n_{\text {_anfang }}: n_{-}\right.\right.$ende $) *$ monomer+na $)$;

kanalnummer $\left(i,(1):\left(n_{-}\right.\right.$ende-n_anfang +1$\left.)\right)=$ round $($ resultate $(i, 8)+\operatorname{sqrt}($ masse $*$ resultate $(i, 9))$ );

$\%$ Es wird mit der linear zunehmender Breite (sumw_ende-sumw_anfang)/( $\mathrm{n}_{-}$ende-n_anfang) um die Cluster summiert und die Ergebnisse in $\%$ eine Matrix 'auswertung' geschrieben

$\%$ zunahme $=\left((\right.$ sumw_ende-sumw_anfang $\left.) /\left(n_{\text {_ende-n_anfang }}\right)\right)$

for $l=n_{-}$anf ang: $n_{-}$ende;

\%auswertung ( $i+1,1-n_{-}$anf ang +1$)=$ sum (data (kanalnummer ( $i, l-n_{-}$anf ang +1$)-(((1-$ $\left.\mathrm{n}_{\text {_anfang }}+1\right) *\left((\right.$ sumw_ende-sumw_anf ang $\left.\left.) /\left(\mathrm{n}_{\text {_ende-n_anfang }}\right)\right)\right):$ kanalnummer (i , $1-n_{-}$anfang +1$)+\left(\left(1-n_{-}\right.\right.$anfang +1$) *\left((\right.$ sumw_ende-sumw_anfang $) /\left(n_{-}\right.$ende- 


$$
\text { n_anfang))) ), 2)) ; }
$$

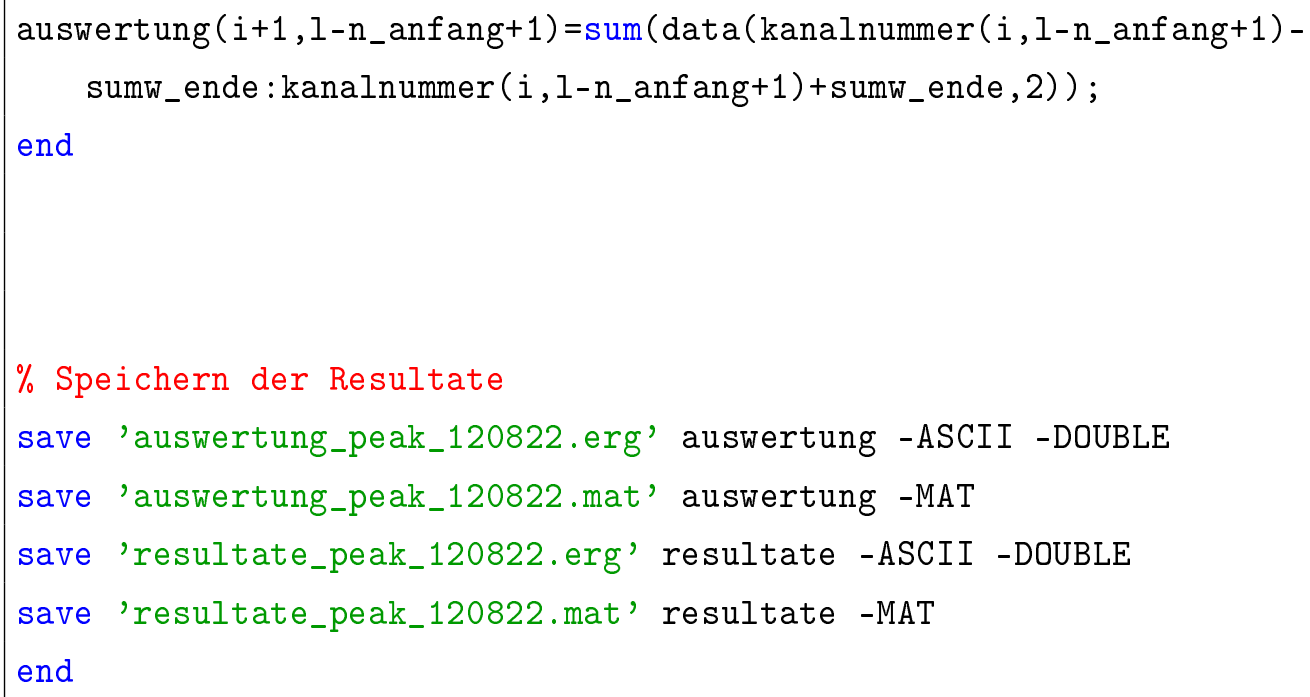

\section{A.4.2. Auswertung nach Größenbereichen}

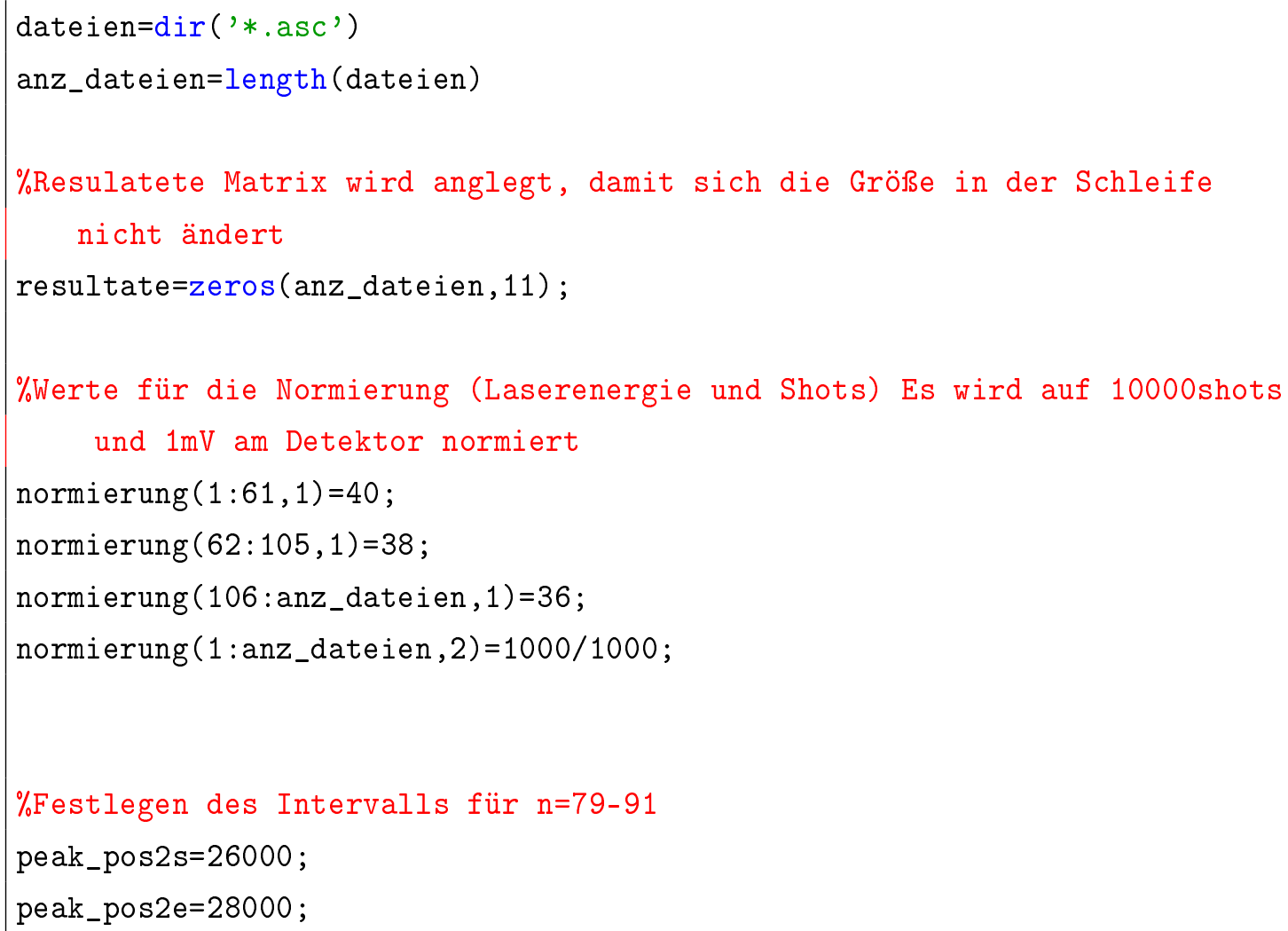




\section{A. Anhang}

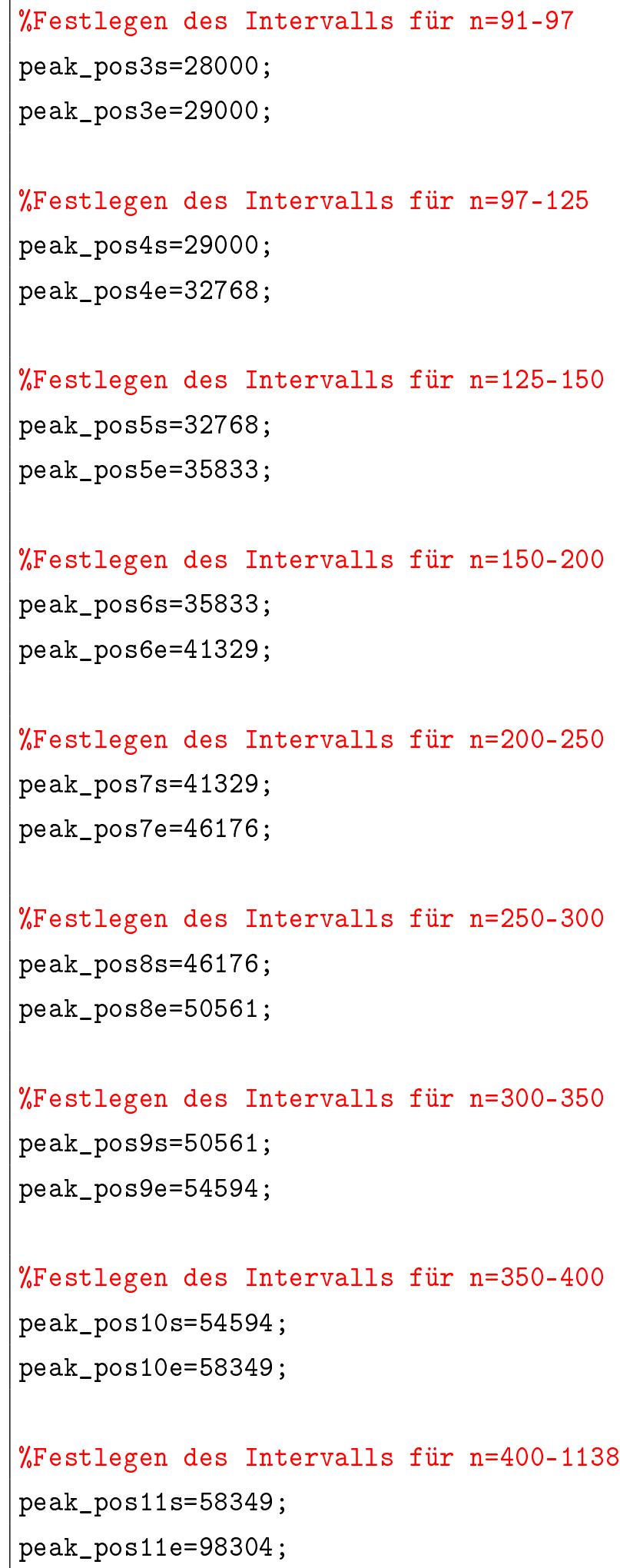




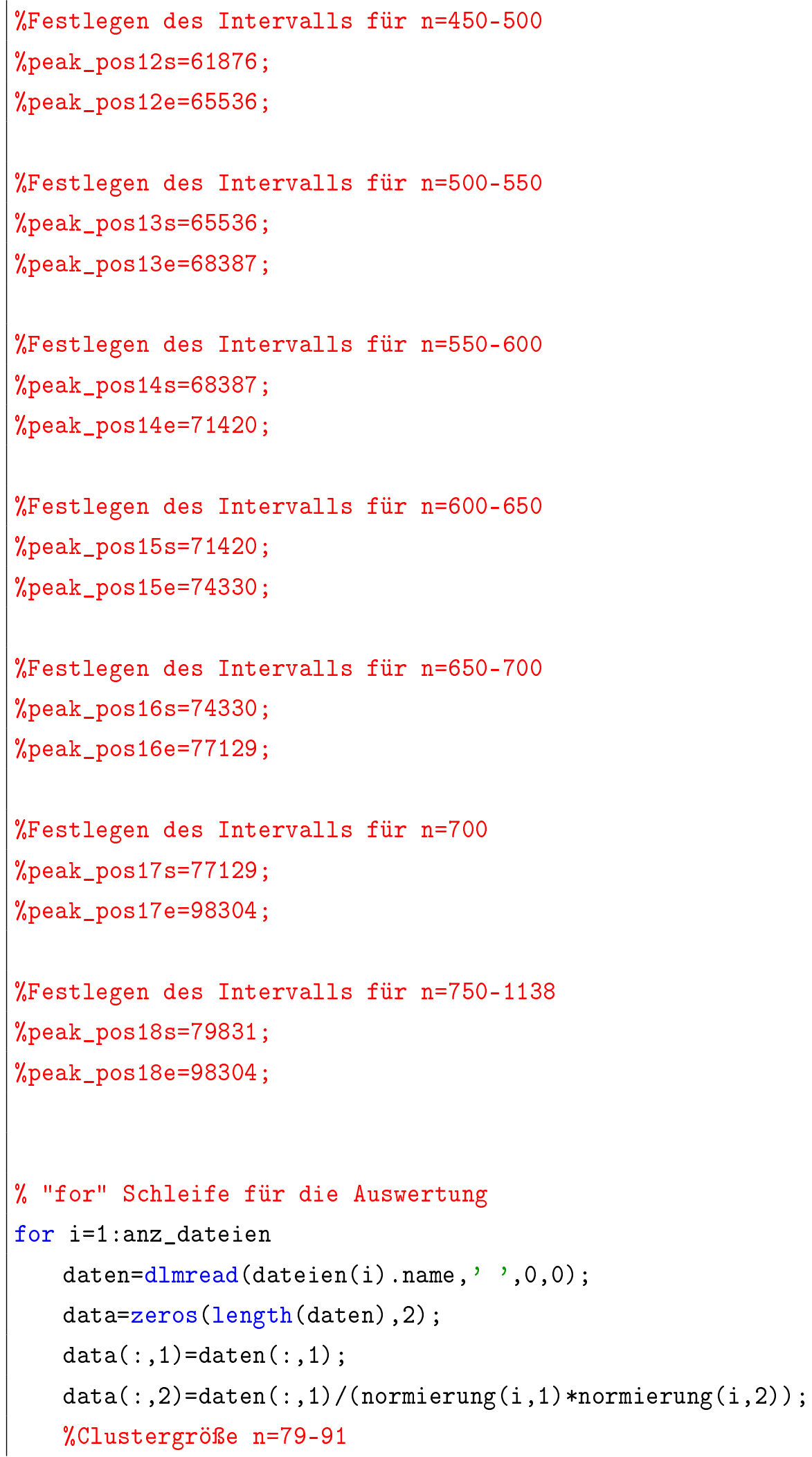




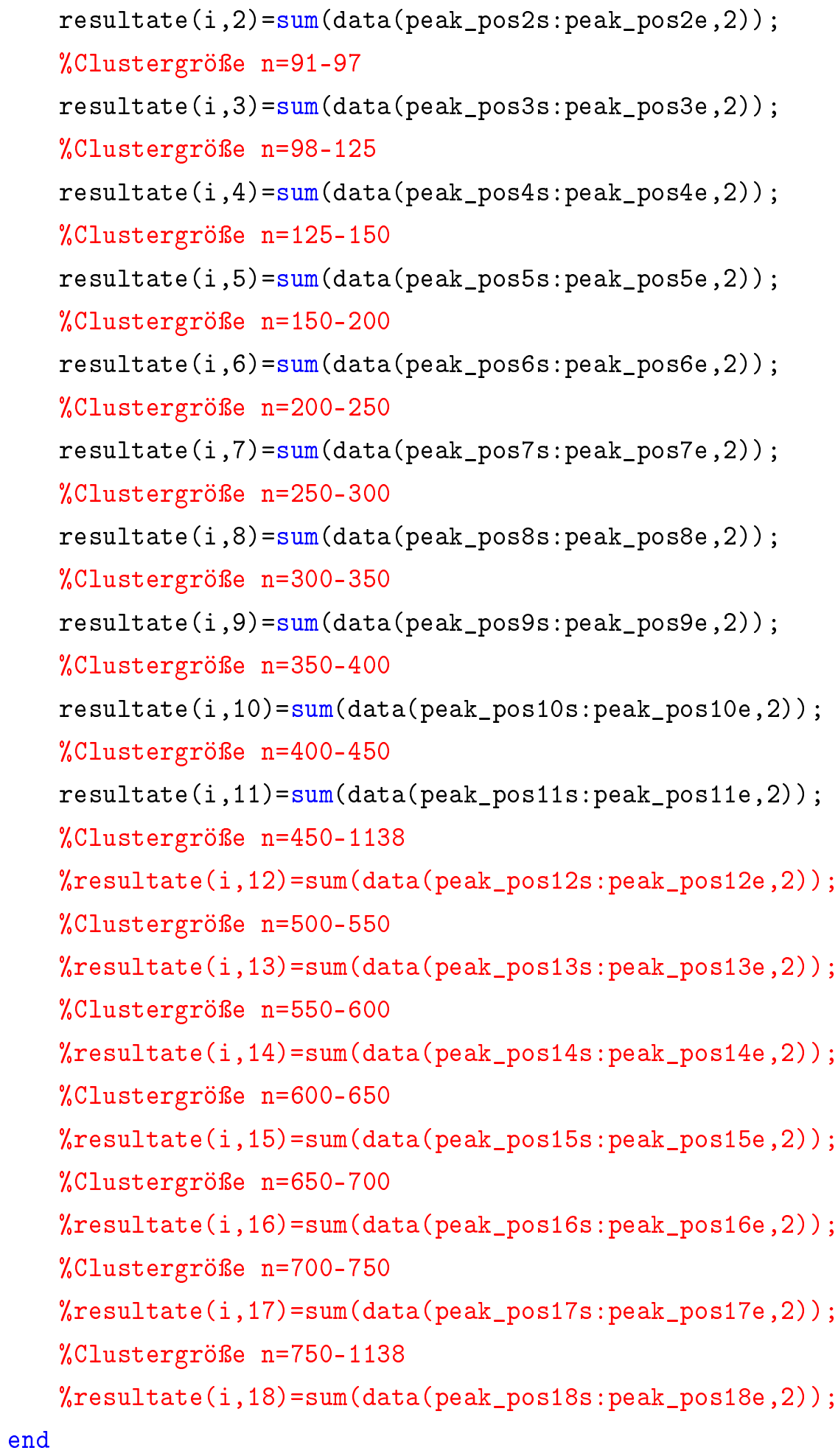




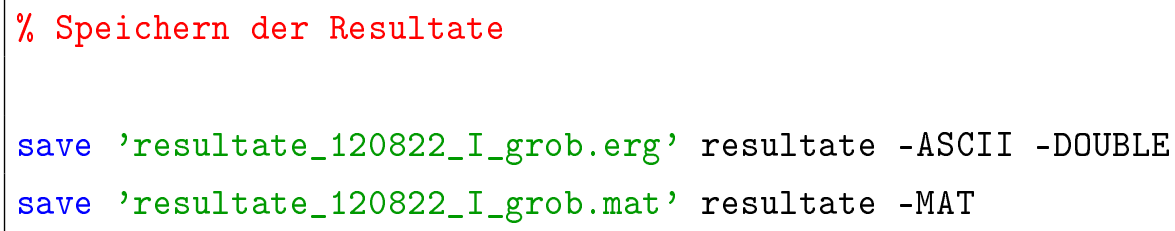





\section{Literaturverzeichnis}

[1] E. M. Knipping, M. J. Lakin, K. L. Foster, P. Jungwirth, D. J. Tobias, R. B. Gerber, D. Dabdub, B. J. Finlayson-Pitts. Experiments and Simulations of Ion-Enhanced Interfacial Chemistry on Aqueous $\mathrm{NaCl}$ Aerosols. Science, 288:301-306, 2000.

[2] M. Kulmala. How Particles Nucleate and Grow. Science, 302:1000-1001, 2003.

[3] Q. Shi, S. D. Belair, J. S. Francisco und S. Kais. On the interactions between atmospheric radicals and cloud droplets: A molecular picture of the interface. Proc. Natl. Acad. Sci., 100(17):9686-9690, 2003.

[4] R. Zhang, A. Khalizov, L. Wang, M. Hu und W. Xu. Nucleation and Growth of Nanoparticles in the Atmosphere. Chem. Rev., 112(3):1957-2011, 2012.

[5] O. Mishima und H. E. Stanley. The relationship between liquid, supercooled and glassy water. Nature, 396:329-335, 1998.

[6] C. P. Lawrence und J. L. Skinner. Vibrational spectroscopy of HOD in liquid $\mathrm{D}_{2} \mathrm{O}$. III. Spectral diffusion, and hydrogen-bonding and rotational dynamics. J. Chem. Phys., 118(1):264-272, 2003.

[7] T. Schäfer, J. Lindner, P. Vöhringer und D. Schwarzer. OD stretch vibrational relaxation of HOD in liquid to supercritical $\mathrm{H}_{2} \mathrm{O}$. J. Chem. Phys., $130(22): 224502,2009$.

[8] M. Fárník und V. Poterya. Atmospheric processes on ice nanoparticles in molecular beams. Frontiers in Chemistry, 2(4):1-9, 2014.

[9] U. Buck und F. Huisken. Infrared spectroscopy of size-selected water and methanol clusters. Chem. Rev., 100:3863-3890, 2000. 
[10] M. A. Suhm und F. Kollipost. Femtisecond single-mole infrared spectroscopy of molecular clusters. Phys. Chem. Chem. Phys., 15:10702-10721, 2013.

[11] U. Buck, C. C. Pradzynski, T. Zeuch, J. M. Dieterich und B. Hartke. A size resolved investigation of large water clusters. Phys. Chem. Chem. Phys., 16:6859-6871, 2014.

[12] L. M. Goss, S. W. Sharpe, T. A. Blake, V. Vaida, und J. W. Brault. Direct Absorption Spectroscopy of Water Clusters. J. Phys. Chem. A, 103:86208624, 1999.

[13] D. J. Nesbitt, T. Häber and M. A. Suhm. General Discussion. Faraday Discuss., 118:305-306, 2001.

[14] A. Moudens, R. Georges, M. Goubet, J. Makarewicz, S. E. Lokshtanov und A. A. Vigasin. Direct absorption spectroscopy of water clusters formed in a continuous slit nozzle expansion. J. Chem. Phys., 131:204312, 2009.

[15] K. E. Otto, Z. Xue, P. Zielke und M. A. Suhm. The Raman spectrum of isolated water clusters. Phys. Chem. Chem. Phys., 16:9849-9858, 2014.

[16] G. G. Brown, B. C. Dian, K. O. Douglass S. M. Geyer,S. T. Shipman und B. H. Pate. A broadband Fourier transform microwave spectrometer based on chirped pulse excitation. Rev. Sci. Instrum., 79(5):053103, 2008.

[17] C. Pérez, M. T. Muckle, D. P. Zaleski, N. A. Seifert, B. Temelso, G. C. Shields, Z. Kisiel und B. H. Pate. Structures of Cage, Prism, and Book Isomers of Water Hexamer from Broadband Rotational Spectroscopy. Science, 336:897-901, 2012.

[18] C. Pérez, S. Lobsiger, N. A. Seifert, D. P. Zaleski, B. Temelso, G. C. Shields, Z. Kisiel und B. H. Pate. Broadband Fourier transform rotational spectroscopy for structure determination: The water heptamer. Chem. Phys. Lett., 571:1 - 15, 2013.

[19] U. Buck und H. Meyer. Scattering analysis of cluster beams: Formation and fragmentation of small $\mathrm{Ar}_{n}$ clusters. Phys. Rev. Lett., 52:109-112, 1984. 
[20] M. Okumura, L. I. Yeh, J. D. Myers and Y. T. Lee. Infrared spectra of the cluster ions $\mathrm{H}_{7} \mathrm{O}_{3}^{+} \cdot \mathrm{H}_{2}$ and $\mathrm{H}_{9} \mathrm{O}_{4}^{+} \cdot \mathrm{H}_{2}$. J. Chem. Phys., 85:2328-2329, 1986.

[21] L. I. Yeh, M. Okumura, J. D. Myers, J. M. Price und Y. T. Lee. Vibrational spectroscopy of the hydrated hydronium cluster ions $\mathrm{H}_{3} \mathrm{O}^{+}\left(\mathrm{H}_{2} \mathrm{O}\right)_{n}(n=1,2$, 3). J. Chem. Phys., 91:7319-7330, 1989.

[22] L. I. Yeh, Y. T. Lee, J. T. Hougen. Vibration-Rotation Spectroscopy of the Hydrated Hydronium Ions $\mathrm{H}_{5} \mathrm{O}_{2}^{+}$and $\mathrm{H}_{9} \mathrm{O}_{4}^{+}$. J. Mol. Spectrosc., 164:473488, 1994.

[23] U. Buck. Structure and Dynamics of Small Size Selected Molecular Clusters. J. Phys. Chem., 98(20):5190-5200, 1994.

[24] U. Buck, I. Ettischer, M. Melzer, V. Buch und J. Sadlej. Structure and Spectra of Three-Dimensional $\left(\mathrm{H}_{2} \mathrm{O}\right)_{n}$ Clusters, $n=8,9$, 10. Phys. Rev. Lett., 80(12):2578-2581, 1998.

[25] K. Mizuse, N. Mikami und A. Fujii. Infrared Spectra and Hydrogen-Bonded Network Structures of Large Protonated Water Clusters $\mathrm{H}^{+}\left(\mathrm{H}_{2} \mathrm{O}\right)_{n}(n=20-$ 200). Angew. Chem., 49:10119-10122, 2010.

[26] J. T. O'Brien and E. R. Williams. Effects of Ions on Hydrogen-Bonding Water Networks in Large Aqueous Nanodrops. J. Am. Chem. Soc., 134(24):10228-10236, 2012.

[27] A. Fujii and K. Mizuse. Infrared spectroscopic studies on hydrogen-bonded water networks in gas phase clusters. Int. Rev. Phys. Chem., 32(2):266-307, 2013 .

[28] J. A. Fournier, C. J. Johnson, C. T. Wolke, G. H. Weddle, A. B. Wolk und M. A. Johnson. Vibrational spectral signature of the proton defect in the three-dimensional $\mathrm{H}^{+}\left(\mathrm{H}_{2} \mathrm{O}\right)_{21}$ cluster. Science, 344:1009-1012, 2014.

[29] K. R. Asmis, G. Santambrogio, J. Zhou, E. Garand, J. Headrick, D. Goebbert, M. A. Johnson und D. M. Neumark. Vibrational spectroscopy of hydrated electron clusters $\mathrm{H}_{2} \mathrm{O}_{15-50}^{-}$via infrared multiple photon dissociation. J. Chem. Phys., 126:191105, 2007. 
[30] N. I. Hammer, J.-W. Shin, J. M. Headrick, E. G. Diken, J. R. Roscioli, G. H. Weddle und M. A. Johnson. How Do Small Water Clusters Bind an Excess Electron? Science, 306:675-679, 2004.

[31] N. I. Hammer, J. R. Roscioli, J. C. Bopp, J. M. Headrick und M. A. Johnson. Vibrational predissociation spectroscopy of the $\left(\mathrm{H}_{2} \mathrm{O}\right)_{(6-21)}^{-}$clusters in the $\mathrm{OH}$ stretching region: Evolution of the excess electron-binding signature into the intermediate cluster size regime. J. Chem. Phys., 123:244311, 2005.

[32] C. Bobbert, S. Schütte, C. Steinbach und U. Buck. Fragmentation and reliable size distributions of large ammonia and water clusters. Eur. Phys. J. D, 19:183-192, 2002.

[33] J. H. Litman, B. L. Yoder, B. Schlappi und R. Signorell. Sodium-doping as a reference to study the influence of intracluster chemistry on the fragmentation of weakly-bound clusters upon vacuum ultraviolet photoionization. Phys. Chem. Chem. Phys., 15:940-949, 2013.

[34] J. Lengyel, A. Pysanenko, V. Poterya, J. Kočišek und M. Fárník. Extensive water cluster fragmentation after low energy electron ionization. Chem. Phys. Lett., 612:256-261, 2014.

[35] Ch. Janzen, D. Spangenberg, W. Roth und K. Kleinermanns. Structure and vibrations of phenol $\left(\mathrm{H}_{2} \mathrm{O}\right)_{7,8}$ studied by infrared-ultraviolet and ultravioletultraviolet double-resonance spectroscopy and ab initio theory. J. Chem. Phys., 110(20):9898-9907, 1999.

[36] T. Hamashima und A. Fujii K. Mizuse. Infrared Spectroscopy of Phenol$\left(\mathrm{H}_{2} \mathrm{O}\right)_{n>10}$ : Structural Strains in Hydrogen Bond Networks of Neutral Water Clusters. J. Phys. Chem. A, 113(44):12134-12141, 2009.

[37] T. Hamashima, K. Mizuse und A. Fujii. Spectral signatures of fourcoordinated sites in water clusters: Infrared spectroscopy of phenol- $\left(\mathrm{H}_{2} \mathrm{O}\right)_{n}$ $(\sim 20 \leq n \leq \sim 50)$. J. Phys. Chem. A, 115:620-625, 2011.

[38] C. Steinbach und U. Buck. Vibrational Spectroscopy of Size-Selected Sodium-Doped Water Clusters. J. Phys. Chem. A, 110:3128-3131, 2006. 
[39] U. Buck, I. Dauster, B. Gao und Z. Liu. Infrared spectroscopy of small sodium-doped water clusters: Interaction with the solvated electron. J. Phys. Chem. A, 111:12355-12362, 2007.

[40] R. M. Forck, J. M. Dieterich, C. C. Pradzynski, A. L. Huchting, R. A. Mata und T. Zeuch. Structural diversity in sodium doped water trimers. Phys. Chem. Chem. Phys., 14:9054-9057, 2012.

[41] R. Forck. Strukturaufklärung von Natrium-Solvens-Clustern durch IRanregungsmodulierte Photoionisationsspektroskopie, 2012. Dissertation, Georg-August-Universität Göttingen.

[42] R. M. Forck, C. C. Pradzynski, S. Wolff, M. Ončák, P. Slavíček und T. Zeuch. Size resolved infrared spectroscopy of $\mathrm{Na}\left(\mathrm{CH}_{3} \mathrm{OH}\right)_{n} \quad(n=4-7)$ clusters in the $\mathrm{OH}$ stretching region: unravelling the interaction of methanol clusters with a sodium atom and the emergence of the solvated electron. Phys. Chem. Chem. Phys., 14:3004-3016, 2012.

[43] C. Medcraft, D. McNaughton, C. D. Thompson, D. R. T. Appadoo, S. Bauerecker und E. G. Robertson. Water ice nanoparticles: size and temperature effects on the mid-infrared spectrum. Phys. Chem. Chem. Phys., 15:36303639, 2013.

[44] V. Buch, S. Bauerecker, J. P. Devlin, U. Buck und J. K. Kazimirski. Solid water clusters in the size range of tens-thousands of $\mathrm{H}_{2} \mathrm{O}$ : a combined computational/spectroscopic outlook. J. Chem. Phys., 23(3):375-433, 2004.

[45] G. Torchet, P. Schwartz, J. Farges, M. F. de Feraudy und B. Raoult. Structure of solid water clusters formed in a free jet expansion. J. Chem. Phys., 79(12):6196-6202, 1983.

[46] I. Dauster, M. A. Suhm, U. Buck und T. Zeuch. Experimental and theoretical study of the microsolvation of sodium atoms in methanol clusters: Differences and similarities to sodium/water and sodium/ammonia. Phys. Chem. Chem. Phys., 10:83-95, 2008. 
[47] C. C. Pradzynski, R. M. Forck, T. Zeuch, P. Slavíček und U. Buck. A Fully Size-Resolved Perspective on the Crystallization of Water Clusters. Science, 337:1529-1532, 2012.

[48] H. Haberland. Clusters of Atoms and Molecules I - Theory, Experiment and Clusters of Atoms. Berlin: Springer-Verlag, 1994.

[49] H. Haberland. Clusters of Atoms and Molecules II - Solvation and Chemistry of Free Clusters, and Embedded, Supported and Compressed Clusters. Berlin: Springer-Verlag, 1994.

[50] G. Scoles, editor. Atomic and Molecular Beam Methods, Volume 1. Oxford University Press, 1988.

[51] G. Scoles, editor. Atomic and Molecular Beam Methods, Volume 2. Oxford University Press, 1988.

[52] O. F. Hagena. Nucleation and growth of clusters in expanding nozzle flows. Surf. Sci., 106:101-116, 1981.

[53] O. F. Hagena. Cluster ion sources (invited). Rev. Sci. Instrum., 63(4):23742379, 1992.

[54] J. M. Soler, N. García, O. Echt, K. Sattler und E. Recknagel. Microcluster growth: Transition from successive monomer addition to coagulation. Phys. Rev. Lett., 49:1857-1860, 1982.

[55] O. Knospe, R. Schmidt und G. Seifert. Percolation approach for atomic and molecular cluster formation. Physics Letters A, 129(4):236-240, 1988.

[56] O. Knospe, R. Schmidt und J. Knoll. First stages of matter growth in a dynamical percolation model. Physics Letters A, 147(1):23-27, 1990.

[57] H. Chen, R.-b. Huang, X. Lu, Z.-C. Tang, X. Xu und L.-S. Zheng. Studies on carbon/sulfur cluster anions produced by laser vaporization: Experiment (collision-induced dissociation) and theory (ab initio calculation). I. $\mathrm{C}_{2} \mathrm{~S}_{m}^{-}$ $(1 \leq m \leq 11)$. J. Chem. Phys., 112(21):9310-9318, 2000. 
[58] E. Arunan, G. R. Desiraju, R. A. Klein, J. Sadlej, S. Scheiner, I. Alkorta, D. C. Clary, R. H. Crabtree, J. J. Dannenberg, P. Hobza, H. G. Kjaergaard, A. C. Legon, B. Mennucci und D. J. Nesbitt. Definition of the hydrogen bond (IUPAC Recommendations 2011). Pure Appl. Chem., 83(8):1637-1641, 2011.

[59] J. J. Dannenberg, L. Haskamp, A. Masunov. Are Hydrogen Bonds Covalent Or Electrostatic? A Molecular Orbital Comparison of Molecules in Electric Fields and H-Bonding Environments. J.Phys. Chem. A, 103:7083-7086, 1999.

[60] R. M. Forck, I. Dauster, Y. Schieweck, T. Zeuch, U. Buck, M. Ončák und P. Slavíček. Communications: Observation of two classes of isomers of hydrated electrons in sodium-water clusters. J. Chem. Phys., 132:221102, 2010.

[61] R. M. Forck, I. Dauster, U. Buck und T. Zeuch. Sodium microsolvation in ethanol: common features of $\mathrm{Na}(\mathrm{HO}-\mathrm{R})_{n}\left(\mathrm{R}=\mathrm{H}, \mathrm{CH}_{3}, \mathrm{C}_{2} \mathrm{H}_{5}\right)$ clusters. J. Phys. Chem. A, 115:6068-6076, 2011.

[62] C. P. Schulz, R. Haugstätter, H. U. Tittes und I. V. Hertel. Free sodiumwater clusters: photoionisation studies in a pulsed molecular beam source. Z. Phys. D, 10(1767):279-290, 1988.

[63] I. V. Hertel, C. Hüglin, C. Nitsch und C. P. Schulz. Photoionization of $\mathrm{Na}\left(\mathrm{NH}_{3}\right)_{n}$ and $\mathrm{Na}\left(\mathrm{H}_{2} \mathrm{O}\right)_{n}$ Clusters: A Step Towards the Liquid Phase? Phys. Rev. Lett., 67(13):1767-1770, 1991.

[64] T. Zeuch und U. Buck. Sodium doped hydrogen bonded clusters: Solvated electrons and size selection. Chem. Phys. Lett., 579:1-10, 2013.

[65] F. Zurheide, vsl. 2016. Dissertation, Georg-August-Universität Göttingen.

[66] C. W. Dierking, vsl. 2017. Dissertation, Georg-August-Universität Göttingen.

[67] W. Kern und M. Karplus. The water molecule. In Felix Franks, editor, Water - A Comprehensive Treatise, Volume I. Plenum Press, 1972. 
[68] K. Liu, M. G. Brown und R. J. Saykally. Terahertz Laser Vibration-Rotation Tunneling Spectroscopy and Dipole Moment of a Cage Form of the Water Hexamer. J. Phys. Chem. A, 101:8995-9010, 1997.

[69] C. Steinbach, P. Andersson, M. Melzer, J. K. Kazimirski, U. Buck und V. Buch. Detection of the book isomer from the OH-stretch spectroscopy of size selected water hexamers. Phys. Chem. Chem. Phys., 6:3320-3324, 2004.

[70] C. J. Tainter und J. L. Skinner. The water hexamer: Three-body interactions, structures, energetics, and $\mathrm{OH}$-stretch spectroscopy at finite temperature. J. Chem. Phys., 137:104304, 2012.

[71] J. Brudermann, M. Melzer, U. Buck, J. K. Kazimirski, J. Sadlej und V. Buch. The asymmetric cage structure of $\left(\mathrm{H}_{2} \mathrm{O}\right)_{7}$ from a combined spectroscopic and computational study. J. Chem. Phys., 110(22):10649-10652, 1999.

[72] B. Bandow und B. Hartke. Larger Water Clusters with Edges and Corners on Their Way to Ice: Structural Trends Elucidated with an Improved Parallel Evolutionary Algorithm. J. Phys. Chem. A, 110(17):5809-5822, 2006.

[73] B. Hartke. Size-dependent transition from all-surface to interior-molecule structures in pure neutral water clustersy. Phys. Chem. Chem. Phys., 5:275$284,2003$.

[74] A. Lagutschenkov, G. S. Fanourgakis, G. Niedner-Schatteburg und Sotiris S. Xantheas. The spectroscopic signature of the "all-surface" to "internally solvated" structural transition in water clusters in the $n=17-21$ size regime. J. Chem. Phys., 122(19):194310, 2005.

[75] S. Kazachenko und A. J. Thakkar. Water nanodroplets: Predictions of five model potentials. J. Chem. Phys., 138:194302, 2013.

[76] H. Liu, Y. Wang und J. M.Bowman. Quantum Calculations of Intramolecular IR Spectra of Ice Models Using Ab Initio Potential and Dipole Moment Surfaces. J. Phys. Chem. Lett., 3:3671-3676, 2012.

[77] J. K. Kazimirski und V. Buch. Search for Low Energy Structures of Water Clusters $\left(\mathrm{H}_{2} \mathrm{O}\right)_{n}, n=20-22,48,123$, and 293. J. Phys. Chem. A, 107(46):9762-9775, 2003. 
[78] C. C. Pradzynski. Größenselektive Infrarotspektroskopie an Natriumdotierten Wasser-Clustern, 2010. Masterarbeit, Georg-August-Universität Göttingen.

[79] F. Zurheide. Größenselektive Untersuchung natriumdotierter Wasserclustern mittels IR-anregungsmodelierter Photoionisationsspektroskopie, 2013. Diplomarbeit, Georg-August-Universität Göttingen.

[80] C. W. Dierking. Untersuchung der Strukturentwicklung von Wasserclustern zwischen 15 und 100 Molekülen mittels größenselektiver IR-Spektroskopie, 2013. Masterarbeit, Georg-August-Universität Göttingen.

[81] C. G. Salzmann, P. G. Radaelli, E. Mayer und J. L. Finney. Ice XV: A New Thermodynamically Stable Phase of Ice. Phys. Rev. Lett., 103:105701, 2009.

[82] A. Falenty, T. C. Hansen und W. F. Kuhs. Formation and properties of ice XVI obtained by emptying a type sII clathrate hydrate. Nature, 516:231$233,2014$.

[83] J. E. Bertie und E. Whalley. Infrared Spectra of Ices Ih and Ic in the Range 4000 to $350 \mathrm{~cm}^{-1}$. J. Chem. Phys., 40(6):1637-1645, 1964.

[84] T. Loerting, K. Winkel, M. Seidl, M. Bauer, C. Mitterdorfer, P. H. Handle, C. G. Salzmann, E. Mayer, J. L. Finney und D. T. Bowron. How many amorphous ices are there? Phys. Chem. Chem. Phys., 13(10):8783-8794, 2011.

[85] D. T. Bowron, J. L. Finney, A. Hallbrucker, I. Kohl, T. Loerting, E. Mayer und A. K. Soper. The local and intermediate range structures of the five amorphous ices at $80 \mathrm{~K}$ and ambient pressure: A Faber-Ziman and BhatiaThornton analysis. J. Chem. Phys., 125:194502, 2006.

[86] V. Molinero und E. B. Moore. Water Modeled As an Intermediate Element between Carbon and Silicon. J. Phys. Chem. A, 113(13):4008-4016, 2009.

[87] G. Bullock und V. Molinero. Low-density liquid water is the mother of ice: on the relation between mesostructure, thermodynamics and ice crystallization in solutions. Faraday Discuss., 167:371-388, 2013. 
[88] M. S. Elsaesser, K. Winkel, E. Mayer und T. Loerting. Reversibility and isotope effect of the calorimetric glass $\rightarrow$ liquid transition of low-density amorphous ice. Phys. Chem. Chem. Phys., 12:708-712, 2010.

[89] E. B. Moore und V. Molinero. Ice crystallization in water's "no-man's land". J. Chem. Phys., 132(24):244504, 2010.

[90] M. Schmidt und B. von Issendorff. Gas-phase calorimetry of protonated water clusters. J. Chem. Phys., 136:164307, 2012.

[91] C. Hock, M. Schmidt, R. Kuhnen, C. Bartels, L. Ma, H. Haberland und B. von Issendorff. Calorimetric Observation of the Melting of Free Water Nanoparticles at Cryogenic Temperatures. Phys. Rev. Lett., 103:043401, 2009.

[92] J. H. Gross. Mass Spectrometry. Springer, 2004.

[93] H. Budzikiewicz. Massenspektrometrie - Eine Einführung. 4.Auflage, Weinheim: WILEY-VCH, 1998.

[94] A. G. Marshall, C. L. Hendrickson und G. S. Jackson. Fourier transform ion cyclotron resonance mass spectrometry: a primer. Mass Spectrom. Rev., 17(1):1-35, 1998.

[95] L. Ma, K. Majer, F. Chirot und B. von Issendorff. Low temperature photoelectron spectra of water cluster anions. J. Chem. Phys., 131(14):144303, 2009.

[96] J. B. Fenn, M. Mann, C. K. Meng, S. F. Wong, C. M. Whitehouse. Electrospray Ionization for Mass Spectrometry of Large Biomolecules. Science, 246:64-71, 1989.

[97] J. B. Fenn, M. Mann, C. K. Meng, S. F. Wong, C. M. Whitehouse. Electrospray ionization-principles and practice. Mass Spectrom. Rev., 9:37-70, 1990.

[98] M. Karas, D. Bachmann, U. Bahr und F. Hillenkamp. Matrix-assisted ultraviolet laser desorption of non-volatile compounds. Int. J. Mass Spectrom., 78:53-68, 1987. 
[99] M. Karas und F. Hillenkamp. Laser Desorption Ionization of Proteins with Molecular Masses Exceeding 10000 Daltons. Anal. Chem., 60(20):2299-2301, 1988.

[100] W. M. Haynes (Hrsg.). GRC Handbook of Chemistry and Physics, 94th Edition. GRC Press, 2013.

[101] R. M. Garnica, M. F. Appel, L. Eagan, J. R. McKeachie und Th. Benter. A REMPI Method for the Ultrasensitive Detection of $\mathrm{NO}$ and $\mathrm{NO}_{2}$ Using Atmospheric Pressure Laser Ionization Mass Spectrometry. Anal. Chem., 72:5639-5646, 2000.

[102] C. E. H. Dessent und K. Müller-Dethlefs. Hydrogen-Bonding and van der Waals Complexes Studied by ZEKE and REMPI Spectroscopy. Chem. Rev., 100:3999-4021, 2000.

[103] C. J. Gruenloh, J. R. Carney, C. A. Arrington, T. S. Zwier, S. Y. Fredericks, K. D. Jordan. Infrared Spectrum of a Molecular Ice Cube: The $\mathrm{S}_{4}$ and $\mathrm{D}_{2 d}$ Water Octamers in Benzene-(Water) 8 . Science, 276:1678-1681, 1997.

[104] W. A. Alexander, J. P. Wiens, T. K. Minton und G. M. Nathanson. Reactions of solvated electrons initiated by sodium atom ionization at the vacuum-liquid interface. Science, 335:1072-1075, 2012.

[105] J. P. Wiens, G. M. Nathanson, W. A. Alexander, T. K. Minton, S. Lakshmi und G. C. Schatz. Collisions of Sodium Atoms with Liquid Glycerol: Insights into Solvation and Ionization. J. Am. Chem. Soc., 136(8):3065-3074, 2014.

[106] J. Günster, V. Kempter und R. Souda. Sodium Interacting with Amorphous Water Films at 10 and 100 K. J. Phys. Chem. B, 109(36):17169-17173, 2005.

[107] J.-H. Kim, Y.-K. Kim und H. Kang. Hydrolysis of Sodium Atoms on WaterIce Films. Characterization of Reaction Products and Interfacial Distribution of Sodium and Hydroxide Ions. J. Phys. Chem. C, 113(1):321-327, 2009 .

[108] I. Dauster. Die Ionisationsdymanik in Natrium-Solvens-Clustern, 2008. Dissertation, Georg-August-Universität Göttingen. 
[109] W. C. Wiley und I. H. McLaren. Time-of-Flight Mass Spectrometer with Improved Resolution. Rev. Sci. Instrum., 26(12):1150-1157, 1955.

[110] B. A. Mamyrin, V. I. Karataev und D. V. Shmikk. New method for focussing ion bunches in time-of-flight mass spectrometer with high resolution. Sov. Phys. Tech. Phys., 16:1177, 1972.

[111] B. A. Mamyrin, V. I. Karataev, D. V. Shmikk und V. A. Zagulin. The mass-reflectron, a new nonmagnetic time-of-flight mass spectrometer with high solution. Sov. Phys., 37(1):45-48, 1973.

[112] D. Manura und D. Dahl. SIMION (R) 8.0 User Manual, Scientific Instrument Services, Inc. Ringoes, NJ 08551, 2008.

[113] F. Jensen. Computational Chemistry. WILEY-VCH, 1999.

[114] M. J. Frisch, G. W. Trucks, H. B. Schlegel, G. E. Scuseria, M. A. Robb, J. R. Cheeseman, G. Scalmani, V. Barone, B. Mennucci, G. A. Petersson, H. Nakatsuji, M. Caricato, X. Li, H. P. Hratchian, A. F. Izmaylov, J. Bloino, G. Zheng, J. L. Sonnenberg, M. Hada, M. Ehara, K. Toyota, R. Fukuda, J. Hasegawa, M. Ishida, T. Nakajima, Y. Honda, O. Kitao, H. Nakai, T. Vreven, J. A. Montgomery, Jr., J. E. Peralta, F. Ogliaro, M. Bearpark, J. J. Heyd, E. Brothers, K. N. Kudin, V. N. Staroverov, R. Kobayashi, J. Normand, K. Raghavachari, A. Rendell, J. C. Burant, S. S. Iyengar, J. Tomasi, M. Cossi, N. Rega, J. M. Millam, M. Klene, J. E. Knox, J. B. Cross, V. Bakken, C. Adamo, J. Jaramillo, R. Gomperts, R. E. Stratmann, O. Yazyev, A. J. Austin, R. Cammi, C. Pomelli, J. W. Ochterski, R. L. Martin, K. Morokuma, V. G. Zakrzewski, G. A. Voth, P. Salvador, J. J. Dannenberg, S. Dapprich, A. D. Daniels, Ö. Farkas, J. B. Foresman, J. V. Ortiz, J. Cioslowski und D. J. Fox. Gaussian 09 Revision A.02. Gaussian Inc. Wallingford CT 2009.

[115] C. C. Pradzynski, C. W. Dierking, F. Zurheide, R. M. Forck, U. Buck, T. Zeuch und S. S. Xantheas. Infrared detection of $\left(\mathrm{H}_{2} \mathrm{O}\right)_{20}$ isomers of exceptional stability: a drop-like and a face-sharing pentagonal prism cluster. Phys. Chem. Chem. Phys., 16:26691-26696, 2014. 
[116] W. L. Jorgensen, J. Chandrasekhar, J. D. Madura, R. W. Impey und M. L. Klein. Comparison of simple potential functions for simulating liquid water. J. Chem. Phys., 79(2):926-935, 1983.

[117] C. J. Burnham und S. S. Xantheas. Development of transferable interaction models for water. III. Reparametrization of an all-atom polarizable rigid model (TTM2-R) from first principles. J. Chem. Phys., 116(4):1500-1510, 2002.

[118] C. J. Burnham und S. S. Xantheas. Development of transferable interaction models for water. IV. A flexible, all-atom polarizable potential (TTM2-F) based on geometry dependent charges derived from an ab initio monomer dipole moment surface. J. Chem. Phys., 116(12):5115-5124, 2002.

[119] G. S. Fanourgakis und S. S. Xantheas. The Flexible, Polarizable, TholeType Interaction Potential for Water (TTM2-F) Revisited. J. Phys. Chem. A, 110(11):4100-4106, 2006.

[120] G. S. Fanourgakis und S. S. Xantheas. Development of transferable interaction potentials for water. V. Extension of the flexible, polarizable, Thole-type model potential (TTM3-F, v. 3.0) to describe the vibrational spectra of water clusters and liquid water. J. Chem. Phys., 128(7):074506, 2008.

[121] M. Hobein. Photodissoziation und Photoionisation von Molekülclustern, 1994. Dissertation, Georg-August-Universität Göttingen.

[122] S. Schütte. Mehrphotonen- und Elektronenstoßprozesse in Clustern, 1996. Dissertation, Georg-August-Universität Göttingen.

[123] S. Schütte und U. Buck. Ionization potentials of large sodium doped ammonia clusters. Int. J. Mass Spectrosc., 122:183-192, 2005.

[124] C. Steinbach. Streuung und Spektroskopie von Wasserstoffbrückensystemen, 2000. Dissertation, Georg-August-Universität Göttingen.

[125] S. Wolff. Größenselektive Infrarotspektroskopie an Natrium-dotierten Methanol-Clustern, 2011. Bachelorarbeit, Georg-August-Universität Göttingen. 
[126] H. Dehling und B. Haupt. Einführung in die Wahrscheinlichkeitstheorie und Statistik. Springer, 2004.

[127] J. Lengyel, J. Kočišek, V. Poterya, A. Pysanenko, P. Svrčková, M. Fárník, D. K. Zauris und J. Fedor. Uptake of atmospheric molecules by ice nanoparticles: Pickup cross sections. J. Chem. Phys., 137(3):034304, 2012.

[128] W. H. Rodebush und T. DeVries. The vapor pressure of sodium. J. Am. Chem. Soc., 47(10):2488-2493, 1925.

[129] T. E. Gough, M. Mengel, P. A. Rowntree und G. Scoles. Infrared spectroscopy at the surface of clusters: $\mathrm{SF}_{6}$ on Ar. J. Chem. Phys., 83(10):4958-4961, 1985.

[130] U. Buck und H. Pauly. Dampfdruckmessungen an Alkalimetallen. Z. Phys. Chem. N. F., 44:345-352, 1965.

[131] A. Risch. Entwurf eines Tandem-Flugzeit-Massenspektrometers und Messungen der Photodissoziation von Argonclusterionen, 1991. Dissertation, Universität Freiburg.

[132] Wiza, J. L. Microchannel plate detectors. Nuclear Instruments and Methods, 162:587-601, 1979.

[133] Continuum. Powerlite DLS Laser System, Manual, 2010.

[134] W. Koechner und M. Bass. Solid-State Lasers. Springer, 2003.

[135] Continuum. Panther EX OPO \& EX PLUS OPO - Operation and Maintenance, Manual, 2011.

[136] Sirah. Pulsed dye laser, User Manual, 2012.

[137] Continuum. Powerlite Precision II Scientific Laser System, Manual, 2002.

[138] D. Guyer. LaserVison Manual. LaserVision, 2009.

[139] FAST ComTec GmbH. Pr887 User Manual, Version 3.03. 250 ps / $4 \mathrm{GHz}$ Time-of-Flight / Multiscaler, 2009. Benutzer-Handbuch. 
[140] S. Wolff. Analyse der Massenspektren von zeitverzögerter zweistufiger Photoionisation natriumdotierter Wassercluster, 2014. Masterarbeit, GeorgAugust-Universität Göttingen.

[141] T. Watanabe, T. Ebata, S. Tanabe und N. Mikami. Size-selected vibrational spectra of phenol- $\left(\mathrm{H}_{2} \mathrm{O}\right)_{n}(n=1-4)$ clusters observed by IR-UV double resonance and stimulated Raman-UV double resonance spectroscopies. J. Chem. Phys., 105(2):408-419, 1996.

[142] H. Watanabe und S. Iwata. Theoretical studies of geometric structures of phenol-water clusters and their infrared absorption spectra in the $\mathrm{O}-\mathrm{H}$ stretching region. J. Chem. Phys., 105(2):420-431, 1996.

[143] W. Roth, M. Schmitt, Ch. Jacoby, D. Spangenberg, Ch. Janzen und K. Kleinermanns. Double resonance spectroscopy of phenol $\left(\mathrm{H}_{2} \mathrm{O}\right)_{1-12}$ : evidence for ice-like structures in aromate-water clusters? Chem. Phys., 239:1-9, 1998.

[144] E. S. Kryachko und H. Nakatsuji. Ab initio study of lower energy phenolwater $_{1 \leq n \leq 4}$ complexes: Interpretation of two distinct infrared patterns in spectra of phenol-water tetramer. J. Phys. Chem. A, 106(5):731-742, 2002.

[145] T. Ebata, A. Fujii und N. Mikami. Vibrational spectroscopy of small-sized hydrogen-bonded clusters and ther ions. Int. Rev. Phys. Chem., 17(3):331361, 1998.

[146] H. A. Schwarz. Gas phase infrared spectra of oxonium hydrate ions from 2 to 5 1. J. Chem. Phys., 67(12):5525-5534, 1977.

[147] N. I. Hammer, J. R. Roscioli und M. A. Johnson. Identification of Two Distinct Electron Binding Motifs in the Anionic Water Clusters: A Vibrational Spectroscopic Study of the $\left(\mathrm{H}_{2} \mathrm{O}\right)_{6}$ Isomers. J. Phys. Chem. A, 109:78967901, 2005.

[148] M. F. Bush, J. T. O’Brien, J. S. Prell, R. J. Saykally und E. R. Williams. Infrared Spectroscopy of Cationized Arginine in the Gas Phase: Direct Evidence for the Transition from Nonzwitterionic to Zwitterionic Structure. J. Am. Chem. Soc., 129(6):1612-1622, 2007. 
[149] A. Manka, H. Pathak, S. Tanimura, J. Wölk, R. Streya und B. E. Wyslouzil. Freezing water in no-man's land. Phys. Chem. Chem. Phys., 14:4505-4516, 2012.

[150] J. Lengyel, A. Pysanenko, V. Poterya, P. Slavíček, M. Fárník, J. Kočišek und J. Fedor. Irregular shapes of water clusters generated in supersonic expansions. Phys. Rev. Lett., 112:113401, 2014.

[151] J. Sadlej, V. Buch, J. K. Kazimirski und U. Buck. Theoretical Study of Structure and Spectra of Cage Clusters $\left(\mathrm{H}_{2} \mathrm{O}\right)_{n}, n=7-10$. J. Phys. Chem. A, 103(25):4933-4947, 1999.

[152] G. E. Ewing, M. Foster, W. Cantrell und V. Sadtchenko. Thin film water on insulator surfaces. In V. Buch und J. P. Devlin, editor, Water in Confining Geometries, Springer Series in Cluster Physics. S. 179-211. Springer Berlin Heidelberg, 2003.

[153] G. D. Stein und J. A. Armstrong. Structure of water and carbon dioxide clusters formed via homogeneous nucleation in nozzle beams. J. Chem. Phys., 58(5):1999-2003, 1973.

[154] J. Huang und L. S. Bartell. Kinetics of homogeneous nucleation in the freezing of large water clusters. J. Phys. Chem., 99(12):3924-3931, 1995.

[155] L. S. Bartell und Y. G. Chushak. Nucleation of ice in large water clusters: Experiment and simulation. In Victoria Buch and J.Paul Devlin, editors, Water in Confining Geometries, Springer Series in Cluster Physics. S. 399424. Springer Berlin Heidelberg, 2003.

[156] B. J. Murray, D. A. Knopf und A. K. Bertram. The formation of cubic ice under conditions relevant to Earth's atmosphere. Nature, 434:202-205, 2005 .

[157] B. J. Murray und A. K. Bertram. Formation and stability of cubic ice in water droplets. Phys. Chem. Chem. Phys., 8:186-192, 2006.

[158] V. Buch, P. Sandler und J. Sadlej. Simulations of $\mathrm{H}_{2} \mathrm{O}$ Solid, Liquid, and Clusters, with an Emphasis on Ferroelectric Ordering Transition in Hexagonal Ice. J. Phys. Chem. B, 102(44):8641-8653, 1998. 
[159] V. Buch. Treatment of rigid bodies by diffusion Monte Carlo: Application to the para- $\mathrm{H}_{2} \cdots \mathrm{H}_{2} \mathrm{O}$ and ortho- $\mathrm{H}_{2} \cdots \mathrm{H}_{2} \mathrm{O}$ clusters. J. Chem. Phys., 97(1):726-729, 1992.

[160] J. P. Devlin und V. Buch. Ice Nanoparticles and Ice Adsorbate Interactions: FTIR Spectroscopy and Computer Simulations. In V. Buch und J. P. Devlin, editor, Water in Confining Geometries, Springer Series in Cluster Physics. S.425-462. Springer Berlin Heidelberg, 2003.

[161] S. Weng D. M. Wieliczka and M. R. Querry. Wedge shaped cell for highly absorbent liquids: infrared optical constants of water. Appl. Opt., 28(9):17141719, 1989.

[162] A. Y. Zasetsky, A. F. Khalizov, M. E. Earle und J. J. Sloan. Frequency Dependent Complex Refractive Indices of Supercooled Liquid Water and Ice Determined from Aerosol Extinction Spectra. J. Phys. Chem. A, 109(12):2760-2764, 2011.

[163] C. E. Klots. Temperatures of evaporating clusters. Nature, 327:222-223, 1987.

[164] R. S. Berry und D. J. Wales. Freezing, Melting, Spinodals, and Clusters. Phys. Rev. Lett., 63(11):1156-1159, 1989.

[165] C. Hock, C. Bartels, S. Straßburg, M. Schmidt, H. Haberland, B. von Issendorff und A. Aguado. Premelting and postmelting in clusters. Phys. Rev. Lett., 102:043401, 2009.

[166] N. Pugliano und R. J. Saykally. Measurement of Quantum Tunneling Between Chiral Isomers of the Cyclic Water Trimer. Science, 257:1937-1940, 1992.

[167] R. N. Pribble und T. S. Zwier. Size-specific infrared spectra of benzene$\left(\mathrm{H}_{2} \mathrm{O}\right)_{n}$ clusters $(n=1$ through 7$)$ : Evidence for noncyclic $\left(\mathrm{H}_{2} \mathrm{O}\right)_{n}$ structures. Science, 265:75-79, 1994.

[168] K. Nauta und R. E. Miller. Formation of Cyclic Water Hexamer in Liquid Helium: The Smallest Piece of Ice. Science, 287:293-295, 2000. 
[169] J. D. Cruzan, L. B. Braly, Kun Liu, M. G. Brown, J. G. Loeser, und R. J. Saykally. Quantifying Hydrogen Bond Cooperativity in Water: VRT Spectroscopy of the Water Tetramer. Science, 271:59-61, 1996.

[170] J.-W. Shin, N. I. Hammer, E. G. Diken, M. A. Johnson, R. S. Walters, T. D. Jaeger, M. A. Duncan, R. A. Christie und K. D. Jordan. Infrared Signature of Structures Associated with the $\mathrm{H}^{+}\left(\mathrm{H}_{2} \mathrm{O}\right)_{n}(n=6$ to 27$)$ Clusters. Science, 304:1137-1140, 2004.

[171] K. R. Asmis und D. M. Neumark. Vibrational Spectroscopy of Microhydrated Conjugate Base Anions. Acc. Chem. Res., 45(1):43-52, 2012.

[172] G. S. Fanourgakis, E. Aprà, W. A. de Jong und S. S. Xantheas. High-level ab initio calculations for the four low-lying families of minima of $\left(\mathrm{H}_{2} \mathrm{O}\right)_{20}$. II. Spectroscopic signatures of the dodecahedron, fused cubes, face-sharing pentagonal prisms, and edge-sharing pentagonal prisms hydrogen bonding networks. J. Chem. Phys., 135(10):134304-1-134304-9, 2005.

[173] S. S. Xantheas. Low-Lying Energy Isomers and Global Minima of Aqueous Nanoclusters: Structures and Spectroscopic Features of the Pentagonal Dodecahedron $\left(\mathrm{H}_{2} \mathrm{O}\right)_{20}$ and $\left(\mathrm{H}_{3} \mathrm{O}\right)^{+}\left(\mathrm{H}_{2} \mathrm{O}\right)_{20}$. Can. J. Chem. Eng., 90:843-851, 2012.

[174] J. D. Bernal und R. H. Fowler. A Theory of Water and Ionic Solution, with Particular Reference to Hydrogen and Hydroxyl Ions. J. Chem. Phys., 1(8):515-548, 1933.

[175] G. S. Fanourgakis, E. Aprà und S. S. Xantheas. High-level ab initio calculations for the four low-lying families of minima of $\left(\mathrm{H}_{2} \mathrm{O}\right)_{20}$. I. Estimates of MP2/CBS binding energies and comparison with empirical potentials. J. Chem. Phys., 121(6):2655-2663, 2004.

[176] J. P. Furtado, A. P. Rahalkar, S. Shanker, P. Bandyopadhyay und S. R. Gadre. Facilitating Minima Search for Large Water Clusters at the MP2 Level via Molecular Tailoring. J. Phys. Chem. Lett., 3(16):2253-2258, 2012. 
[177] R. M. Young, M. A. Yandell, S. B. King und D. M. Neumark. Thermal effects on energetics and dynamics in water cluster anions $\left(\mathrm{H}_{2} \mathrm{O}\right)_{n}^{-}$. J. Chem. Phys., 136(9):094304, 2012.

[178] N. Gimelshein und S. Gimelshein. persönliche Mitteilung, 2012.

[179] J. M. Dieterich und B. Hartke. OGOLEM: Global cluster structure optimisation for arbitrary mixtures of flexible molecules. A multiscaling, objectoriented approach. Mol. Phys., 108(3-4):279-291, 2010.

[180] E. G. Diken, W. H. Robertson und M. A. Johnson. The Vibrational Spectrum of the Neutral $\left(\mathrm{H}_{2} \mathrm{O}\right)_{6}$ Precursor to the "Magic" $\left(\mathrm{H}_{2} \mathrm{O}\right)_{6}^{-}$Cluster Anion by Argon-Mediated, Population-Modulated Electron Attachment Spectroscopy. J. Phys. Chem. A, 108(1):64-68, 2004.

[181] N. Sahu, S. R. Gadre, A. Rakshit, P. Bandyopadhyay, E. Miliordos und S. S. Xantheas. Low energy isomers of $\left(\mathrm{H}_{2} \mathrm{O}\right)_{25}$ from a hierarchical method based on Monte Carlo temperature basin paving and molecular tailoring approaches benchmarked by MP2 calculations. J. Chem. Phys., 141(16):164304, 2014.

[182] F. Zurheide, C. W. Dierking, C. C. Pradzynski, R. M. Forck, F. Flüggen, U. Buck und T. Zeuch. A Size Resolved Infrared Spectroscopic Study of Structural Transitions in Sodium Doped $\left(\mathrm{H}_{2} \mathrm{O}\right)_{n}$ Clusters Containing Ten to Hundred Water Molecules. J. Phys. Chem. A, 2015, DOI: 10.1021/jp509883m.

[183] S.-W. Lee, P. Freivogel, T. Schindler und J. L Beauchamp. FreezeDried Biomolecules: FT-ICR Studies of the Specific Solvation of Functional Groups and Clathrate Formation Observed by the Slow Evaporation of Water from Hydrated Peptides and Model Compounds in the Gas Phase. J. Am. Chem. Soc., 120(45):11758-11765, 1998. 